\title{
Enhancing Magnetic Resonance Imaging with Computational Fluid Dynamics.
}

Giacomo ANNIO

A dissertation submitted in fulfillment

of the requirements for the degree of

Doctor of Philosophy

of

University College London.

Department of Medical Physics and Biomedical Engineering University College London

September 23, 2020 
I, Giacomo ANNIO, confirm that the work presented in this thesis is my own. Where information has been derived from other sources, I confirm that this has been indicated in the work. 
A Mamma, Papá e Nicola 


\section{Abstract}

Quantitative assessment of haemodynamics has been utilised for better understanding of cardiac function and assisting diagnostics of cardiovascular diseases. To study haemodynamics, magnetic resonance imaging (MRI) and computational fluid dynamics (CFD) are widely used because of their non-invasive nature. It has been demonstrated that the two approaches are complementary to each other with their own advantages and limitations. Four dimensional cardiovascular magnetic resonance (4D Flow CMR) imaging enables direct measurement of blood flow velocity in vivo while spatial and temporal resolutions as well as region of image acquisition are limited to achieve a detailed assessment of the haemodynamics. CFD, on the other hand, is a powerful tool that has the potential to expand the image-obtained velocity fields with some problem-specific assumptions such as rigid arterial walls. We suggest a novel approach in which 4D Flow CMR and CFD are integrated synergistically in order to obtain an enhanced 4D Flow CMRI (EMRI). The enhancement will consist in overcoming the spatial-resolution limitations of the original 4D Flow CMRI, which will enable more accurate quantification of flow dependent bio-mechanical quantities (e.g. endothelial shear stress) as well as non-invasive estimation of blood pressure. At the same time, it will reduce a number of assumptions in conventional haemodynamic CFD such as in/outflow conditions including the effect of valves, the impact of patient-specific vessel wall motion and the effect of the surrounding tissues.

The approach was first tested on a $2 \mathrm{D}$ portion of a pipe, to understand the behaviour of the parameters of the model in this novel framework. Afterwards the methodology was tested on patient specific data, to apply it to the analysis of blood 
flow in a patient specific human aorta, in 2D. The outcomes of EMRI are assessed by comparing the computed velocities with the 4D Flow CMR one.

A fundamental step to allow the translation to clinics of this methodology was the validation. The study was performed on an idealised-simplified model of the human aortic arch - a U bend - with a sinusoidal inflow applied by a pump. Firstly, phase resolved particle image velocimetry (PIV) (an experimental technique enables high spatial-temporal resolution) was performed in 5 different time points of the pump cycle, using a blood alike fluid with the same refractive index matched of the clear silicon phantom, and seeded with silver coated hollow glass spheres. Real time 4D Flow CMR was then performed on the phantom with MRI. Lastly using the pump flow rate and the phantom geometry, a computation of the flow through the $\mathrm{U}$ bend was conducted using Ansys CFX. The flow patterns obtained from the 3 methods were compared in the middle plane of the phantom.

The methodology was then applied to study a patient specific aorta in $3 \mathrm{D}$, and retrieve flow patterns and flow dependent parameters. Finally, the validated methodology was applied to study atherogenesis, and in particular to investigate the relation between EMRI retrieved flow quantities (e.g. wall shear stress (WSS)) and temperature heterogeneity. A carotid artery phantom was realised and studied with CFD, MRT and EMRI.

All the results demonstrate that EMRI preserves flow structures while correcting for experimental noise. Therefore it can provide better insights of the haemodynamics of cardiovascular problems, overcoming the limitations of 4D Flow CMR and CFD, even when considering a small region of interest. These findings were supported by the validation experiment that showed how EMRI retrieved flow patterns were much more consistent with the one measured with high resolution PIV, compensating for 4D Flow CMR errors.

These findings lead to the application to the atherogenesis problem, allowing higher resolution flow patterns, more suitable to be compared to the temperature distribution and highlighted how flow patterns exert an influence on the temperature 
distribution on the vessel wall.

EMRI confirmed its potential to provide more accurate non-invasive estimation of flow derived and flow dependent quantities and become a novel diagnostic tool. 


\section{Impact statement}

The research described in this thesis deals with the implementation of a computational framework that integrates CFD and 4D Flow CMR imaging, the enhanced magnetic resonance imaging (EMRI). In particular this thesis explores the proof of concept of EMRI, its validation and the application to patient specific data and to study of novel biomarkers for atherosclerosis.

This investigation deepens his roots in the need for an accurate description of blood flow in vessels, at the basis of cardiovascular system functioning and cardiovascular diseases. The approach suggested copes with the intrinsic limitations of 4D Flow CMR and CFD, that taken alone leads to an approximate velocity field estimation. On the contrary, EMRI proves to give access to high resolution and fluid dynamic sounds flow fields, which in turn improves the retrieval of important biomechanical markers for cardiovascular diseases (CVDs), such as wall shear stress (WSS) and pressure.

The accuracy of EMRI flow computation was confirmed by the in vitro validation. The development of the experimental setup leads to a novel methodology to generate phantom for flow studies, published in the Journal of Engineering and Science in Medical Diagnostics and Therapy (JESMDT) by American Society of Mechanical Engineering [1]. The same manufacturing methodology was applied to an inter-departmental project leading to another publication [2]. The validation study has been presented to several conferences ( 7 th International Conference on Computational Bioengineering, 8th World Congress of Biomechanics) and a paper has been submitted to the journal IEEE Transaction on Medical Imaging.

The application of EMRI to a patient specific aorta was carried out, showing 
agreement with physiological parameters and the advantage over standard CFD and 4D Flow CMR. This research was presented in a conference (Frontiers of Simulation and Experimentation for Personalised Cardiovascular Management and Treatment), where it was selected within the best computational works and granted an invitation to write a research paper [3]. A work comprising the validation and the application of EMRI has been accepted for an oral presentation to the Virtual Physiological Human 2020 conference.

During the last year of $\mathrm{PhD}$ the fellow established an international collaboration with the Laboratory for Vascular Translational Science (LVTS) of INSERM in Paris, and earned an Erasmus scholarship to fund a three months collaboration. Part of the work carried out during this collaboration is part of this thesis. It deals with the application of EMRI to retrieve flow dependent parameters, and in particular wall shear stress and temperature distribution in athero-prone vessels. This work set the basis for a Marie Curie Fellowship application that was submitted in September 2019.

The suggested computational framework could be potentially applied to the study of CVDs, especially where correct estimate of flow parameters plays a decision making role. To make this translation happen, work needs to be done to extend EMRI to time resolved analysis, to have access to high resolution flow through the cardiac cycle, and to embed this technology in a software package to be of easy use for clinicians. 


\section{Acknowledgements}

I would like to thank my supervisors Gaetano Burriesci, Ryo Torii and Andrea Ducci for the constant support and their patience. I am also grateful to my clinical supervisors Victor Tsang and Vivek Muthurangu and all the staff of Great Ormond Street Hospital for Children, for making clinical facilities and data available.

I would like to thank all the colleagues with which we had an amazing time, funny lunches and nice nights out. Further thanks are due to the research group at LVTS Hopital Bichat for the amazing time I spent in Paris, working on part of this work. In particular I thank Dr. Gregory Franck and Prof. Ralph Sinkus, for their enthusiasm and inspirational thoughts.

I also owe thanks to the EPSRC and CDT in Medical Imaging for funding my project and make all this possible. A further thanks goes to Erasmus Plus to fund the project carried out at LVTS.

Finally I want to thank my family for its never ending support and love. 


\section{Scientific disseminations}

The research carried out during the $\mathrm{PhD}$ and described in this thesis resulted in the following scientific disseminations.

\section{Journal publications}

- Annio, G., Franzetti, G., Bonfanti, M., Gallarello, A., Palombi, A., De Momi, E., ... Torii, R. (2020). 'Low-Cost Fabrication of Polyvinyl Alcohol-Based Personalized Vascular Phantoms for In Vitro Hemodynamic Studies: Three Applications.' Journal of Engineering and Science in Medical Diagnostics and Therapy, 3(3).

- Gallarello, A., Palombi, A., Annio, G., Homer-Vanniasinkam, S., De Momi, E., Maritati, G., ... Wurdemann, H. A. (2019). Patient-Specific Aortic Phantom With Tunable Compliance. Journal of Engineering and Science in Medical Diagnostics and Therapy, 2(4).

- Annio, G., Torii, R., Ariff, B., O’Regan, D. P., Muthurangu, V., Ducci, A., ... Burriesci, G. (2019). 'Enhancing Magnetic Resonance Imaging With Computational Fluid Dynamics.' Journal of Engineering and Science in Medical Diagnostics and Therapy, 2(4).

\section{Conference presentation and posters}

- Annio G., Ducci A., Muthurangu V., Tsang V., Burriesci G., Torii R. (2018). 'Enhanced Magnetic Resonance Imaging' Image-Guided Therapies UK Network+ 06 March 2019, London, UK (Poster presentation) 
- Annio G., Ducci A., Muthurangu V., Tsang V., Burriesci G., Torii R. (2018). 'Enhanced Magnetic Resonance Imaging' VPH-CaSE conference, 19-20 July 2018, London, UK (Oral presentation).

- Annio G., Ducci A., Muthurangu V., Tsang V., Burriesci G., Torii R. (2018). 'Blood flow triple-imaging' 8th World Congress of Biomechanics, 8-12 July 2018, Dublin, Ireland (Poster presentation).

- G Annio, A Ducci, V Muthurangu, V Tsang, G Burriesci, R Torii. (2017), 'Blood flow triple imaging' 7th International Conference on Computational Bioengineering, Compiègne, 6-8 September 2017 (Oral presentation). 


\section{Contents}

1 Background 29

1.1 Clinical motivation .................. 33

1.2 Cardiovascular Magnetic Resonance Imaging . . . . . . . . . . 35

1.2.1 Magnetic Resonance Imaging . . . . . . . . . . . . 37

1.2.2 Cardiac MRI . . . . . . . . . . . . . . . 40

1.2.3 Spatial resolution . . . . . . . . . . . . 43

1.2.4 Temporal resolution . . . . . . . . . . . . . 44

1.2.5 Sources of errors in MRI . . . . . . . . . . . . 45

1.2.6 Impact of 4D Flow CMR limits on the flow descriptors . . . 46

1.3 Cardiovascular Computational Fluid Dynamics . . . . . . . . . . 48

1.3.1 Fluid Dynamics . . . . . . . . . . . . . . . . 49

1.3.2 Cardiovascular CFD . . . . . . . . . . . 52

1.3.3 CFD and PC MRI: results comparison . . . . . . . . . . 55

1.4 Particle Image Velocimetry . . . . . . . . . . . . . . . 59

1.4.1 Principle ....................... 59

1.4.2 PIV in cardiovascular imaging as a validation tool . . . . 62

1.5 Data driven computational approaches . . . . . . . . . . . 65

1.6 Summary of the first chapter . . . . . . . . . . . . . . 67

$2 \quad$ Aim and Objectives $\quad 68$

3 EMRI framework and preliminary testing $\quad 70$

3.1 EMRI framework . . . . . . . . . . . . . . . . . 70 
3.1.1 Computational aspects . . . . . . . . . . 72

3.2 Numerical verification . . . . . . . . . . . . 72

3.2.1 Enhancement with different sampling grids . . . . . . . 74

3.2.2 Enhancement with different force parameter $\mathrm{k} \ldots \ldots . . .75$

3.2.3 Enhancement in presence of noise . . . . . . . . . 75

3.2.4 Temporal persistence of the enhancement . . . . . . . . 76

3.2.5 Results and discussion . . . . . . . . . . . 76

3.2.6 Enhancement with different sampling grids . . . . . . . . 76

3.2.7 Enhancement with different force parameter k . . . . . . . 79

3.2.8 Enhancement in presence of noise . . . . . . . . . . 82

3.2.9 Temporal persistence of the enhancement . . . . . . . . 84

3.3 2D EMRI . . . . . . . . . . . . . . 85

3.3.1 4D Flow CMR data processing . . . . . . . . . . . 85

3.3.2 Materials and methods . . . . . . . . . . . . 87

3.3.3 Results and discussion . . . . . . . . . . . . . . . 89

3.3.4 MRI coordinate mapping . . . . . . . . . . . . . . 89

4 Validation of EMRI: assessment of the flow field in a $U$ bend using 4D Flow CMR, CFD, 2D PIV and EMRI 94

4.1 Material and Methods . . . . . . . . . . . . . . . . . 95

4.1 .1 Test rig ........................ 95

4.1.2 PIV Experimental set-up . . . . . . . . . . . . . . . . . 99

4.1 .3 4D Flow CMR . . . . . . . . . . . . . . 105

4.1 .4 EMRI and CFD . . . . . . . . . . . . . . . . 106

4.2 Results and discussion . . . . . . . . . . . . . . . 108

4.2 .1 Velocity maps . . . . . . . . . . . . . 111

4.2.2 Velocity profiles . . . . . . . . . . . . 112

4.2 .3 Cross sectional flow . . . . . . . . . . . . . 114

4.3 Discussion and limitation of the study . . . . . . . . . . . 115 
5 3D EMRI: patient-specific application

5.1 Methods . . . . . . . . . . . . . . . . . . 135

5.1.1 Magnetic resonance imaging and post processing . . . . . . 135

5.1.2 Enhanced Magnetic Resonance Imaging . . . . . . . . . 140

$5.1 .3 \quad$ CFD . . . . . . . . . . . . . . 142

5.1.4 Sets of simulations conducted . . . . . . . . . . 143

5.2 Results and discussion . . . . . . . . . . . . . . . . . 144

5.2.1 Aortic flow at late systole: comparison between CFD steady state, EMRI and 4D Flow CMR flow fields. . . . . . . . . . 144

5.2.2 Aortic flow at late systole: comparing standard EMRI and EMRI Wall function . . . . . . . . . . . . 152

5.2.3 Aortic flow studied with EMRI wall function and time resolved CFD . . . . . . . . . . . . . . . 155

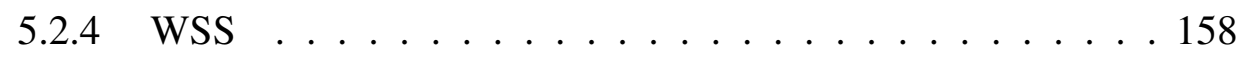

5.2.5 Short segment of the descending aorta . . . . . . . . . 160

5.3 Final remarks and limitations $\ldots \ldots \ldots 161$

6 Application of EMRI in pathophysiological research: atherogenesis 193

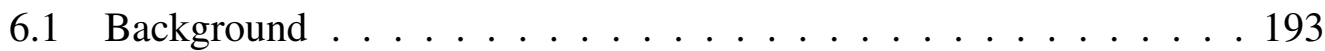

6.2 Hypothesized role of temperature in atherogenesis . . . . . . . . 195

6.3 The carotid bifurcation . . . . . . . . . . . . . . 197

6.3.1 MRI experimental setup . . . . . . . . . . . . . . . 199

6.3.2 Results and discussion . . . . . . . . . . . 205

6.4 Translation to animal study: preliminary results . . . . . . . . . . 209

6.5 Limitations . . . . . . . . . . . . . . . . . 210

7 Conclusions and future work $\quad 213$

$\begin{array}{lr}\text { Appendices } & 218\end{array}$

$\begin{array}{lrr}\text { A Grid independence tests } & \mathbf{2 1 8}\end{array}$

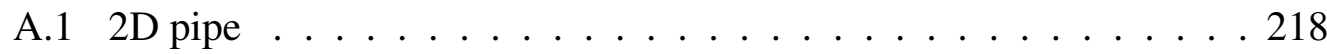




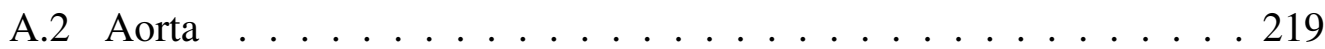

A.3 U bend . . . . . . . . . . . . . . . . . . 219

A.4 EMRI model of carotid bifurcation . . . . . . . . . . . . . . 221 


\section{List of Figures}

1.1 Leonardo da Vinci, cardiovascular system and principle organs of a woman, 1509-10. . . . . . . . . . . . . 29

1.2 The haemodynamics of the aortic root: (a) eddys in Valsalva sinuses in a drawing from Leonardo's notebook (19083 Verso, copyright reserved by Her Majesty Queen Elizabeth ZI.) (b) 4-dimensional flow magnetic resonance imaging streamline visualization of aortic root of a volunteer $[4] . \ldots \ldots \ldots$

1.3 Demonstration of the one way action of the valves in the veins in the the circulation of the blood from De Motu Cordis in 18th-century Latin edition of the anatomical works of William Harvey 'Exercitatio anatomica de motu cordis et sanguinis in animalibus' [5] . . . . 31

1.4 Pie chart showing the causes of death (in \%) in Europe (source https://ec.europa.eu/eurostat). . . . . . . . . . . . . 33

1.5 Cardiovascular MRI: a transversal slice of the ascending (AAO) on the left, and descending aorta (DAO), on the right, of an healthy

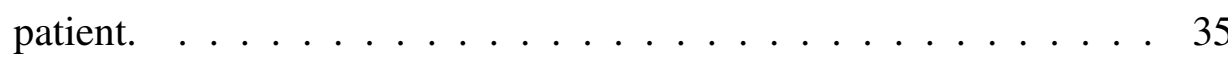

1.6 4D flow MRI with streamlines, of a patient with bicuspid aortic valve showing the flow paths in the ascending aorta and postcoarctation segment (adapted from Stankovic et al. [6]). . . . . . . . 36

1.7 Effect of a spatial varying gradient on the resonance frequency. (adapted from Netter, F. H. (2010). [7]) _ . . . . . . . . . . . . . 39 
1.8 Slice selection process: when a spatial varying gradient $G_{z}$ is applied, the total magnetic field experienced by a proton and its resonance frequency depend on its position $z_{i}$. The slice thickness $\Delta z$ is determined by the amplitude of $G_{z}$ and by the bandwidth $\Delta \omega$ [8]. . . 39

1.9 Visualization of the flow in the aorta using flow estimated by 4D flow MRI Arterys Inc, San Francisco. . . . . . . . . . . . . . 40

1.10 (a) Segmentation of the lumen of 4D Flow CMR data of an aorta, and corresponding velocity map ; (b) B-spline interpolation of the measured velocities, used to derive continuous flow velocities and velocity derivatives along the segmentation contour (adapted from A.F. Stalder et al. [9]). . . . . . . . . . . . . . . . . . . . 48

1.11 Visualization of the flow patterns of a patient specific CFD model of the aortic arch

1.12 Blood-flow patterns at peak systole in the major cerebral arteries of a subject obtained by MRI data and CFD model (adapted from Cebral et al. [10]) . . . . . . . . . . . . . . . . . 56

1.13 Wall shear stress (WSS) in a patient aneurysm in systolic phase computed with CFD (on the left) and PC-MRI (on the right) (adapted from Boussel et al. [11]). . . . . . . . . . . . . . . . . 57

1.14 Streamlines and in-plane velocity distributions in one plane of the systolic peak in the healthy adult aorta measured with PC-MRI and Laminar CFD model (adapted from Miyazaki et al. [12]). . . . . . . 58

1.15 Localized Normalized Helicity at late systole in a patient specific aorta CFD model with full (3D) and axial (1D) blood velocity field prescribed as inlet boundary condition (adapted from Morbiducci et al. $[13]) \ldots \ldots \ldots \ldots \ldots \ldots \ldots$

1.16 A 2D PIV experimental apparatus. . . . . . . . . . . . . . 60

1.17 In-plane velocity estimated by a laminar CFD mode and measured with PIV in two planes of a TCPC model (adapted from Pekkan et al. $[14]) \ldots \ldots \ldots \ldots \ldots$. . . . . . . . . . . . . . . 62 
1.18 Velocity fields for steady measurement in the cerebral aneurysm. (a) MRI and (b ) particle image velocimetry (PIV) velocity vectors; (d) MRI and (e) computational fluid dynamics (CFD) in plane velocity vectors images in the slice indicated by the red arrow in the top row (adapted from Van Ooji et al. [15]. . . . . . . . . . . . . . . . . 64

3.1 Diagram showing the EMRI workflow. . . . . . . . . . . 71

3.2 Quad mesh of the 2D channel. . . . . . . . . . . . . . . 74

$3.3 \mathrm{a}), \mathrm{b}), \mathrm{c}), \mathrm{d})$ Velocity profiles at $\mathrm{y}=0.003 \mathrm{~m}$ computed with different grids at which the experimental velocity $\mathrm{v}_{\exp }$ was sampled; the lines ' $\mathrm{v}_{\exp }$ interpolation' indicate the interpolation of the experimental points on the mesh and the 'no force' lines are the velocity profile in the case without external force e) squared velocity difference between the parabolic profile and the computed ones with different grids of sampling points and f) root mean squared difference. 77

3.4 Velocity (a) and pressure (b) along the axis of the channel for the different grids at which the experimental velocity $\overrightarrow{\exp }$ was sampled, and with the case without enforcement. . . . . . . . . 78

3.5 Force residuals maps in a portion of the 2D surface computed for the different grids at which the experimental velocity $v_{e} x p$ was sampled. 78

3.6 a) Velocity profiles at $\mathrm{y}=0.025 \mathrm{~m}$, calculated for the different grids at which the experimental velocity $\mathrm{v}$ was given. b) Percentage difference between the velocity profiles with various sampling point densities at $\mathrm{y}=0.025 \mathrm{~m}$ and the case with no force $\ldots \ldots . . .80$

3.7 Velocity difference maps in a portion of the 2D surface computed at different values of $\mathrm{k} \ldots \ldots \ldots \ldots$

3.8 Kinetic energy of the system (a) and number of convergence iterations at different values of $k \ldots \ldots \ldots$. . . . . . . . 82

3.9 Pressure drop along the axis of the channel at different values of $\mathrm{k}$

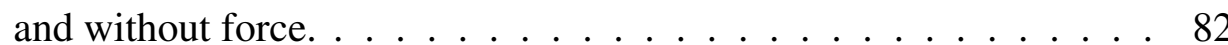


3.10 Velocity profiles obtained 'experimentally' and computed with EMRI in the developed flow area $(y=0.025 \mathrm{~m})$ in the case with SNR 20 (a) and $30 \mathrm{~dB}(\mathrm{~b}) . \ldots \ldots \ldots \ldots$

3.11 (a) Velocity profiles in the developed flow area $(y=0.025 \mathrm{~m})$ computed with EMRI in the case without noise, and with SNR 20 and $30 \mathrm{~dB}$; (b) velocity difference between the velocity computed with EMRI with noise and without noise for the two values of SNR. . . .

3.12 (a) Pressure drop along the axis of the channel in the case without noise and for SNR 20 and $30 \mathrm{~dB}$ (b) difference in the pressure drop along the axis of the channel between the case without noise and the case with noise (SNR 20 and $30 \mathrm{~dB}) \ldots \ldots \ldots$. . . . . . 84

3.13 (a) Velocity value on the axis of the channel and (b) velocity profiles in the non developed flow region $(\mathrm{y}=0.003 \mathrm{~m})$ at $t_{2}$ (last time step with the enforcement) and at 10 time steps following the removal of the external force. . . . . . . . . . . . . . . 84

3.14 Slice of the 4D Flow CMR showing the magnitude (a), and the three phase encoding anterior- posterior (AP) (b), right-left (RL) (c) and feet-head $(\mathrm{FH})(\mathrm{d}) \ldots \ldots \ldots \ldots \ldots$. . . . . . . . . . 86

3.15 Slice of the MRI with the mask (in blue) of the region on which the study was performed. . . . . . . . . . . . . . 88

3.16 Velocity waveform of the aorta at the inlet and instant selected for the $2 \mathrm{D}$ analysis. . . . . . . . . . . . . . . . . 89

3.17 (a) Plot of the mapped MRI data (indicated as MRI data) and the mesh nodes of the CFD model. (b) Velocity maps retrieved directly form the MRI data (indicated as data) and velocity maps interpolation on the domain of the CFD model. . . . . . . . . . . . . . 90

3.18 Velocity maps of the region of the descending aorta computed with the MRI, CFD and EMRI.

3.19 Velocity maps with vectors of the region of the descending aorta computed with the MRI, CFD and EMRI. 
3.20 Section of the aortic portion (a) where the velocity profiles measured with 4D Flow CMR, and computed CFD and EMRI where compared. . . . . . . . . . . . . . . 92

3.21 Velocity difference map between the 4D Flow CMR measured velocity and the one computed with EMRI. . . . . . . . . . . . 92

4.1 Geometry and PVA mould of the U bend pipe. . . . . . . . . . . . . 96

4.2 Schematics of the casting : the PVA model is enclosed in a box and afterwards the silicone is poured, which results in a hollow structure once the material is dissolved. . . . . . . . . . . . . . . 97

4.3 A silicone phantom used for testing purposes before (a) and after (b) the refractive index matching. The square grid at the back shows the optical distortions due to the difference in the refractive index of the silicone and the surrounding fluid. (c) U bend phantom filled with the working fluid with the same refractive index of the silicone, with the square grid at the back, showing the absence of optical distortions. 98

4.4 U bend silicone phantom without (on the left) and with (on the right) refractive index matched fluid. . . . . . . . . . . . . . . 100

4.5 Diagram of the experimental rig. . . . . . . . . . . . . 100

4.6 Phantom with the plane selected for the comparison. . . . . . . 101

4.7 Diagram of the piv experimental setup. . . . . . . . . . . . . . 101

4.8 U bend with the 9 regions of interest imaged separately during the PIV experiment. . . . . . . . . . . . . . . . 102

4.9 Photo of the blood pump and of the MRI control room during the

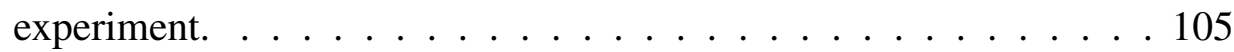

4.10 PIV raw data (a), processed vectors (b) of the region II of the $\mathrm{U}$ bend, with a magnification of one portion of it (c). . . . . . . . . . 119

4.11 Slice of the 4D Flow CMR showing the magnitude (M), and the three phase encoding anterior-posterior (AP), right-left (RL) and feet-head $(\mathrm{FH})$ at the time instant 2 . . . . . . . . . . . . . 120 
4.12 Post processed 4D Flow CMR data in the coronal plane (middle plane of the $U$ bend) at the time instant 2: (a) velocity map and (b) velocity vectors overlaid on the velocity contour plot. . . . . . . . 121

4.13 Average velocity at the inlet of the $U$ bend during the pump cycle measured with 4D Flow CMRI (with dashed line representing the average velocity ) and PIV (with standard deviation, at the four instants of the cycle considered for the comparison). . . . . . . . . 121

4.14 Velocity maps in the selected plane at the 4 instant measured with 4D Flow CMRI and PIV and computed with CFD and EMRI. . . . . 122

4.15 Three sections of the $\mathrm{U}$ bend where the velocity profiles are compared.123

4.16 Velocity profiles in section $\mathrm{A}$ at the 4 instant of time considered, measured with 4D Flow CMRI and PIV (with standard deviation) and computed with CFD and EMRI. . . . . . . . . . . . . . . 124

4.17 Velocity profiles in section B at the 4 instant of time considered, measured with 4D Flow CMRI and PIV (with standard deviation) and computed with CFD and EMRI. . . . . . . . . . . . 125

4.18 Velocity profiles in section $\mathrm{C}$ at the 4 instant of time considered, measured with 4D Flow CMRI and PIV (with standard deviation) and computed with CFD and EMRI. . . . . . . . . . . . 126

4.19 Relative difference between PIV and CFD, and PIV and EMRI velocity magnitudes $(v)$ in section A at the 4 instant of time considered. 127

4.20 Relative difference between PIV and CFD, and PIV and EMRI velocity magnitudes $(v)$ in section B at the 4 instant of time considered. 128

4.21 Relative difference between PIV and CFD, and PIV and EMRI velocity magnitudes $(v)$ in section $\mathrm{C}$ at the 4 instant of time considered. 129

4.22 Cross sections of the $\mathrm{U}$ bend at $0^{\circ}$ and $180^{\circ} \ldots \ldots \ldots$

4.23 Velocity maps in the cross sectional plane at $0^{\circ}$ at the 4 instants measured with 4D Flow CMRI and computed with CFD and EMRI. 131

4.24 Velocity maps in the cross sectional plane at $180^{\circ}$ at the 4 instants measured with 4D Flow CMRI and computed with CFD and EMRI. 132 
5.1 A model of the human aorta. . . . . . . . . . . . . . . . 133

5.2 3D geometry of the aorta. . . . . . . . . . . . . 136

5.3 Average velocity at the inlet of the aorta, at BCA, LCCA and LSCA during the cardiac cycle. . . . . . . . . . . . . . . . 137

5.4 Average velocity at the inlet of the aorta with standard deviation and the instants selected for the comparison of 4D Flow CMR, CFD and EMRI (blue dots). . . . . . . . . . . . . . . . . . . . 138

5.5 Raw 4D Flow CMR velocity profile and processed one at $\mathrm{t}=0.2348$ $\mathrm{s}$ from the beginning of the systole, computed on the line crossing the descending aorta (on the right of the picture). . . . . . . . . 139

5.6 Velocity surfaces computed from raw 4D Flow CMR velocity profile and processed one at $\mathrm{t}=0.2348 \mathrm{~s}$ from the beginning of the systole, in a transverse plane of the descending aorta (shown Figure 5.10 b). . . . . . . . . . . . . . . . 140

5.7 Diagram showing the raw velocity measured with 4D Flow CMR (right end side) in a transversal cross section of a patient specific aorta (left hand side), with the segmentation profile of the vessel overlapped. . . . . . . . . . . . . . . . . 141

5.8 Geometry of the aorta used for the CFD simulation. . . . . . . . . 143

5.9 Portion of the aorta geometry considered and boundary conditions applied. . . . . . . . . . . . . . . . 144

5.10 Sections considered to compare the flow path in the aorta: a) transverse sections at the inlet and outlet of the aortic arch, b) transverse section in the descending aorta and c) sagittal section of the whole aorta. ........................... 145

5.11 Velocity maps of the aorta ( $t=0.2348 s$ ) computed with 4D Flow CMR, CFD and EMRI. Sagittal cutting plane (top left) and transversal cross section (bottom left). The locations of cutting planes are shown on the left panel. . . . . . . . . . . . . . . . . . . . 146 
5.12 Velocity maps of the aorta $(t=0.2348 s)$ of the Sagittal cutting plane (top row) and transversal cross section (bottom row) computed with 4D Flow CMR and EMRI, with a magnification of a portion of the descending aorta and of a portion near the wall. . . . . . . . . . 147

5.13 Velocity difference between EMRI and 4D Flow CMR in the sagittal section of the aorta $(\mathrm{t}=0.2348 \mathrm{~s})$ with an area magnified. . . . . 148

5.14 Velocity difference between EMRI and 4D Flow CMR in a transverse section of the aorta $(\mathrm{t}=0.2348 \mathrm{~s})$ with two area magnified $(\mathrm{a}, \mathrm{b}) .148$

5.15 Pressure maps of the aorta $(t=0.2348 \mathrm{~s})$ on the wall with CFD and EMRI . . . . . . . . . . . . . . . . . . . . 149

5.16 Wall shear stress maps of two sides of the aorta $(t=0.2348 \mathrm{~s})$ computed with 4D Flow CMR, CFD and EMRI. . . . . . . . . . 150

5.17 Diagram showing the trend of $\phi\left(d_{W A L L}\right)$ with $\triangle$ Pix $(\mathrm{mm})$ of 0.75 $\mathrm{mm}$ in the sagittal cutting plane (on the left) and transversal cross section (on the right) of a patient specific aorta . . . . . . 153

5.18 Raw 4D Flow CMR velocity profile and processed one at $\mathrm{t}=0.2348$ $\mathrm{s}$ from the beginning of the systole, computed on the line crossing the descending aorta (on the right of the picture) . . . . . . . 154

5.19 Velocity surfaces computed from raw 4D Flow CMR data $\left(v_{M R I}\right)$, from the $\left(\phi\left(d_{W A L L}\right) \cdot v_{M R I}\right)$ and from EMRI data at $\mathrm{t}=0.2348 \mathrm{~s}$ from the beginning of the systole, in a transverse plane of the descending aorta (shown Figure 5.10 b) . . . . . . . . . . . . . . 154

5.20 Velocity maps of the aorta $(\mathrm{t}=0.2348 \mathrm{~s})$ computed with EMRI and EMRI with Wall function. Sagittal cutting plane (top row) and transversal cross section (bottom row). The locations of cutting planes are shown on the left panel. . . . . . . . . . . . . . . . 164

5.21 Velocity maps of the aorta ( $\mathrm{t}=0.2348 \mathrm{~s})$ computed with EMRI and EMRI Wall function in the transversal cross section (see Figure 5.20) with a magnification of an area near the wall. . . . . . . 165 
5.22 Velocity difference $\left(\mathrm{v}-\mathrm{v}_{\text {MRI }}\right)$ maps of the aorta $(\mathrm{t}=0.2348 \mathrm{~s})$ computed with EMRI and EMRI Wall function in the sagittal cutting plane (first row) and transversal cross section (second row) (the sections are showed on the left side of the picture) $\ldots \ldots \ldots 6$

5.23 Wall shear stress maps of two sides of the aorta $(t=0.2348 \mathrm{~s})$ computed with EMRI and EMRI Wall function. . . . . . . . . . 167

5.24 Velocity maps of the aorta at early systole (point 1) computed with CFD, EMRI (Wall function) and measured with 4D Flow CMR in the sagittal cutting plane of the aorta (Figure $5.10 \mathrm{c}$ ). . . . . . . 168

5.25 In plane velocity vector maps of the aorta at early systole (point 1) computed with CFD, EMRI (Wall function) and measured with 4D Flow CMR in the sagittal cutting plane of the aorta (Figure $5.10 \mathrm{c}$ ). . 169

5.26 In plane velocity vector maps of the aortic arch at early systole (point 1) computed with CFD, EMRI (Wall function) and measured with 4D Flow CMR in the sagittal cutting plane of the aorta (Figure

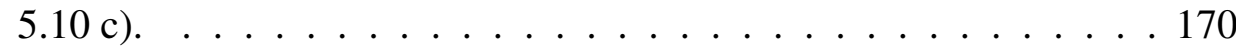

5.27 Velocity maps of the aorta at early systole (point 1) computed with CFD, EMRI (Wall function) and measured with 4D Flow CMR in the transversal cross section of the aorta (Figure $5.10 \mathrm{~b}$ ). . . . . 171

5.28 In plane velocity vector maps of the aorta at early systole (point 1) computed with CFD, EMRI (Wall function) and measured with 4D Flow CMR in the transversal cross section of the aorta (Figure 5.10

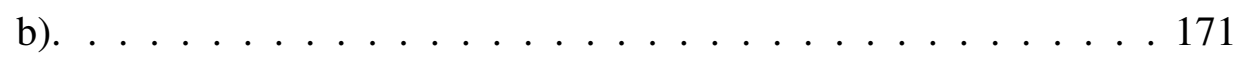

5.29 In plane velocity vector maps of the aorta at early systole (point 1) computed with CFD, EMRI (Wall function) and measured with 4D Flow CMR in the transversal cross sections of the aortic arch (Figure 5.10 a). . . . . . . . . . . . . . . 172

5.30 Velocity maps of the aorta at peak systole (point 2) computed with CFD, EMRI (Wall function) and measured with 4D Flow CMR in the sagittal cutting plane of the aorta (Figure $5.10 \mathrm{c}$ ). . . . . . . 173 
5.31 In plane velocity vector maps of the aorta at peak systole (point 2) computed with CFD, EMRI (Wall function) and measured with 4D Flow CMR in the sagittal cutting plane of the aorta (Figure $5.10 \mathrm{c}$ ). . 174

5.32 In plane velocity vector maps of the aortic arch at peak systole (point 2) computed with CFD, EMRI (Wall function) and measured with 4D Flow CMR in the sagittal cutting plane of the aorta (Figure $5.10 \mathrm{c})$.

5.33 Velocity maps of the aorta at peak systole (point 2) computed with CFD, EMRI (Wall function) and measured with 4D Flow CMR in the transversal cross section of the aorta (Figure $5.10 \mathrm{~b}$ ). . . . . . 176

5.34 In plane velocity vector maps of the aorta at peak systole (point 2) computed with CFD, EMRI (Wall function) and measured with 4D Flow CMR in the transversal cross section of the aorta (Figure 5.10

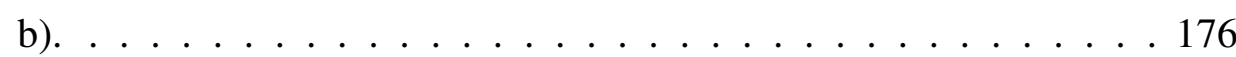

5.35 In plane velocity vector maps of the aorta at peak systole (point 2) computed with CFD, EMRI (Wall function) and measured with 4D Flow CMR in the transversal cross sections of the aortic arch (Figure 5.10 a) . . . . . . . . . . . . . . . . 177

5.36 Velocity maps of the aorta at late systole (point 3) computed with CFD, EMRI (Wall function) and measured with 4D Flow CMR in the sagittal cutting plane of the aorta (Figure $5.10 \mathrm{c}$ ). . . . . . 178

5.37 In plane velocity vector maps of the aorta at late systole (point 3) computed with CFD, EMRI (Wall function) and measured with 4D Flow CMR in the sagittal cutting plane of the aorta (Figure $5.10 \mathrm{c}$ ). . 179

5.38 In plane velocity vector maps of the aortic arch at late systole (point 3) computed with CFD, EMRI (Wall function) and measured with 4D Flow CMR in the sagittal cutting plane of the aorta (Figure 5.10

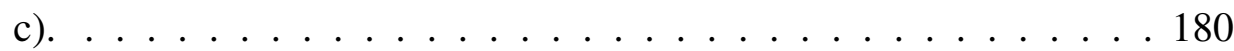


5.39 Velocity maps of the aorta at late systole (point 3) computed with CFD, EMRI (Wall function) and measured with 4D Flow CMR in the transversal cross section of the aorta (Figure $5.10 \mathrm{~b}$ ). . . . . . 181

5.40 In plane velocity vector maps of the aorta at late systole (point 3) computed with CFD, EMRI (Wall function) and measured with 4D Flow CMR in the transversal cross section of the aorta (Figure 5.10

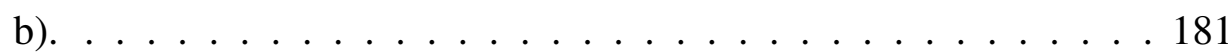

5.41 In plane velocity vector maps of the aorta at late systole (point 3) computed with CFD, EMRI (Wall function) and measured with 4D Flow CMR in the transversal cross sections of the aortic arch (Figure 5.10 a). . . . . . . . . . . . . . . 182

5.42 Velocity maps of the aorta at mid diastole (point 4) computed with CFD, EMRI (Wall function) and measured with 4D Flow CMR in the sagittal cutting plane of the aorta (Figure $5.10 \mathrm{c}$ ). . . . . . . 183

5.43 In plane velocity vector maps of the aorta at mid diastole (point 4) computed with CFD, EMRI (Wall function) and measured with 4D Flow CMR in the sagittal cutting plane of the aorta (Figure $5.10 \mathrm{c}$ ). . 184

5.44 In plane velocity vector maps of the aortic arch at mid diastole (point 4) computed with CFD, EMRI (Wall function) and measured with 4D Flow CMR in the sagittal cutting plane of the aorta (Figure

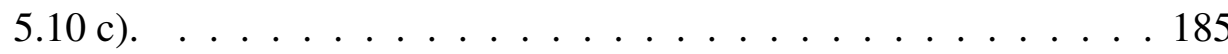

5.45 Velocity maps of the aorta at mid diastole (point 4) computed with CFD, EMRI (Wall function) and measured with 4D Flow CMR in the transversal cross section of the aorta (Figure $5.10 \mathrm{~b}$ ). . . . . 186

5.46 In plane velocity vector maps of the aorta at mid diastole (point 4) computed with CFD, EMRI (Wall function) and measured with 4D Flow CMR in the transversal cross section of the aorta (Figure 5.10

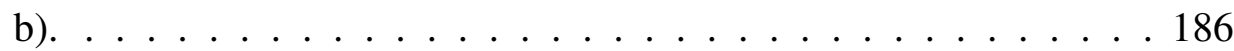


5.47 In plane velocity vector maps of the aorta at mid diastole (point 4) computed with CFD, EMRI (Wall function) and measured with 4D Flow CMR in the transversal cross sections of the aortic arch (Figure 5.10 a) . . . . . . . . . . . . . . . . 187

5.48 WSS maps of the two sides of the aorta at early systole (point 1) computed with CFD, EMRI (Wall function) and computed with 4D Flow CMR. . . . . . . . . . . . . . . . . . . . . 188

5.49 WSS maps of the two sides of the aorta at peak systole (point 2) computed with CFD, EMRI (Wall function) and computed with 4D Flow CMR . . . . . . . . . . . . . . . . . . . . . 189

5.50 WSS maps of the two sides of the aorta at late systole (point 3) computed with CFD, EMRI (Wall function) and computed with 4D Flow CMR. . . . . . . . . . . . . . . . . . . . . 190

5.51 WSS maps of the two sides of the aorta at mid diastole (point 4) computed with CFD, EMRI (Wall function) and computed with 4D Flow CMR. . . . . . . . . . . . . . . . . . . . . . 191

5.52 Normalized WSS retrieved from 4D Flow MRI, CFD and EMRI velocity data in the selected points of the cardiac cycle. . . . . . . . 192

5.53 Velocity map of the longitudinal and cross-sectional planes of the aorta $(\mathrm{t}=0.2348 \mathrm{~s})$ computed for a short segment using standard CFD and EMRI, and entire aorta with EMRI . . . . . . . . . . . 192

6.1 Temperature maps at steady state on the surface of a Vena 8 Microfluidic Biochip, a Vena Delta Y1 Microfluidic Biochip and $\mu$ Slide y-shaped channel (Unpublished data, courtesy of LVTS, INSERM) 
6.3 a) PLA model of the carotid bifurcation; b)temperature map at the steady state in the carotid bifurcation phantom, measured with IRC; c) Graph showing the temperature change in time of three areas marked in b), in the transitional phase and in the steady state (Unpublished data, courtesy of LVTS, INSERM). . . . . . . . . . . . 199

6.4 Bruker transmit-receive volume coil . . . . . . . . . . . . . 200

6.5 a) $3 \mathrm{D}$ geometry of the bifurcation phantom $\mathrm{b}$ ) $3 \mathrm{D}$ printed phantom c) experimental setup for MRT: the 3D printed phantom was filled up with an hydrogel to enhance MRI signal and avoid noise on the wall of the bifurcation; moreover in order to monitor the temperature, a temperature probe was inserted. . . . . . . . . . . . . 200

6.6 Resin phantom and Agarose phantom phase images: transverse (a) and coronal view (b). The red dotted line is showing the vessel wall. 201

6.7 Development of vascular phantom for MRT: 1) outer box design and printing in resin; 2) lumen mold printing in PVA; 3) phantom resulting after the casting with agarose gel; 4) phantom obtained after the dissolution of the lumen mould in water (the blue dotted line is showing the vessel wall); 5) phantom enclosed in the resin box.202

6.8 Geometry of the carotid artery and on the surrounding box. . . . . . 203

6.9 Velocity maps of the carotid bifurcation computed with 4D Flow CMR and EMRI. Sagittal cutting plane (top row) and transversal cross section (bottom row). The locations of cutting planes are shown on the left panel. . . . . . . . . . . . . . . 206

6.10 Temperature maps of the box surrounding the carotid bifurcation computed with MRT and EMRI. Sagittal cutting plane (top row) and transversal cross section (bottom row). The locations of the planes are shown on the left panel. . . . . . . . . . . . 207

6.11 WSS maps of the carotid bifurcation phantom, computed with 4D Flow CMR (on the left )and EMRI (on the right). The two sides of the vessel are shown. . . . . . . . . . . . . . . . . . 208 
6.12 EMRI temperature maps computed on an surface at $1 \mathrm{~mm}$ offset from the lumen of the bifurcation model. . . . . . . . . . . . . . 208

6.13 Contour plot of the Nusselt number on the two sides of the carotid bifurcation wall. . . . . . . . . . . . . . . . 209

6.14 Streamlines in the carotid bifurcation with velocity map overlaid. . . 210

6.15 a) ApoE $-/-$ mouse prepared for the scanning. b) Bruker MRI scanner used for the acquisition. . . . . . . . . . . . . . 210

6.16 Magnitude (3D volume visualization) and the MRT phase maps. The black dotted line in the MRT maps shows the shimmed area. The left subclavian artery bifurcation MRT map is magnified on the right side of the picture. . . . . . . . . . . . . . . 211

A.1 (a) section where the velocity profiles where computed; (b) velocity profiles computed with the different meshes. . . . . . . . . . . . 218

A.2 Plot of the peak velocity computed on the selected line and the number of elements of the meshes. . . . . . . . . . . . . . . . 219

A.3 (a) section where the velocity profiles where computed; (b) velocity profiles computed with the different meshes. . . . . . . . . . . . 220

A.4 Plot of the peak velocity computed on the selected line and the number of elements of the meshes. . . . . . . . . . . . . 220

A.5 (a) section where the velocity profile was computed; (b) velocity profiles computed with the different meshes . . . . . . . . . . . 221

A.6 Plot of the average velocity computed on the selected line and the number of elements of the meshes. . . . . . . . . . . . . . . 221

A.7 (a) section where the temperature and velocity profiles where computed; (b) velocity and (c) temperature profiles computed with the different meshes . . . . . . . . . . . . . . . . 222

A.8 Plot of the peak velocity (a) and peak temperature (b) computed on the selected line and the number of elements of the meshes. . . . . . 222 


\section{List of Tables}

3.1 Energy and energy change (\%) for the different grids at which the experimental velocity $v_{\text {exp }}$ was sampled . . . . . . . . 75

3.2 Energy and energy change (\%) for the different grids at which the experimental velocity $v_{\exp }$ was sampled $\ldots \ldots . . . . . . .79$

3.3 Energy and duration of the simulation change (\%) at different values

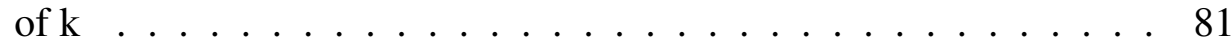

4.1 Gain scaling $s$ at the different instants of the pump cycle considered. 107

$4.2 \Delta t$ (the laser delay), $\varepsilon_{P I V}$ (the maximum velocity uncertainty) and $\varepsilon_{\text {piv }}$ of the PIV measurements at the different instants of the pump cycle . . . . . . . . . . . . . . . . 110

4.3 SNR, VNR and $\sigma_{v}$ of the 4D Flow CMR data at the different instants of the pump cycle considered. . . . . . . . . . . . 110

4.4 $\Delta_{P I V-C F D}$ for each section at the different instants of the pump cycle considered. . . . . . . . . . . . . . . . 113

4.5 $\Delta_{P I V-E M R I}$ for each section at the different instants of the pump cycle considered. . . . . . . . . . . . . . . . 113

4.6 $\Delta_{M R I-P I V}$ for each section at the different instants of the pump cycle considered. . . . . . . . . . . . . . . . . . 114

4.7 $\Delta$ for each time instant of the pump cycle considered, averaged over the sections considered. . . . . . . . . . . . . . . . 114

5.1 SNR,VNR and $\sigma_{v}$ of the 4D Flow CMR data at the 4 instants considered of the cardiac cycle. . . . . . . . . . . . . . . . 139 
5.2 Gain scaling $s$ at the considered instants of the heart cycle. . . . . 142

5.3 Quantities used to assess the impact of the introduction of $\phi\left(d_{W A L L}\right)$ for different $\Delta P i x$ values. . . . . . . . . . . . . . . . . . . 147

5.4 Quantities used to assess the impact of the introduction of $\phi\left(d_{W A L L}\right)$ for different $\Delta$ Pix values. . . . . . . . . . . . . . . . . 152

5.5 WSS computed from processed 4D Flow CMR data, time resolved CFD and EMRI, averaged over the aortic wall for the considered instants of the cardiac cycle. . . . . . . . . . . . . . . . . 159

A.1 Characteristics of the different meshes of the 2D pipe . . . . . 218

A.2 Characteristics of the different meshes of the aorta. . . . . . . . 219

A.3 Characteristics of the different meshes of the $U$ bend. . . . . . . . . 220

A.4 Characteristics of the different meshes of the agarose model of the carotid bifurcation. . . . . . . . . . . . . . . 222 


\section{Chapter 1}

\section{Background}

"Panta rhei."

(everything flows)

Heraclitus of Ephesus

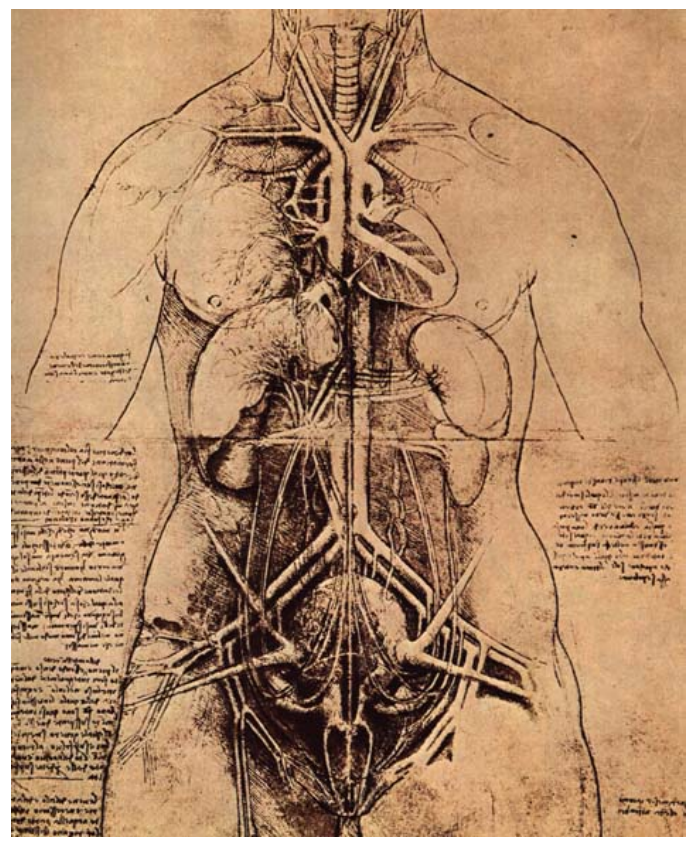

Figure 1.1: Leonardo da Vinci, cardiovascular system and principle organs of a woman, 1509-10.

The role of cardiovascular system as the main way in which the body was nourished was known since ancient Greece (400 BC). Aristotle (384 BC) under- 
stood that a disruption in this nutritive process played a major role in disease, and that the heart was at the centre of this mechanism. The first model of the circulatory system was hypothesized by Erasistratus (300 BC), who considered the heart to be the source of arteries (that would contain air) and veins. Galen (129 AD) synthesized all the Greek knowledge, performed experiments and studies on dead or living animals, and was the first one to prove that arteries contain blood.

After the fall of the Roman Empire (500-1400 AD), the church played a central role in the scholarly tradition and the focus was mainly to preserve the Ancient Greek teachings, harmonizing them with theology. The first dissection of human body appeared in the 13th century at the University of Bologna, with the aim of confirming the findings of Galen.

a

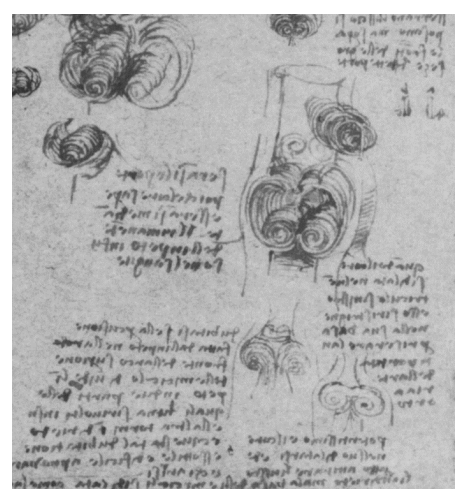

b

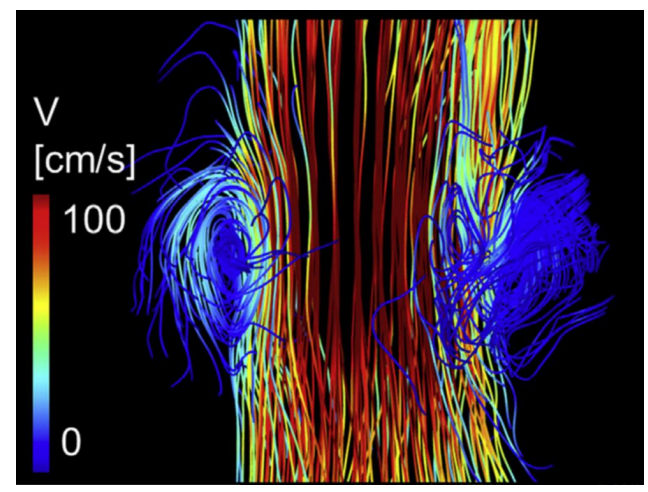

Figure 1.2: The haemodynamics of the aortic root: (a) eddys in Valsalva sinuses in a drawing from Leonardo's notebook (19083 Verso, copyright reserved by Her Majesty Queen Elizabeth ZI.) (b) 4-dimensional flow magnetic resonance imaging streamline visualization of aortic root of a volunteer [4].

It was the Italian Renaissance that brought breakthrough discoveries, starting with Leonardo da Vinci (1452 - 1519) that was the first one to make accurate drawing of the heart, with atria and valves. A Leonardo's drawing depicting the human anatomy, including the vascular system, is shown in Figure 1.1.

Among his important contributions there is also the identification of vortex formation in the aortic root sinuses as a mechanism for the valve's closure (Figure $1.2 \mathrm{a})$. An in-vivo confirmation of his finding had to wait for the use of flow sensitive 
magnetic resonance imaging to map the velocity field inside the Valsalva sinuses (Figure 1.2 b) [4].

The pulmonary circulation of blood was theorized by Realdo Colombo (1516 - 1559), where the venous blood in the right ventricle passes through the lungs into the left ventricle and arteries, creating a circulatory system model which is an hybrid between closed (pulmonary) and open (systemic) circulation.

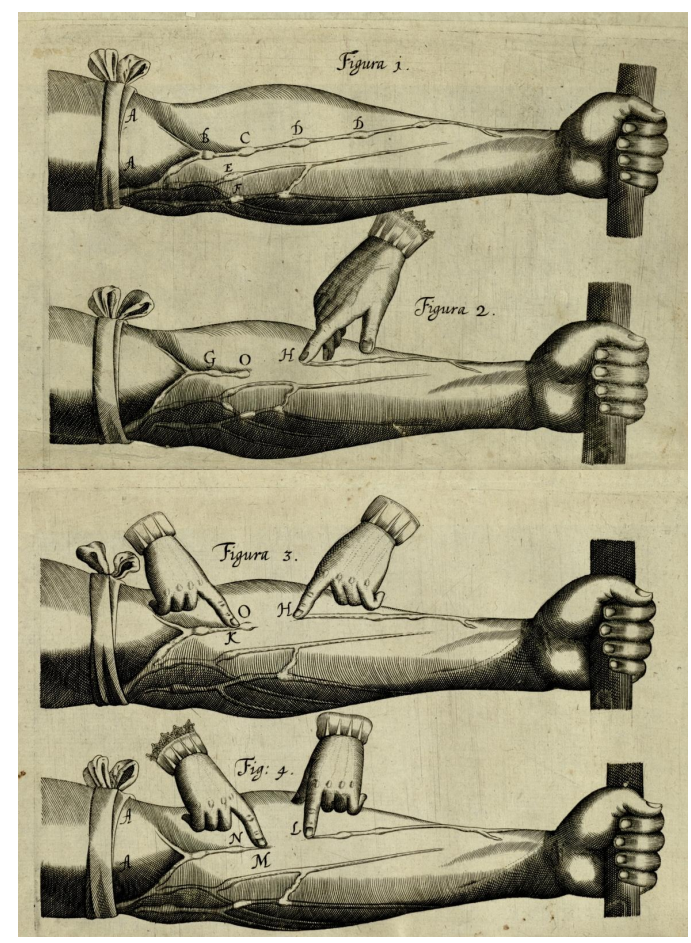

Figure 1.3: Demonstration of the one way action of the valves in the veins in the the circulation of the blood from De Motu Cordis in 18th-century Latin edition of the anatomical works of William Harvey 'Exercitatio anatomica de motu cordis et sanguinis in animalibus' [5].

It was only in 1628, that William Harvey (1578 - 1657), an English clinicianscientist, published his work Exercitatio anatomica de motu cordis et sanguinis in animalibus (which translates in Anatomical exercises on the motion of the heart and blood in animals) in which a closed circulatory system was presented. Figure 1.3, a picture from Harvey's anatomical work, shows the demonstration of blood flow in the venus system.

Harvey discovered that blood circulates around the whole body and not only in the lungs, quoting his words: 
'It has been shown by reason and experiment that blood by the beat of the ventricles flows through the lungs and heart and is pumped to the whole body. There it passes through pores in the flesh into the veins through which it returns from the periphery everywhere to the centre, from the smaller veins into the larger ones, finally coming to the vena cava and right atrium. This occurs in such an amount, with such an outflow through the arteries and such a reflux through the veins, that it cannot be supplied by the food consumed. It is also much more than is needed for nutrition. It must therefore be concluded that the blood in the animal body moves around in a circle continuously and that the action or function of the heart is to accomplish this by pumping. This is only reason for the motion and beat of the heart.'

Thanks to Harvey's findings, light was shed on the blood circulation mechanisms, that had eluded many scientists till 1600 . He is remembered as the father of modern physiology.

The human cardiovascular system, whose main components are the heart and the blood vessels, includes a pulmonary circulation, a pathway through which the blood is oxygenated, and a systemic circulation, that allows the blood to provide oxygen and nourishing to the rest of the body.

The heart is the pump of the cardiovascular system and has one atrium and one ventricle for each circulation. In particular the right atrium collects the deoxygenated blood from the superior and the inferior venae cavae, that would be then passed to the right ventricle to be pumped through the pulmonary artery to the lungs, to receive oxygen and release carbon dioxide. On the contrary the left atrium receives the oxygenated blood from the pulmonary veins, and then it is passed to the left ventricle that pumps it through the aorta to the systemic circulation. The aorta, the biggest artery of the human body, branches out into smaller arteries, arterioles and capillaries, in order to supply with oxygen and nutrients all the body. The blood leaving the capillaries is deoxygenated and rich in cell waste products, and travels through venules and veins to reach the superior and inferior vena cavae, and finally the right heart. 


\subsection{Clinical motivation}

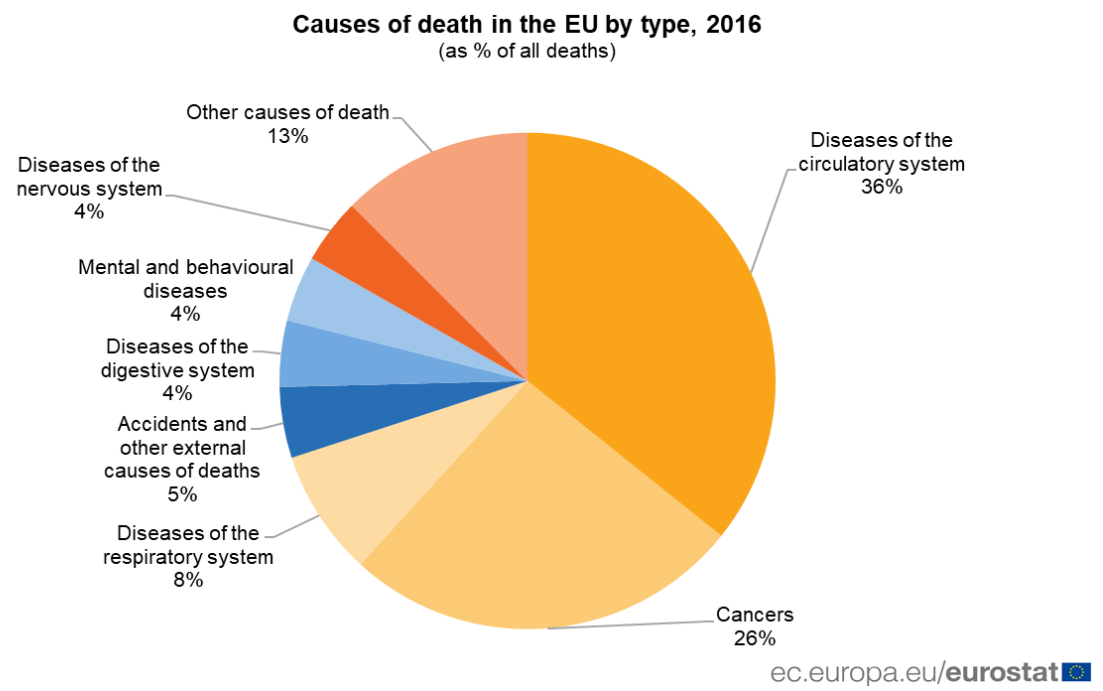

Figure 1.4: Pie chart showing the causes of death (in \%) in Europe (source https://ec.europa.eu/eurostat).

Cardiovascular Diseases (CVDs) are a group of conditions and disorders that affect the heart and the blood vessels, and include: hypertension, coronary heart diseases, cerebro-vascular diseases, peripheral vascular diseases, heart failure, rheumatic heart diseases, congenital heart diseases and cardio-myopathies.

CVDs are the major causes of death globally, accounting for $31 \%$ of fatalities in 2016 [5]. $85 \%$ of these deaths are due to heart attack and stroke. Over the past two decades, the rates of mortality due to CVDs have declined substantially in highincome countries and with no doubt this is the results of prevention and individual health-care intervention strategies [5].

Most of the CVDs can be in fact prevented, by tackling the behavioural risks factors (e.g. unhealthy diet, obesity, smoking of tobacco, physical activity, etc.) and some medical conditions (e.g. hypertension, diabetes, hyperlipidaemia, etc.), in which CVDs have their roots. The main alarm bells are high blood pressure [16], elevated blood glucose [17], overweight and obesity [18].

Unfortunately, although early detection and diagnosis would enable more effective therapeutic interventions, early stage CVDs are often undetected because they are asymptomatic. Consequently, there is a pressing need for novel markers 
which could predict the development of a cardiovascular disease and reduce the occurrence of cardiovascular events.

In current practice, functional assessments of CVDs are carried out only for specific applications, such as the transvalvular pressure drop to quantify heart valves' stenosis, or the fractional flow reserve to assess functional severity of coronary artery stenosis. Efforts have also been made to predict long-term vascular remodelling, such as development of atherosclerotic plaque or aneurysms, estimating more difficult-to-measure parameters e.g. wall shear stress (WSS), a descriptor of the force acting on the vessel walls proven to alter the cells physiology and determine the onset of some CVDs [19, 20].

Haemodynamics is also a crucial factor in aortopathies, where a thoracic aortic enlargement could progress to the life-threatening complication of acute aortic dissection. For example in the pathogenesis of aortic enlargement of bicuspid aortic valve patient, a problem still controversial as two distinct theories exist. A genetic theory, seeing the aortic wall weakness as a genetic developmental result affecting both the aortic valve and the aorta, and an haemodynamic one, linking the aortic enlargement to the abnormal stress on the aortic wall generated by turbulent flow and eccentric jets characterizing bicuspid aortic valve patients [21, 22].

The effect of disturbed flow patterns on the cardiac function has also been investigated in patients with abnormal anatomies or those who underwent surgical interventions. An example is given by patients with single ventricle heart defects, palliated with a Fontan procedure, that passively directs the venous blood to the pulmonary circulation to cope with the absence of a ventricle. In these cases the effort to reduce energy loss with favourable haemodynamics led to an alternative approach to the classical Fontan operation, with the introduction of the total cavopulmonary connection (TCPC) and the removal of the right atrium, part of the standard approach [23].

Haemodynamics has been proved to be vital for prosthetic devices implantation, such as aortic valve replacement. In these case the characteristics and location of the vortex in the Valsalva sinuses has a crucial impact on the performances of 
the device implanted and the restoration of the physiological flow in the aortic root [24].

Hence, the knowledge of the flow characteristics and their changes over time could offer an important support to the understanding, prevention and diagnosis of CVDs and provide an assessment of the outcome of a surgical intervention in terms of "flow restoration" [25].

\subsection{Cardiovascular Magnetic Resonance Imaging}
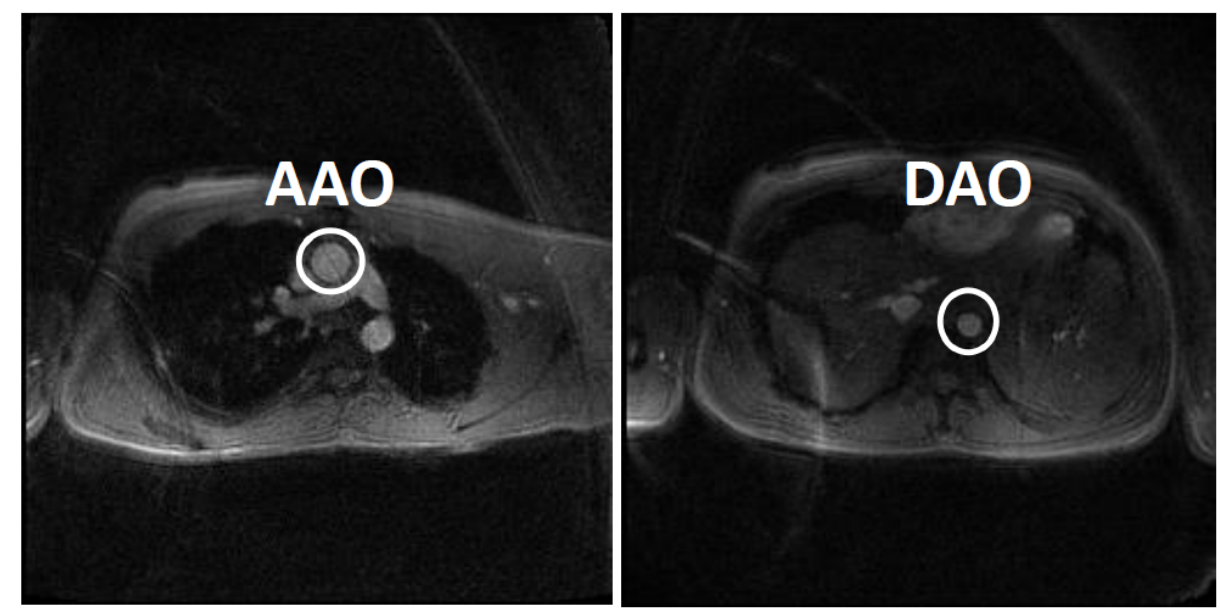

Figure 1.5: Cardiovascular MRI: a transversal slice of the ascending (AAO) on the left, and descending aorta (DAO), on the right, of an healthy patient.

Diagnosis and risk assessment of CVDs in clinical environment could be performed using multiple direct techniques such as magnetic resonance imaging (MRI), computed tomography $(\mathrm{CT})$, ultrasound. MRI, thanks to its non invasiveness, is widely used by clinicians as a tool for assessing CVDs, monitor their evolution and the treatment efficacy [6]. Figure 1.5 shows two slices of an MRI scanning of the descending and ascending aorta of an healthy volunteer.

During the last decade technical developments of MRI, allowed access to not only morphological information on the cardiovascular anatomy but also functional information, such as blood flow or cardiac perfusion, allowing a more thorough assessment of CVDs. The introduction of phase contrast MRI (PC-MRI) in the 1980s, allowing only a 2D velocity evaluation, and its development allowing a time- 
resolved (CINE) three dimensional (3D) PC-MRI, which is referred to as 4D Flow CMR [26], has granted a broad clinical acceptance of this technique as a valuable resource for the visualization and quantitative evaluation of blood flow in the heart, aorta and large vessels.

Promising results have been achieved in investigating blood flow patterns in healthy aorta, allowing the visualization of the sequential development of axial, helical and retrograde flow [27]. Flow sensitive MRI has been used also to show the abnormal flow patterns in the descending aorta and a marked helical flow paths of patients with aortic coarctation [28] or bicuspid aortic valve (Figure 1.6) [6].

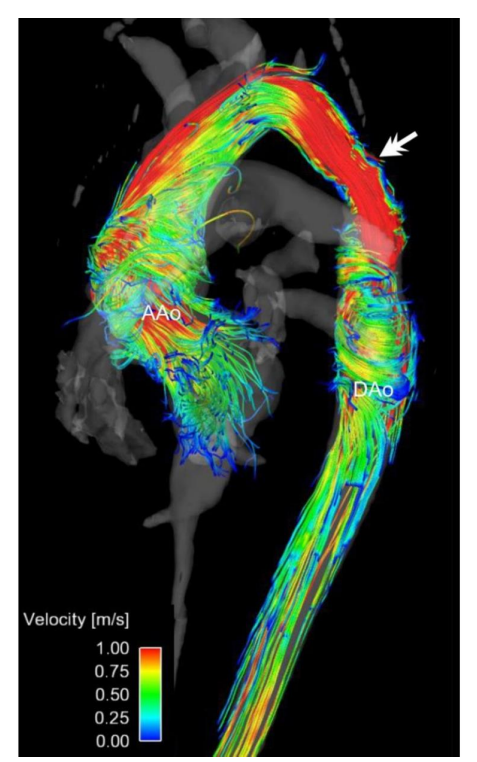

Figure 1.6: 4D flow MRI with streamlines, of a patient with bicuspid aortic valve showing the flow paths in the ascending aorta and post-coarctation segment (adapted from Stankovic et al. [6]).

Moreover 4D Flow CMR enabled improvements upon the haemodynamic assessment of diseases, allowing to access flow derived metrics such as wall shear stress (WSS). For example this parameter is potentially related with the occurrence of CVDs in patients with bicuspid aortic valve, where abnormally high values of WSS ( 1.96 standard deviation higher than volunteers) on the greater curvature of the ascending aorta, are associated with tissue remodelling [29]. Pressure gradients have also been calculated using MR-based three-directional velocity vector fields, for instance in the aorta [30] and in the left atrium and left ventricle [31]. 
Among the other parameters estimated via velocity sensitive MRI there are volumetric flow (cardiac output, valve regurgitation), peak blood velocity (for stenosis), patterns and timings of velocity waveforms, and flow and pressure distributions within heart chambers (abnormal ventricular function) and vessels (pulse-wave velocity, vessel wall disease) [32]. However the limited spatio-temporal resolution affects the accuracy of the estimate of MRI-based haemodynamic parameters [33].

Firstly in order to understand the limits of 4D Flow CMR, the basis of this technique will be introduced, and afterwards the limitations of this approach.

\subsubsection{Magnetic Resonance Imaging}

MRI is a non invasive imaging tool able to provide images of human body. The main source of MR signals are hydrogen nuclei, abundant in the human body in form of water and fat. These nuclei are characterised by an intrinsic form of angular momentum called spin. At room temperature no net magnetic moment is present, due to the thermal random motion. It is therefore necessary to apply an external magnetic field.

Hydrogen, in presence of an external magnetic field, has two energy states, $\pm \frac{1}{2}$, often referred to as parallel $(+)$ (or spin up) and anti-parallel $(-)$ (or spin down) with respect to external magnetic field. A transition between these two levels could happen if there is an absorption or an emission of an electromagnetic radiation with frequency $\omega_{0}$, so that the resonance condition is met (equation 1.1):

$$
\omega_{0}=\frac{\Delta E}{\hbar}=\frac{\gamma \hbar B_{0}}{\hbar}
$$

where $\Delta E$ is the energy difference between the two states, $\hbar$ is the Plank constant $\left(6.62607004 \cdot 10^{-34} \mathrm{~kg} \cdot \mathrm{m}^{2} \cdot \mathrm{s}^{-1}\right), \gamma$ is the gyromagnetic ratio and $B_{0}$ is the main magnetic field.

At body temperature, with a magnetic field of 1.5 Tesla there is one extra spinup proton in $10^{6}$. If we assume a magnetic field $B_{0}$ to be along the $\mathrm{z}$-axis, the $\mathrm{x}$ and y component of the magnetisation $\bar{M}$ are zero, since a net magnetisation will be present only in the z-direction. 
Since at thermal equilibrium the number of spin up is bigger than the number of spin down, $\bar{M}$ points along the positive direction of the $\mathrm{z}$-axis. Unfortunately it is not possible to measure $\bar{M}$ when it is aligned with the magnetic field $\overline{B_{0}}$, since the magnitude of $\overline{B_{0}}$ is substantially bigger than the magnetisation of the spin population in that direction. Nevertheless, each magnetic moment vector has a non-zero transverse component, and coherence in this direction could be achieved applying a magnetic field $B_{1}$, perpendicular to the main magnetic field. However, in order to resonate with the system, the frequency of $B_{1}$ must be equal to $\omega_{0}$.

$B_{1}$ is referred to as an radio frequency pulse, since the associated frequency is in the range of the radio frequency. When an external magnetic field $\overline{B_{0}}$ is applied to a population of magnetic moments, a torque is produced, and $\bar{M}$ precesses about $\overline{B_{0}}$ at angular frequency $\omega_{0}$, the well known Larmor frequency.

The result of this precession (called forced precession) is that the vector $\bar{M}$ is tipped onto the $\mathrm{x}-\mathrm{y}$ plane, and can be therefore measured. Indeed by placing RF-receive coils in the x-y plane, the time varying magnetisation vector $\bar{M}$ induces a voltage proportional to $M_{x y}$. This signal is named free induction decay, since it decays exponentially as a consequence of the magnetisation relaxation.

\subsubsection{Encoding information about the position of all the different spins}

By introducing spatial varying magnetic fields, it is possible to spatially encode the MR signal. In order to do that, gradient coils are used; they produce a linearly varying magnetic field across the volume to be imaged. The gradient separates spins according to their positions (figure 1.7) by making their resonance frequency vary with position.

The data is acquired in the so called k-space (frequency space) and Fourier transformed in order to obtain the image in the physical space. Usually Fast Fourier Transform (FFT) is used because of its computational efficiency. The MR signal could be acquired in a 3D k-space. Nevertheless most techniques reduce the problem to two dimensions, exploiting a slice selecting gradient (usually in the zdirection). 


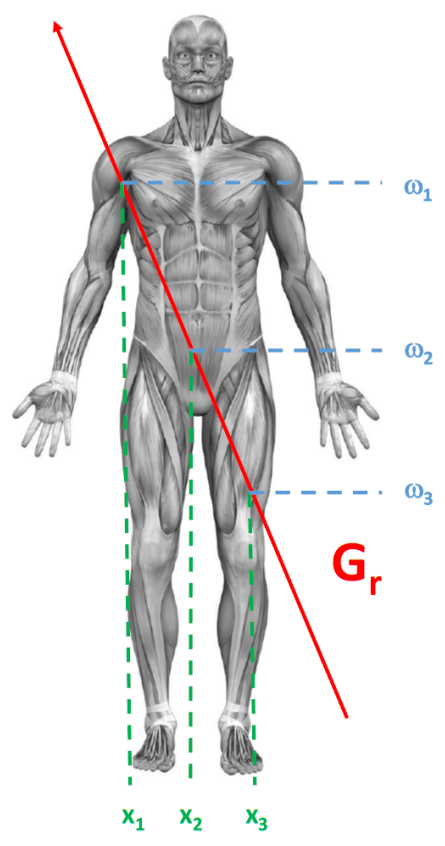

Figure 1.7: Effect of a spatial varying gradient on the resonance frequency. (adapted from Netter, F. H. (2010). [7])

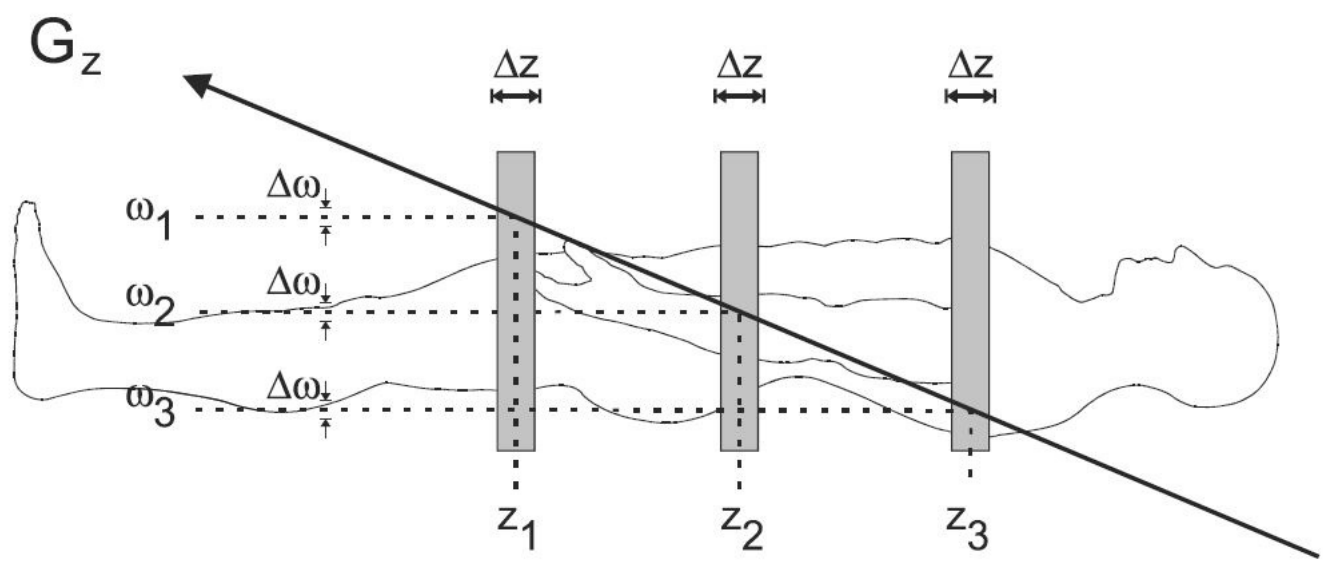

Figure 1.8: Slice selection process: when a spatial varying gradient $G_{z}$ is applied, the total magnetic field experienced by a proton and its resonance frequency depend on its position $z_{i}$. The slice thickness $\Delta z$ is determined by the amplitude of $G_{z}$ and by the bandwidth $\Delta \omega[8]$.

\subsubsection{Excite selectively a 2D slice in the object to be imaged}

If we apply an RF pulse with a limited range of frequency components at the same time as a gradient along the $\mathrm{z}$ direction $G_{z}$, only the nuclei that are resonating with 
the bandwidth of the RF pulse will be excited (figure 1.8).

The central frequency of the RF-pulse and the gradient determines the location excited: changing the central frequency of the pulse changes the slice position. Whereas the bandwidth of the pulse regulates the slice thickness, together with the gradient amplitude $G_{z}$.

So far it has been shown how the 3D problem has been simplified to a $2 \mathrm{D}$ one, using the slice selection process. In Cartesian MR imaging the data is acquired moving in the k-space row by row. In order to do so, after a slice select gradient (applied on the $\mathrm{z}$-axis), two other gradients are applied in $\mathrm{x}$ and $\mathrm{y}$ axis.

Firstly a phase encoding gradient is applied on the y-axis: this gradient allows the trajectory in the k-space to move along the y direction, therefore up and down. The second gradient, so called readout or frequency encoding gradient is instead applied on the $\mathrm{x}$-axis, in order to move the trajectory in the $\mathrm{x}$-direction, thus left and right in the $\mathrm{k}$-space. At the same time of the readout gradient the data is acquired.

\subsubsection{Cardiac MRI}

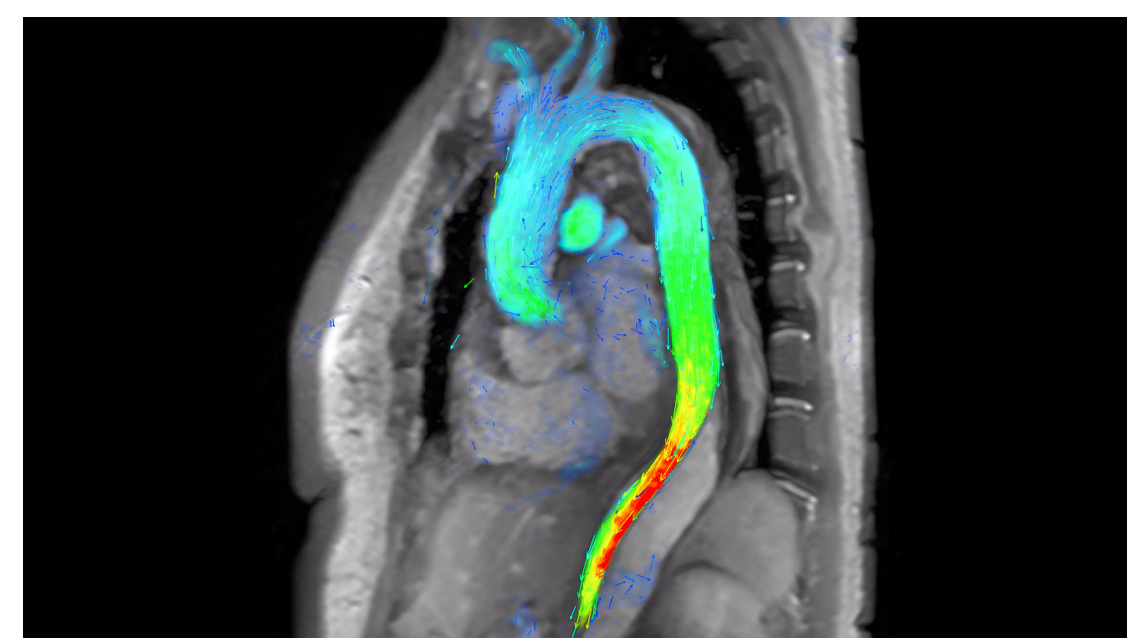

Figure 1.9: Visualization of the flow in the aorta using flow estimated by $4 \mathrm{D}$ flow MRI Arterys Inc, San Francisco.

The strong correlation between disturbed flow patterns and the development of vascular diseases created a huge interest in studying the blood flow in the human vascular system, and in particular in the heart [25].

MRI is the gold standard method of measuring blood flow in the clinical envi- 
ronment. This sensitivity to flow can be better understood by examining the formulation of the received MRI signal $S(t)$ at time $t$ (Equation 1.2):

$$
S(t)=\iiint \rho(\bar{r}) \exp \left(-j \gamma \bar{G}_{r} \cdot \bar{r} t\right) d \bar{r}
$$

It can be seen that the rate at which the spins dephase depends on their position (in 3D space) and the amplitude of the gradient waveforms $\left(G_{r}\right)$ at time $\tau$. Therefore, spins accumulate a phase over time depending on their location, according to the Equation 1.3:

$$
\phi(t)=\gamma \int_{t_{0}}^{t} \bar{G}(\tau) \bar{r}(\tau) d \bar{\tau}
$$

Performing a Taylor expansion of this equation demonstrates that in fact the phase of an MR signal is proportional to a spins position, velocity, acceleration, jerk, and higher orders of motion (Equation 1.4):

$$
\begin{aligned}
\phi(t) & =\gamma \overline{r_{0}} \int_{t_{0}}^{t} \bar{G}(\tau) d \tau+\gamma \overline{v_{0}} \int_{t_{0}}^{t} \bar{G}(\tau) \tau d \tau+\ldots+\gamma \frac{1}{n !} \int_{t_{0}}^{t} \bar{G}(\tau) \tau^{n} d \tau= \\
& =\gamma\left(\overline{r_{0}} M_{0}+\overline{v_{0}} M_{1}+\overline{a_{0}} M_{2}+\ldots \frac{1}{n !}\left(\frac{d^{n} r}{d t^{n}}\right)_{t=0} M_{n}\right)
\end{aligned}
$$

where the $n^{t h}$-order moment of a gradient $G, M_{n}$ is defined as (Equation 1.5):

$$
M_{n}=\int_{t_{0}}^{t} \bar{G}(\tau) \tau^{n} d \tau
$$

From Equation 1.4 it can be seen that the velocity of a spin is proportional to $M_{1}$ (Equation 1.6):

$$
M_{1}=\int_{t_{0}}^{t} \bar{G}(\tau) \tau d \tau
$$

Velocity can be measured using velocity encoded phase-contrast magnetic resonance (PC-MR) imaging. PC MRI can be used for pressure estimation, however as all measurements are affected by noise, the differentiation of velocity causes a strong amplification of the velocity noise itself.

4D Flow CMR refers to PC MR with flow-encoding in all three spatial directions (3D) along the cardiac cycle (+1D), resulting in 4D. 
In order to do so, the phase of the MRI signal is exploited to encode velocity and acceleration of the blood in the cardiovascular system. Let us rewrite Equation 1.4 up to the second order of $n$ (Equation 1.7):

$$
\phi(t)=\gamma\left(\overline{r_{0}} M_{0}+\overline{v_{0}} M_{1}+\overline{a_{0}} M_{2}+\ldots\right)
$$

If we assume no acceleration or higher order motion quantities, this equation becomes (Equation 1.8):

$$
\phi(t)=\gamma\left(\overline{r_{0}} M_{0}+\overline{v_{0}} M_{1}\right)
$$

It is possible to remove the effect of displacement of the spins from the phase of the signal with an ad-hoc sequence design so that $M_{0}=0$. As a consequence (Equation 1.9):

$$
\phi(t)=\gamma \overline{v_{0}} M_{1}, \quad M_{0}=0
$$

From Equation 1.9, it is possible to see that the phase encoded is proportional to the velocity of the spins. This assertion applies if we neglect the phase offsets coming from additional sources, such as $\overline{B_{0}}$ inhomogeneities, susceptibility effects, Eddy currents (for details see section 1.2.5). In order to remove these additional phase offsets two measurements are made, with different first order moment, and subsequently they are subtracted. This approach enables to remove the background offsets. The phase difference could be expressed as (Equation 1.10):

$$
\Delta \phi(t)=\gamma \overline{v_{0}} \Delta M_{1}
$$

where $\Delta M_{1}$ is the difference between the first order moments of the two measurements. This approach allows to determine the velocity of the spins, and it is called velocity encoding $v_{E N C}$. A spin that travels at velocity $\pm v_{E N C}$ will cause a shift in the phase signal of $\pm \pi$ radians (Equation 1.11):

$$
v_{E N C}=\frac{\pi}{\gamma \Delta M_{1}}
$$

The most common approach is the so called asymmetric encoding In this case one 
measurement is acquired with velocity compensated gradients $\left(M_{1}=0\right.$, thus $v_{E N C}=$ 0 ) and the other with velocity encoded gradient (desired $M_{1}$, thus desired $v_{E N C}$ ).

In order to collect data during in-vivo imaging, usually the acquisition has to be triggered by means of an electrocardiogram waveform. In cases where triggering is not possible, the acquisition method is called real-time. With respect to conventional gated MRI, in which the k-space is covered during cardiac cycles thanks to a trigger, real time sequences do not use any triggering. Therefore they exploit highly under sampled imaging techniques in order to acquire the k-space in single shots. The clinical reason for these sequences is to accommodate the needs of ill patients that could not handle long breath-holding. In fact a single image could be acquired in less than $100 \mathrm{~ms}$. The main drawbacks of real time PC MRI are the low temporal and spatial resolutions [34].

\subsubsection{Spatial resolution}

MRI spatial resolution is defined by the size of the voxels of the image. Usually the resolution is different in each direction. Therefore voxels are often not cubes. The size of a voxel is related to the matrix size, the field of view (FOV) and the slice thickness. Usually the spatial resolution in clinical cardiovascular MRI is $1-2 \mathrm{~mm}$ [35].

The matrix size is given by the number of phase encodings in one direction and the number of frequency encodings in the other one. Increasing them improves the spatial resolution. Increasing the frequency encoding step or readout steps is related to the sampling rate of the scanner, therefore does not affect the duration of the scanning. Contrarily increasing the phase encoding steps increases accordingly the time of the acquisition. The FOV is the area scanned during the acquisition, hence if this is divided by the matrix size, the voxel size is obtained [36].

The depth of the voxel is determined by the slice thickness. Usually this is the largest dimension of the voxel, due to the fact that the thicker the slice the higher the signal to noise ratio (SNR). It is usually two or three times larger than the in-plane encoding direction. Therefore the resolution is quite poor in this direction.

Slice thickness might have a significant impact on flow parameter assessments 
for example when it comes to give an estimate of the jet velocities, since it could give rise to velocity underestimations [37].

Scanning with high resolution is really important considering that, the lower the resolution, the higher the partial volume effect [8]. In order to explain this concept let us consider a voxel that contains the amount $f_{A}$ and $f_{B}$ of two materials $A$ and $B$. The MRI signal of the entire voxel $S_{\text {voxel }}$ will be given by the Equation 1.12:

$$
S_{\text {voxel }}=f_{A} S_{A}+f_{B} S_{B}
$$

where $S_{A}$ and $S_{B}$ are the MRI signals of the tissue A and B respectively. As a consequence the contrast of the tissue A (or tissue B) is reduced accordingly to the fraction of the voxel it occupies. Reducing the voxel size reduces the partial volume effect, since most of the pixel is more likely to be made out of only one type of tissue.

When it comes to the phase of the signal, the voxel dimension is really important for a voxel may contain a range of velocities [11]. In fact this voxel could contain different phase-shifts and as a consequence the signal measured is only an average of all the single signals in the voxel. Therefore the velocity measured could be inaccurate [38].

To minimise this error to no more than $10 \%$, it is necessary to have at least four pixels across the vessel diameter [37].

\subsubsection{Temporal resolution}

The main features of flow compensated sequences with asymmetric encoding are the presence of more gradients than usual, and the fact that two acquisitions are needed, one phase compensated and one with phase encoding. An advantage of this approach is to reduce flow artefacts. However in comparison with standard sequences, the additional gradients lead to increased echo and repetition time.

In cardiac MRI, temporal resolution is chosen such that cardiac cycle is covered by approximately $15-20$ time frames. The acquisition consists of at least four data sets for each directional velocity encoding [8]. Doing so allows to average the 
velocities of each time point of the cycle. Moreover this procedure increases the SNR. In fact the SNR improves as the square root of the number of acquisitions (if the noise is un-correlated from one experiment to the next) [8].

A standard sequence normally requires about 15-20 minutes [39], and 8-12 minutes per scan even using a fast sequence [40] (spatial resolution can be improved by increasing the acquisition time). As a consequence, the flow captured is affected by the physiological beat-to-beat variability of the flow field during the acquisition, that could impair the accuracy of the velocity field encoded. The fact that the acquisition comes out at a certain interval implies that the exact time of peak velocity may be missed and the velocity is therefore usually underestimated [41].

\subsubsection{Sources of errors in MRI}

Together with the resolution limitations, another source of inaccuracy, in the estimate of the velocities through MRI, is given by the intrinsic errors caused by the physics behind this imaging modality [11]. The major source of errors include Maxwell terms, gradient field distortions and eddy current effects.

However there are some strategies that could help in reducing them and enable a more accurate flow quantification.

\subsubsection{Maxwell Terms}

Whenever a magnetic field gradient is switched on, additional unwanted non linear magnetic field are generated. This results in phase errors and therefore in inaccurate velocity measurements [42]. It is possible to implement some strategies in order to correct or reduce these effects. Some of them have to be implemented on the gradient design, resulting however in longer acquisition. Other corrections are performed during the post processing on the image itself.

\subsubsection{Gradient field distortions}

Significant image artefacts are caused by the non uniformity of the magnetic field gradients. These imperfections could cause lots of errors in the estimate of velocity fields. In fact, any inaccuracy in the gradient strength results in a wrong velocity encoding [43]. 
A substantial improvement in the velocity quantification, could be achieved by correcting the phase acquired with the phase shift caused by the bias field. For instance a phantom with steady flow could be used to determine this phase shift, that is then subtracted from the phase of the acquisition of interest. However, this approach is sometimes non-practical since a bias field estimate has to be done for each scan in advance. More recent methods exploit statistical approaches or non parametric automatic algorithms, executed without user interaction [44].

\subsubsection{Eddy currents}

During an MRI acquisition, when magnetic gradients are switched on, the magnetic flux undergoes variations. Hence some currents, called eddy currents, are induced in parts of the scanner (e.g. coils) and in the patient [8]. Therefore the desired gradients strength and duration are altered and, as a consequence, spatially varying phase errors affect the acquisitions. Subtraction of two acquisitions eliminates shifts due only to static field inhomogeneities.

However during flow sensitive MRI, different gradients waveforms used for the subsequent velocity encoding, lead to different eddy current-induced phase shifts. Therefore phase shifts present in the subtraction image could not be due to the flow [45].

Corrections for these phase changes are based for example on subtraction of the estimate of eddy current phase shifts. Usually the region containing static tissue is used, and a plane (or higher order polynomial) is fitted to the phase difference data, and then subtracted from the image.

\subsubsection{Impact of 4D Flow CMR limits on the flow descriptors}

The limited resolution of 4D Flow CMR has limited its application to the heart [46], aorta [47] and large arteries [48]. Nevertheless all the aforementioned limitations of MRI lead to inaccuracies in the reconstructed velocity field, which, in turn, result in non-conformance with some fluid dynamics principles such as mass conservation and divergence free velocity fields.

The first one is caused by the fact that errors in the velocity lead to different 
flow rates at different cross sections. In the second case the inaccuracy in the velocity vectors directions could cause some streamlines to stop on the walls of the region of interest [41].

One of the limits of the 4D Flow CMR sequences is the definition of the velocity encoding $\left(v_{E N C}\right)$. If $v_{E N C}$ is set too high this can cause phase wrapping, if it is set too low then it results in poor sensitivity to low velocities [46]. This aspect is very important when velocity fields are used to compute WSS, as in the case when the velocity gradient in the proximity of the wall is computed. This flaw adds up to the limited spacial resolution that make these regions highly susceptible to velocity estimation errors.

To compensate for these inaccuracies cumbersome post processing is necessary [49]. For example B-spline interpolation of the velocity field could provide continuous velocity and derivatives on the vessel wall; this is the case of the example shown in Figure 1.10, where this interpolation is applied to 4D Flow CMR of an aorta [9]. Another common approach is to assume the velocity on the vessel surface to be zero, and then apply linear interpolation in the normal direction towards the centre of the vessel [12].

Even when considering big vessels, such as the aorta, complex flow patterns may not be captured directly and to be visualized might require post-processing to allow for a quantitative assessment of flow features [50].

Markl et al. [51] showed also problems with the reproducibility of flow and wall shear stress using flow sensitive MRI in the aorta. In particular they point out that the correct estimation of absolute WSS might be problematic and that only the relative distribution of wall-related parameters might be reliable. These inaccuracies originate partly from the low or zero velocity components missed or added due to segmentation errors, but also from the calculation of the local velocity derivative at the vessel lumen boundary.

Also flow paths are susceptible to 4D Flow CMR limitations. In fact Hope et al. [52] used 4D Flow CMR to assess the flow patterns characterizing ascending aortic aneurysms. This study shows how the flow in patients with aneurysms was char- 


\section{a}

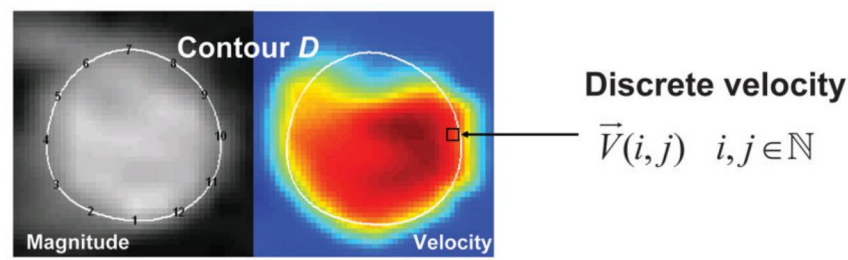

b

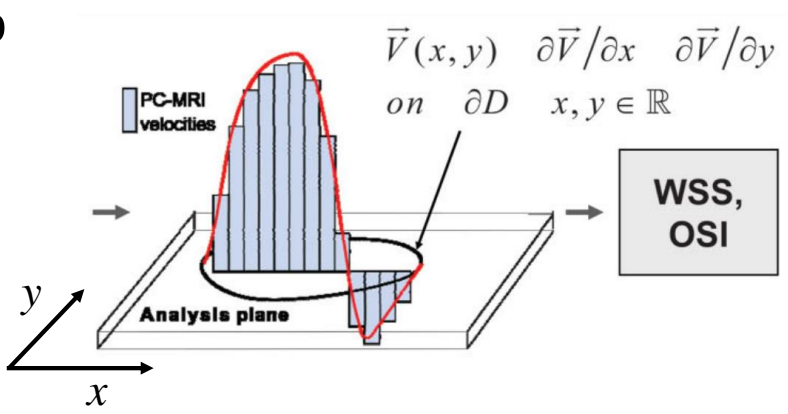

Figure 1.10: (a) Segmentation of the lumen of $4 D$ Flow CMR data of an aorta, and corresponding velocity map ; (b) B-spline interpolation of the measured velocities, used to derive continuous flow velocities and velocity derivatives along the segmentation contour (adapted from A.F. Stalder et al. [9]).

acterized by larger helices and long lasting retro-grade flow. Moreover an increase in the average velocity between the ascending and descending aorta characterized the aneurysm patients compared to the volunteers. However the limited spatial and temporal resolution of the 4D Flow CMR sequence limited a correct estimation of flow patterns at shorter time scales and therefore impacted the measurements of peak systolic velocities.

These limitations are evident when MRI measurements are compared with MRI-derived computational models, as it will be shown later on. Due to these limitations, 4D Flow CMR is not yet extensively used in common practice as an alternative to conventional MRI or echocardiogram to assess blood flow, though many exploratory studies are being carried out, showing the great potential of the technique [53].

\subsection{Cardiovascular Computational Fluid Dynamics}

Computational Fluid Dynamics (CFD) consists in solving numerically the fluid dynamics governing equations in a specified geometry and with certain boundary con- 


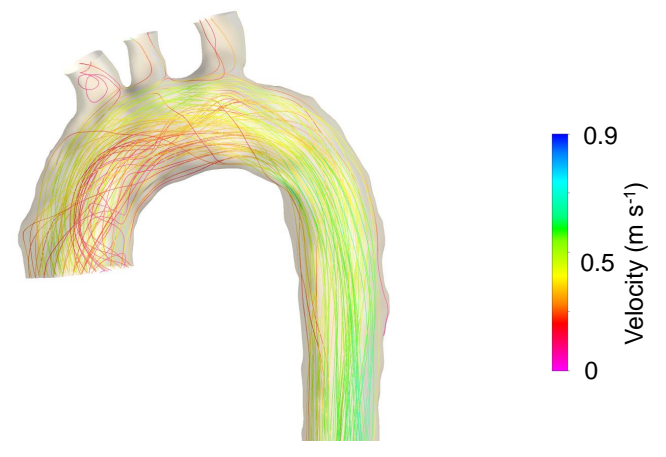

Figure 1.11: Visualization of the flow patterns of a patient specific CFD model of the aortic arch.

ditions. This approach provides arbitrarily high spatial and temporal resolution and the possibility of estimating all the quantities without recurring to averaging or interpolation, typical in experimental methods. In the figure 1.11 the blood flow in the aortic arch in late systole, computed by a CFD patient specific model, is shown using streamlines. In this chapter we will illustrate briefly the principles of fluid dynamics and the methods used in CFD.

\subsubsection{Fluid Dynamics}

"Fluid dynamics concerns itself with the study of the motion of fluids. The phenomena considered in fluid dynamics are macroscopic, therefore we will regard to fluid as continuous medium." [54]

This means that any small volume considered in the fluid is considered to be large enough that it still contains a great number of molecules. The study of fluid dynamics is based on three laws of conservation: the conservation of mass, momentum and energy. Under the assumption of incompressible flow and constant viscosity, using the conservation laws, we could write the the Navier-Stokes (NS) equations 3.1:

$$
\begin{array}{r}
\rho\left(\frac{\partial \bar{v}}{\partial t}+\bar{v} \cdot \nabla \bar{v}\right)=-\nabla p+\mu\left(\nabla^{2} \bar{v}\right)+\bar{f} \\
\nabla \cdot \bar{v}=0
\end{array}
$$

where $\bar{v}=(u, v, w)$ is the velocity vector, $u, v$ and $w$ are the velocity components in 
the $x, y$ and $z$ directions respectively, $\rho$ and $\mu$ are the density and the viscosity of the fluid respectively, $\bar{f}=\left(f_{x}, f_{y}, f_{z}\right)$ is the body force and $p$ is the pressure.

The NS equations are four non linear partial differential equations. There are few cases in which analytical solutions exist: numerical methods are needed in order to solve real-world problems. To do this, the NS equations have to be transformed into discrete algebraic equations. After defining the geometry of the system, its mesh, and the boundary conditions, these equations are solved in each mesh element associated with the fluid.

There are a number of methods used to discretise NS equations. The most used approaches are the finite volume and the finite difference methods [55]. In this research project, ANSYS CFX (version 18.0, ANSYS Inc. Cannonsburg PA, USA) is the software used to solve the computational fluid dynamic problems and it is based on the finite volume method.

In this approach, the fluid domain is divided in control volumes, and nodes are placed between control volume boundaries. The control volume could take any shape, therefore the mesh shape is more flexible. However this flexibility could lead to some errors if it is not adequately chosen. The principle of mass conservation is applied. Therefore the flux leaving a control volume is equal to the flux entering it.

Together with the geometry, also the boundary conditions of the geometry have to be defined. These features define where the flow is entering the system, where the flow goes out, the features of the boundaries, and the sets of fluid dynamic quantities defining the flow (e.g. velocities, pressures, flow rate). The choice of boundary conditions affect strongly the outcome of the simulation [56].

\subsubsection{Turbulence models}

In the laminar regime, flow follows the equations of continuity and momentum conservation. In this case, the inertia forces are smaller than the viscous forces, and disturbances are dissipated away granting the flow to be laminar. When the inertia forces grow, the disturbances are amplified and the flow regime starts getting turbulent [57].

In turbulent conditions the fluctuations of flow properties such as velocity, are 
completely random. If we consider a flow in turbulent regime, its velocity $\overline{u(t)}$ in a fixed point in space, could be expressed as $\overline{u(t)}=\bar{u}+\overline{u^{\prime}(t)}$, where $\bar{u}$ is the time average of the velocity, and $\overline{u^{\prime}(t)}$ is the fluctuating component. This description applies to all the flow quantities.

This suggests that in order to describe a turbulent system and therefore take into account flow quantities fluctuations, the governing equations 3.1 need to be modified in order to be resolved in a reasonable amount of computational time.

The average form of NS equations is called Reynolds Averaged Navier Stokes Equations (RANS). If we consider a 2D case, these equations would be similar to the NS equations, except for the presence of the terms $\overline{u^{\prime} u^{\prime}}, \overline{u^{\prime} v^{\prime}}$ and $\overline{v^{\prime} v^{\prime}}$, which will constitute three additional unknowns (in 3D we will have 9 additional unknowns). These terms are called Reynolds stresses.

There are different approaches in order to resolve these equations [58]. However this topic goes beyond the scope of this work.

\subsubsection{Wall Shear Stress}

Shear stress is the resistance of the fluid to the shear forces. As a consequence, a fluid at rest is not subject to shear stresses, whereas shear stresses are developed when the fluid is in motion, and the particles of the fluid have different velocities causing the fluid to deform.

For solids the shear stress $\tau$ is a function of the shear strain $\gamma$, while for the fluid is a function of the rate of shear strain $d \gamma / d t$. For Newtonian fluids the relation between shear stress and rate of strain is expressed by the Newton's Law of viscosity 1.15:

$$
\tau=\mu \frac{d \gamma}{d t}
$$

For an incompressible Newtonian fluid, to calculate the shear stress, the stress tensor $\sigma$ needs to be taken into account. In 3D cartesian coordinate $\sigma$ is given by 
Equation 1.3.1.2:

$$
\sigma=-\left(\begin{array}{ccc}
p & 0 & 0 \\
0 & p & 0 \\
0 & 0 & p
\end{array}\right)+\mu\left(\begin{array}{ccc}
2 \frac{\partial u}{\partial x} & \frac{\partial u}{\partial y}+\frac{\partial v}{\partial x} & \frac{\partial u}{\partial z}+\frac{\partial w}{\partial x} \\
\frac{\partial v}{\partial x}+\frac{\partial u}{\partial y} & 2 \frac{\partial v}{\partial y} & \frac{\partial v}{\partial z}+\frac{\partial w}{\partial y} \\
\frac{\partial w}{\partial x}+\frac{\partial u}{\partial z} & \frac{\partial w}{\partial y}+\frac{\partial v}{\partial z} & 2 \frac{\partial w}{\partial z}
\end{array}\right)
$$

To calculate the stress at a surface we need to compute $\sigma \cdot n$, which is a vector, and its viscous component in the tangential direction of the surface is the WSS. WSS is one of the most important markers in cardiovascular science [59], and in particular in atherosclerosis as it will be discussed in chapter 6 .

\subsubsection{Cardiovascular CFD}

In the last two decades CFD has been proven to be a powerful tools to study flow patterns in various anatomical regions and cardiovascular conditions such as the heart [31], intra-cranial aneurysms [11, 60], the thoracic aorta [61, 62, 33], and the carotid bifurcation $[43,63,64]$ in vitro or in vivo.

Indeed CFD predictions have so far proven to be reliable, showing good qualitative agreement with experimental techniques such as PC-MRI [63, 62, 33, 11].

Furthermore CFD models enable investigation of flow fields at a temporal and spatial resolution unachievable by any medical imaging methodology. Thanks to this, some quantities such as pressure [31, 43] and wall shear stress (WSS) [63, 60] maps are accessible in a detailed and non-invasive way.

Moreover CFD models are a vital resource also for device design, such as stents [65], in order to estimate the WSS perturbation induced by the stent itself, and heart valves [66], in order to have insight into their thrombogenic potential.

In clinical applications, CFD models are constructed following a standard pipeline, which could be summarized as follow:

1. geometry: a range of imaging technique could be used to provide the geom- 
etry of the system. Afterwards using reconstruction and segmentation techniques the medical images are converted into in silico geometric models.

2. mesh: in order to discretise the geometrical domain, a number of elements or cells are used to produce a mesh. The accuracy of the solution is conditioned by the refinement of the mesh. A good compromise enables to capture important features of the fluid dynamic and to have reasonable computational time.

3. boundary conditions: given that it is impossible to model the entire cardiovascular system, the region of interest needs an inlet and an outlet, and must be characterised from a physiological perspective. Quantities such as velocity, pressure and flow rate are usually used for this purpose. Together with them, the walls, enclosing the domain, and the fluid properties must be embedded in the model.

4. simulation: when the fluid and the domain properties are defined a CFD simulation could be launched.

5. results and validation: the data obtained from the simulation must be analysed and validated against literature or with experiments.

The CFD model prediction accuracy is determined by the model design and the input data. The resolution of medical devices is improving the quality of the experimental data, but the models' parametrisation of physiological quantities is still a challenge, since lots of assumptions and simplifications have to be done in order to have computationally feasible models.

\subsubsection{CFD MRI coupling}

Within the various imaging modality used for retrieving geometry and model parameters, MRI is getting more and more used in cardiovascular science because of its appealing features (non invasiveness, operator independence, flow sensitivity).

As described before PC MRI, and in particular 4D Flow CMR, produces two types of information: the signal intensity, that used for building the geometry of the 
anatomical area of interest, and the phase of the signal, that is used for retrieving the velocity field.

In order to retrieve the geometry the intensity of the signal has to be segmented. Afterwards the 3D model is obtained using image processing tools. For example when dealing with vessels, after segmenting the lumen, a surface (e.g. B-spline surface [41], least square cubic spline [64] ) is fitted to the sections to obtain a 3D model. Afterwards some smoothing algorithms are applied to the surface.

The vessel walls are usually modelled as rigid $[64,63,11,41,10,12]$, because in most cases the vessel compliance is within the MRI pixel dimension, or its a reasonable approximation considering the advantage from a computational cost perspective. However the wall properties must be chosen accordingly to the properties of the region of interest; for example aneurysmal blood vessels lack elastin and therefore the compliance is really small [11] and therefore the rigid approximation is reasonable. However in some anatomical regions, such as coronary arteries and cerebral aneurysm, it might be more appropriate to model the wall as compliant as this could make an impact on the fluid dynamic description $[67,68]$.

After meshing the surface, and modelling the wall of the region of interest, the inflow and outflow boundary conditions are set. In this case there are many different ways to approach the problem. These could be summarised in three main categories:

- uniform velocity profile: in this case the velocity profile is constant in the cross section for a given instant of time. In order to use this approach an extended length of the vessel is assumed in order to match the flow values measured in vivo [11, 56, 69];

- fully developed flow: a parabolic velocity profile is imposed at the inlet in order to match the measured flow-rate $[41,70]$;

- imaging fitted profile: the experimental velocity profile is used at the inlet, after being interpolated onto the mesh $[62,10,71]$.

There is no standard way to set the inflow condition, however the main fact is that 
MRI could not provide accurate flow rate measurements: in particular in order to use these flow rates as boundary condition for a transient model, they have to be correct at every time step. This incorrectness does not allow to use flow rate as outlet boundary condition [64], since it will not lead to mass conservation [12]. A common practice is to adjust them in order to do so [72].

The most common way for selecting the outlet boundary condition are zero pressure outlet or traction-free boundary condition $[63,10]$. However, when it comes to model blood flow in anatomical regions, zero pressure outlet models the situation in which the vessel is exposed to atmospheric pressure. This approach leads to inaccurate results, when considering compliant vessel or compressible fluid, since it ignores the changes of pressure and the flow rate by wave-reflection from the downstream vessels [73]. As a consequence in some works measured pressure is used as the outlet boundary condition $[69,56]$.

Generally in cardiovascular applications blood flow is assumed incompressible $[41,53,10,62,11]$. Even though it is well known that blood exhibits nonNewtonian behaviour, this feature mainly affects flow in small vessels and capillaries, and therefore Newtonian fluid model is generally assumed in large vessel [74].

\subsubsection{CFD and PC MRI: results comparison}

A good way of evaluating the outcome of MRI and MRI-derived CFD models is to compare their results. If we consider their outcome from a qualitative point of view, in the literature there is good qualitative agreement between the two techniques $[10,11,12,69]$, however there are substantial differences from a quantitative perspective.

Cebral et al. [10] assessed the performances of 4D Flow CMR and CFD through studying the haemodynamics in normal cerebral arteries. In these vessels, hemodynamics plays a central role in the understanding of the underlying mechanisms of initiation and progression of cerebrovascular diseases, e.g. strokes, in the majority of the case caused by the rupture of a cerebral aneurysm. In Figure 1.12 the flow patterns at systole in the cerebral arteries of a subject studied with MRI 


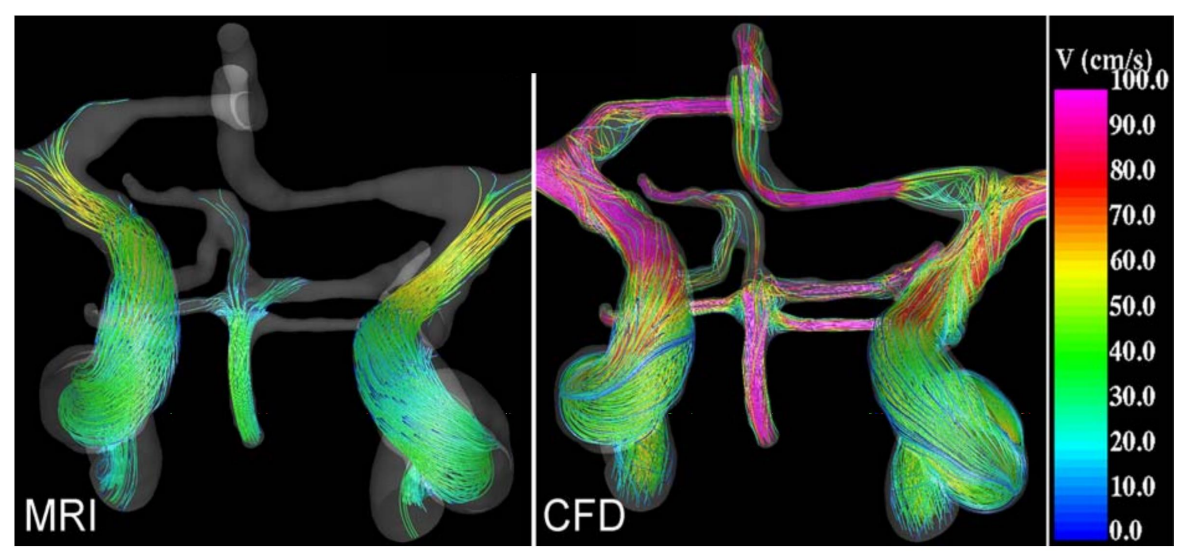

Figure 1.12: Blood-flow patterns at peak systole in the major cerebral arteries of a subject obtained by MRI data and CFD model (adapted from Cebral et al. [10]).

and CFD are shown. Qualitatively, the major flow structures, the swirling flows and the flow direction show a very good agreement. However from a quantitative perspective a number of differences arise: (1) the velocity magnitude is higher in CFD model with respect to MRI, (2) PC MRI is not capable of capturing accurately secondary flows (as a result MRI derived flow results are more laminar) and recirculation, which on the contrary are clearly visible in the CFD model, (3) the flow fields in PC-MRI are not divergence free.

In particular CFD models yield to a velocity that is from 5 to $50 \%$ higher with respect to PC MRI; this difference is larger as the arteries become smaller, as a consequence of the low signal and the limited resolution of the MRI. Contrarily CFD, thanks to the very fine mesh that can be used in areas characterized by strong velocity gradients, allows to capture more detailed features of the flow such as secondary flows or recirculation regions [41].

Another limitation of PC MRI arises when haemodynamic parameters such as WSS have to be estimated: in fact this would require interpolation or fitting of the measured flow field, which would likely lead to a poor estimation of this parameter which is essential for lots of applications.

In this context Boussel et al. [11] used CFD and flow sensitive MRI to study intracranial aneurysms, assessing among other fluid dynamic parameters, WSS. In Figure 1.13 the WSS maps in systole in the cerebral aneurysm of a subject studied 


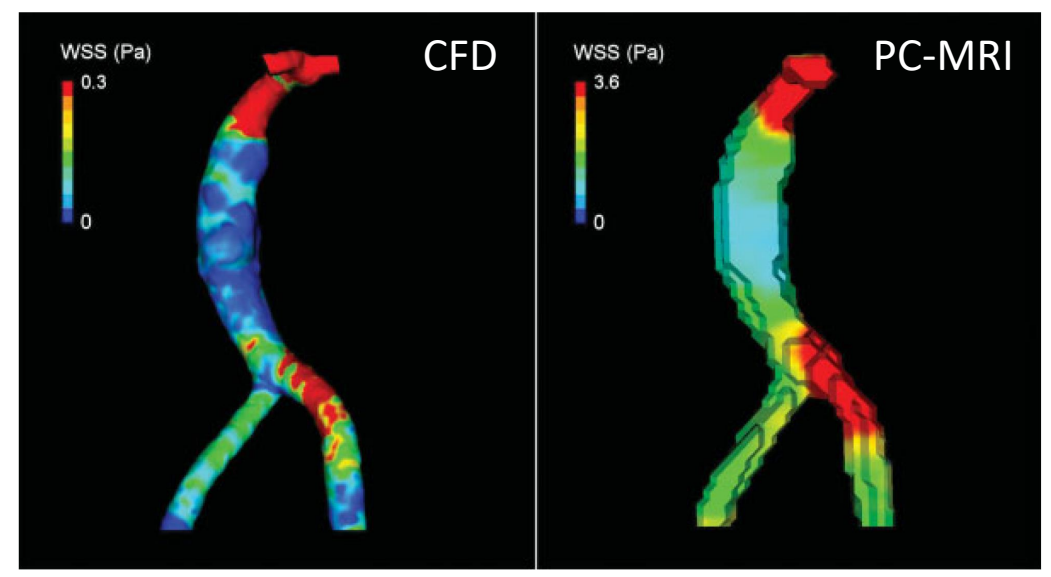

Figure 1.13: Wall shear stress (WSS) in a patient aneurysm in systolic phase computed with CFD (on the left) and PC-MRI (on the right) (adapted from Boussel et al. [11]).

with PC-MRI and CFD are shown. It is quite clear that even though there is a good visual agreement, from a quantitative perspective WSS calculated from MRI data results in an overestimation, particularly in area of high shear. As previously pointed out the main causes leading to these differences are the partial volume effect and the temporal averaging, characterizing MRI. Partial volume effect is predominant when considering flow in the proximity of the wall, since in these cases a single voxel might contain a broad distribution of velocities.

Differences in the haemodynamic descriptors between CFD and 4D Flow CMR were found also in larger vessels [12]. The purpose of Miyazaki et al. 2017 study, which was performed on a healthy adult and a child with a double-aortic arch, was to validate CFD using 4D Flow CMR. In Figure 1.14 the streamlines and the inplane velocity maps computed with a laminar CFD model and measured with 4D Flow CMR are shown for the healthy adult aorta. This study once again shows how the velocity distributions comparison highlights the discrepancies between the two techniques, from a quantitative as well as qualitative perspective. On the other hand CFD models are based on assumptions and impose simplifications which could impact the computed flow, and that could surely contribute to the differences between computational models and experimental results. 


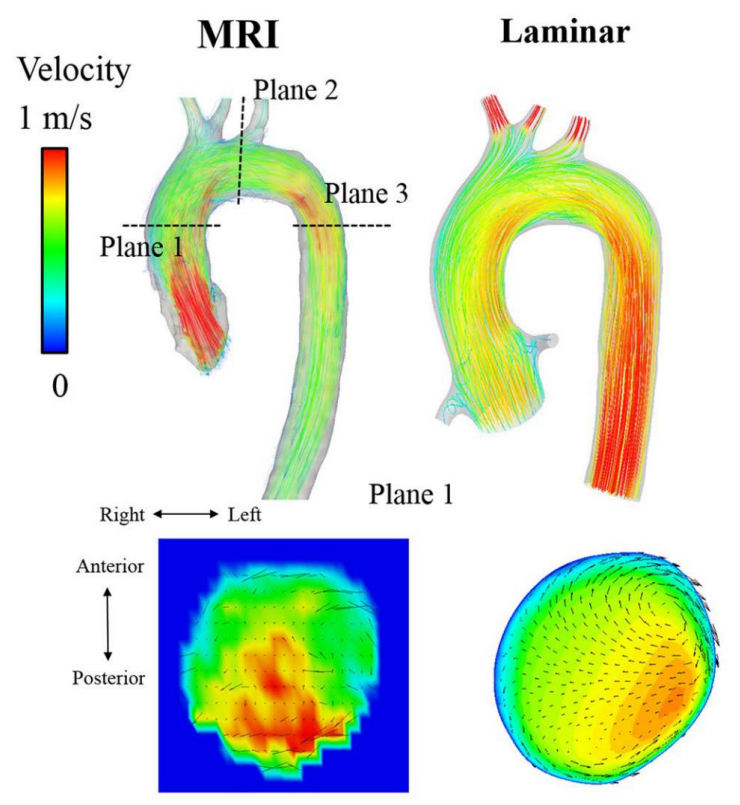

Figure 1.14: Streamlines and in-plane velocity distributions in one plane of the systolic peak in the healthy adult aorta measured with PC-MRI and Laminar CFD model (adapted from Miyazaki et al. [12]).

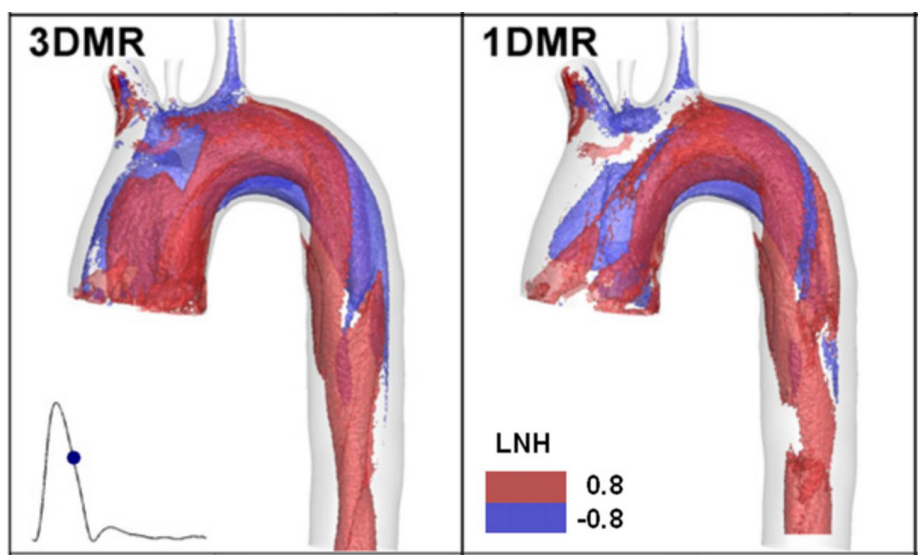

Figure 1.15: Localized Normalized Helicity at late systole in a patient specific aorta CFD model with full (3D) and axial (1D) blood velocity field prescribed as inlet boundary condition (adapted from Morbiducci et al. [13]).

For example Morbiducci et al. [13] highlight how the choice of the inlet boundary conditions can influence the entire numerical solution, showing in particular, the effect on the aorta haemodynamic. Figure 1.15 shows the impact on the CFD mod- 
els outcome when assuming as inlet boundary condition the full 3D velocity field and the axial one, when considering in the helicity of the flow. The parameter used (Localized Normalized Helicity LNH) shows the isosurfaces of aligned/opposed velocity and vorticity vector fields and its positive and negative values indicate counter-rotating flow structures. This study highlights how the incorporation of in vivo measured velocity profiles could be a necessary step in the pipeline for subjectspecific analysis of the aorta haemodynamics. It shows in fact how the assumption on the shape of the inlet velocity profile affects the topology of the bulk flow in the aorta, as a consequence of the flattening of the helical patterns in the flow when in-plane velocity components at the inlet are not taken into account.

Despite all its limitations, PC-MRI could provide realistic quantification of the main blood-flow structures [10] useful from a clinical perspective, but also to build computational model; CFD models on the other side could give access to high resolution flow fields, even though requiring necessary simplifications and assumptions that could impact the haemodynamic description.

A way to quantify the differences between the two methodologies is to have an high resolution reference field accessible experimentally.

\subsection{Particle Image Velocimetry}

\subsubsection{Principle}

Particle Image Velocimetry (PIV) is an in-vitro experimental technique that enables high resolution measurement of velocity fields. In order to understand the physics behind it, let us consider the experimental apparatus (Figure 1.16). As the name PIV implies, particles have to be added to the fluid in order to image the velocity field. In order for the seeding to do not affect the flow features, the particles have to be small enough to follow the flow. On the other hand they have to be big enough to scatter the light of the laser, used to illuminate the particles.

In order to have high frame rate PIV, high repetition rate diode-pumped lasers (e.g. Nd:YAG laser) need to be used. In order for the laser to penetrate the working fluid and the phantom without refraction, the fluid and the material of the phan- 


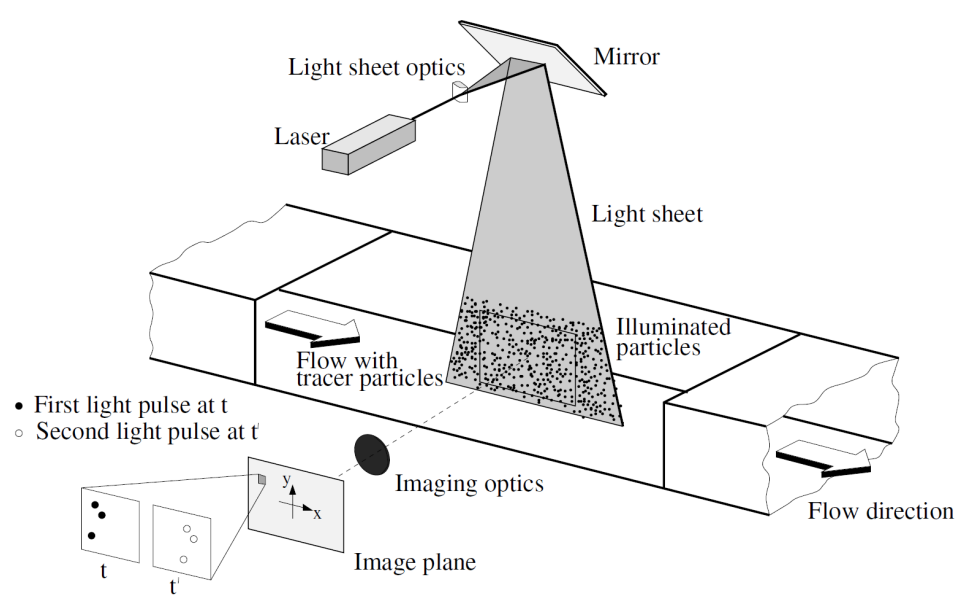

Figure 1.16: A 2D PIV experimental apparatus.

tom must have the same refractive index. This requirement strongly conditions the choice of the material of the phantom to be imaged as well as the working fluid.

The light scattered by the particles has to be recorded by means of an high quality camera/s. CMOS cameras operate at 1000 frames per second and they allow high-speed PIV (time resolution in the $\mu s$ ). The 2D PIV makes use of only one camera, allowing only in-plane velocity components measurements.

There are more complex PIV system, such as Stereoscopic and Tomographic PIV that make use of more cameras. In these cases however an accurate triangulation between the cameras used is needed [75]. Moreover the reconstruction process is quite demanding since multiple pairs of $2 \mathrm{D}$ images of the same instant of the cycle have to be processed in order to retrieve a 3D velocity map [76].

The velocity field is computed from the frames recorded, via post-processing. In particular, the field of view is divided in interrogation areas, and the displacement of the particles is determined for each interrogation area from the paired frames. The spatial resolution is related to this quantity. In fact it determines the number of independent velocity vectors that could be imaged, given the spatial resolution of the camera sensor.

In order to work, the laser and the camera need to be synchronized and triggered with the pump that is producing the flow. To study the flow in a precise instant of time of a pump cycle, in order to improve the SNR, multiple image pairs have to 
be recorded. This type of PIV is called phase resolved, and it is the one used in this study. Thus, a quantity that plays an important role is the trigger delay. This is the time delay between the start of the pump cycle and the instant of time in the pump cycle that we want to study.

Another crucial parameter is the time interval between the firing of the two laser pulses, that enables to record the images at time $t$ and $t^{\prime}$. This time delay must be long enough to allow the imaging of the displacement of the particles, and short enough to avoid particles leaving the interrogation plane between the subsequent illuminations.

Apart from the great spatial and temporal resolution, PIV presents other advantages [77]:

- Non-intrusive velocity measurements: since PIV is a purely optical technique, it does not employ any probes that could disturb the flow.

- Whole field technique: PIV allows to record image of large portion of the flow to be studied.

The main drawbacks of 2D PIV could be summarized in [78]:

- Particle choice: the seeding, as said before, could affect the properties of the the flow. For instance, if the density of the particles used is not the same as the fluid they will not follow the fluid. The same will happen if they are too big.

- Only 2D velocity components: the out of plane component of the velocity is not accessible.

- Averaged velocity field: in phase resolved PIV, the velocity field of an instant of time is the result of cross correlated image pairs. The cross correlation process leads to an average spatial representation of the velocity field.

- Homogeneous displacement assumption in PIV is assumed that the displacement of the particles, used for the seeding, is homogeneous in the interrogation areas. This assumption does not always apply and the correlation 
function is affected by out of plane velocity components and spacial velocity gradients.

- Mismatch of the refractive index: a slight mismatch in the refractive index between the model and the fluid could cause light refraction and optical distortion.

- Misalignment of the laser: if the laser sheet is not coplanar with the flow, the velocity field estimation is inaccurate.

\subsubsection{PIV in cardiovascular imaging as a validation tool}

The use of PIV in medical imaging has mainly the purpose of validation; the measured high resolution flow field is used as reference for computational models of aneurysms [79, 80], carotid arterys [78], aortic valves [81, 82], total cavopulmonary connection [14], or device implantation such us stents [83]. With the same purpose, PIV has been used also to validate MRI sequences [15, 84].

(a)

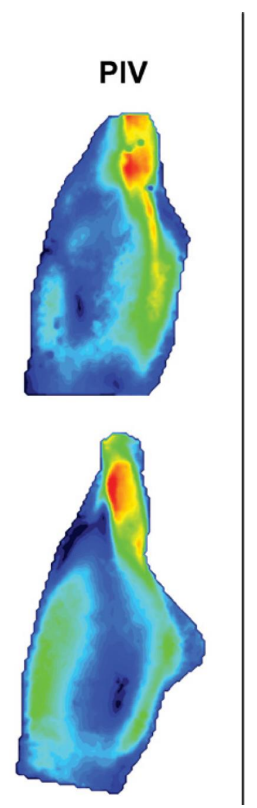

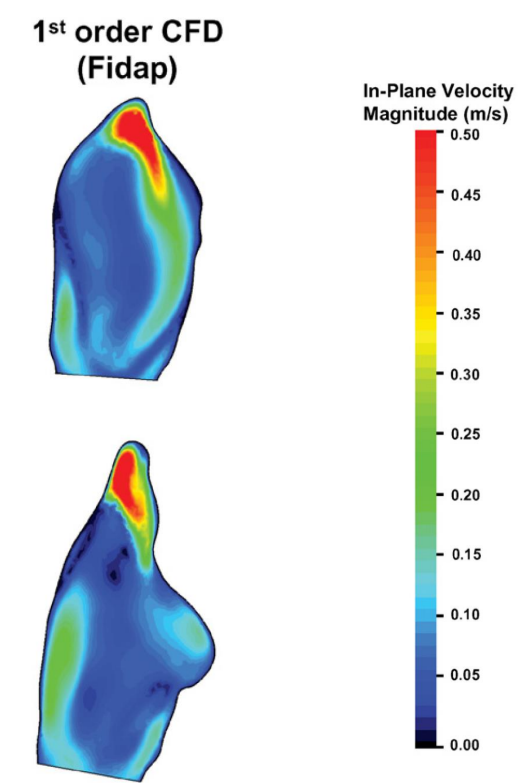

(b)

(a)

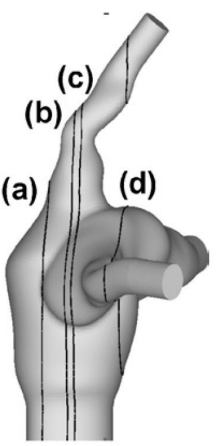

Figure 1.17: In-plane velocity estimated by a laminar CFD mode and measured with PIV in two planes of a TCPC model (adapted from Pekkan et al. [14]).

Raschi et al. [80] investigated haemodynamics in a model of cerebral aneurysm. Their objective was to investigate whether experimental and compu- 
tational models derived from computed tomography angiography of the aneurysm would lead to a consistent fluid dynamics description. This study showed how the CFD and PIV techniques yielded consistent flow patterns; nevertheless some difference in magnitude were observed. The main difference in the velocity magnitude was close to the wall where the PIV led to smaller velocities (Figure 1.17). This was likely due to the experimental error. In general, CFD yielded higher velocity magnitudes, and the source of this discrepancy was attributed to an imperfect match of the CFD model boundary conditions.

Pekkan et al. [14] used PIV to assess the prediction capability of a CFD model by studying the total cavo-pulmonary connection in steady flow condition. In this study CFD and PIV showed a remarkable agreement, especially considering the complexity of the anatomical region studied. The CFD velocities resulted however systematically lower $(\max 15 \%)$ with respect to the one measured by PIV. This discrepancy may be explained by the unpolished interior model surfaces that could have introduced a background noise effect.

A quantitative comparison of high resolution 3D PC-MRI, PIV and CFD was carried out by Van Ooji et al. [15] by assessing the flow of a real-size patientspecific phantom of an intra-cranial aneurysm. Also this study confirmed that good qualitative and quantitative agreement exists between PC-MRI measurements, CFD simulations and PIV measurements of flow patterns, on steady and pulsatile conditions. However the difference between CFD simulations and PC-MRI measurements $(\mathrm{RMSE}=4-5 \%)$ was smaller than that between PIV and PC-MRI measurements (10-12\%). In Figure 1.18 the velocity vectors computed with CFD and measured with MRI and PIV in a slice of the aneurysm in the steady experiment are shown. However these findings need to be interpreted taking into account a study limitations: a different fluid was used in the PIV experiment and in the PC-MRI measurement and a scaling of the results measured with PIV was then necessary, which may have introduced bias. Moreover in this study a PC-MRI sequence with a resolution of $0.2 \times 0.33 \times 0.2 \mathrm{~mm}^{3}$ was used; in clinical practice the resolution is at least 5 times lower, raising an issue on the fundamental question about the ability 


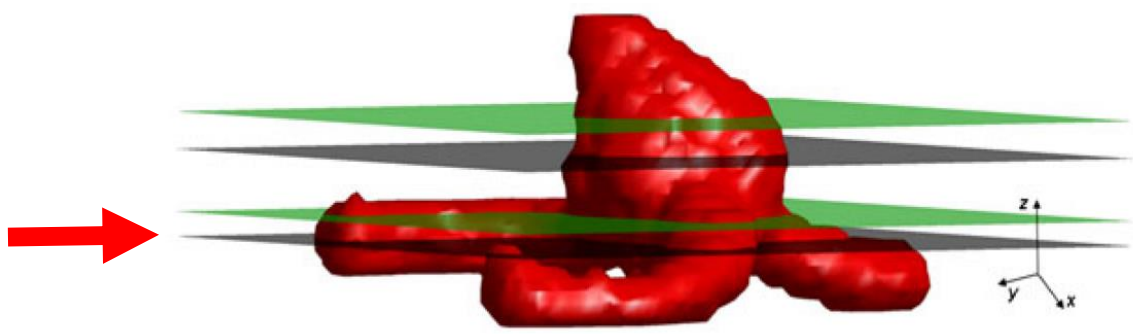

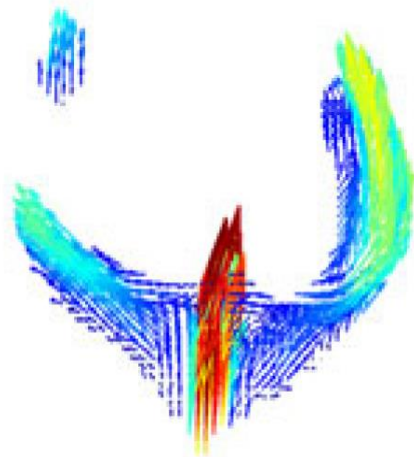

a. MRI

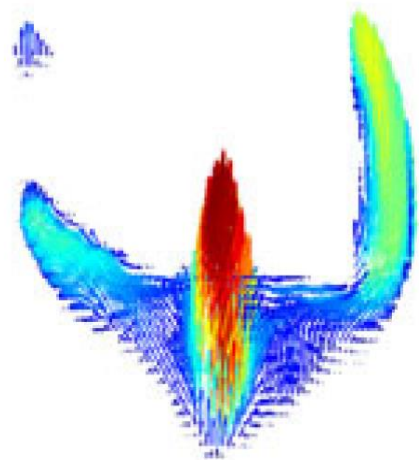

d. MRI

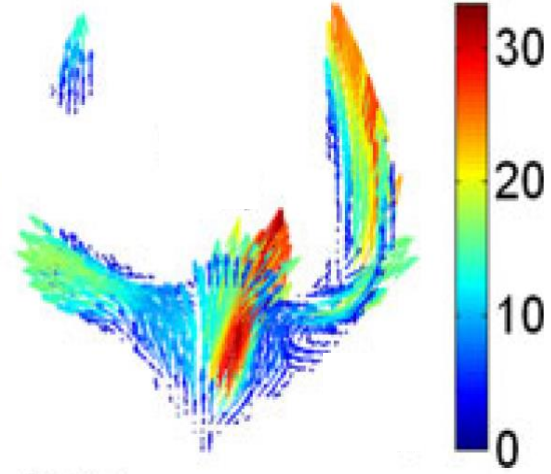

b. PIV

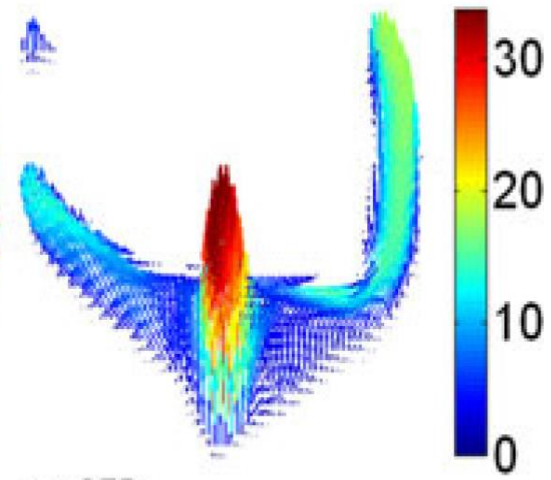

e. CFD

Figure 1.18: Velocity fields for steady measurement in the cerebral aneurysm. (a) MRI and (b ) particle image velocimetry (PIV) velocity vectors; (d) MRI and (e) computational fluid dynamics (CFD) in plane velocity vectors images in the slice indicated by the red arrow in the top row (adapted from Van Ooji et al. [15].

of the clinical used PC-MRI to correctly measure flow features in a reasonable scan time. 


\subsection{Data driven computational approaches}

The knowledge of 3D flow fields in the cardiovascular system, as mentioned, has provided new insights in the understanding of CVDs diagnosis and progression. Besides the velocity values and paths, also pressure changes in segments of the cardiovascular system is a critical parameter for diagnosis and treatment planning in many cardiovascular diseases, such as aortic valve stenosis [85] and aortic coarctation [86]. Flow fields measured with PC-MRI could be used to calculate pressure gradients. In particular Ebbers et al. [87] used the pressure Poisson equation using a multigrid-based solver, which works directly on the domain defined by the specific region of interest, obtaining very good results validated in silico, in vitro, and in vivo in the aorta.

A similar approach, based on finite elements, is the one suggested by Krittian et al. [88], that allows this method to be less sensitive to the boundary conditions. Moreover the proposed methodology allows to build an automatic mesh based on the MR data as well as a mesh with modified element size to interpolate the experimental data.

The limit of this approach lies in the decreasing accuracy when considering narrow flows and in the fact that the higher resolution is achieved via interpolation [89].

In a recent work, Marlevi et al. [90] suggested another interesting approach, exploiting imaging data to enable a more accurate estimate of pressure, by using a virtual work energy. This technique demonstrated the capability of estimating invivo relative pressure throughout the cardiac cycle, with an high accuracy and in the aortic coarctation scenario.

However the flaw of these methodologies is that they allow only to obtain the pressure estimate, and do not improve in any way the blood flow field computation. Moreover relying only on 4D Flow MRI data they carry the inaccuracy of the measured flow and therefore its limited resolution.

An approach that would allow to make use of the experimental data but at the same time rely on the accuracy of the CFD in reconstructing the flow was proposed 
by Szmyds et al. [91] in which computational methods and experimental techniques were merged together. In particular, their method allowed to pool sparse computational and experimental data to create a composite dataset and alleviate experimental uncertainties and theoretical constraints in the simulations. This approach was applied to thermofluid phenomena (e.g. convective heat transfer), but little is known about how the CFD and experimental data interact in this framework.

This concept - data assimilation - has become a common approach in atmospheric flow simulations for weather forecasting, where CFD results are 'corrected' by measured data at some sampling locations in the flow domain [92].

Hayase et al. [93] introduced this concept for the first time in biomedical fluid dynamic problems. The approach is based on the introduction of a body force in CFD which acts as a feedback term proportional to the difference between experimental and computational measurements, such that the experimentally-measured flow patterns are reflected in the computed ones, that strictly follow fluid dynamic principles.

These concepts have been applied to ultrasonic colour Doppler measurements of the aortic flow [94] and to 4D Flow CMR of a cerebral aneurysm [95], successfully leading to improved accuracy and resolution of the blood flow parameters which could provide a more accurate diagnosis. The approach has been tested on small regions of interest using 4D Flow CMR data, without consideration of errors in PC MRI measurement, nor a validation of the reconstructed flow field.

Recently, Rispoli et al. [96] used a similar approach to CFD simulations of the blood flow of the carotid bifurcation using PC MRI. In this study, however, the mesh had to be uniform and therefore did not ensure the flexibility required by typical cardiovascular problems, in which a refined mesh is necessary in proximity of the walls.

Therefore a more flexible approach that would allow an integration of CFD and 4D Flow CMR data is needed: in particular a method implemented in commercial software, that would allow (1) to improve the resolution of the 4D Flow CMR velocity field, (2) to compute other important cardiovascular parameters (e.g. WSS, 
pressure) (3) a user defined mesh. This would made the methodology applicable in a wider range of scenarios and would allow to compute all the markers of interest with high accuracy. Moreover a validation of this approach with an high resolution reference field would allow to understand how the coupling could be performed and the limitation in the current use of MRI data or computational model independently.

\subsection{Summary of the first chapter}

Let us briefly recapitulate the main points of the first chapter. In clinical practice, and in particular in cardiovascular diagnostics, 4D Flow CMR is a most insightful technique since it enables to measure blood flow and to retrieve CVDs biomarkers. MRI is non-invasive, it does not use ionizing radiation, it is comparatively operator independent, and enables to access flow characteristics. However due to its limited spatial and temporal resolution and the experimental noise, the parameters retrieved with this imaging modality are susceptible to errors. Another technique, widely used in engineering, is CFD. It allows to solve fluid dynamic equations in user defined geometries and boundary conditions; contrarily to 4D Flow CMR, it provides high spatial and temporal resolution. These advantages have made it suitable for biomedical applications, in particular in cardiovascular fluid dynamics, where it is used in combination with experimental imaging modalities, and in particular 4D Flow CMR. In this case both the geometry and the boundary conditions are retrieved from the 4D Flow CMR data and embedded in CFD models. 


\section{Chapter 2}

\section{Aim and Objectives}

The aim of this work is to integrate 4D Flow CMR and CFD using the interaction of their velocity fields over all the domains of interest, rather than using the first one only as a boundary conditions source. In order to do this, firstly a clear idea of the limitations of the two methodologies is needed and also the quality of this coupling has to be assessed. A third modality, the PIV, was then considered to fulfil the aforementioned objectives. This technique is characterized by high spatial and temporal resolutions and could be used as a reference velocity field.

The first objective of this work was then to prove the feasibility of the suggested methodology. In particular a numerical application was carried out in a 2D pipe in order to understand the behaviour of the parameters of EMRI, and the consequences of the introduction of an external force in the system from a fluid dynamic perspective. Afterwards the methodology was tested on a $2 \mathrm{D}$ portion of a patient specific descending aorta to test the feasibility of EMRI with real 4D Flow CMR data.

The second objective of the presented research was to create an experimental set-up that would allow to assess the performances of EMRI and highlight the limitation of CFD model and 4D Flow CMR. To do so the velocity field measured with 4D Flow CMR, and computed with CFD and EMRI, were compared to the one obtained by 2D PIV. As a consequence the experimental set-up had to be MRIcompatible (i.e. all the equipments used have to be non-magnetic, so that they could be placed safely inside an MRI imaging room) and at the same time PIV- 
compatible (i.e. the phantom to be imaged should have the same refractive index of the fluid used).

The third objective of this study was to show some applications of EMRI. Blood flow in a patient specific human aorta was studied, using a clinical 4D Flow CMR sequence. Finally EMRI was applied to study the WSS distribution in a carotid bifurcation phantom, and the flow-dependent temperature distribution in order to investigate temperature heterogeneities in athero-prone vessels. 


\section{Chapter 3}

\section{EMRI framework and preliminary}

\section{testing}

In this chapter the general framework of EMRI will be presented together with a numerical verification and a 2D application to 4D Flow CMR data. The results presented in this chapter have been published in the ASME Journal of Engineering and Science in Medical Diagnostics and Therapy [3].

\subsection{EMRI framework}

In this work, CFD is used in combination with 4D Flow CMR to enhance the resolution of the MRI and alleviate the modelling limitations of the CFD. We called our approach Enhanced magnetic resonance imaging (EMRI). The concept is applied, for the first time, to a body-fitted mesh and using a commercial CFD software.

The concept of EMRI (Figure 3.1) is CFD with correction of its velocities in reference to the velocity field acquired from 4D Flow CMR.

Therefore, the governing equations are the incompressible 3D Navier-Stokes equations:

$$
\begin{array}{r}
\rho\left(\frac{\partial \bar{v}}{\partial t}+\bar{v} \cdot \nabla \bar{v}\right)=-\nabla p+\mu\left(\nabla^{2} \bar{v}\right)+\bar{f} \\
\nabla \cdot \bar{v}=0
\end{array}
$$




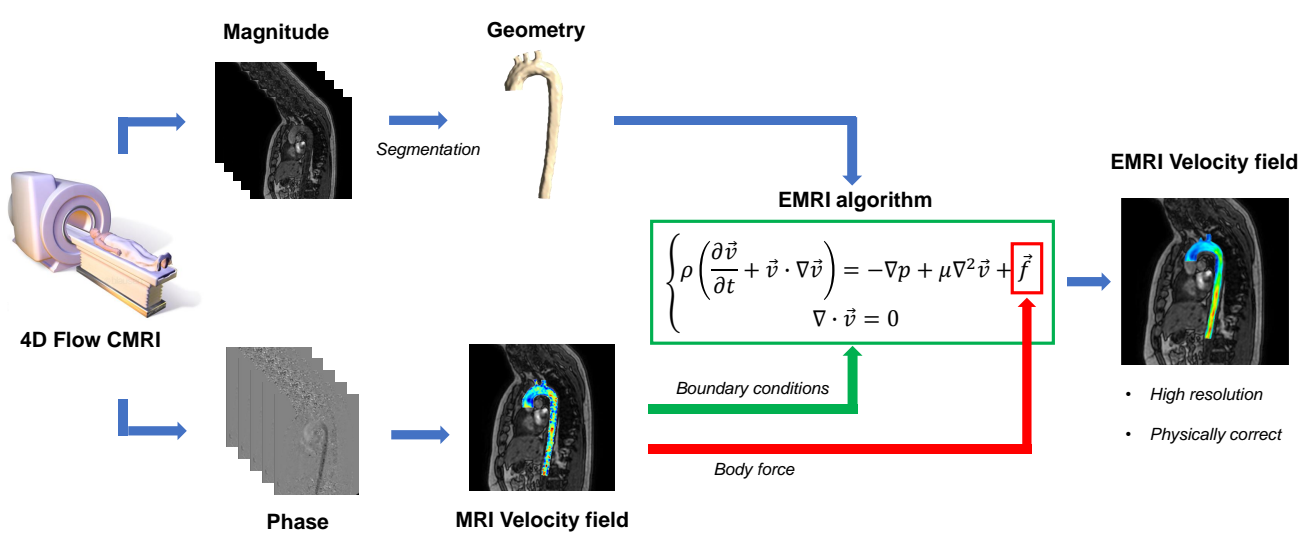

Figure 3.1: Diagram showing the EMRI workflow.

where $\vec{v}, \mathrm{p}, \rho$ and $\mu$ are the velocity, the pressure, the density and the viscosity of the fluid, respectively. The body force $\vec{f}$ is used to enable the incorporation of the experimentally-acquired velocity $\vec{v}_{\text {exp }}$ in CFD:

$$
\vec{f}=K \cdot\left(\vec{v}_{\exp }-\vec{v}\right)
$$

where, $K$ is the gain in this proportional external force and is expressed as follows:

$$
K=k\left(\frac{\rho U}{L}\right)
$$

where $k$ is a dimensionless constant, and $U$ and $L$ are the characteristic velocity and length of the system studied (chosen at the inlet).

The 'correction force' $\vec{f}$ is updated at every convergence iteration within one time step, allowing the velocity field computed to converge towards the experimental velocities.

If $k$ is kept constant across the computational domain and over a simulation, the strength of the force is varied only by the discrepancy between the computational and experimental velocity. Otherwise, $k$ would be the coefficient weighting the 
velocity difference.

The correction force becomes closer to zero when the weighted difference between the experimental velocities and the CFD derived ones reduces, and the fluid dynamic description of the measurement better approaches the theoretical solution of the Navier-Stokes Equations.

\subsubsection{Computational aspects}

In this study, steady state approximations were adopted, assuming that the flows in the different instants of the cardiac cycle can be computed independently.

The simulations were conducted using a commercial computational fluid dynamic solver ANSYS CFX. The governing equations were discretized in space using an element-based finite volume method, where volume and surface integrations are performed at the Gaussian integration points on each element/face using tri-linear shape function, interpolating nodal values of velocity and pressure in $3 \mathrm{D}$ within each element.

The linearised system by the spatial discretization was then solved with an algebraic multi-grid solver. The convergence was evaluated based on the root mean square (RMS) of the residuals of the governing equations. The convergence criterion was set to $10^{-6}$.

Computations were conducted using 5 cores on a standard desktop workstations (Intel Core i7 6700K 4GHz, 16GB RAM, 8 cores).

\subsection{Numerical verification}

In order to assess the feasibility and the accuracy of EMRI, a series of numerical experiments were designed. In particular the flow in a 2D channel (width $d x=0.0025$ $\mathrm{m}$ and length $\mathrm{dy}=0.030 \mathrm{~m}$ ) was studied (Figure 3.2), which has the advantage of having an analytical solution, the known parabolic velocity profile (equation 3.5):

$$
v_{y}=\frac{3}{2}\left(v_{\text {inlet }}\right)_{y}\left(1-\left(\frac{2 x}{d}\right)^{2}\right)
$$


where $\mathrm{d}$ is the width of the channel, $v_{y}$ is the velocity in the direction, and $v_{\text {inlet }}$ is the axial velocity at the inlet of the channel.

The main point of examination was the analysis of the behaviour of the development of flow along the channel. This would normally require a distance equal to the entry length if started from a flat velocity profile; in this case a fully-developed profile was enforced by the EMRI scheme, aiming to 'accelerate' the flow development. This approach will allow the understanding of the application of EMRI to 4D Flow CMR data, where the experimental data are enforced in the domain of interest with the intent of having a developed flow without extending the physical domain to have a fully-developed profile.

The theoretical velocity profile (equation 3.5) was sampled using different grids (Table 3.1) and these values, which for consistency will be indicated with $v_{\text {exp }}$, were then used by the EMRI scheme.

The fluid was modelled as blood, assuming it to be incompressible and Newtonian, with its density and viscosity set to $1060 \mathrm{~kg} \cdot \mathrm{m}^{-3}$ and $0.004 \mathrm{~Pa} \cdot \mathrm{s}$. The flow was assumed laminar.

At the inlet a flat velocity profile with $v_{\text {inlet }}=0.2 \mathrm{~m} \cdot \mathrm{s}^{-1}$ was imposed, whilst at the outlet constant pressure was used. The channel's walls were assumed rigid and no-slip boundary condition was applied.

The entry length is given by (equation 3.6):

$$
L=0.05 \frac{\rho v_{y} d^{2}}{\mu}=0.01655 m
$$

The sensitivity of the computational outcome of EMRI for the application of this method in real scenarios, was assessed by studying the following parameters:

1. number of sampling data points across the channel width at which $v_{\exp }$ is given;

2. $\mathrm{k}$, the constant in the body force.

3. enhancement in presence of noise; 
4. temporal persistence of the enhancement;

A mesh convergence test was performed (see Appendix A.1) to select the most suitable discretization, considering the value of velocity on the longitudinal axis of the channel, and considering 1\% change as threshold. However given the objective of this study, the mesh chosen was the most refined one, characterized by 72717 quad elements (Figure 3.2).

a)

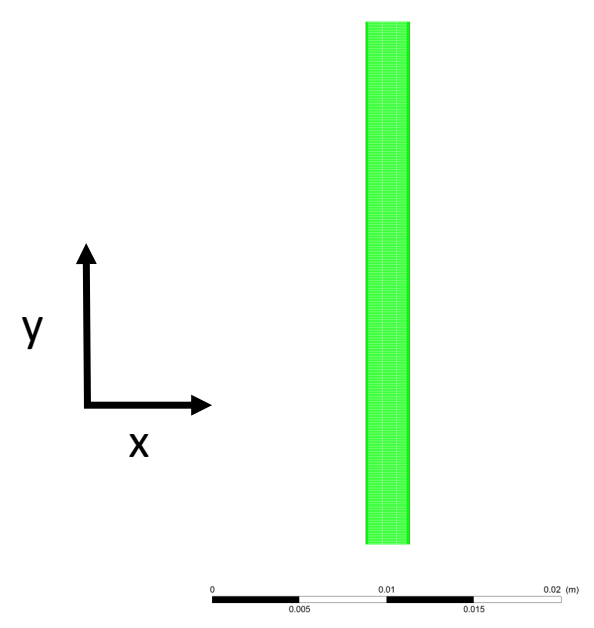

b)

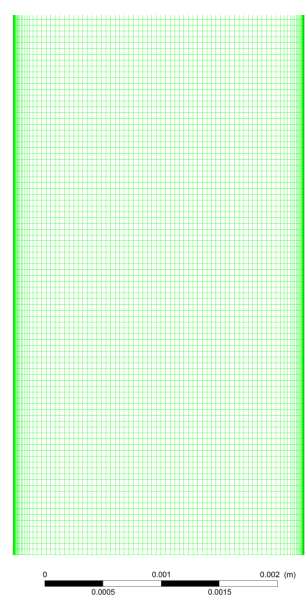

Figure 3.2: Quad mesh of the 2D channel.

\subsubsection{Enhancement with different sampling grids}

In the first test the computational method was performed using 4 different grids of sampling points (Table 3.1) where $\overrightarrow{v_{\exp }}$ was given by Equation 3.5 at a uniform spatial interval. The velocities computed using the EMRI scheme via the correction force $\vec{f}$ with $\mathrm{k}=1$, were compared at $\mathrm{y}=0.003 \mathrm{~m}$ (non-developed flow region), together with the kinetic energy of the system, evaluated as (equation 3.7):

$$
E_{k i n}=\rho \frac{v^{2}}{2}
$$

$E_{k i n}$ was selected as quantity because it is a scalar and convenient descriptor of the total correction the EMRI is performing via experimental data enforcement. 
Table 3.1: Energy and energy change (\%) for the different grids at which the experimental velocity $v_{\exp }$ was sampled

\begin{tabular}{|c|c|c|c|}
\hline \hline Grid $n^{o}$ & Spacing (mm) & $\begin{array}{c}\text { Points along the } \\
\text { cross section }\end{array}$ & $\begin{array}{c}\text { Points along the } \\
\text { longitudinal } \\
\text { direction }\end{array}$ \\
\hline 1 & 0.5 & 6 & 61 \\
2 & 0.25 & 11 & 121 \\
3 & 0.1 & 26 & 301 \\
4 & 0.05 & 51 & 601 \\
\hline
\end{tabular}

Its value is proportional to the difference between $\vec{v}$ and $\overrightarrow{v_{\exp }}$ when convergence is reached. In the ideal case scenario this has to be minimal but when dealing with real data this is an inevitable price to pay for imposing 4D Flow CMR velocities in CFD.

Another indicator of the effect of the integration of the force in the system is the pressure drop along the longitudinal axis of the channel. This quantity was computed together with the force residuals, given by the magnitude of the external force term at the last iteration of the simulation.

\subsubsection{Enhancement with different force parameter $k$}

The effect of the force parameter k, was assessed in the case of high resolution grid (grid $n^{o} 4$ ), which provides the best depiction of the velocity profile, by using different values of $\mathrm{k}$, in particular $0,10^{-1}, 1,10,10^{2}$ and $10^{3}$, and assess how the strength of the enforcements affects the EMRI computation of the flow. The outcomes of the simulations were compared in terms of the velocity difference between $\overrightarrow{v_{e x p}}$ and the computed velocity, the energy of the system and the number of convergence iterations.

\subsubsection{Enhancement in presence of noise}

In order to translate the EMRI approach to a real case scenario, the enhancement needs to be verified in presence of noisy data. 4D Flow CMR data without contrast agent have an signal-to-noise ratio (SNR) around 30 [97], expressed as (equation 3.8): 


$$
S N R=\frac{\bar{S}}{\sigma_{N}}
$$

where $\bar{S}$ is the average signal in the region of flow, and $\sigma_{N}$ is the standard deviation of the signal in a background area where there is no flow (i.e. noise). The effect of the enforcement was assessed in the case of high resolution grid (grid $n^{o}$ 4) with the most refined mesh, with $k=1$ using two values of SNR, 20 and 30 $\mathrm{dB}\left(S N R=10 \cdot \log _{10} S N R\right)$. The outcome of this test was evaluated in terms of the velocity (computed in the developed flow region $\mathrm{y}=0.025 \mathrm{~m}$ ) and the pressure (along the axis of the channel) with respect to the ones obtained in a absence of noise.

\subsubsection{Temporal persistence of the enhancement}

A time resolved simulation was run, with a steady velocity at the inlet, and with the application of the enforcement for a $\Delta t$, to assess the persistence of the enforcement in time. The total time was set at $0.5 \mathrm{~s}$ and the force was applied for $\Delta t=0.1 \mathrm{~s}$ from $t_{1}=0.1 s$ to $t_{2}=0.2 s$. The effect of the enforcement was assessed in the case of high resolution grid (grid $n^{o} 4$ ) with the most refined mesh. The outcome of this test was evaluated in terms of the velocity difference between $\overrightarrow{\exp }$ and the computed velocity, focusing on the time-steps following the removal of the external force.

\subsubsection{Results and discussion}

\subsubsection{Enhancement with different sampling grids}

In Figure 3.3 the velocity profiles at $\mathrm{y}=0.003 \mathrm{~m}$-approximately $20 \%$ into the entry length from the inlet - are shown for the simulations with and without correction force. The "measured velocities" $v_{\text {exp }}$, to be expected in the fully-developed region (equation 3.5), were given at the points shown in the (Figure 3.3).

The EMRI scheme forces the velocity profiles to approximate the parabolic profile. The computed velocity profiles show minor dependence to the resolution of the grids where the $\overrightarrow{\exp }$ data were given. This is obvious if one looks at the squared velocity difference between the parabolic and computed profiles and the relative root mean squared error (RMS) at different sampling points (Figure 3.3 e and f). 

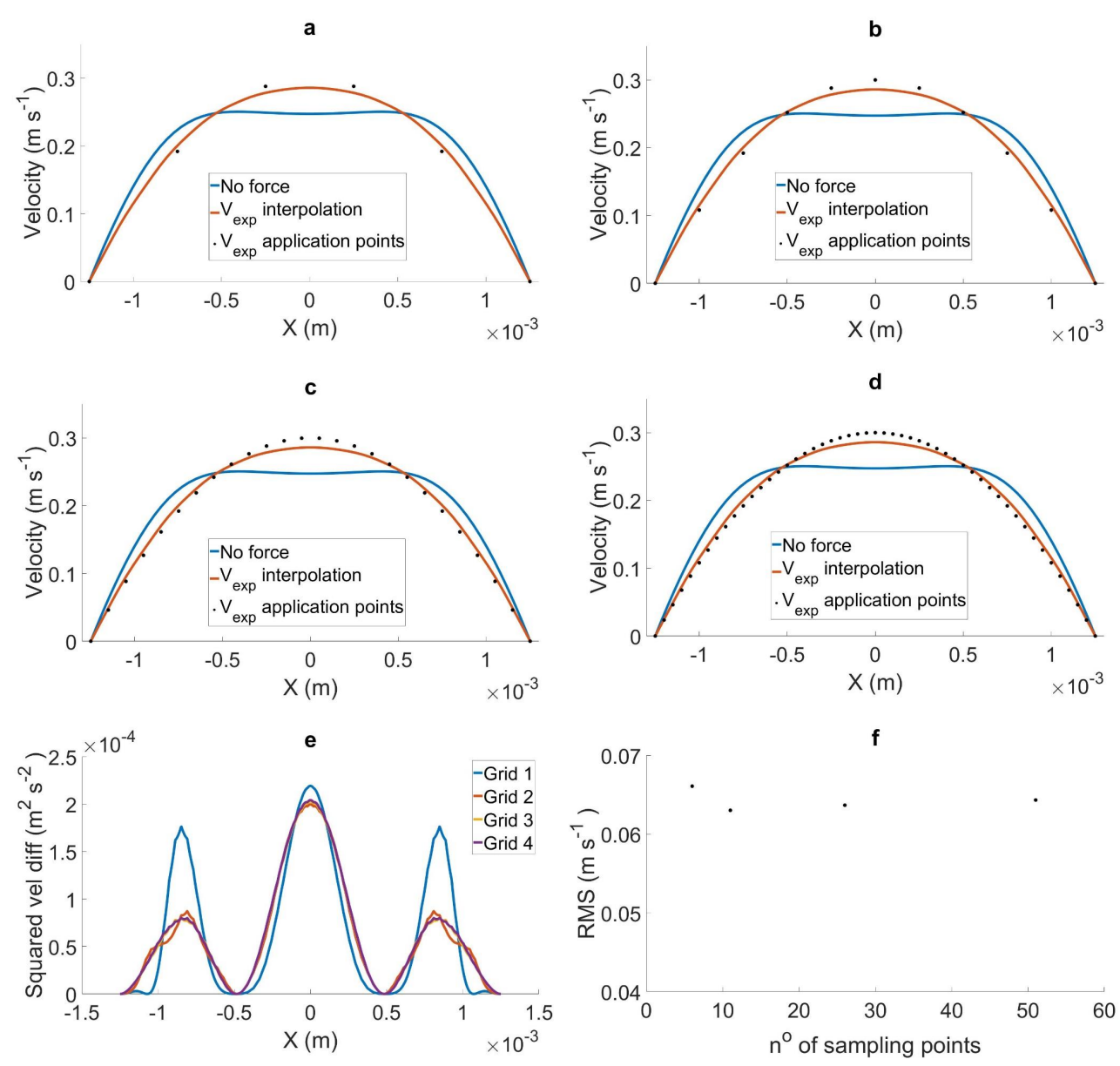

Figure 3.3: a), b), c), d) Velocity profiles at $y=0.003 \mathrm{~m}$ computed with different grids at which the experimental velocity $\mathrm{v}_{\exp }$ was sampled; the lines ' $\mathrm{v}_{\exp }$ interpolation' indicate the interpolation of the experimental points on the mesh and the "no force' lines are the velocity profile in the case without external force e) squared velocity difference between the parabolic profile and the computed ones with different grids of sampling points and f) root mean squared difference.

The highest RMS is obtained for the case with the lowest sampling points, and in all the other cases the RMS is of the same magnitude (Figure $3.4 \mathrm{f}$ ), showing that 11 data points across the channel are sufficient to reflect the experimentally observed velocity profile.

Furthermore, by plotting the velocity along the longitudinal axis of the channel (from the inlet to the entry length) for the different density of sampling points (Figure $3.4 \mathrm{a}$ ), it is easy to highlight the effectiveness of the force in allowing a faster 
development of the flow profile.
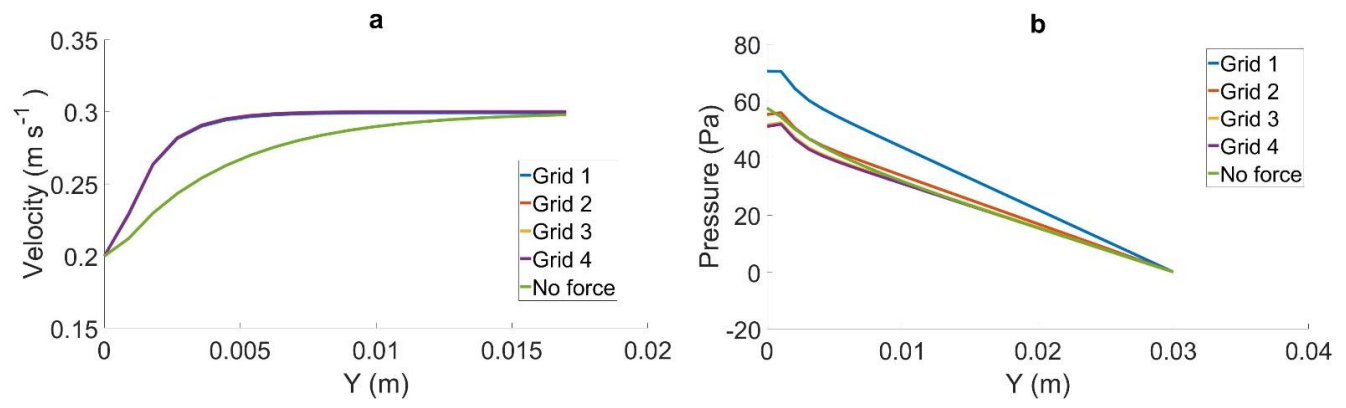

Figure 3.4: Velocity (a) and pressure (b) along the axis of the channel for the different grids at which the experimental velocity $\overrightarrow{v_{\exp }}$ was sampled, and with the case without enforcement.

It must be emphasized that the imposed force drives the flow to take a theoretically incorrect velocity profile since in the studied area the flow is not fully developed. This is visualized with the force residuals (force value at the last iteration) maps in Figure 3.5.

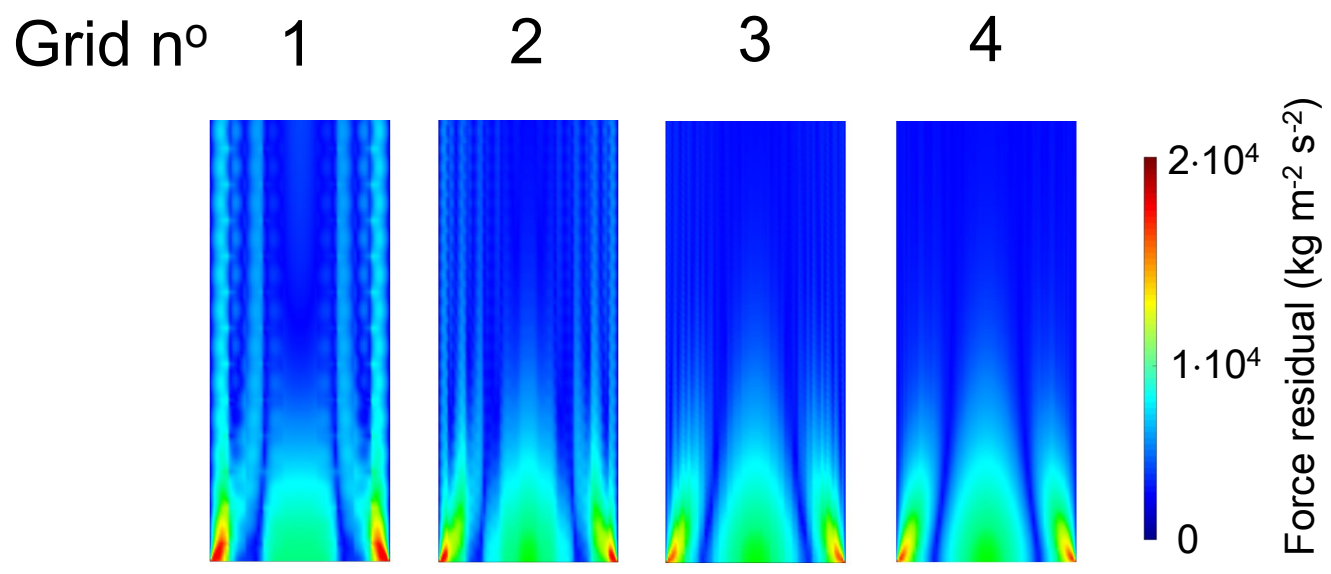

Figure 3.5: Force residuals maps in a portion of the 2D surface computed for the different grids at which the experimental velocity $v_{e} x p$ was sampled.

The residual maps show that an increase of the number of $v_{\exp }$ sampling points leads to a decrease of the residuals. Moreover the area where the residual is focused 
Table 3.2: Energy and energy change (\%) for the different grids at which the experimental velocity $\overrightarrow{v_{\text {exp }}}$ was sampled

\begin{tabular}{|c|c|c|}
\hline \hline Grid $n^{o}$ & $\begin{array}{c}\text { Energy of the } \\
\text { system }\left(\mathrm{Jkg}^{-1}\right)\end{array}$ & $\begin{array}{c}\text { Increase in the } \\
\text { energy (\%) }\end{array}$ \\
\hline 1 & 1834.1 & 1.431 \\
2 & 1828 & 1.096 \\
3 & 1827.3 & 1.055 \\
4 & 1824.25 & 1.053 \\
No force & 1808.2 & 0 \\
\hline
\end{tabular}

is the one around the inlet, where the discrepancy between computed velocity and $\overrightarrow{v_{\text {exp }}}$ is larger.

The effect of force residual could be described also in terms of kinetic energy (Table 3.2).

It is noticeable how a larger number of $\overrightarrow{\exp }$ sampling points leads to a smaller injection of kinetic energy in the system. This is due to the fact that the solution converged to a more physically-reasonable velocity profiles. Even in the worst case (grid $n^{o} 1$ ), the energy increase is only $1.5 \%$.

The increase of the residual amount in the inlet region is a consequence of the energy increase needed to accelerate the onset of the developed profile. This is reflected in the pressure drop along the channel (Figure $3.9 \mathrm{~b}$ ).

The velocity profiles in the developed flow region $(\mathrm{y}=0.025 \mathrm{~m})$ with different sampling point densities, shown in Figure 3.6, are closer to each other compared to those in the region of flow development. The only profile that differs from all the others is the one of the lowest sampling density (6 points across the channel).

The percentage differences of velocities are also shown in the figure, showing that when there is a sufficient number of sampling points the quantitative discrepancy with the parabolic distribution is small (less than $4 \%$ for more than 11 sampling points across the vessel cross section).

\subsubsection{Enhancement with different force parameter $\mathbf{k}$}

Figures 3.7 and 3.8 illustrate the results of the sensitivity test to another important parameter, $k$. The trends demonstrate that larger values of $\mathrm{k}$ lead to a stronger en- 
forcement of the parabolic profile, even in the area where the flow is still developing.
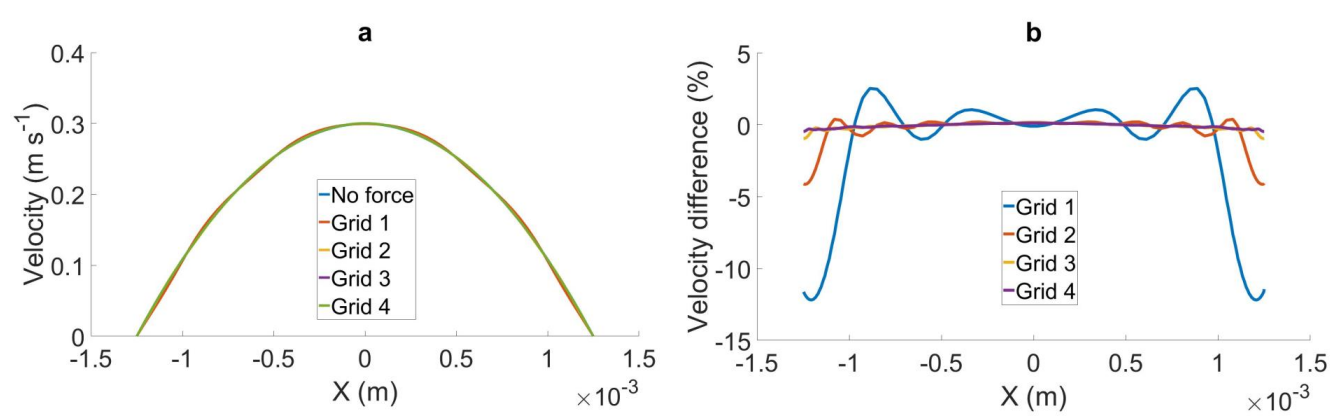

Figure 3.6: a) Velocity profiles at $y=0.025 \mathrm{~m}$, calculated for the different grids at which the experimental velocity $\mathrm{v}$ was given. b) Percentage difference between the velocity profiles with various sampling point densities at $y=0.025 \mathrm{~m}$ and the case with no force

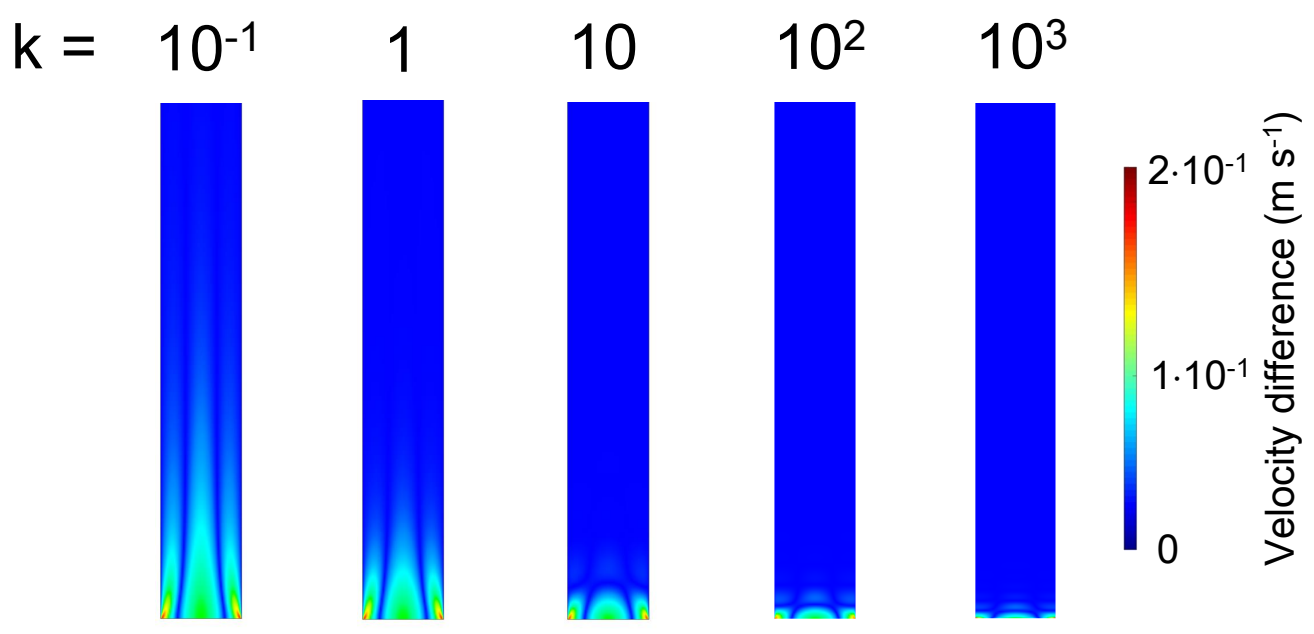

Figure 3.7: Velocity difference maps in a portion of the 2D surface computed at different values of $\mathrm{k}$

Higher degree of velocity profile enforcement means that a higher level of external energy is injected into the system, as shown in Figure 3.8 a. However, the increase it is only of $2.1 \%$ for $\mathrm{k}=10^{3}$, as shown quantitatively in Table 3.3.

Observing the behaviour of the number of convergence iterations (Figure $3.8 \mathrm{~b}$ and Table 3.3), it is clear how markedly it is influenced by $k$, resulting in an almost halving of iterations from $k=10$ to $k=10^{2}$. Difficulties in convergence through 
Table 3.3: Energy and duration of the simulation change (\%) at different values of $\mathrm{k}$

\begin{tabular}{|c|c|c|}
\hline \hline $\mathbf{k}$ & $\begin{array}{c}\text { Energy increase } \\
(\boldsymbol{\%})\end{array}$ & $\begin{array}{c}n^{o} \text { iterations } \\
\text { variation }(\boldsymbol{\%})\end{array}$ \\
\hline $10^{-1}$ & 0.22 & -7.6 \\
1 & 0.98 & -43 \\
10 & 1.60 & -43 \\
$10^{2}$ & 1.82 & -26 \\
$10^{3}$ & 2.14 & +213.9 \\
\hline \hline
\end{tabular}

the iterative solution process arise when the enforcement is too strong, and as a consequence the duration of the simulation increased more than two-folds for the largest level of $k=10^{3}$.

A similar trend in the behaviour associated with the amount of enforcement was reported by Funamoto et al. [95], who observed a monotonic increase in the convergence toward the values enforced, when increasing the coefficient of the force (the equivalent of the $k$ used in this work), until divergence was observed.

This trend suggests that convergence is supported by the force term for a certain range of $k$, whereas when $k$ is above a certain threshold the force has an opposite effect. For large values of $k$, in fact, even small differences between $\vec{v}$ and $\overrightarrow{v_{\text {exp }}}$ play a major role and might create convergence problems. However, it should be considered that, in this scenario, the force is based on a $\overrightarrow{\exp }$ that is not affected by noise. Therefore, no matter how high $k$ is, the force would converge to zero in the developed flow area.

In real case scenario, where $\overrightarrow{e_{\text {exp }}}$ is extracted from 4D Flow CMR, the conver-

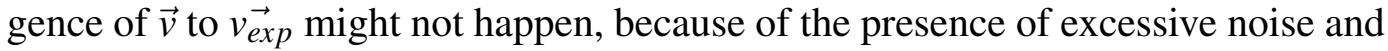
experimental errors. Therefore, the choice of $k$ requires careful considerations on the fluid dynamic of the system.

Also the pressure drop along the channel (Figure 3.9), reflected this trend, showing steady increases with increasing $k$, reaching very large values for $k=10^{3}$. The case examined constituted an extreme situation, where a flat velocity profile was imposed at the inlet and a parabolic profile was imposed immediately downstream, ending up with local high pressure gradient. 


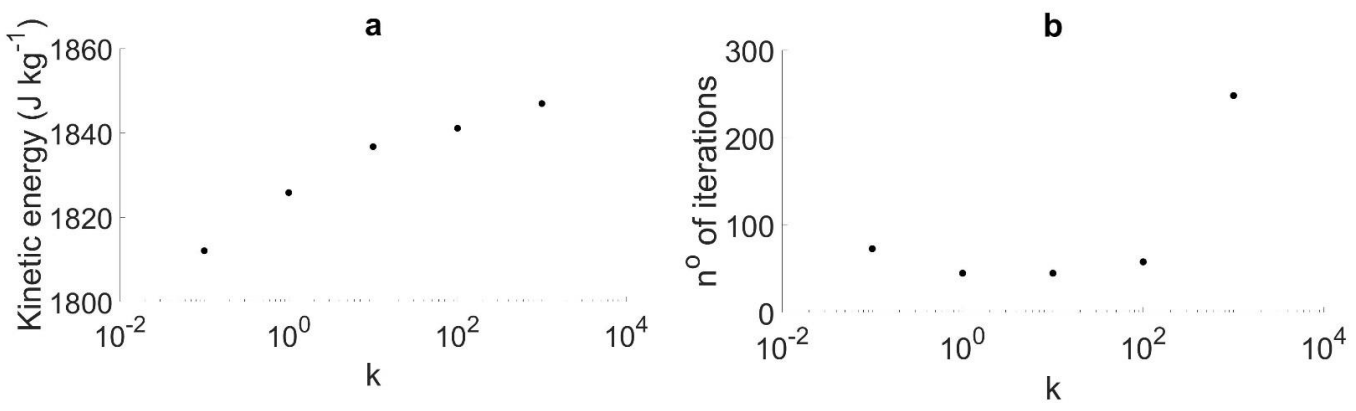

Figure 3.8: Kinetic energy of the system (a) and number of convergence iterations at different values of $\mathrm{k}$.

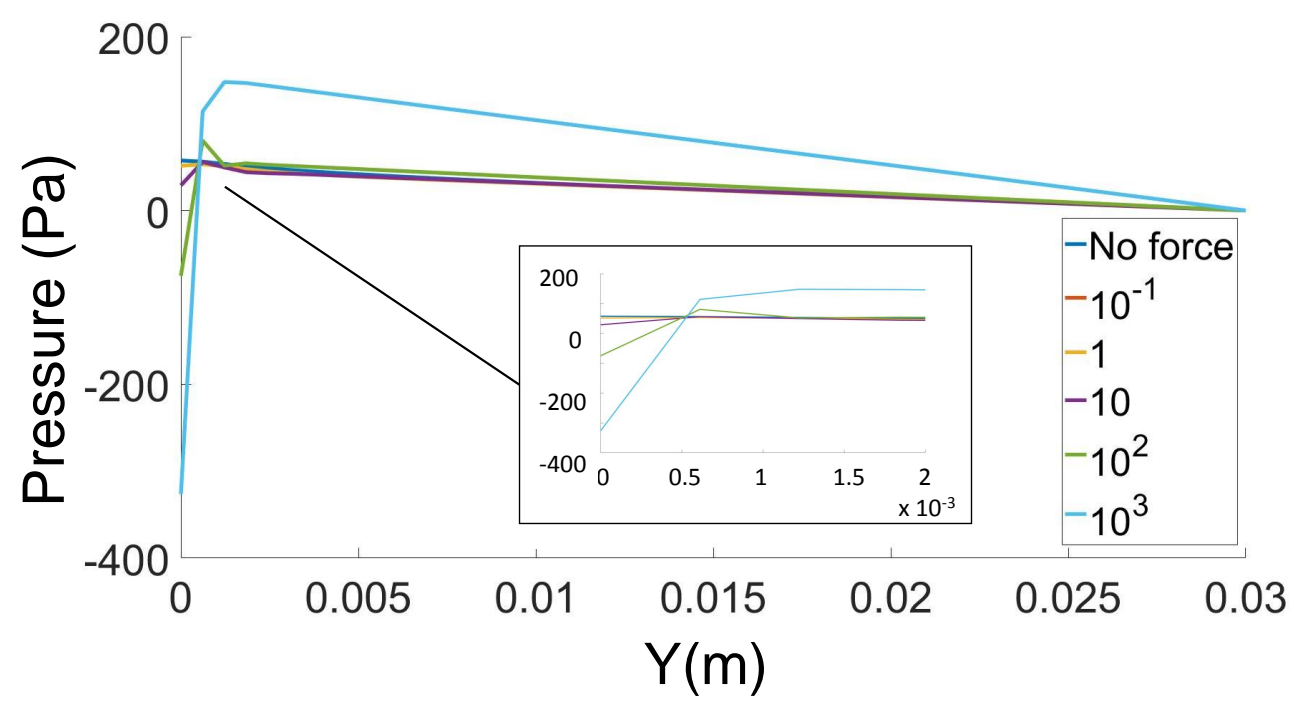

Figure 3.9: Pressure drop along the axis of the channel at different values of $k$ and without force.

\subsubsection{Enhancement in presence of noise}

In Figure $3.10 \mathrm{a}$ and b, the velocity profiles obtained 'experimentally' and computed with EMRI in the developed flow area $(y=0.025 \mathrm{~m})$ in the case with SNR 20 and $30 \mathrm{~dB}$ are shown respectively. In both cases, the EMRI 'corrects' for the noisy data and produces a velocity profile very close to the theoretical one. In order to have a quantitative assessment of the EMRI performances in this scenario, the velocity profiles in the developed flow area $(\mathrm{y}=0.025 \mathrm{~m})$ computed with EMRI in the case of SNR 20 and $30 \mathrm{~dB}$ are compared against the one obtained in absence of 
a

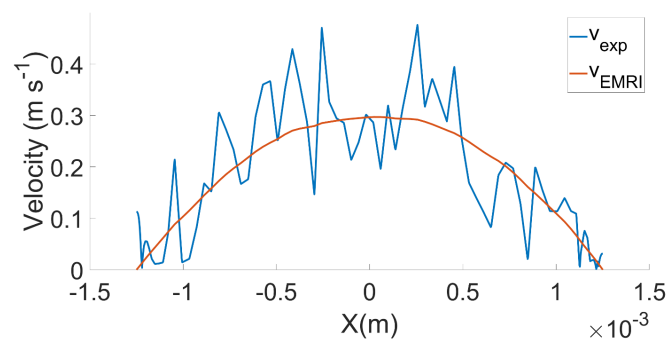

b

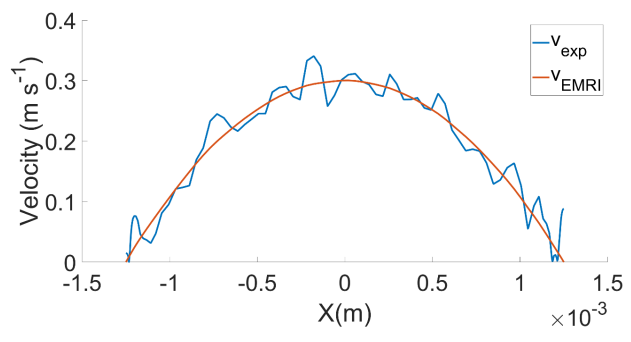

Figure 3.10: Velocity profiles obtained 'experimentally' and computed with EMRI in the developed flow area $(y=0.025 \mathrm{~m})$ in the case with SNR 20 (a) and $30 \mathrm{~dB}(\mathrm{~b})$.

noise (Figure 3.11 a). Moreover in Figure $3.11 \mathrm{~b}$ the differences between the EMRI computed velocity in the SNR 20 and $30 \mathrm{~dB}$ cases and the case without noise are plotted. The highest discrepancy between the velocity in the case with and without noise is of $1 \%$ and $2.5 \%$ for the SNR of $30 \mathrm{~dB}$ and $20 \mathrm{~dB}$ respectively.

a

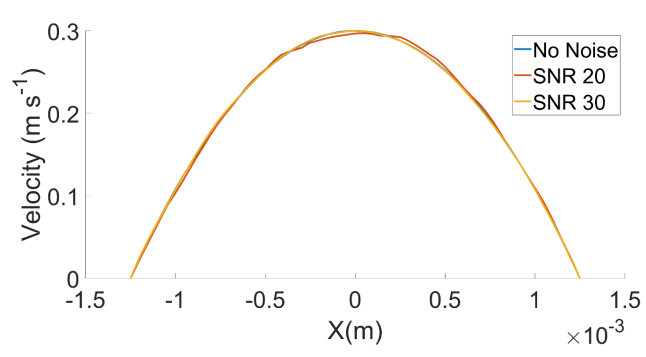

b

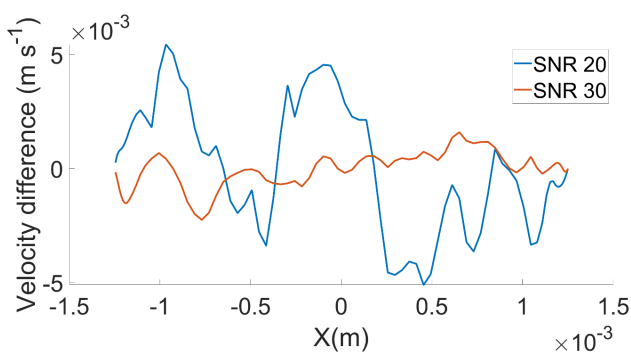

Figure 3.11: (a) Velocity profiles in the developed flow area $(y=0.025 \mathrm{~m})$ computed with EMRI in the case without noise, and with SNR 20 and $30 \mathrm{~dB}$; (b) velocity difference between the velocity computed with EMRI with noise and without noise for the two values of SNR.

The impact of the noise on the pressure estimate, is assessed by comparing the case with and without noise (Figure 3.12 a). In the case with SNR of $30 \mathrm{~dB}$ the discrepancy is of the order of $0.2 \mathrm{~Pa}$ whereas in the case with SNR of $20 \mathrm{~dB}$ is 3.2 $\mathrm{Pa}$ (Figure $3.12 \mathrm{~b}$ ) which account respectively for $0.5 \%$ and $1 \%$.

The results obtained for velocity and pressure demonstrate that EMRI is resilient to a noise of the order of the one that is typically observed in 4D Flow CMR data, and even in a case with a much lower SNR. Therefore the translation to real case scenario is feasible and would bring benefit to the estimate of velocity fields and pressures. 
a

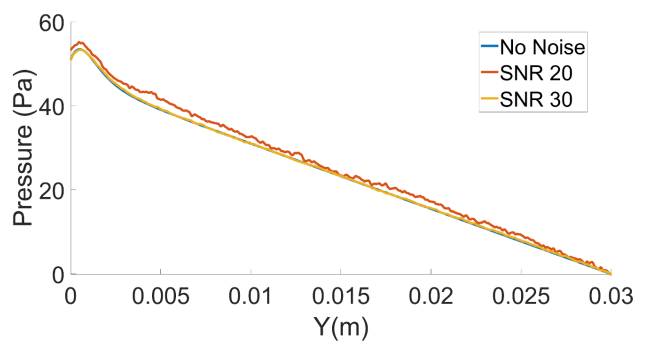

b

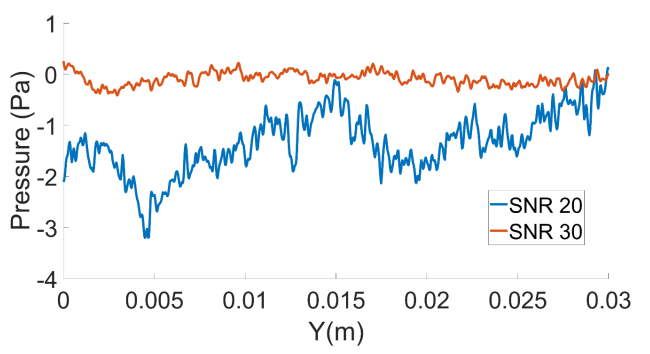

Figure 3.12: (a) Pressure drop along the axis of the channel in the case without noise and for SNR 20 and $30 \mathrm{~dB}$ (b) difference in the pressure drop along the axis of the channel between the case without noise and the case with noise (SNR 20 and $30 \mathrm{~dB})$.

\subsubsection{Temporal persistence of the enhancement}

In Figure $3.13 \mathrm{a}$ and $\mathrm{b}$ the velocity value and the velocity profiles in the non developed flow region $(\mathrm{y}=0.003 \mathrm{~m})$ at $t_{2}$ (last time step with the enforcement) and at 10 time steps following the removal of the external force are shown respectively. It is clear that after $t_{2}$, the effect on the enforcement starts to vanish and, as can be seen from the Figure 3.13 a, it takes 7 time steps for the system to converge towards the solution of the simulation without external force.

However the investigation of time resolved scenario goes beyond the scope of this work.

a

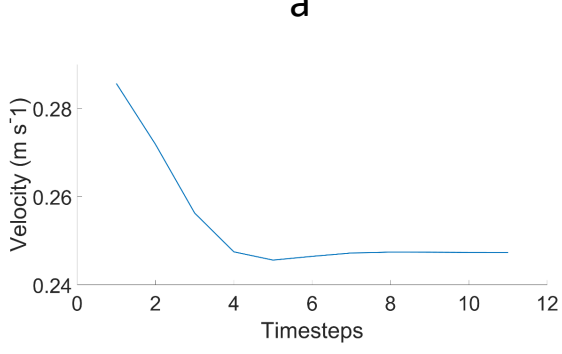

b

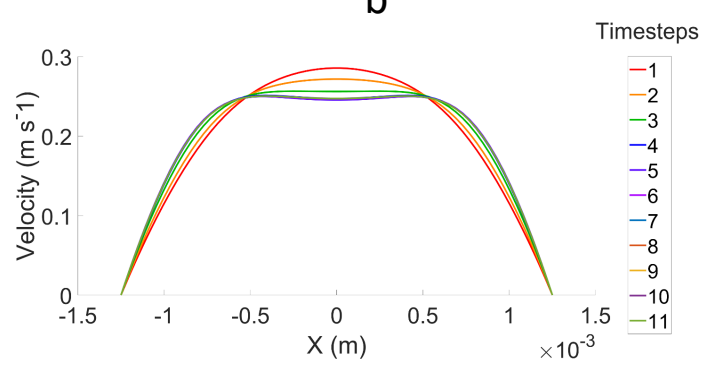

Figure 3.13: (a) Velocity value on the axis of the channel and (b) velocity profiles in the non developed flow region $(\mathrm{y}=0.003 \mathrm{~m})$ at $t_{2}$ (last time step with the enforcement) and at 10 time steps following the removal of the external force.

The results obtained in this section have the limitation of being in 2D; this does not allow to make more quantitative considerations on the velocity distribution, being the vector field a 3D entity. However this was a needed step in order to translate 
this approach from synthetic data to MRI data, and from an idealized geometry to a patient specific one. It has been proved that EMRI parameter $k$, influences the convergence time as well as the fluid dynamics of the system. In order to obtain sensible values of pressure, but at the same time inform the simulation using experimental data, $k$ should be kept equal to 1 . Moreover it was proved that using EMRI with data with the SNR of clinical 4D Flow CMR, not only corrects the flow field, but also gives an estimate of the pressure less than $1 \%$ different from the ideal case. In the next section EMRI will be tested on clinical 4D Flow CMR data.

\subsection{D EMRI}

In this section a 2D application of the EMRI method will be presented. The study focused on a part of a patient specific descending aorta, in order to test the methodology on real 4D Flow CMR data. Before introducing the EMRI 2D application, the methodology to post process the 4D Flow CMR data will be shown.

\subsubsection{D Flow CMR data processing}

The 4D Flow CMR data were processed using a in-house code written in Matlab environment (Matlab, MathWorks, Natick, MA), that allowed to retrieve the velocity components from the phase data, and to switch from the DICOM voxel to patient coordinate system, allowing the computation of the point of application of the external force in the CFD aortic domain.

In order to do so, the Digital Imaging and COmmunications in Medicine (DI$\mathrm{COM}$ ) format provides:

- Image position patient: that specifies the Cartesian coordinate of the upper left hand corner of the image, that is the centre of the first voxel of the MRI dataset.

- Image orientation patient: that specifies the direction cosines of the first row and the first column with respect to the patient.

- Pixel spacing attribute: refers to the row spacing and the column spacing in $\mathrm{mm}$. 
a

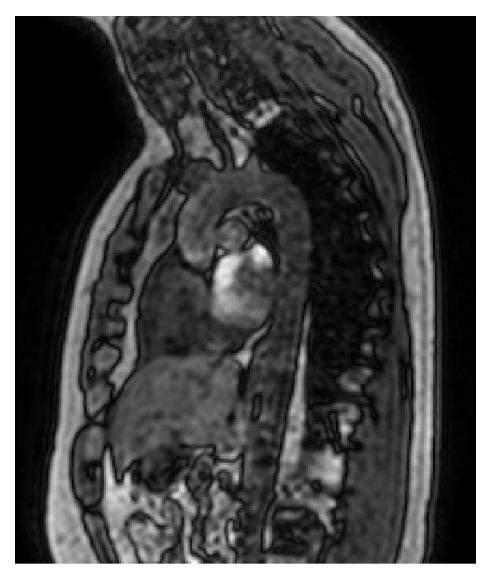

C

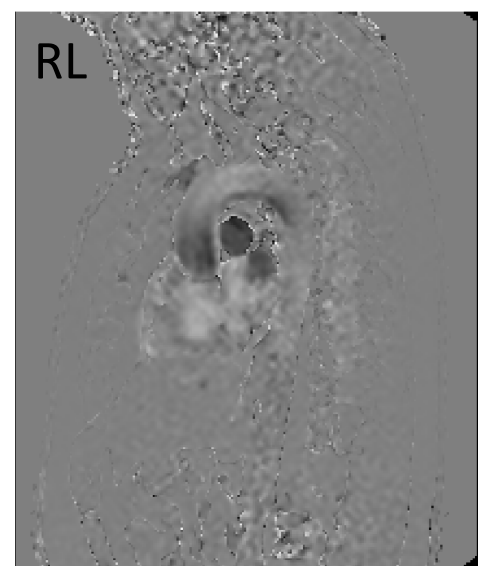

b

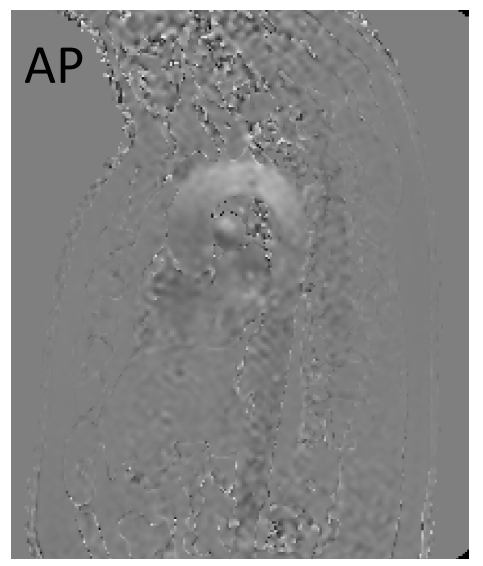

d

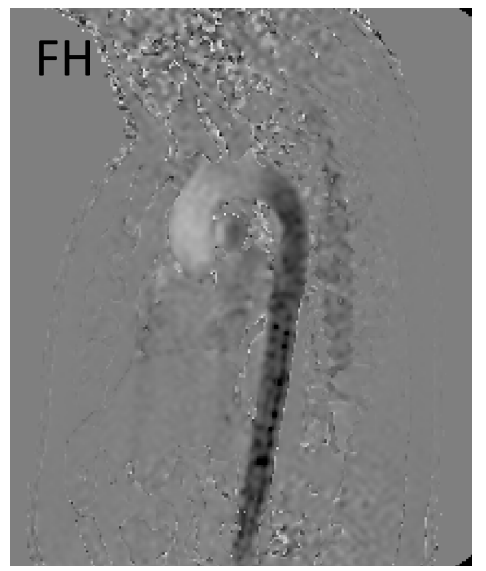

Figure 3.14: Slice of the 4D Flow CMR showing the magnitude (a), and the three phase encoding anterior- posterior (AP) (b), right-left (RL) (c) and feet-head (FH) (d) .

- Slice thickness: refers to the spacing between slices.

- $\mathbf{v}_{E N C}$ : the velocity encoding used in the sequence.

This information allows to perform the mapping of the image coordinate to the patient coordinate, writing the overall transformation $\mathbf{M}=\mathbf{T}_{1} \mathbf{R} \mathbf{S} \mathbf{T}_{0}$, where:

- $\mathbf{T}_{1}$ is the translation that locates the first pixel of the image at the image position of the patient,

- $\mathbf{R}$ is the rotation of the image coordinate that uses the direction cosines, 
- $\mathbf{S}$ is the scaling of the coordinate based on pixel spacing and slice thickness, and

- $\mathbf{T}_{0}$ is the translation of the first image voxel centre to the origin.

In order to extract the blood flow velocity, the grey scale image of the phase and the $\mathrm{v}_{E N C}$ value are used. In particular the following relationship (equation 3.9) holds:

$$
v=\frac{I_{p}-G S}{G S} \cdot v_{E N C}
$$

where $I_{p}$ is the intensity of the pixel and $G S$ is the grey scale of the phase image. In the Figure 3.14 the magnitude and the phases in the three encoding directions, anterior-posterior (AP), right-left (RL), feet-head (FH) are shown, for the case study.

\subsubsection{Materials and methods}

A healthy male volunteer was scanned with a cardiac gated 4D Flow CMR sequence (QFlow SENSE) on a 1.5 T MRI scanner (Achieva ${ }^{\circledR}$, Philips Medical Systems, Cleveland, $\mathrm{OH}$ ) at Hammersmith Hospital, London, UK. Prior to the scan, a written informed consent was obtained from the subject.

The parameters of sequence used were repetition time (TR) $4.4369 \mathrm{~ms}$, an echo time (TE $2.6335 \mathrm{~ms}$, flip angle $15^{\circ}$, matrix $256 \times 256$, pixel dimension $1.36 \times 1.36$ $\times 3 \mathrm{~mm}^{3}$, number of slices 17 , number of phases 20 .

Velocity encoding was made in three orthogonal directions: head-foot, rightleft and anterior-posterior with $v_{E N C}$ of $130 \mathrm{~cm} \cdot \mathrm{s}^{-1}$. The image sets were acquired at 20 equally-spaced intervals over the cardiac cycle, collecting 120 volumetric image sets (20 time points x 3 encoding directions x pairs of magnitude and phase images).

A 2D slice of the aorta was segmented from magnitude images, averaging the three encoding directions, using Simpleware ScanIP (version X.Y, Synopsys, CA, USA) and meshed using ICEM CFD (ANSYS Inc, Cannonsburg, PA, USA) using 
tetrahedral elements and prism elements for the boundary layer (10 layers) near the wall.

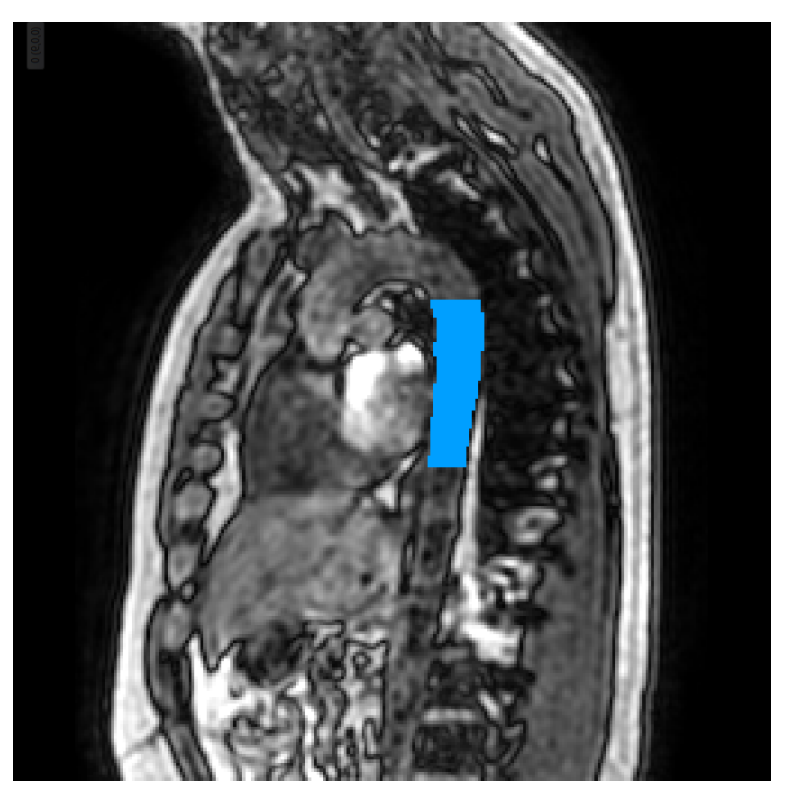

Figure 3.15: Slice of the MRI with the mask (in blue) of the region on which the study was performed.

In this study the flow was assumed to be laminar, incompressible and Newtonian, with density and viscosity equal to $1060 \mathrm{~kg} \mathrm{~m}^{-3}$ and $0.004 \mathrm{Pas}$ respectively. Afterwards an EMRI algorithm was applied using a uniform $k$. In particular in order to make use of the optimum value for $k$ obtained in the numerical verification $(k=1)$, the gain $K$ had to be scaled as this quantity is a function of the characteristic parameters of the system $U, L$ and of the viscosity $\rho$. If $U_{0}, L_{0}$ and $\rho_{0}$ are the characteristic velocity, length and density of the system studied in the numerical experiment respectively, and $U, L$ and $\rho$ are the same quantities for the segment of aorta considered, the scaling factor is given by Equation 3.10:

$$
s=\frac{\left(\rho_{0} / \rho\right)\left(U_{0} / U\right)}{L_{0} / L}
$$

The segment of the descending aorta considered had a $L=0.0178 \mathrm{~m}$, and an instant during late systole $(t=0.2348 s$ from the start of the cardiac cycle, refer to Figure 3.16) was selected for this test. The average velocity at the inlet was $U=$ $0.4997 \mathrm{~m} \cdot \mathrm{s}^{-1}$. Therefore the scaling factor $s=2.85$. 


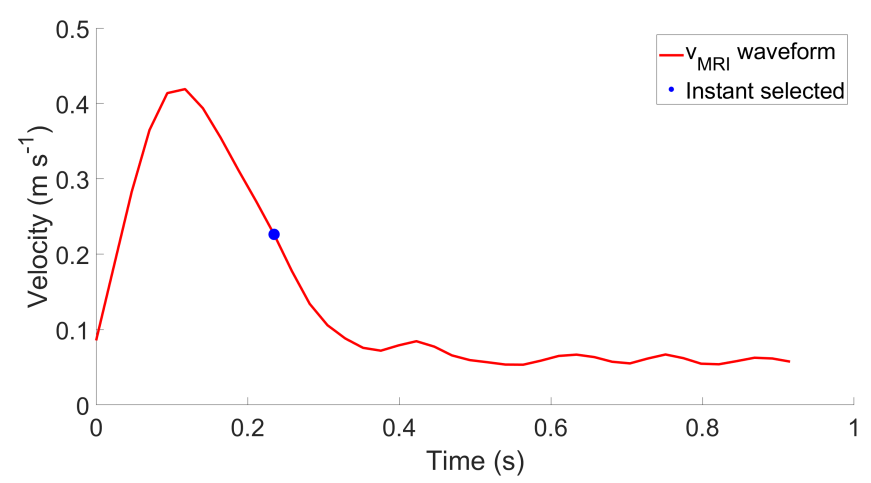

Figure 3.16: Velocity waveform of the aorta at the inlet and instant selected for the $2 \mathrm{D}$ analysis.

Cartesian velocity profiles were used as inlet boundary conditions in both the EMRI and the CFD. The outlet boundary condition was placed at the exit of the descending aorta. The vessel's wall was assumed rigid and no-slip boundary condition was applied.

The correct coordinate transformation was assessed by (1) plotting the mapped MRI data and the nodes of the CFD model mesh and (2) comparing the velocity retrieved directly form the MRI data to the one imported in the CFD model. Afterwards the velocity maps estimated with EMRI were compared with those derived with 4D Flow CMR and CFD.

\subsubsection{Results and discussion}

\subsubsection{MRI coordinate mapping}

Figure 3.17 a, shows the plot of the mapped MRI data and the nodes of the CFD model (reduced by $50 \%$ to allow for visual comparison). It is clear that the coordinate mapping leads to a perfect overlap between the CFD model geometry and the MRI data.

In order to assess the correctness of the velocity values, the velocity maps retrieved directly from the 4D Flow CMR data and the velocity map resulting from the linear interpolation of the 4D Flow CMR velocity data on the CFD mesh (Figure $3.17 \mathrm{~b}$ ). Also in this case, there is perfect agreement in the spatial distribution of the velocity values as well as in their values, confirming the goodness of the mapping. 
a)

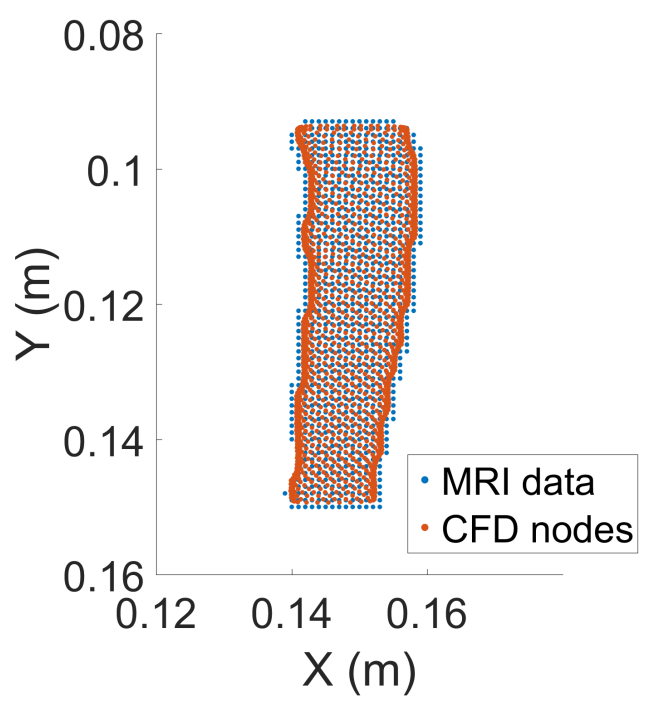

b)

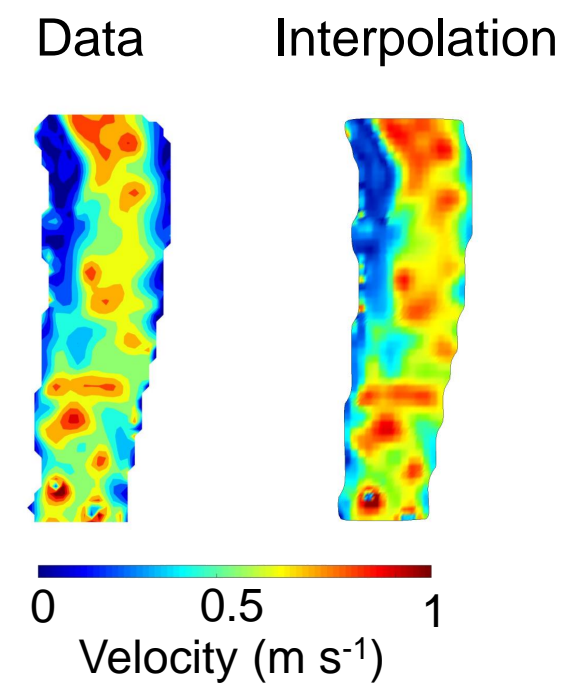

Figure 3.17: (a) Plot of the mapped MRI data (indicated as MRI data) and the mesh nodes of the CFD model. (b) Velocity maps retrieved directly form the MRI data (indicated as data) and velocity maps interpolation on the domain of the CFD model.

\subsubsection{Velocity field comparison}

The in plane velocity map measured with 4D Flow CMR, and computed with CFD and EMRI are presented in Figure 3.18.

The difference between the flow field computed is evident. In particular the following aspects could be considered:

- Spatial resolution: the 4D Flow CMR shows its resolution limitation, especially if compared with CFD and EMRI.

- Velocity field: comparing CFD and 4D Flow CMR velocity field it could be noticed how the velocity patterns present evident differences. On the other side the EMRI enables to embed the 4D Flow CMR obtained velocity field features. This is shown clearly in Figure 3.21 where the velocity difference map is presented.

The EMRI is able then to provide high resolution results, capturing the experimental velocity field features. There is another main advantage of EMRI: the capability of 


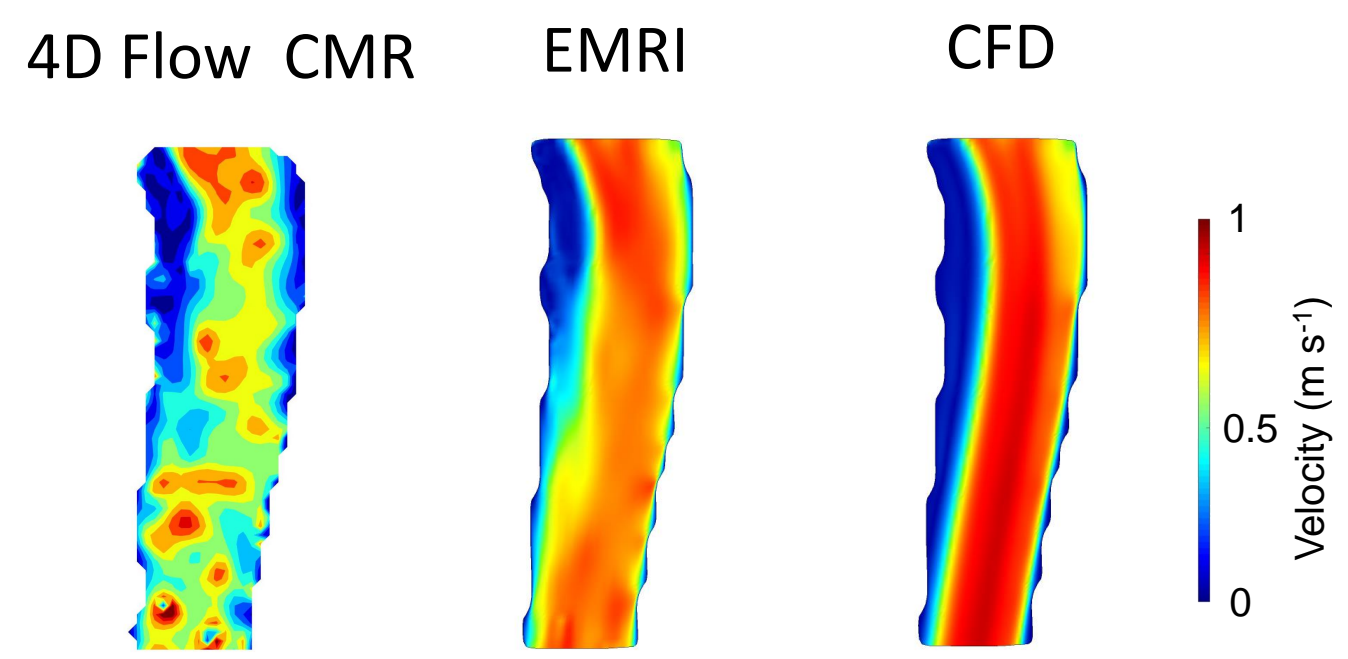

Figure 3.18: Velocity maps of the region of the descending aorta computed with the MRI, CFD and EMRI.

4D Flow CMR

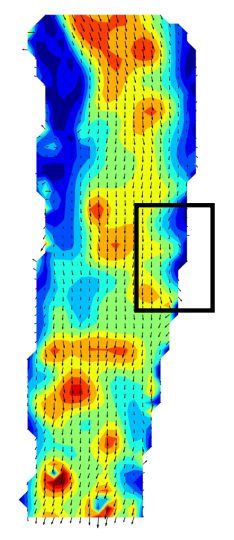

EMRI
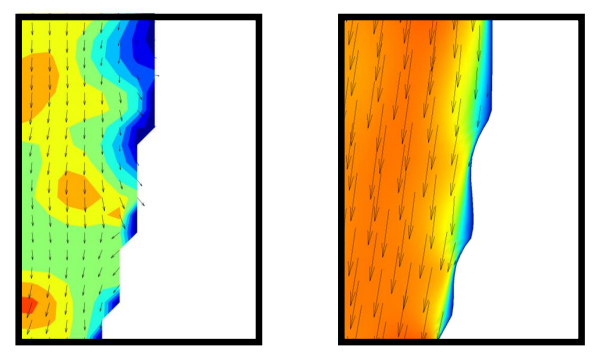

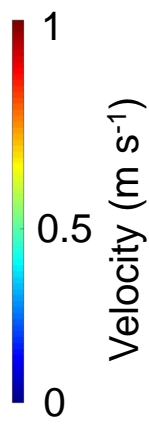

Figure 3.19: Velocity maps with vectors of the region of the descending aorta computed with the MRI, CFD and EMRI.

providing solutions that satisfy the laws of fluid dynamics.

In order to enlighten this aspect, the velocity vectors of 4D flow CMR and EMRI are showed (Figure 3.19). The EMRI analysis enables to correct velocity vectors that are generated most likely by noise, like in the portion enlarged in Figure 3.19. It is clear that the non-physical velocity vectors obtained using raw 4D Flow CMR data 
a)

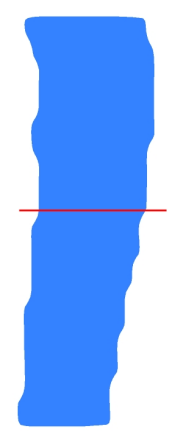

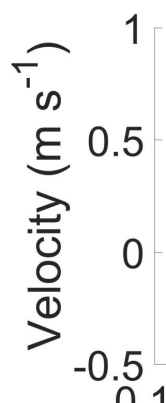

0.14

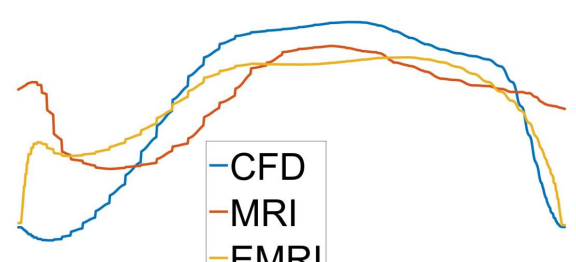

EMRI b)
$0.145 \quad 0.15$
0.155
0.16

Figure 3.20: Section of the aortic portion (a) where the velocity profiles measured with 4D Flow CMR, and computed CFD and EMRI where compared.
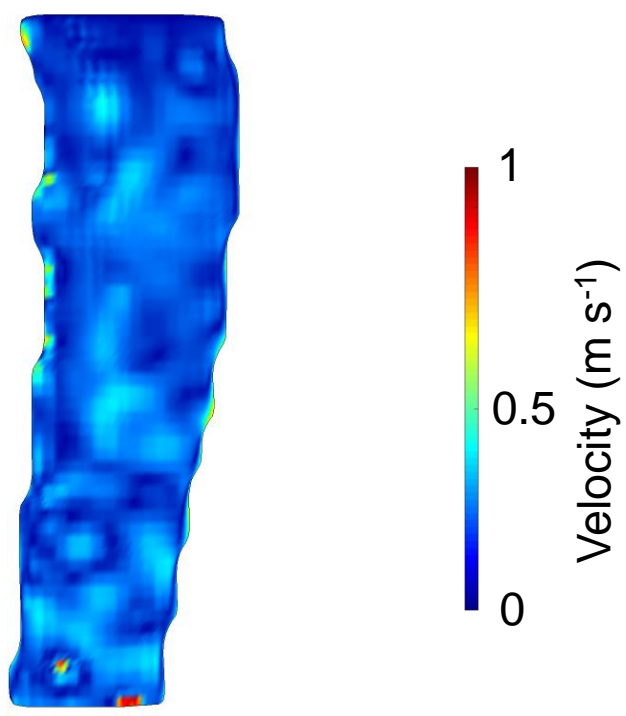

Figure 3.21: Velocity difference map between the 4D Flow CMR measured velocity and the one computed with EMRI.

$[41,69]$, non-zero on the wall of the domain are corrected by the EMRI approach, leading to a divergence free velocity field.

A quantitative confirmation is given by: 
- the velocity difference map Figure 3.21 that shows an heterogeneous pattern with peaks in the region close to the wall and in some specific point of the domain (these last are most likely caused by phase encoding errors)

- the comparison of the velocity profile obtained with 4D Flow CMR, CFD and EMRI along a line crossing the descending aorta. 4D Flow CMR velocity values are non-zero on the aortic wall, in contrast with the two computational models that correctly fulfil the non-slip boundary condition.

These features will impact dramatically the cardiovascular markers that could be estimated from the 4D Flow CMR, such as WSS. In fact EMRI high resolution and physically sound results will lead to a more accurate estimate of velocity fields and velocity dependent quantities.

A necessary step to extend this approach to a 3D patient specific case was the validation of EMRI, which would be shown in the next chapter. 


\section{Chapter 4}

\section{Validation of EMRI: assessment of}

\section{the flow field in a $\mathrm{U}$ bend using $4 \mathrm{D}$}

\section{Flow CMR, CFD, 2D PIV and EMRI}

In the previous chapters the concept of EMRI was presented and tested firstly in 2D in a channel, to investigate the behaviour of the model parameters, and afterwards in a 2D portion of the human descending aorta to study the feasibility of the suggested method on real 4D Flow MR data. However an important step needed to bring forward this methodology is its validation.

As mentioned before, PIV is a technique which is widely used to quantify flow fields with high spatial and temporal resolutions. In the recent years, with the development of sophisticated computational fluid dynamics models, the aforementioned technique has become widely used to provide a reference velocity field $[79,78]$.

Indeed PIV is an ideal imaging tool for providing a reference velocity field because it is a non-intrusive quantitative technique capable of capturing instantaneous complex flow structures with high spatial resolution. The aim of this chapter is to validate EMRI using 2D PIV, by fulfilling the following objectives:

1. to build a test rig compatible with PIV and MRI;

2. to compare the flow patterns measured with 4D Flow CMRI, PIV and computed with CFD, to highlight their advantages and limitations; 
3. to validate the EMRI against PIV and to highlight its potential to bring further the concept of measure informed computational modelling.

The methodology for the fabrication of phantoms presented in this chapter has been published in the ASME Journal of Engineering and Science in Medical Diagnostics and Therapy [1]. The validation study has been included in another paper submitted to Journal of IEEE Transactions on Medical Imaging (comparison), and have been presented at the 8th World Congress of Biomechanics and at the Virtual Physiological Human (VPH) 2020 (allowing to gain the Medtronic Travel Grant).

\subsection{Material and Methods}

\subsubsection{Test rig}

In order to have a test rig allowing to perform 4D Flow CMR and PIV measurements, the system needed to be:

- MRI compatible: no ferromagnetic parts had to be used in the MRI scanner room.

- PIV compatible: the phantom had to be made in a transparent material and with the same refractive index as the liquid used.

To ensure the MRI compatibility, no ferromagnetic parts had to be used in the test rig. Therefore the pump used had to be placed outside of the MRI scanner room, and therefore long pipes were used and 20 litres of working fluid were necessary.

Given the intrinsic difficulties of realizing PIV-compatible phantoms a simple shape was chosen; in particular a $U$ bend was used as an idealized and simplified model of the aortic arch. The transparency requirement was addressed by manufacturing the phantom with a clear, solvent free, low viscosity silicone elastomer (MED 6015, NuSil Technology, CA, USA) with a refractive index $n=1.4$.

A novel manufacturing process was adopted [1], involving three stages (Figure 4.2): (1) Creation of the geometry; (2) 3D printing in Poly Vinyl Alcohol (PVA); (3) Casting and dissolving of PVA in water. 


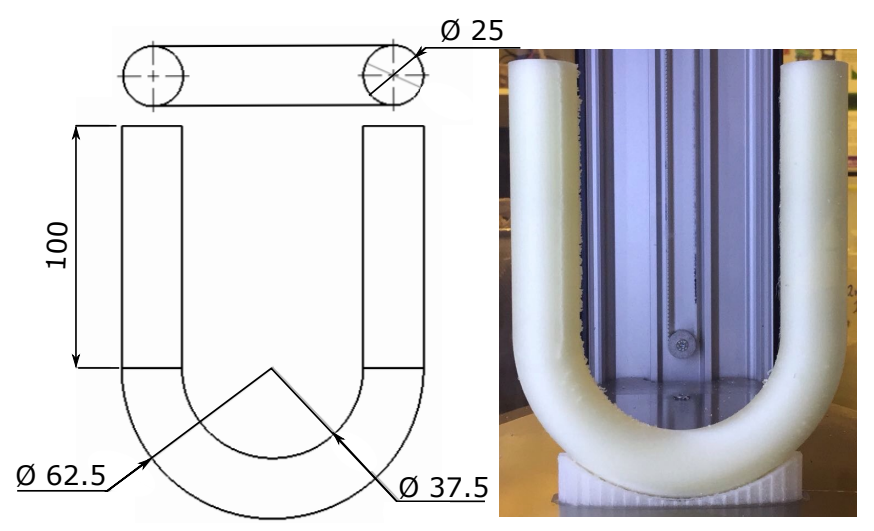

Figure 4.1: Geometry and PVA mould of the U bend pipe.

The mould for the $\mathrm{U}$ bend (lumen diameter $D$ of $0.025 \mathrm{~m}$, curvature radius of $0.05 \mathrm{~m}$ ) was designed with a CAD software (SolidWorks, Dassault Systems, Canada) .

The $\mathrm{U}$ bend lumen volume was 3D printed in PVA (Figure 4.1) using a stereolithography (SLA) printer, in particular a Delta WASP 2040 Turbo 2 (Wasproject, Massa Lombarda, Italy). PVA is commonly employed as a support material to create complex geometries in Polylactic acid (PLA), which is then dissolved in water to obtain the object of interest. However, in this approach, PVA was used as the primary material to create a mould for the casting process (i.e. negative mould).

Attention was paid to the choice of the infill, one of the most important factors for $3 \mathrm{D}$ printing (i.e. the structure printed inside the object). A higher infill will result in a stronger model, which is especially important in the case of a complex structure, but it will also lead to increased duration of the PVA dissolving process. To find the best compromise, different simplified test sections were 3D printed varying the infill parameter $(10 \%-60 \%)$ and the shell thickness ( $1 \mathrm{~mm}-3 \mathrm{~mm})$, leading to the final choice of $30 \%$ infill and shell thickness of $2 \mathrm{~mm}$.

The 3D printed model, constituting the negative mould, was further post processed before the casting procedure. In particular a transparent acrylic spray paint (PlastiKote, UK) was used to make the contact surface with the casting material, impermeable and hydrophobic, so as to reduce chemical interactions that could have affected the silicone optical properties.

The final stage in the manufacturing process was the casting. The model was 


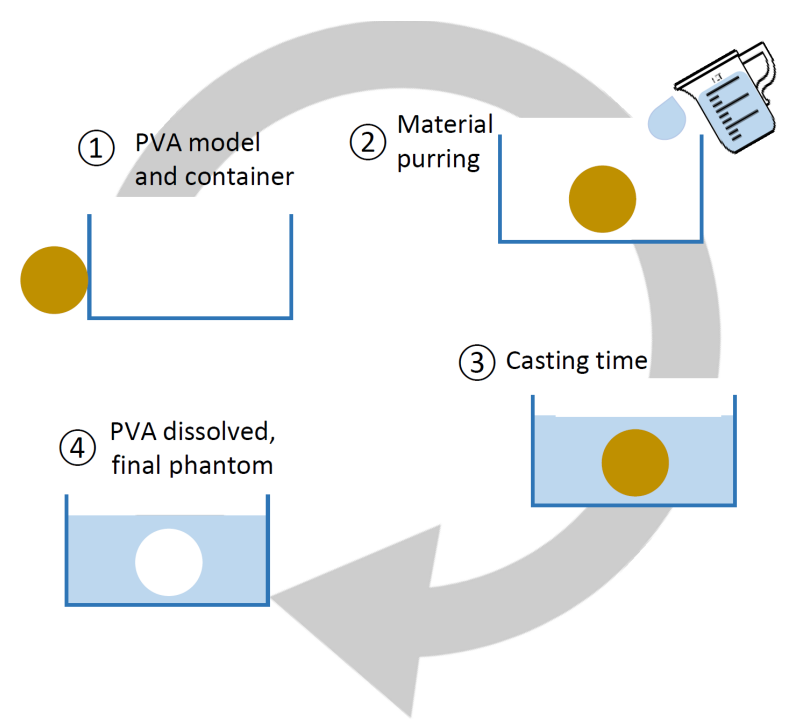

Figure 4.2: Schematics of the casting : the PVA model is enclosed in a box and afterwards the silicone is poured, which results in a hollow structure once the material is dissolved.

enclosed in a rigid box (manufactured in clear cast acrylic, Perspex) and the casting material was pored to create a box with a hollow structure reproducing the lumen. The curing of the silicone required 48 hours, and subsequently the object was placed in a water bath to let the inner PVA mould dissolve.

Finally the phantom was placed back in the rigid box to ensure a rigid behaviour during the experiments and in order to connect the phantom to the flow circuit.

To minimise optical distortion the refractive index of the working fluid has to match that of the silicone $U$ bend pipe. A mixture of water and glycerol (60\% by weight of the solution, leading to a refractive index of $n=1.4$ [98] ), was adopted (Figure 4.4), resulting in a liquid with a density and viscosity of $1150 \mathrm{~kg} \cdot \mathrm{m}^{-3}$ and $0.008 \mathrm{~Pa} \cdot \mathrm{s}$ respectively. In Figure 4.3 the optical distortions before and after the refractive index matching are shown in a silicone phantom used for testing purposes, and in the final $U$ bend phantom used in the experiments. The density and viscosity of the fluid used are $8 \%$ more and twice as much as the one of blood, respectively. This choice was driven by the fact that the amount of fluid needed was $20 l$ and the use of glycerine and water kept the costs reasonable and posed no health and safety 


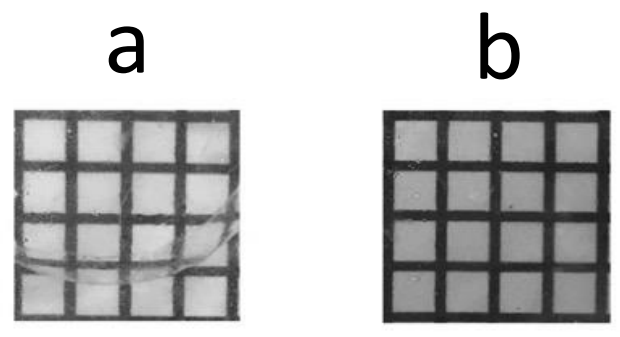

C

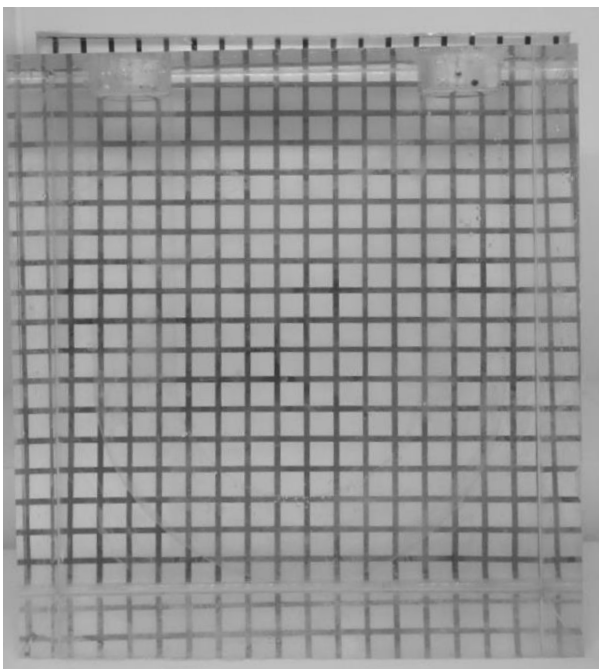

Figure 4.3: A silicone phantom used for testing purposes before (a) and after (b) the refractive index matching. The square grid at the back shows the optical distortions due to the difference in the refractive index of the silicone and the surrounding fluid. (c) U bend phantom filled with the working fluid with the same refractive index of the silicone, with the square grid at the back, showing the absence of optical distortions. 
issues.

Hollow glass particles with a nominal diameter was $10^{-5} m$ (Dantec Dynamics HGS-10) were dispersed in the fluid, in order to scatter any light incident upon them. Their density was of $1100 \mathrm{~kg} \cdot \mathrm{m}^{-3}$, leading therefore to a $50 \mathrm{~kg} \cdot \mathrm{m}^{-3}$ difference with respect to the density of the working fluid. This would result in a gravitationally induced velocity of the order of $10^{-6} \mathrm{~m} \cdot \mathrm{s}^{-1}$, well outside of the expected range of measured velocity.

A pulsatile flow was generated by means of a Blood Pump (Harvard Apparatus, UK) (Figure 4.9), which was operated at $60 \mathrm{bpm}$, with a systole diastole ratio of $40 / 60$, which are in the same range as physiological ones. However the stroke volume chosen was $40 \mathrm{ml}$, which is smaller than the physiological one $(70 \mathrm{ml})$. The resulting Womersley number $(\alpha)$ was 12.23 and the peak Reynolds number $(R e)$ was 977.8; in aortic flow these are in the ranges $16-20$ and 3500-4500, respectively.

The parameters had to be adjusted in order to fulfil the following requirements:

- have a rigid phantom without exerting to much force on the perspex box (which was held together by plastic screws) and avoid spillage of working fluid in the MRI scanner.

- have flow in laminar regime, to fit the validation purpose of the experiment.

The pump, the $\mathrm{U}$ bend and the working fluid reservoir were connected in series according to the diagram shown in Figure 4.5.

\subsubsection{PIV Experimental set-up}

A TSI PIV 2D Measurement system (TSI Inc., Minnesota, USA) was used to perform 2D PIV, which gives access to a high resolution velocity maps in the imaged plane. Images were captured by a camera placed orthogonally to the plane of measurement which is illuminated by a laser sheet ( of thickness $0.4 \mathrm{~mm}$ ) (Figure 4.7). The plane chosen is in the middle section of the $U$ bend, and it is indicated in Figure 4.6 .

A spatial resolution of $0.3717 \mathrm{~mm}$ ( 67.5 points across the phantom diameter) was achieved by subdividing the $\mathrm{U}$ bend into 9 different regions of interest (Figure 

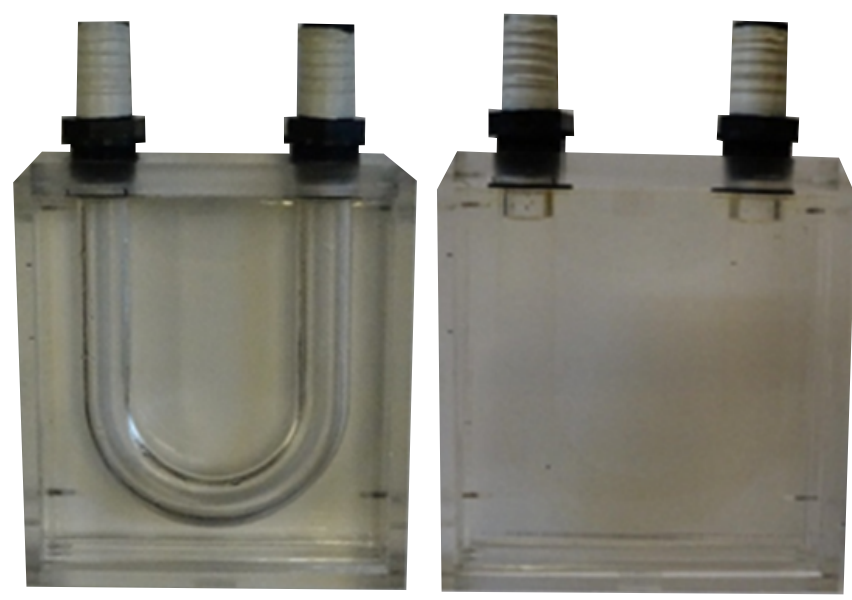

Figure 4.4: $\mathrm{U}$ bend silicone phantom without (on the left) and with (on the right) refractive index matched fluid.

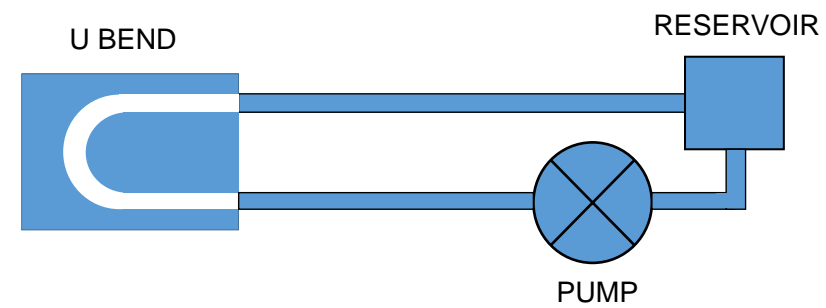

Figure 4.5: Diagram of the experimental rig.

4.8) which were imaged separately and merged together during the post processing.

Using a synchroniser, the PIV system was connected to the pump trigger in order to repeatedly capture images from specific instants within the pump cycle, allowing for phase-averaging over multiple image pairs (Phase resolved PIV). The phase resolved velocity maps were obtained at four instants of the pump cycle, three during systole (where relevant flow changes are expected) and one in diastole. These instants are shown in Figure 4.13), and correspond to the points of $57 \mathrm{~ms}$ (1), $145 \mathrm{~ms}$ (2), $233 \mathrm{~ms}$ (3) and $527 \mathrm{~ms}$ (4) from the start of the systole. The phase resolved velocity maps were obtained by taking an ensemble average of 100 cycles for each of the 4 time points.

Each acquisition consists of two images, corresponding to each of the two laser pulses fired, one at time $t$ and one at time $t+\Delta t$, calculated according to the the 


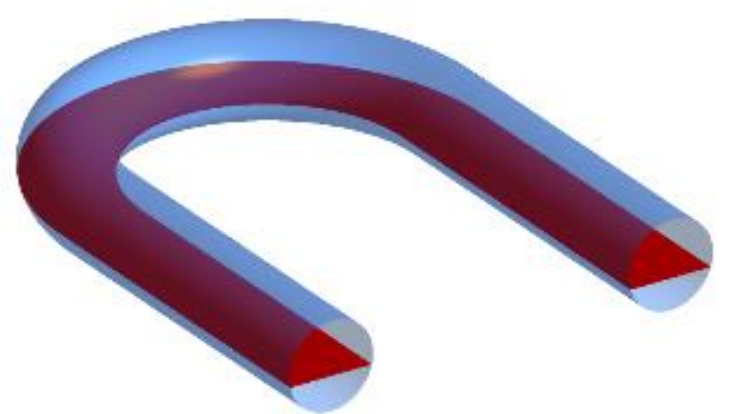

Figure 4.6: Phantom with the plane selected for the comparison.

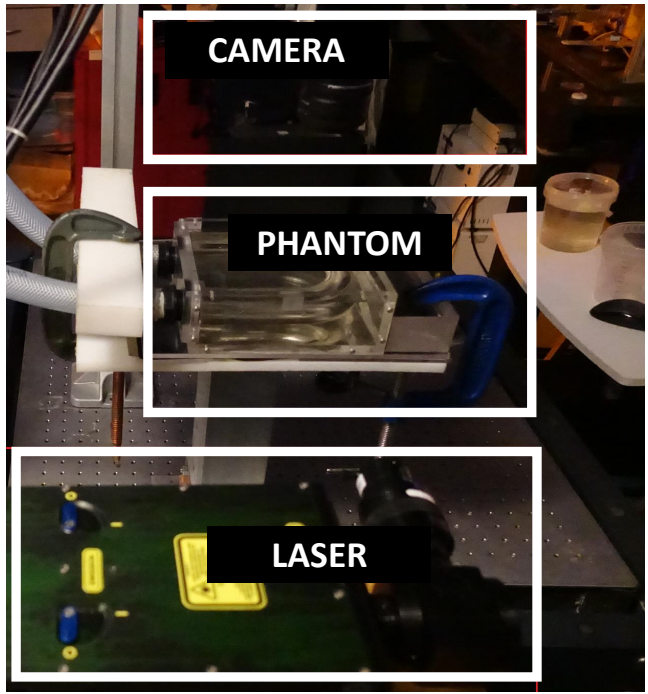

Figure 4.7: Diagram of the piv experimental setup. 


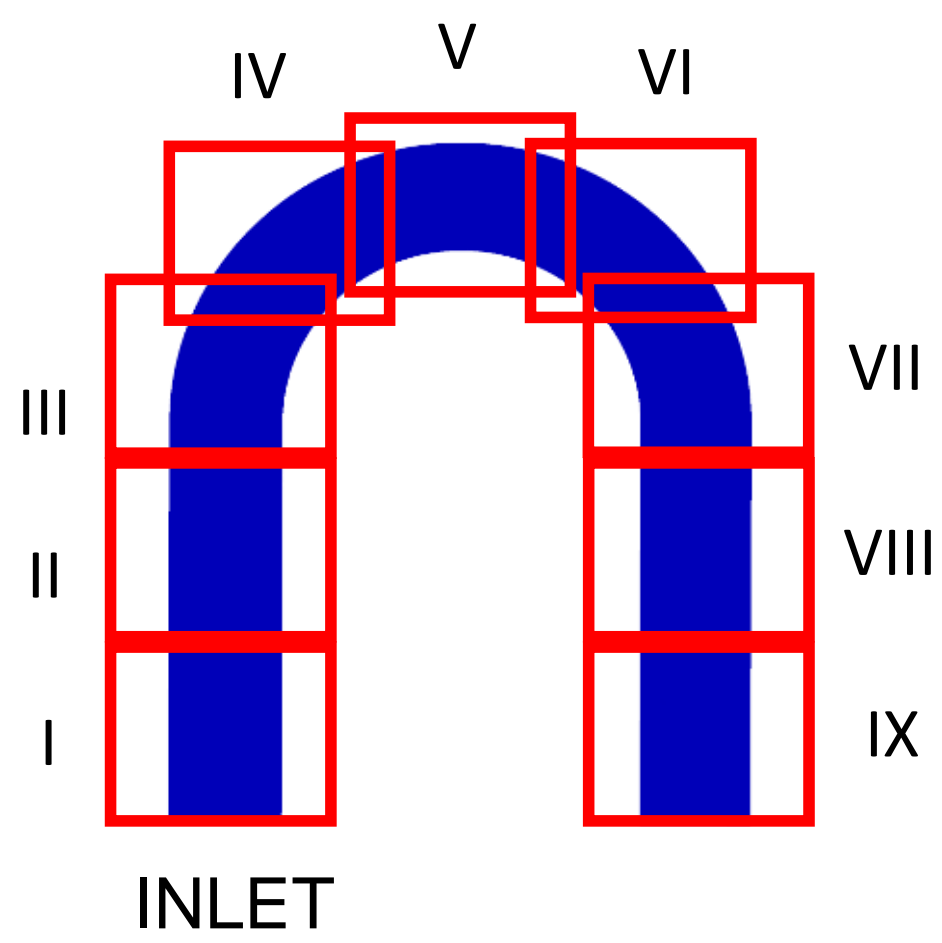

Figure 4.8: $U$ bend with the 9 regions of interest imaged separately during the PIV experiment.

one-quarter rule [77]. This rule says that 'the maximum displacement of a particle should not exceed 1/4 of the size of the interrogation spot' (Equation 4.1):

$$
\Delta t=\frac{n_{\text {spot }} \cdot R}{4 \cdot v_{\max }}
$$

where $n_{\text {spot }}$ is number of pixels of the final interrogation spot (32 $\times 32$ pixels), $R$ is the $\mu m$ to pixel ratio defined during the calibration process of the system (20.5 $\mu \mathrm{m} /$ pixel $), v_{\max }$ is the maximum velocity expected at the time point selected. Therefore according to the point of the cycle considered, the $\Delta t$ (the laser delay) was varied in the range 300 - $500 \mu s$ ((1): $500 \mu s$, (2): $300 \mu s,(3): 300 \mu s$ and (4): $500 \mu s)$.

The images capture with the PIV system were subsequently processed using Insight $4 \mathrm{G}^{T M}$ (TSI Inc., US). In particular the processing step comprised:

- pre-processing: during this step $R$ is determined via a calibration step, that 
relates the physical length of the object imaged and the pixels. Afterwards, in order to save computational time, a mask is applied to the images to select the area of interest which would be subsequently processed to determine the velocity field. Lastly, a Gaussian low-pass filter was applied to the image to blur the particles and make easier the recognition during the processing.

- processing: the pre-processed images are divided in small spots for the computing of the velocity vectors. In particular the image pairs are processed in two subsequent steps, using first a spot of $64 \times 64$ pixels used to compute an average displacement which is then used as initialization for the second step which adopts a $32 \times 32$ pixels spot to compute the displacements for which the cross correlation between the two images is maximum.

- post-processing: at this stage the computed vectors are 'validated' by mean of comparison with the mean velocity in a $5 \times 5$ neighbourhood, in order to filter out the displacement vector exceeding a tolerance threshold (2 pixels). The last step of the post processing allows to recover the displacement values in pixels that fail the previous step. In particular the mean vector of neighbourhood vectors is used in areas with a minimum of 3 'good' neighbour vectors in a $5 \times 5$ neighbourhood.

- velocity field: the 100 instantaneous flow fields, each obtained at the same instant during the cycle, are then averaged.

The PIV velocity data are intrinsically affected by an error, which may be introduced at different phases of the measurement pipeline. They fall in three main categories: the errors caused by system components (e.g. installation and alignment, timing and synchronization error, particle tracing capability, illumination), those due to the flow itself (velocity gradients, fluctuations) and those caused by the evaluation technique.

In order to minimize the experimental errors, attention was paid to the selection of the working fluid in order to minimize the refraction of the laser light and the 
particle used, as previously explained. The background noise was minimized by isolating the test rig from external light sources.

The out-of-plane motion, which could cause unwanted movements of the seeding particles out of the interrogation area between the image pair was minimized by choosing a suitable $\Delta t$.

The velocity uncertainty due to the random error on the estimate of the displacement field with a finite interrogation window could be calculated as (Equation 4.2):

$$
\varepsilon_{v}=\frac{0.1 \cdot R}{\Delta t}
$$

which assumes that the maximum absolute uncertainty on the displacement is 0.1 pixel [77].

However the use of a 'universal constant' is very simplistic as the uncertainty could vary greatly from experiment to experiment, with space and time within the same experiment, and is heavily dependent on the flow to be imaged, the imaging system, the experimental conditions and the image processing [99].

A simple but effective way to assess the accuracy of the computed velocity profile is the analysis of mass conservation [100]. Assuming axial symmetry of the velocity profile (which in this case is a reasonable assumption as the captured images are in the middle plane of a cylindrical tube), and constant viscosity, the conservation of mass requires that the flow rate is consistent across the sections of the chosen ROI.

Taking into consideration a region $A$ in the inlet segment of the $\mathrm{U}$ bend, where $A$ is a $S \times P$ matrix, with $S$ the number of sections, and $P$ the number of pixels across the section $(s=1, \ldots, S, p=1, \ldots, P)$ the average flow rate $F L_{S}$ at each instant is given by (Equation 4.3):

$$
F L_{s}=\sum_{p}^{P} \pi x_{p} v_{s, p} d_{p i x}
$$

where $x_{i}$ is the position along the diameter, $v_{s, i}(t)$ is the axial velocity at the 

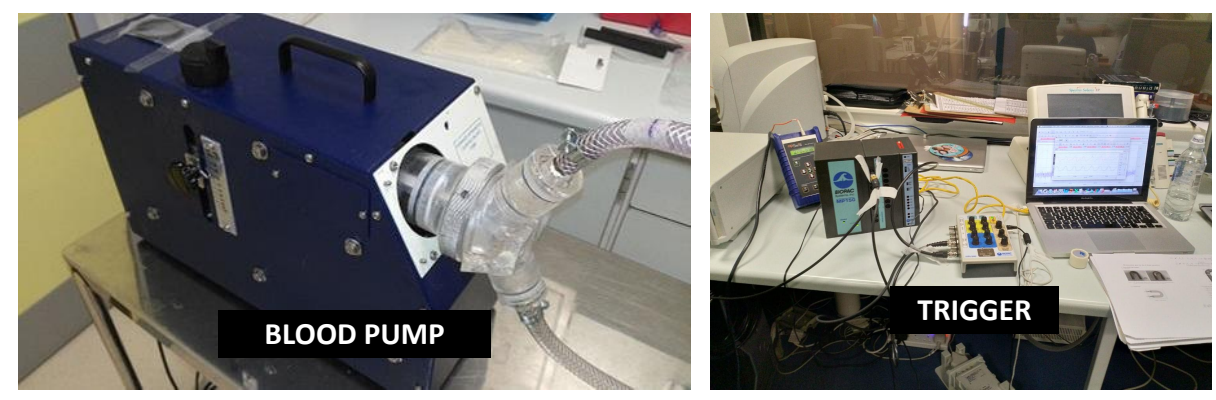

Figure 4.9: Photo of the blood pump and of the MRI control room during the experiment.

s-th section and i-th pixel, and $d_{p i x}$ is the pixel dimension.

The minimum flow rate $F L_{\min }$ and the maximum flow rate $F L_{\text {max }}$ were calculated to compute the percentage error $\varepsilon_{P I V}$ using Equation 4.4:

$$
\varepsilon_{P I V}=\frac{F L_{M a x}-F L_{\min }}{F L_{\max }}
$$

The velocity maps obtained for each of the $U$ bend parts were merged together using Matlab (The Mathworks, Natick, MA, USA).

\subsubsection{D Flow CMR}

The U bend was scanned with a cardiac gated 4D Flow CMR (Siemens) on a $1.5 \mathrm{~T}$ MRI scanner (Avanto MRI scanner, Siemens, Erlangen, Germany) at Great Ormond Street Hospital for Children (NHS Foundation Trust, London, UK).

The MRI acquisition was triggered by the blood pump, and the parameters of the sequence used were TR $26.64 \mathrm{~ms}$, TE $3.67 \mathrm{~ms}$, flip angle $15^{\circ}$, matrix $160 \times 160$, pixel dimension $1.875 \times 1.875 \times 1.9 \mathrm{~mm}^{3}$, number of slices 30 , number of phases 32. Velocity encoding was made in three orthogonal directions: head-foot, right-left and anterior-posterior with $v_{E N C}=50 \mathrm{~cm} \cdot \mathrm{s}^{-1}$.

The image sets were acquired at 32 equally-spaced intervals over the pump cycle, corresponding to 192 volumetric image sets (32 time points x 3 encoding 
directions x pairs of magnitude and phase images), subsequently processed with an in-house Matlab code.

To assess the accuracy of the velocity field measured with the 4D Flow CMR, the standard deviation of the velocity was calculated using equation 4.5 [101]:

$$
\sigma_{v}=\frac{\sqrt{2} \cdot V_{E N C}}{\pi S N R}
$$

where SNR is the signal-to-noise ratio of the magnitude images, calculated as the ratio of the average pixel intensity in two equally sized regions of interest, one in the $\mathrm{U}$ bend lumen (experiencing phase change) and the other in a stationary region. The standard deviation was assessed at the inlet, and in the three parts where the velocity profiles comparison were performed (Figure 4.15).

Another important parameter to assess the quality of the velocity mapping is the $V N R$ that is defined as $4.6[101]$ :

$$
V N R=\frac{v}{\sigma_{v}}
$$

where $v$ is the measured velocity. As the $v_{E N C}$ value is set to exceed the peak velocity, this value is usually very high compared to velocity in diastole, which leads to a decrease of the VNR.

\subsubsection{EMRI and CFD}

The CAD geometry of the phantom was registered to the MRI magnitude images and meshed using ICEM CFD (ANSYS Inc, Cannonsburg, PA, USA). After running a grid independence test, the geometry (Figure 4.6) was meshed using tetrahedral elements and prism elements for the boundary layer (10 layers) near the wall. The mesh has 727498 nodes and 1697646 elements.

An EMRI algorithm was performed using MRI retrieved flow rate as boundary conditions. Constant pressure outflow boundary condition (set to $0 \mathrm{~Pa}$ ) was prescribed at the outlet of the $U$ bend. The flow was assumed to be laminar and incompressible and the fluid Newtonian, with density and viscosity equal to 1150 $\mathrm{kg} \cdot \mathrm{m}^{-3}$ and $0.008 \mathrm{~Pa} \cdot \mathrm{s}$ respectively. Walls were assumed to be rigid and a no-slip 
boundary condition was applied. The force coefficient $k$ was set to 1 in the domain. In order to do so, as in the case of the $2 \mathrm{D}$ aortic segment, a scaling factor $s$ had to be applied (Equation 3.10), taking into consideration the characteristic length $(L=0.025 \mathrm{~m})$ and the characteristic velocity (refer to Figure 4.13 ) of the $U$ bend, and the density of the working fluid. The values of $s$ are reported in Table 4.1.

Table 4.1: Gain scaling $s$ at the different instants of the pump cycle considered.

\begin{tabular}{|c|c|}
\hline \hline Instant & $s$ \\
\hline 1 & 12.289 \\
2 & 7.374 \\
3 & 6.583 \\
4 & 23.043 \\
\hline \hline
\end{tabular}

For comparison a standard time resolved CFD analysis was also carried out over three cardiac cycles (with a time step of $0.001 s$ ). A time dependent and fully developed parabolic velocity profile was imposed at the inlet. The average velocity of the parabolic profile at each time step was set consistent to the measured flow waveform (shown in red in Figure 4.13, together with the one measured with PIV in blue). At the exit of the tube an outlet boundary condition with $0 P a$ pressure was applied.

The simulations were conducted using a commercial computational fluid dynamic solver ANSYS CFX (version 18.0, ANSYS Inc. Cannonsburg PA, USA) using a standard desktop workstations (Intel Core i7 6700K 4GHz, 16GB RAM, 8 cores).

The velocity maps of the longitudinal plane of the $U$ bend (Figure 4.6) estimated with EMRI were compared with those derived from MRI, CFD and validated against 2D PIV, in the selected instants of the cycle (Figure 4.13).

Furthermore the 4D Flow CMR, CFD, EMRI and PIV velocity profiles were compared in three sections across the PIV plane in each of the 4 instants considered (Figure 4.15). In order to compare 4D Flow CMR measured, and CFD and EMRI computed velocities, the relative difference $\Delta$ with PIV measured velocity was computed at each pixel of the considered sections at the four time instants, 
using Equation 4.7:

$$
\Delta_{p}=\frac{\left|v_{p}^{P I V}-v_{p}^{C}\right|}{\left|v_{\max }^{P I V}\right|}
$$

The relative percentage difference of each section was also computed (Equation 4.8):

$$
\Delta=\sum_{p}^{P} \frac{1}{P} \frac{\left|v_{p}^{P I V}-v_{p}^{C}\right|}{\left|v_{\max }^{P I V}\right|}
$$

where $p=1, \ldots, P$ with $P$ the number of pixels across the section, and $v_{p}^{P I V}$ and $v_{p}^{C}$ the velocity at the $p-t h$ pixel measured with PIV and computed (with CFD or EMRI) or measured with $4 \mathrm{D}$ Flow CMR, respectively. $v_{\max }^{P I V}$ is the maximum velocity measured with PIV at each section and time instant. Finally, although the purpose of this chapter is EMRI validation, the cross sectional velocity maps at the entrance $\left(0^{\circ}\right)$ and at the exit $\left(180^{\circ}\right)$ of the $\mathrm{U}$ bend are shown (Figure 4.22).

\subsection{Results and discussion}

The validation of the EMRI approach was carried out in a U bend geometry. Based on the theory, when a fluid is moving in a straight pipes towards a bend, the fluid particles will change their direction of motion. In fact the curvature generates a pressure gradient that causes a decrease of velocity close to the inner side of the bend and an increase of it in the outer part of it. Moreover the centrifugal force originates a secondary flow, and as a consequence a flow from the inner curvature to the outer curvature occurs around the symmetry plane of the pipe, and the flow comes back inward along the wall [102]. This effect in combination with the pressure difference between the outer and inner wall causes the well-known Dean vortices in the cross section.

The flow regime is characterize by a Womersley number $(\alpha)$ of 12.23 , a peak Reynolds number $R e_{\text {peak }}$ of 977.8 and an average Reynolds number $R e_{\text {mean }}$ of 456.3 . In unsteady condition, the critical Reynolds number for the onset of turbulence decreases with the increase of the Womersley number, and the ratio $R e / \alpha=200$ could be used as a threshold [103]. In this study this ratio at the peak velocity is 89 , 
largely below that threshold, therefore the flow could be considered in the laminar regime.

Another parameter used to assess the onset of turbulence is the critical Reynolds number $R e_{c r i t}$ as empirically found by Peacock et al. [104], computed as (Equation 4.9):

$$
\operatorname{Re}_{\text {crit }}=169 \alpha^{0.83} S t^{-0.27}
$$

where $S t$ is Strouhal number, calculated as (Equation 4.10):

$$
S t=\frac{D f}{2\left(v_{\text {peak }}-v_{\text {mean }}\right)}
$$

where $D$ is the characteristic length of the system (in this case the diameter), $f$ is the pump cycle frequency, and $v_{\text {peak }}$ and $v_{\text {mean }}$ are respectively the peak and the mean velocity magnitude of the cycle. In the studied system the $R e_{\text {crit }}=2576.6$, therefore the system regime is expected to be laminar.

The unsteady flow in curved pipes depends not only by Reynolds and Wommersley number but also by the ratio between the pipe curvature radius and the radius of the pipe $R_{c}$, that in the considered geometry is 4 . Even though the assessment of the flow patterns in curved pipes with steady and pulsatile flow could be found in literature $[102,105,106]$, studies with the exact same flow conditions and geometry are not present. Moreover this type of investigation goes beyond the objective of this work that focused on the velocity field in the middle plane of the bend with the aim of comparing four different techniques.

Before comparing the outcomes of the different methodologies used, some examples of the PIV and 4D Flow CMR data are shown.

In Figure 4.10 the raw and processed PIV data of the region II of the U bend (refer to Figure 4.8) are presented: Figure 4.10 a, shows the raw picture captured by the camera in the mid plane, and the particles are clearly visible. In Figure $4.10 \mathrm{~b}$ the extracted vectors are presented in the full image and in Figure $4.10 \mathrm{c}$ a magnification of a smaller portion of the region of interest is shown with the purpose of showing 
Table 4.2: $\Delta t$ (the laser delay), $\varepsilon_{P I V}$ (the maximum velocity uncertainty) and $\varepsilon_{p i v}$ of the PIV measurements at the different instants of the pump cycle .

\begin{tabular}{|c|c|c|c|}
\hline \hline Instant & $\Delta t(\mu s)$ & $\varepsilon_{v}\left(m s^{-1}\right)$ & $\varepsilon_{P I V}(\%)$ \\
\hline 1 & 500 & $0.41 \cdot 10^{-3}$ & 11.41 \\
2 & 300 & $6.83 \cdot 10^{-3}$ & 4.11 \\
3 & 300 & $6.83 \cdot 10^{-3}$ & 4.28 \\
4 & 500 & $0.41 \cdot 10^{-3}$ & 6.73 \\
\hline \hline
\end{tabular}

the vectors retrieved.

In Table 4.2 the theoretical maximum velocity uncertainty $\varepsilon_{v}$ (Equation 4.2) and the percentage error $\varepsilon_{p i v}$ (Equation 4.4, computed using $S=30$ ) is shown for each instant of the pump cycle selected for the PIV experiment.

It is clear that the theoretical error is underestimating the uncertainty on the measured velocities, whereas the percentage error $\varepsilon_{p i v}$, given the test rig geometry gives a robust way to asses the uncertainty. The higher value obtained for the percentage error is at the first time instant, due to the flow disturbances in this portion of the U bend.

In Figure 4.11 the magnitude (M), and the three phase encoding maps (anteriorposterior (AP), right-left (RL) and feet-head (FH)) of one slice of the 4D Flow CMR data at the time instant 2 are shown. In Figure 4.12 the velocity map and the velocity vectors overlaid on the velocity contour plot are shown for the slice in Figure 4.11.

In Table 4.3 the SNR, the VNR and the $\sigma_{v}$ of the 4D Flow CMR in the instant of the pump cycle considered for the comparison, are presented.

Table 4.3: SNR, VNR and $\sigma_{v}$ of the 4D Flow CMR data at the different instants of the pump cycle considered.

\begin{tabular}{|c|c|c|c|}
\hline \hline Instant & SNR & VNR & $\sigma_{v}\left(m s^{-1}\right)$ \\
\hline 1 & 12.04 & 4.07 & 0.0415 \\
2 & 16.46 & 5.64 & 0.0443 \\
3 & 15.71 & 8.3 & 0.0318 \\
4 & 5.91 & 0.376 & 0.0845 \\
\hline \hline
\end{tabular}

A comparison of velocity maps and velocity profiles in the symmetry plane and in selected cross sections of tube was carried out. 


\subsubsection{Velocity maps}

The average velocity at the inlet of the $\mathrm{U}$ bend during the pump cycle, measured with 4D Flow CMR and PIV (with the standard deviation) in the section considered (Figure 4.6), is plotted in Figure 4.13.

The average cross sectional velocity shows a good agreement between the two sets of experimental data. This is particularly true during systole while in diastole the PIV data deviates from the 4D Flow CMR. In this phase of the pump cycle the velocity magnitude is much lower than the $\mathrm{v}_{E N C}$ and as a consequence the velocity encoded with MRI is less accurate, as it is shown by the trend of the $v \pm \sigma_{v}$ (dashed lines in Figure 4.13).

Figure 4.14 shows the velocity maps in the selected plane (Figure 4.6) at the 4 selected instants, measured with 4D Flow CMR and PIV and computed with CFD and EMRI.

Overall there is a good qualitative agreement of the velocity distribution between all the different modalities.

The first time instant is at the beginning of the systole. Considering PIV as the reference flow, the velocity magnitude in the outlet section and in the inner part of the arch is lower in the 4D Flow CMR measurement results. On the other hand CFD shows much higher velocities in the whole domain. On the contrary EMRI velocity map is much closer to the PIV in terms of velocity magnitude and flow patterns.

A similar agreement can be identified when comparing the different sets of data at time instant 2. Although the 4D Flow CMR retrieved velocity map shows good similarity to the PIV one, the outlet branch and the arch are characterized by lower velocities. CFD and EMRI show very good agreement with PIV in terms of velocity magnitude, however the flow patterns are better captured by EMRI that corrects for the velocity drop that characterizes MRI in the low velocities areas, while preserving the flow structures.

In the third instant considered, the velocity distribution is consistent across all the approaches, experimental, computational and hybrid. However PIV shows less flow separation in the arch with respect to the other techniques. 
The last point considered is in the diastole of the pump cycle. The flow patterns are in good agreement. Nonetheless the CFD analysis presents higher velocity in the inlet branch, while the MRI shows lower velocity in the arch.

\subsubsection{Velocity profiles}

In order to have a quantitative comparison between the different approaches, the velocity profiles in three sections of the selected plane of the $U$ bend (Figure 4.15) at the 4 instants of time considered, were compared (Figure 4.16 - 4.18).

Overall 4D Flow CMR shows lower velocities in comparison to PIV (adopted as the reference field), as also remarked during the analysis of the velocity maps. This is clearly evident in section B and C at instant 1 and 2 .

On the other hand the CFD analysis leads overall to slightly higher velocities compared to PIV: section A instants 3 and 4, section C instant 2. Moreover CFD leads to a different velocity distribution in almost all the sections considered.

In most of the cases EMRI velocity profiles show a good agreement with the ones measured with PIV. In particular the velocity profiles show consistency with the reference ones in particular at instants 1,2 and 4 in section A, at instants 1,2 and 4 in section $\mathrm{B}$, and 2 and 3 in section $\mathrm{C}$.

Even in the cases in which the 4D Flow CMR-derived velocities were much lower than the PIV ones, like in section B instant 1 and 2, and in section $\mathrm{C}$ instant 1, EMRI corrected for these low velocities, producing flow patterns comparable to the PIV ones. Using Equation 4.7, the relative difference between the velocity measured with PIV and the one computed with CFD/EMRI or measured with 4D Flow CMRI were computed in all the sections considered at the four instants of time (Figure 4.19 - 4.21).

In most of the cases the highest differences between the velocity computed and measured are in the region near the wall, as PIV suffers from errors in the velocity estimation in these areas [107].

While CFD and EMRI show relative differences within the same range in all the sections, 4D Flow CMR relative difference is higher in sections B and C.

In the Tables $4.4-4.5-4.6$ the relative difference of the velocity magnitude 
is computed for all the time instants at the different sections comparing CFD with PIV, EMRI with PIV and MRI with PIV respectively.

In section A the agreement between EMRI and PIV is better with respect to CFD in all the instants except the third one. In this case the EMRI overestimates the velocity as could be seen in Figure 4.16. In this section the 4D Flow CMR has the higher agreement with the reference flow in the systole, while in diastole the relative difference is higher.

Also in section B the EMRI has a lower relative difference with respect to CFD and 4D Flow CMR in all the time instants but the forth one, where the $\Delta$ is an order of magnitude higher with respect to the other instant and is higher than the one of MRI.

In section $\mathrm{C}$ the relative difference of CFD and PIV is lower than the one between EMRI and PIV in all the instants except the second one. Therefore the CFD performs better compared to the EMRI even though the relative differences are overall comparable.

Table 4.4: $\Delta_{P I V-C F D}$ for each section at the different instants of the pump cycle considered.

\begin{tabular}{|c|c|c|c|}
\hline \hline \multicolumn{4}{|c|}{$\Delta_{P I V-C F D}$} \\
\hline Instants & Section A & Section B & Section C \\
\hline 1 & 0.1208 & 0.1590 & 0.1300 \\
2 & 0.0873 & 0.0741 & 0.1508 \\
3 & 0.1557 & 0.1720 & 0.1136 \\
4 & 0.3457 & 0.5589 & 0.2569 \\
\hline \hline
\end{tabular}

Table 4.5: $\Delta_{P I V-E M R I}$ for each section at the different instants of the pump cycle considered.

\begin{tabular}{|c|c|c|c|}
\hline \hline \multicolumn{4}{|c|}{$\Delta_{P I V-E M R I}$} \\
\hline Instants & Section A & Section B & Section C \\
\hline 1 & 0.0971 & 0.0704 & 0.1949 \\
2 & 0.0652 & 0.0715 & 0.0875 \\
3 & 0.1969 & 0.0634 & 0.1583 \\
4 & 0.0981 & 0.6364 & 0.2713 \\
\hline
\end{tabular}

Table 4.7 summarises the results, showing the $\Delta$ averaged over the sections at the time instants selected. 
Table 4.6: $\Delta_{M R I-P I V}$ for each section at the different instants of the pump cycle considered.

\begin{tabular}{|c|c|c|c|}
\hline \hline \multicolumn{4}{|c|}{$\Delta_{P I V-M R I}$} \\
\hline Instants & Section A & Section B & Section C \\
\hline 1 & 0.1367 & 0.3349 & 0.5410 \\
2 & 0.0699 & 0.3082 & 0.3457 \\
3 & 0.0728 & 0.1575 & 0.1565 \\
4 & 0.2822 & 0.4212 & 0.5201 \\
\hline
\end{tabular}

Overall EMRI computed velocities show higher agreement with the PIV ones with respect to CFD and 4D Flow CMR, and in particular in the systole. In diastole the relative difference is doubled. The CFD relative difference shows the same trend of EMRI, while the 4D Flow CMR relative difference is much larger in the accelerating phase of the systole and in diastole.

Table 4.7: $\Delta$ for each time instant of the pump cycle considered, averaged over the sections considered.

\begin{tabular}{|c|c|c|c|}
\hline \hline Instant & $\Delta_{P I V-C F D}$ & $\Delta_{P I V-E M R I}$ & $\Delta_{P I V-M R I}$ \\
\hline 1 & 0.1366 & 0.1208 & 0.3375 \\
2 & 0.1041 & 0.0748 & 0.2413 \\
3 & 0.1471 & 0.1395 & 0.1289 \\
4 & 0.3872 & 0.3353 & 0.4078 \\
\hline \hline
\end{tabular}

\subsubsection{Cross sectional flow}

The cross sectional velocity maps in the sections showed in Figure 4.22 are shown for the four instants of time in Figures 4.23 and 4.24.

The velocity maps show good agreement at the $0^{\circ}$ section in the 4 instants selected. At instant 1 the velocity distribution of the EMRI is less symmetric with respect to the one of CFD, showing a similar distribution with respect to the $4 \mathrm{D}$ Flow CMR. At instant 4 while EMRI has a flow distribution similar to the MRI one, the CFD shows higher velocities in the centre of the lumen, as in this case the profile has negative velocities in the boundary layer as could be seen in Figure 4.16 at instant 4 .

The velocity maps in the cross section at $180^{\circ}$, presented in Figure 4.24, show overall a consistency in the velocity distribution. However the 4D Flow CMR data 
in the instants 1,2 and 4 shows lower velocity magnitude compared to the other modalities. It should be also considered the overall velocity decrease between $180^{\circ}$ with respect to what observed in the section at $0^{\circ}$.

\subsection{Discussion and limitation of the study}

This validation study highlights the main flaws of 4D Flow CMR. In particular it is evident that the sensitivity of the MRI sequence to low velocities is highly compromised by the chosen $v_{E N C}$. This results in some deviation of the 4D Flow CMR data from the PIV profiles, with an effect which is more pronounced during diastole.

In the MRI sequence used, the temporal resolution was selected such that cardiac cycle was covered by 32 time frames, and the acquisition consisted of two data sets for each directional velocity encoding. This allowed to average the velocities of each time point of the cycle, increasing the signal to noise ratio (SNR) [8]. However dividing the cardiac cycle in temporal intervals causes an averaging of the signal over these time intervals. Therefore the velocity estimated by 4D Flow CMR results averaged and therefore lower than expected. This limit leads also to underestimation of peak velocities, as observed in systole [41].

Another reason behind the lower velocities measured is the fact that the depth of the voxel is determined by the slice thickness. Usually this is the largest dimension of the voxel, due to the fact that the thicker the slice the higher the SNR. Being the slice thickness $1.9 \mathrm{~mm}$ the velocities measured in the plane bisecting the $\mathrm{U}$ bend are averaged over this thickness, giving rise to velocities underestimations [37].

Another main limit of 4D Flow CMR is the partial volume effect, predominant in the region close to the wall, since in these regions velocity gradients are very high and as a consequence a single voxel contains a broad distribution of velocity [11].

The decrease of velocity magnitude between inlet and outlet segment, confirmed by the cross sectional velocity maps, is prominent in all the instants apart from instant 3; it could find its justification in two facts:

- the $U$ bend used, even though made in rigid silicone and enclosed in a rigid 
box, had a compliant behaviour.

- in order to connect the system to the blood pump, placed in the control room, long pipes and pipe fittings were needed, which affected the pressures at the inlet and outlet of the bend.

Also the computational models present limitations. Most of the time, CFD overestimates velocities. Moreover in some cases the experimental features of velocity profiles are not well captured. This is the case of the non parabolic flow structure present in the inlet segment. This feature is consistently present in the velocity maps measured with PIV and 4D Flow CMR, and is likely to be originated by the pipe coupling with the $U$ bend. This feature is correctly reproduced by the EMRI, and it is not captured by the CFD model. This is the direct consequence of the simplifications introduced by choosing the idealized parabolic shaped velocity profile at the inlet. These approximations are common in computational studies for clinical applications $[108,109]$ and might lead to substantially different flow paths and flow derived parameters.

Morbiducci et al. [13] highlight how the choice of the inlet boundary conditions plays a major role in computational studies, showing the impact on the CFD model outcome of a patient specific aorta when imposing the full 3D velocity field and the axial one as inlet boundary condition. This study highlights how enhancing computational models incorporating in vivo measurements of velocity profiles could be a necessary step in the pipeline for subject-specific analysis of haemodynamics.

Part of the limits of 4D Flow CMR and CFD are mitigated by EMRI. The poor sensitivity of 4D Flow CMR to low velocities is compensated by the need of satisfying the mass conservation, leading to a fluid dynamic sound velocity distribution also in diastole.

Moreover the non-slip condition and the presence of high resolution mesh in the boundary layer characterising EMRI allows to correct the velocities in proximity of the wall, which in the 4D Flow CMR case are more likely to be affected by a large error. This is a characteristic which is present in all the velocity profiles presented, 
and it is reported in literature; this feature is of fundamental importance especially when velocities in proximity of the wall are used for the retrieval of flow derived parameters (e.g. WSS) in clinical environment $[51,63]$.

Overall the agreement between EMRI and PIV is satisfactory. Some discrepancies might be observed in the velocity magnitude in section A instant 3 , section $\mathrm{B}$ and $\mathrm{C}$ instants 3 and 4 . The first reason behind this discrepancy might be the wrong estimate of flow rate with 4D Flow CMR, especially in the area of low velocity, where the signal-to-noise ratio is low as the $v_{E N C}$ is larger than the velocity of the fluid.

Secondly it should be taken into account that also the EMRI model is characterized by rigid walls, which might lead to the velocity discrepancy with respect to the PIV. In fact the compliance of the silicone elastomer used to fabricate the $\mathrm{U}$ bend might have led to changes in the diameter during data acquisition. Consequently the enlargement of the lumen due partly to the internal pressures and partly to the outlet pressure caused by the long pipes used could be at the origin of the discrepancies in the average velocities between PIV and the computational models [110].

Lastly, it should be considered that PIV is characterized by experimental uncertainties. In particular:

- errors in the phantom fabrication, connected also to the $3 \mathrm{D}$ printing of the mould used for the phantom.

- the PIV velocity maps of the $\mathrm{U}$ bend were obtained as a result of 9 different experiments. Even if these experiments were conducted all in one session, to limit the change in the experimental conditions, the camera and the laser had to be moved to image each of the $\mathrm{U}$ bend pipe portions. Moreover after the post processing of each section, the velocity maps had to be registered to the $\mathrm{U}$ bend geometry, to obtain the full $\mathrm{U}$ bend velocity map.

The last limitation of this study concerns the use of PIV as reference technology for velocity near the walls. In fact also for PIV, a similar consideration to the one done for 4D Flow CMR applies, presenting non-zero velocity on the wall, as 
visible in the velocity profiles of the different sections and instants presented. This is clearly showed also by the relative difference profiles, where the boundary layer of PIV measurements is often characterized by higher relative difference values with respect to the bulk flow. This error might be linked to the non-uniform particles distribution in proximity of the wall and the Gaussian low-pass filter applied during the processing, that makes easier the recognition of the particle in the subsequent processing steps but biases the locations of the correlation peak in the case of near-wall flow investigations [107]. Nonetheless, even in these areas, if compared with 4D Flow CMR, PIV flow field shows values of velocity very close to zero, justifying the choice of this method as a validation tool. 

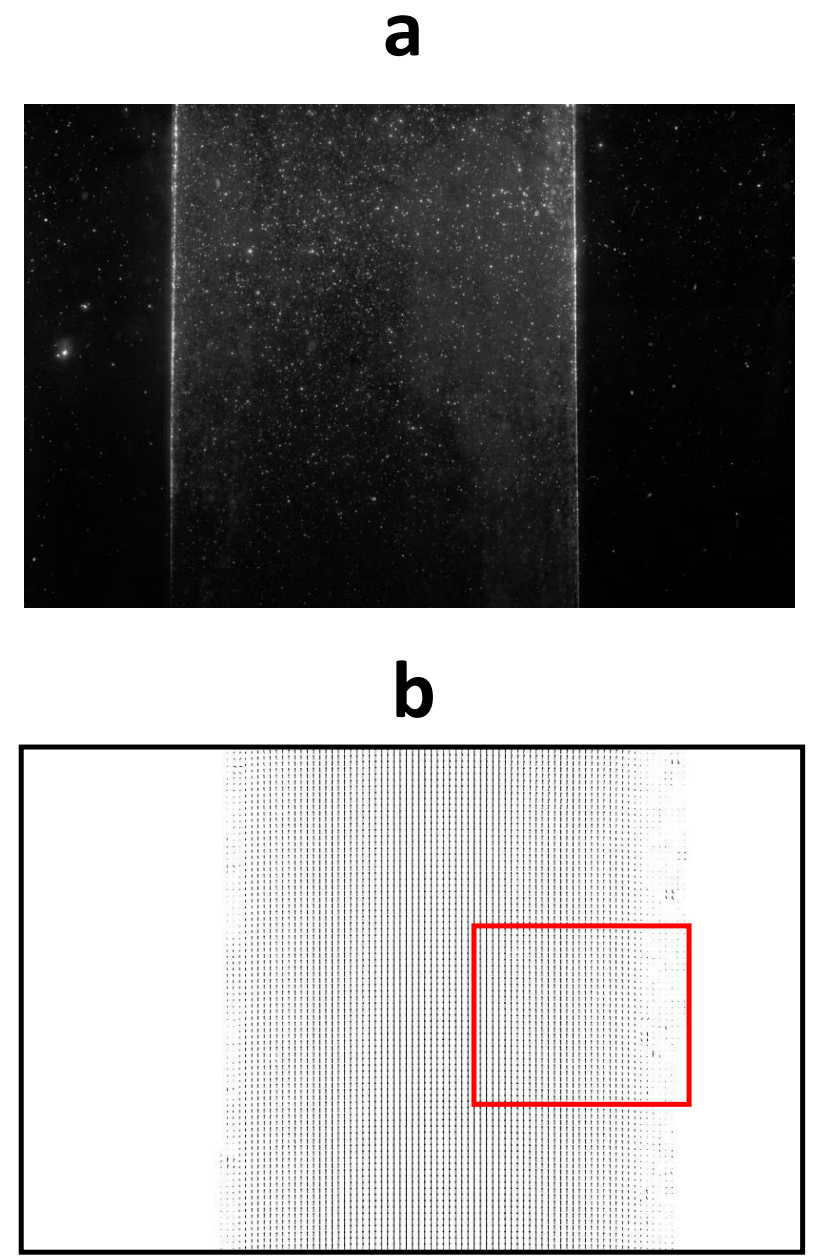

C

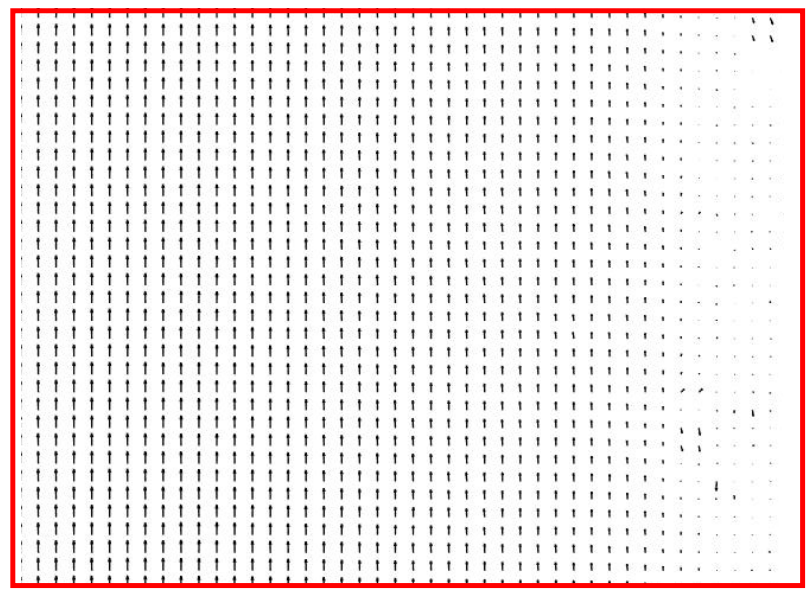

Figure 4.10: PIV raw data (a), processed vectors (b) of the region II of the U bend, with a magnification of one portion of it (c). 

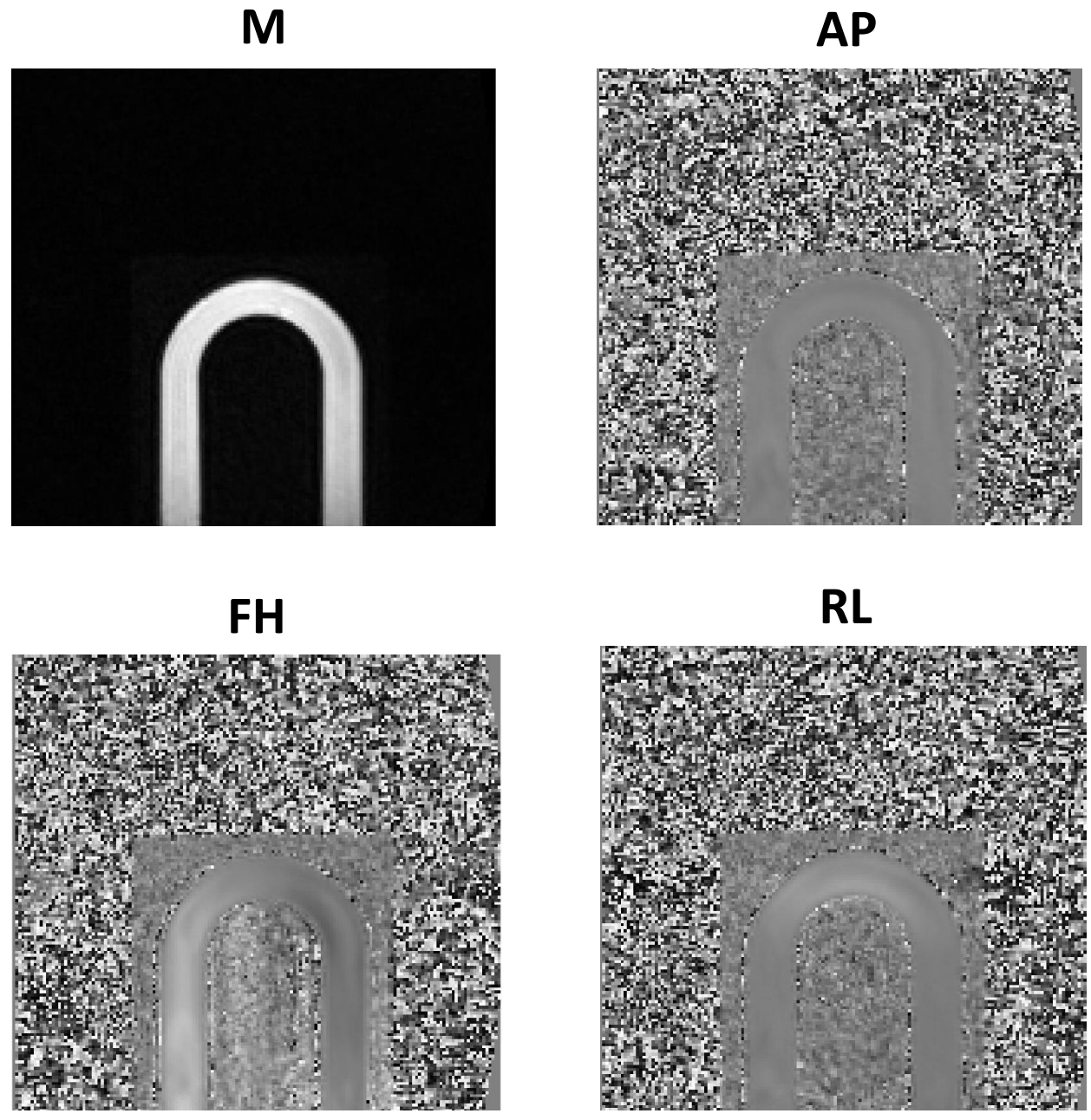

Figure 4.11: Slice of the 4D Flow CMR showing the magnitude (M), and the three phase encoding anterior-posterior (AP), right-left (RL) and feet-head (FH) at the time instant 2 . 

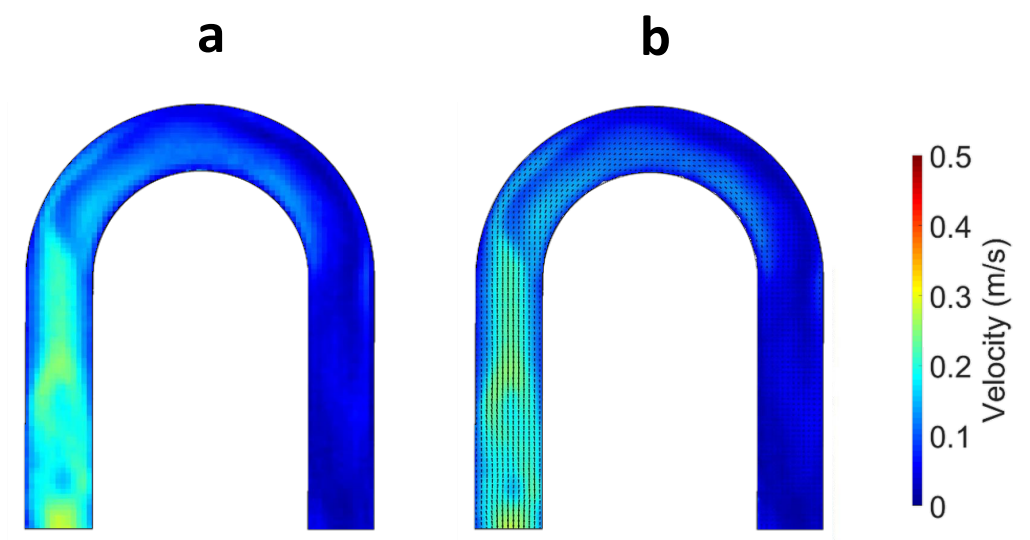

Figure 4.12: Post processed 4D Flow CMR data in the coronal plane (middle plane of the U bend) at the time instant 2: (a) velocity map and (b) velocity vectors overlaid on the velocity contour plot.

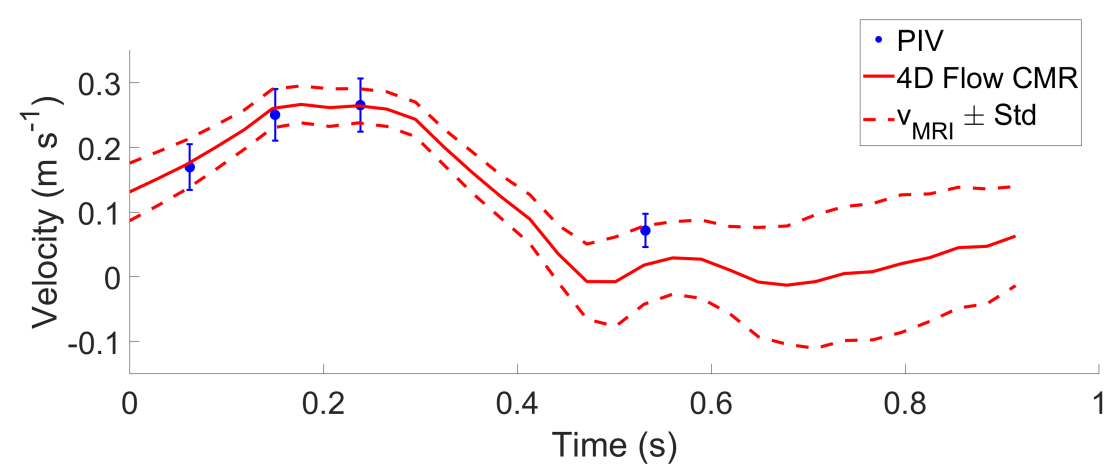

Figure 4.13: Average velocity at the inlet of the U bend during the pump cycle measured with 4D Flow CMRI (with dashed line representing the average velocity ) and PIV (with standard deviation, at the four instants of the cycle considered for the comparison). 


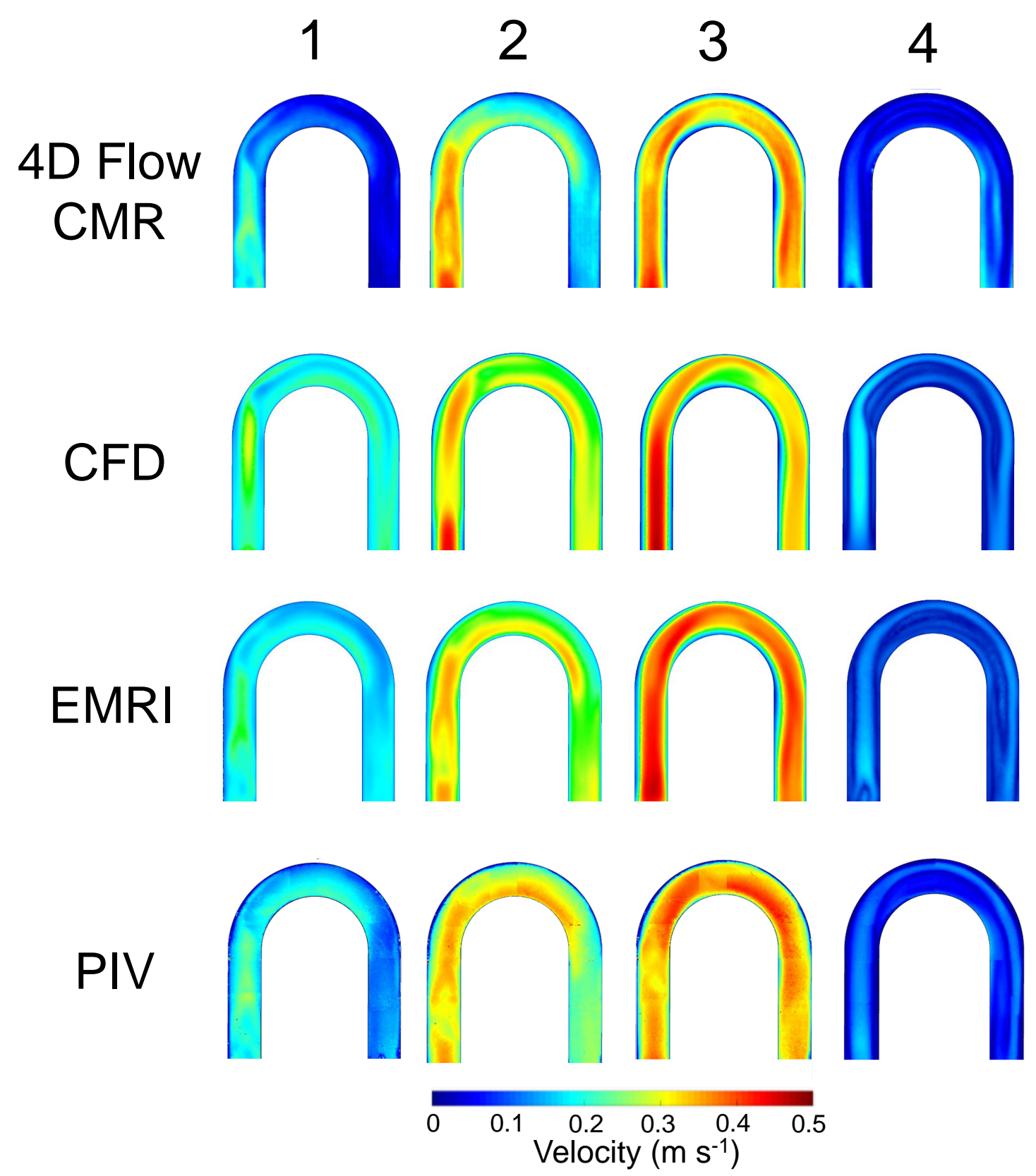

Figure 4.14: Velocity maps in the selected plane at the 4 instant measured with 4D Flow CMRI and PIV and computed with CFD and EMRI. 


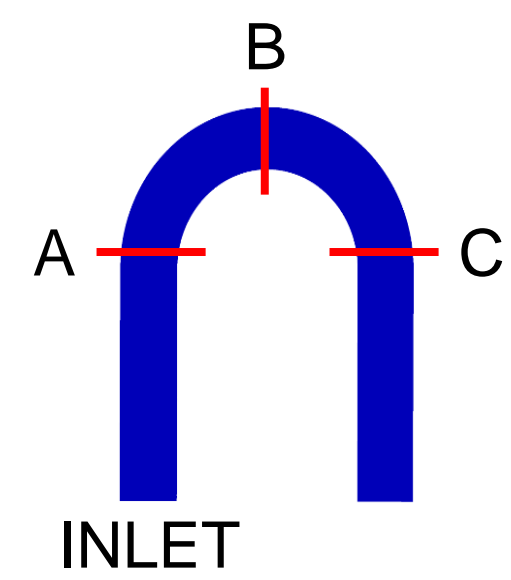

Figure 4.15: Three sections of the U bend where the velocity profiles are compared. 


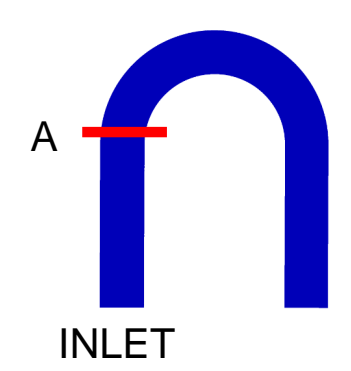

1

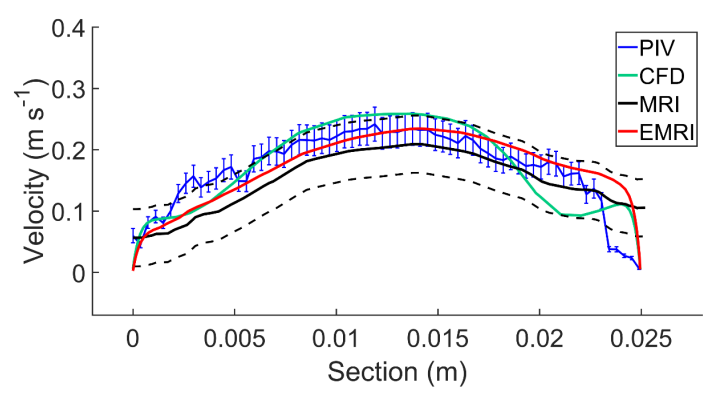

3

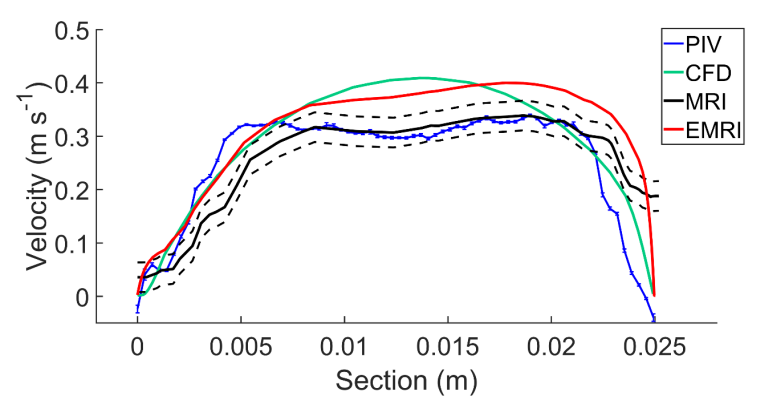

2

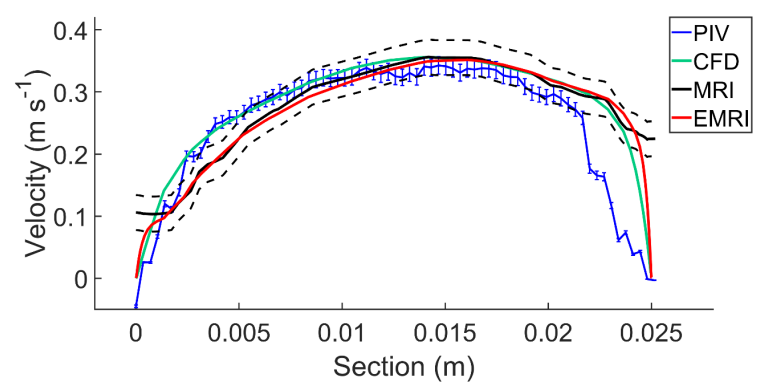

4

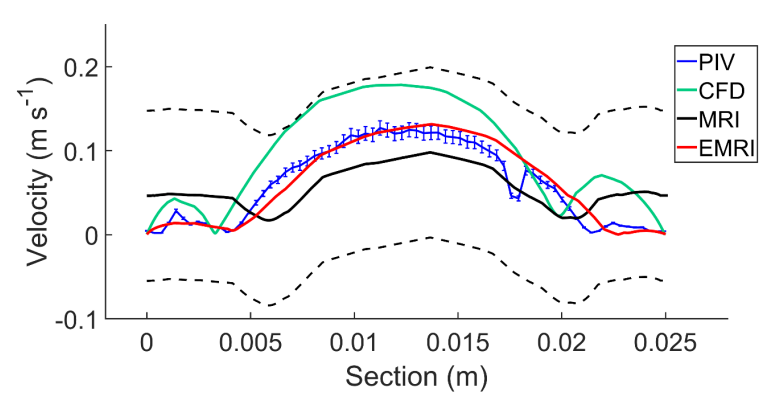

Figure 4.16: Velocity profiles in section $A$ at the 4 instant of time considered, measured with 4D Flow CMRI and PIV (with standard deviation) and computed with CFD and EMRI. 


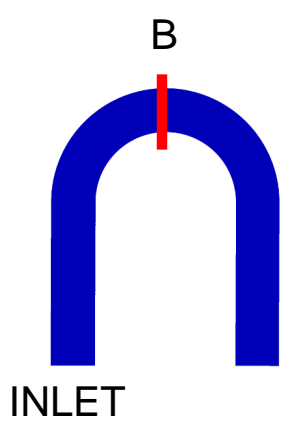

1

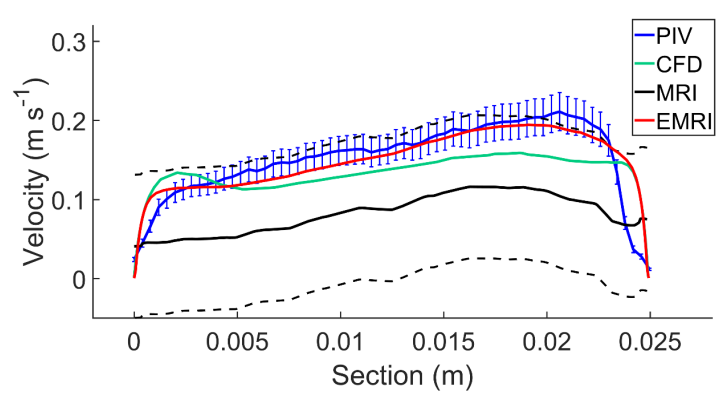

3

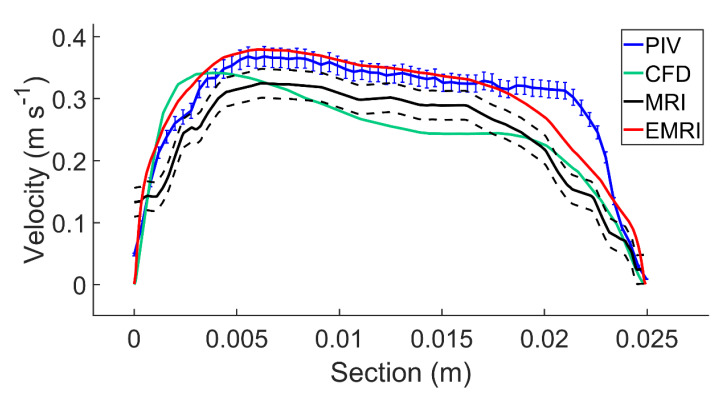

2

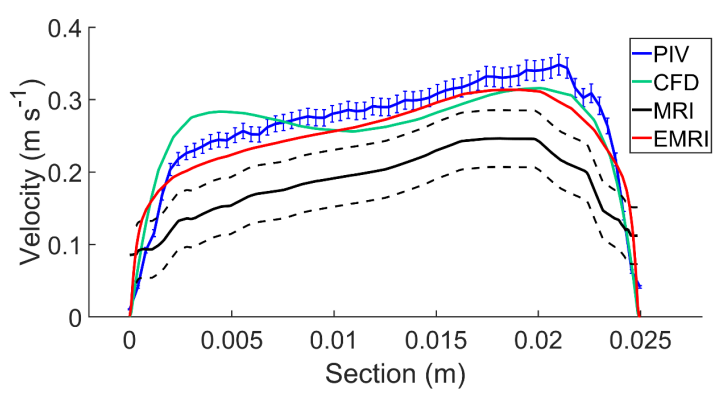

4

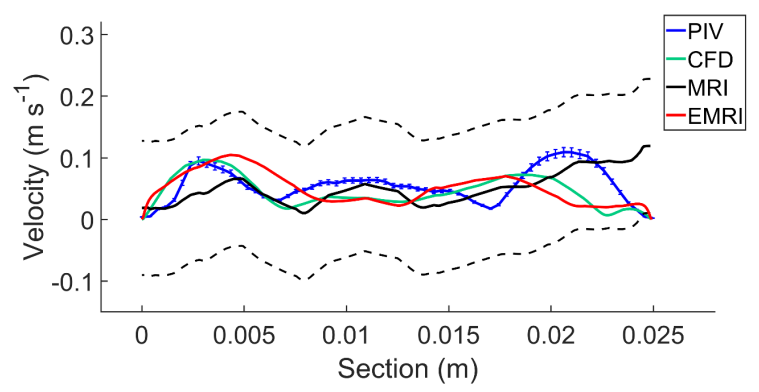

Figure 4.17: Velocity profiles in section $B$ at the 4 instant of time considered, measured with 4D Flow CMRI and PIV (with standard deviation) and computed with CFD and EMRI. 


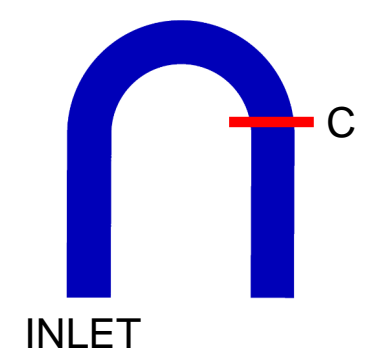

1

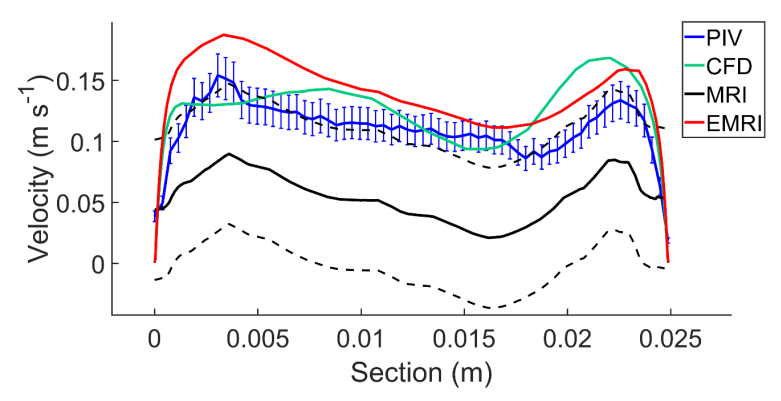

3

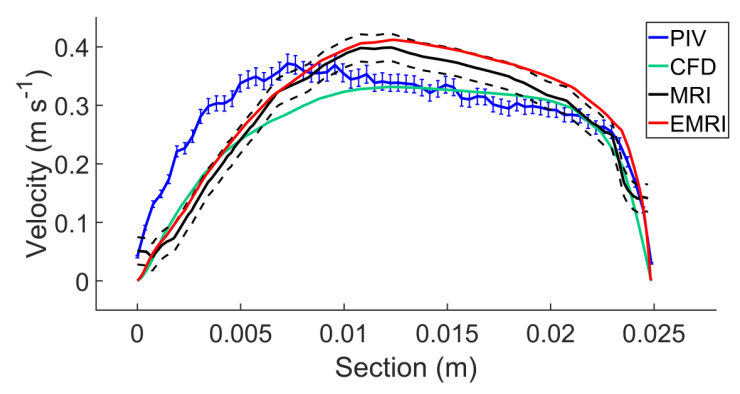

2

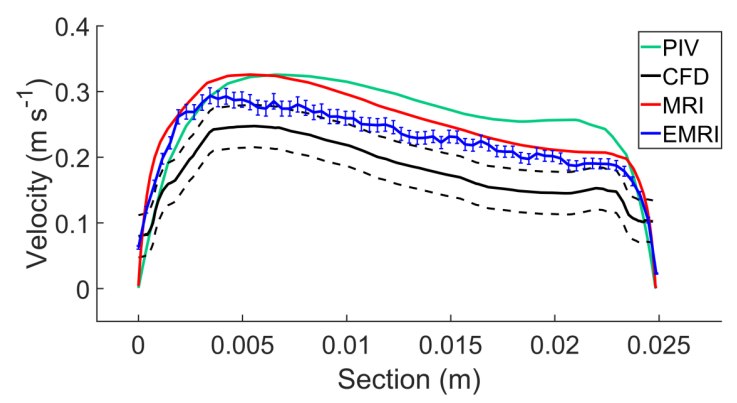

4

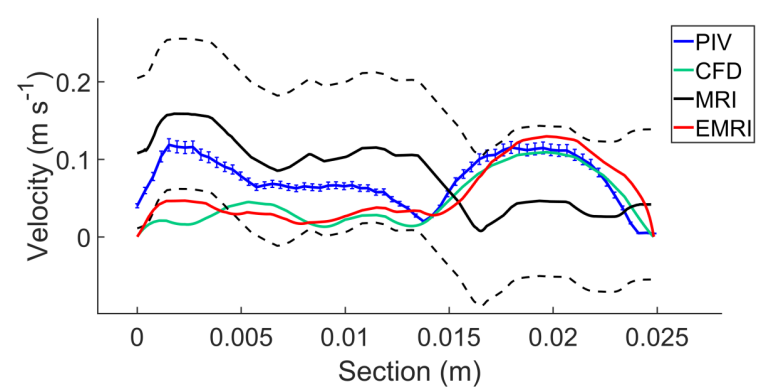

Figure 4.18: Velocity profiles in section $\mathrm{C}$ at the 4 instant of time considered, measured with 4D Flow CMRI and PIV (with standard deviation) and computed with CFD and EMRI. 


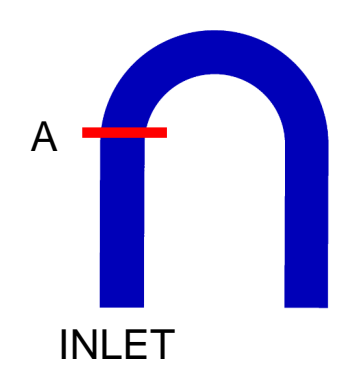

1

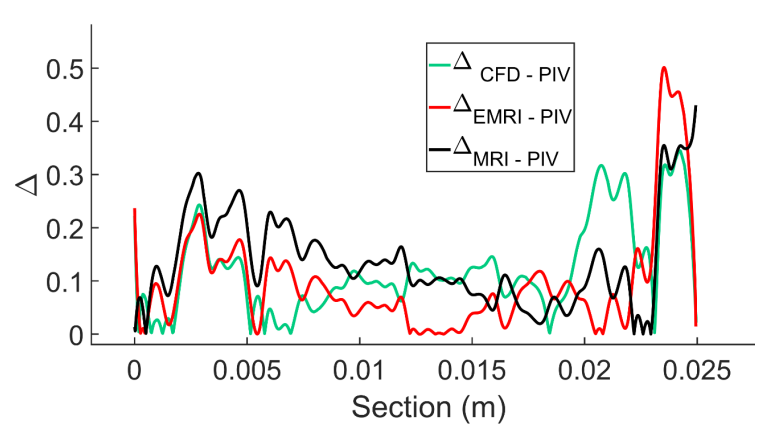

3

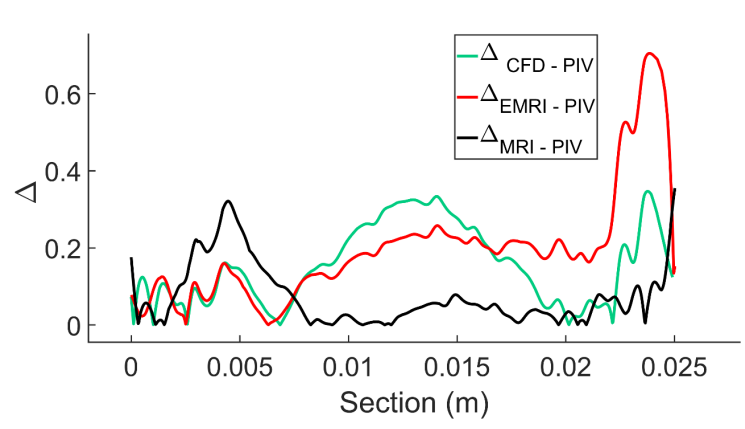

2

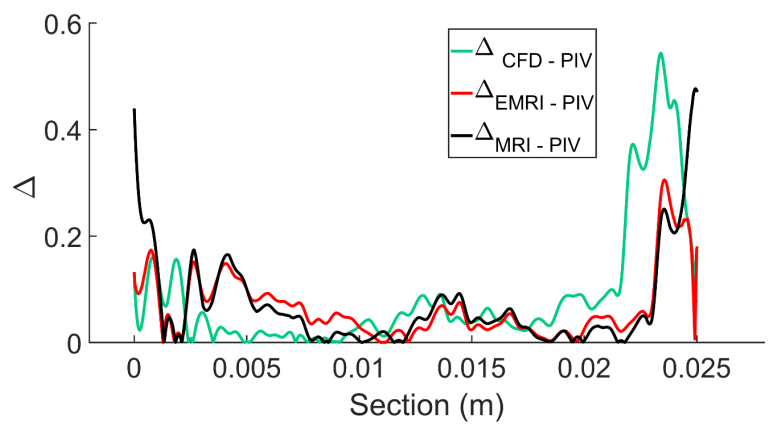

4

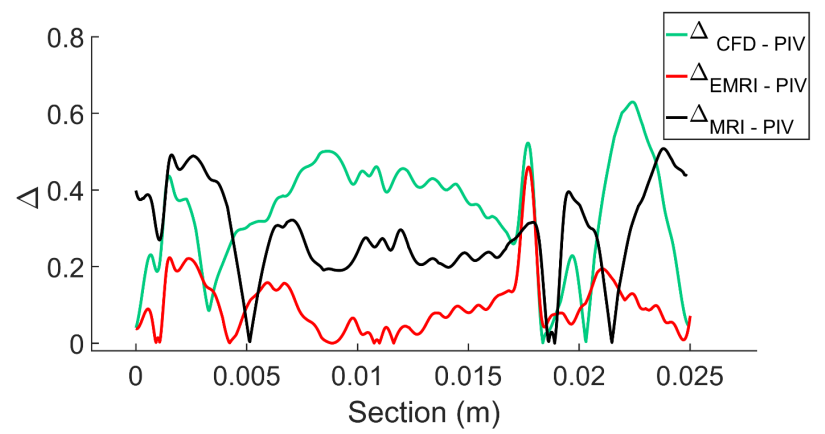

Figure 4.19: Relative difference between PIV and CFD, and PIV and EMRI velocity magnitudes $(v)$ in section A at the 4 instant of time considered. 


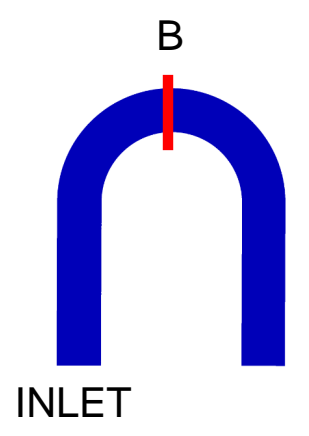

1

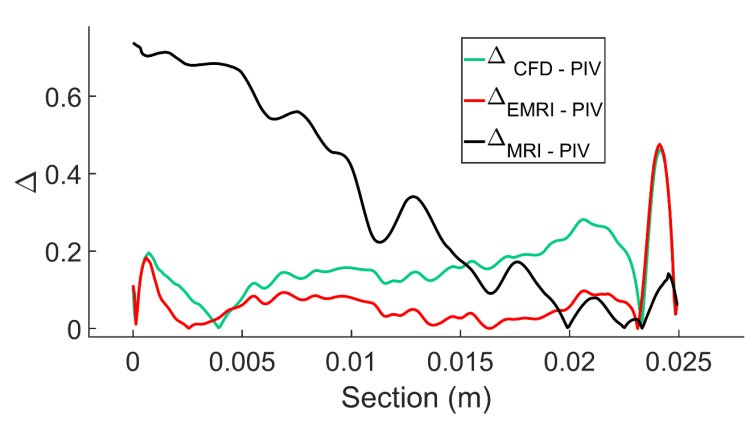

3

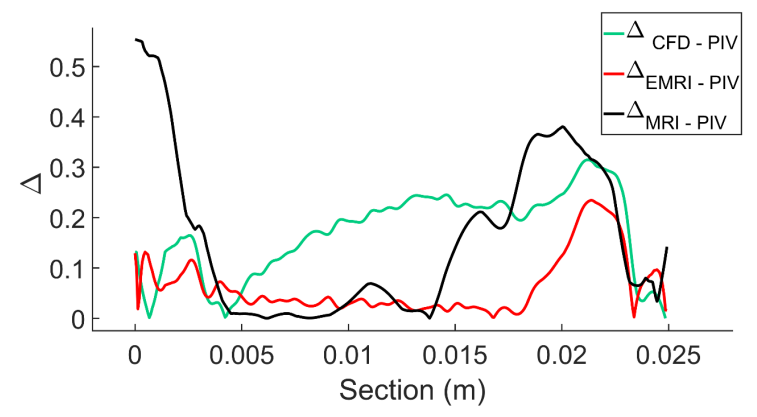

2

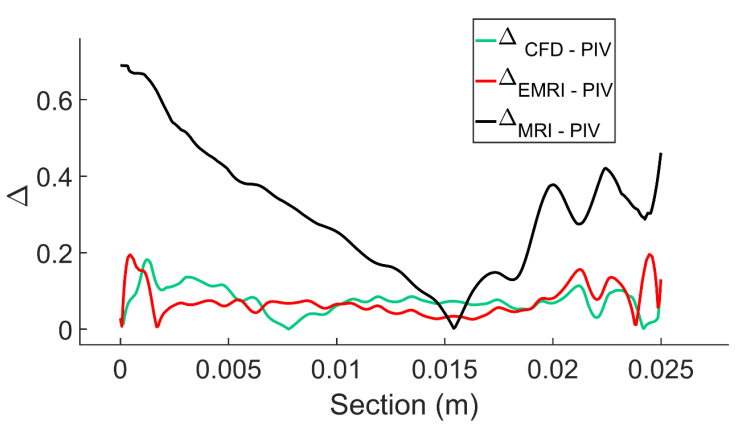

4

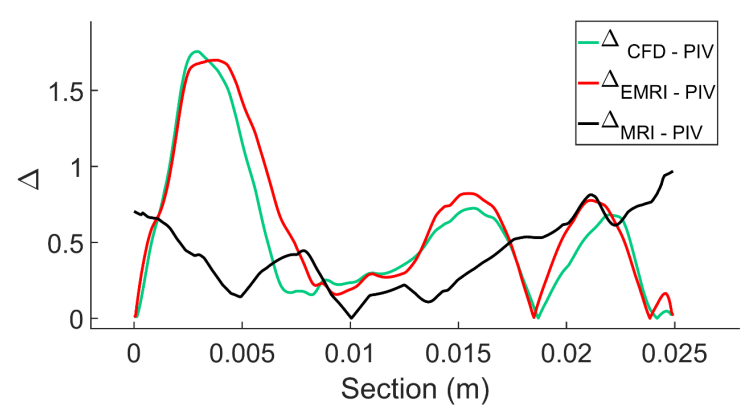

Figure 4.20: Relative difference between PIV and CFD, and PIV and EMRI velocity magnitudes $(v)$ in section $\mathrm{B}$ at the 4 instant of time considered. 


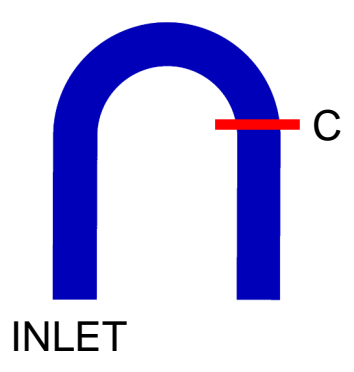

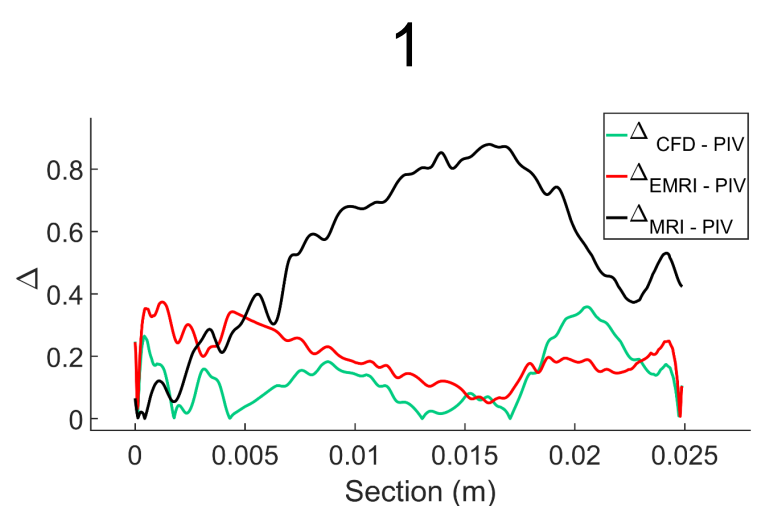

3

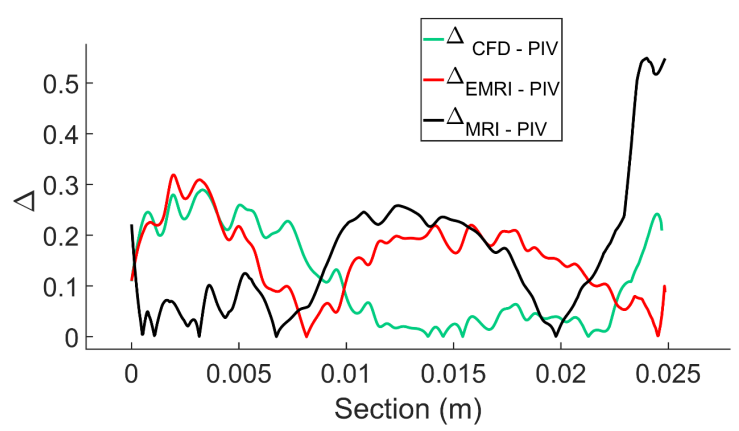

2

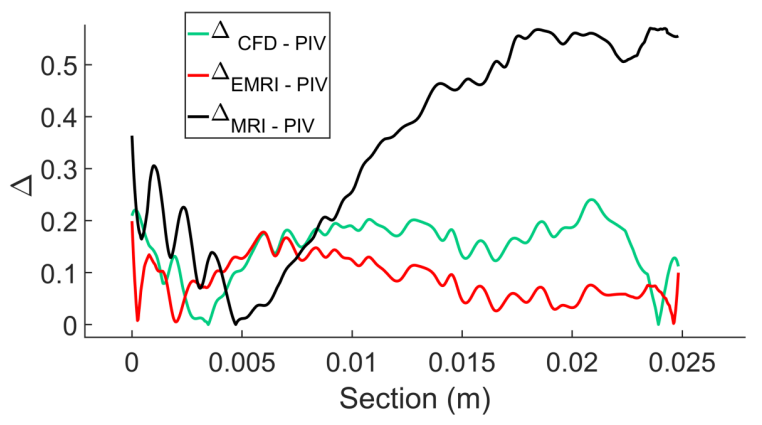

4

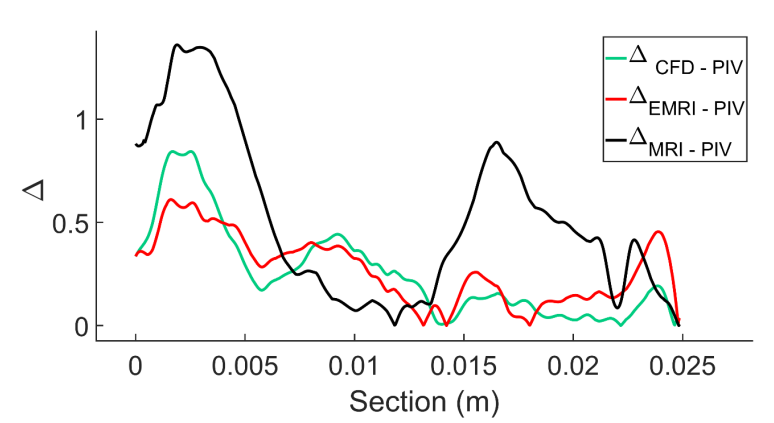

Figure 4.21: Relative difference between PIV and CFD, and PIV and EMRI velocity magnitudes $(v)$ in section $\mathrm{C}$ at the 4 instant of time considered. 


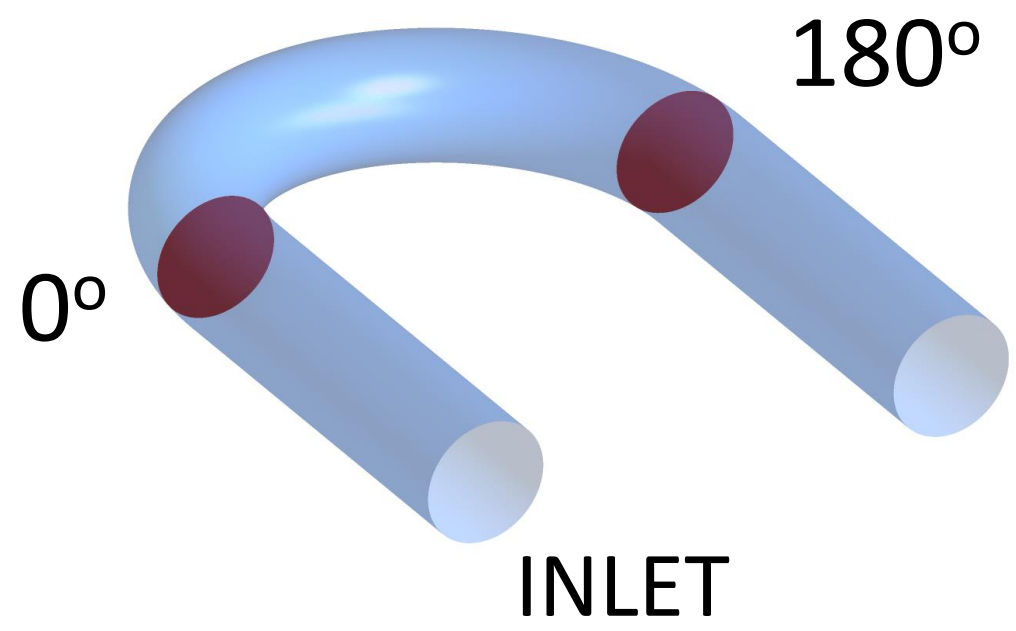

Figure 4.22: Cross sections of the $\mathrm{U}$ bend at $0^{\circ}$ and $180^{\circ}$. 


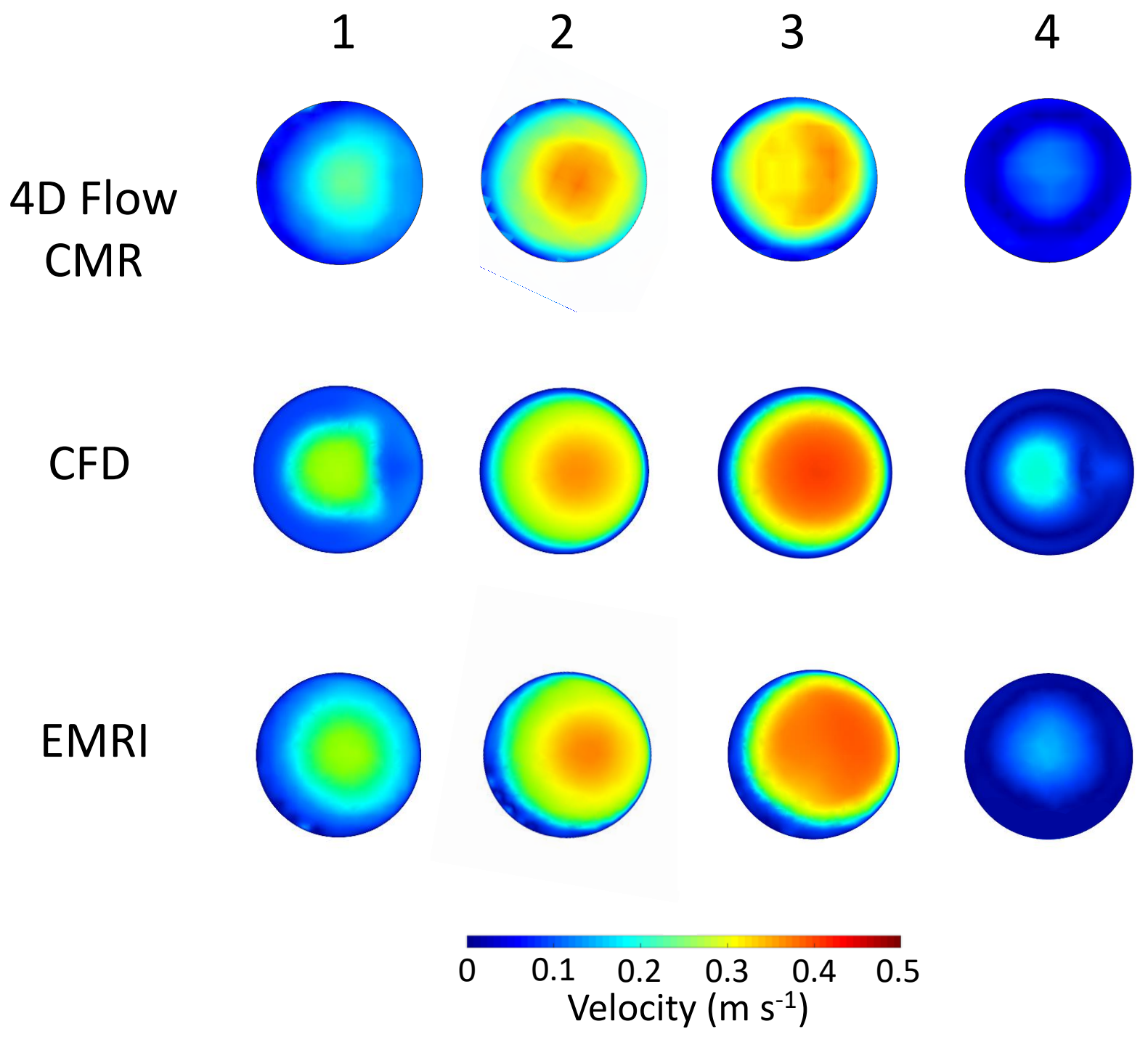

Figure 4.23: Velocity maps in the cross sectional plane at $0^{\circ}$ at the 4 instants measured with 4D Flow CMRI and computed with CFD and EMRI. 


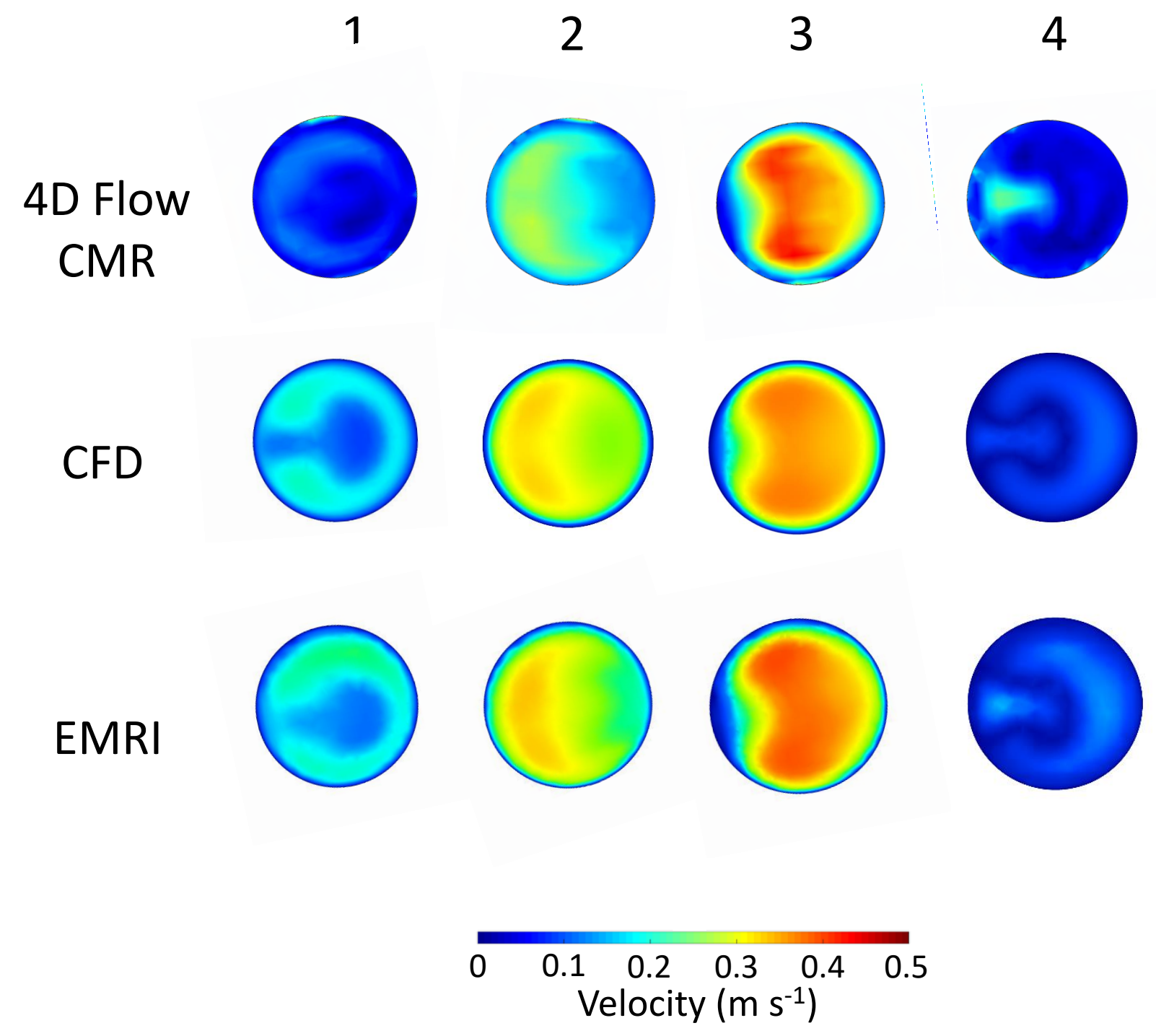

Figure 4.24: Velocity maps in the cross sectional plane at $180^{\circ}$ at the 4 instants measured with 4D Flow CMRI and computed with CFD and EMRI. 


\section{Chapter 5}

\section{D EMRI: patient-specific}

\section{application}

In the previous chapter the EMRI approach was validated against $2 \mathrm{D}$ PIV in a U bend, showing the capability of EMRI of correcting for the inaccuracies of 4D Flow CMR and capturing flow features that a CFD model might not correctly reproduce as a consequence of modelling assumptions and simplifications. In this chapter the EMRI methodology is applied to study the flow in a patient specific aorta.

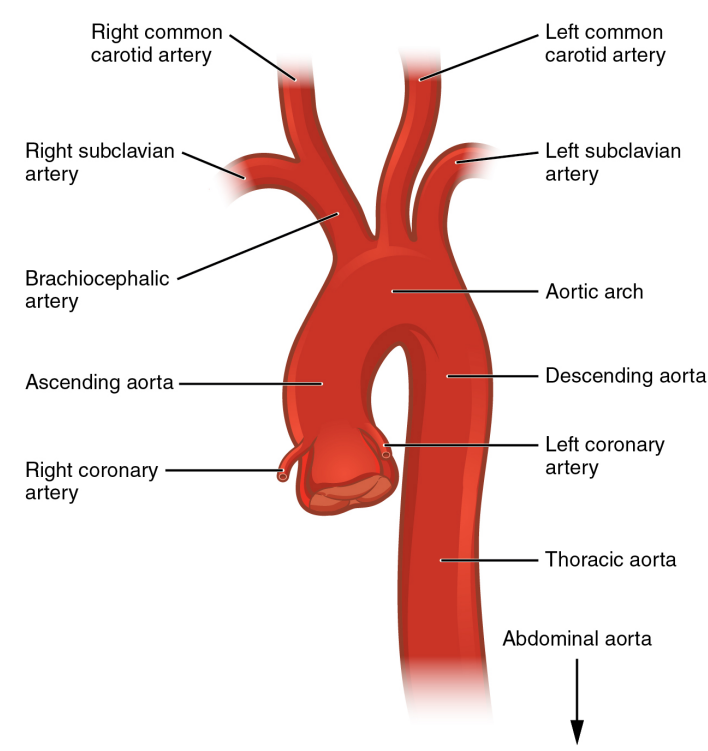

Figure 5.1: A model of the human aorta.

The aorta is the largest artery of the human body (Figure 5.1), which consists of ascending aorta, aortic arch and descending aorta. This vessel is charac- 
terized by numerous branches, and the main ones are branching out from the aortic arch, in particular the brachio-cephalic artery (BCA), the left common carotid artery (LCCA) and the left sub-clavian artery (LSCA).

Together with coronary and peripheral artery diseases, aortic diseases such as atherosclerosis, aortic aneurysms, acute aortic syndromes (e.g. aortic dissection) contribute importantly to the spectrum of cardiovascular diseases [111].

Endothelial cells, forming the innermost layer of the arterial walls, are directly exposed to the blood flow, and the fluid drag force acting on the vessel walls via mechano-transduction, results in biochemical signals, which induce changes in cells behaviour and vascular morphology [112]. Therefore flow separation, secondary flow, and wall shear stress are haemodynamic factors which play a big role in endothelial dysfunction and arterial wall remodelling, which are directly linked to the development of CVDs.

The blood flow entering the aortic arch is skewed to the inner wall during the acceleration phase, while during the deceleration, the flow direction is reversed. As a consequence this region is exposed to high and low wall shear stress which are thought to correlate with development and progress of CVDs such as atherosclerotic lesions or aneurysms. As a consequence there is a huge interest in investigating the flow patterns in the aorta in order to gain more insights into the pato-physiological processes at the base of CVDs [113].

As mentioned blood flow information could be obtained by means of 4D Flow CMR or modelled with CFD. As explained in the previous chapters 4D Flow CMR allows non invasive in vivo acquisition of velocity fields, however the limited spatial and temporal resolutions, and the signal to noise ratio, result in considerable underestimation of cardiovascular biomarkers, such as WSS [114]. In fact conventional MRI acquisitions are characterized by a single value of $v_{E N C}$, hence introducing a bias of the SNR toward the assessment of velocities in the $v_{E N C}$ range.

Aortic flow constitutes a good example, as the $90 \%$ of the blood flow velocity is less than $0.2 \mathrm{~ms}^{-1}$ in late diastole, and with with a $v_{E N C}$ of $150 \mathrm{~ms}^{-1}$ these velocities are acquired with a SNR 10 times lower than if a suitable value of $v_{E N C}$ 
were used [115]. The same issue affects the velocities in proximity of the walls, where limited spatial resolution and partial volume effects, make the estimation of velocity-dependent parameters challenging [9].

The development of CFD has allowed to investigate haemodynamics, in particular thanks to computational models' ability to quantify variables difficult-tomeasure in-vivo, and to give access to very high resolution. The limitations of CFD are mainly linked to model assumptions, such as the choice of blood rheological model, the interaction of blood with vessel walls and the boundary conditions, as discussed in the previous chapters.

The aim of this chapter is to apply the EMRI to study the blood flow in the human aorta, and in particular:

- to analyse the EMRI computed bio-mechanical parameters in a patient specific geometry.

- to compare the flow and the flow-derived parameters (e.g. WSS) computed with EMRI and CFD in the aorta during the cardiac cycle.

Part of the results presented in this chapter have been published in the ASME Journal of Engineering and Science in Medical Diagnostics and Therapy [3] and will be presented at the Virtual Physiological Human (VPH) Conference 2020.

\subsection{Methods}

\subsubsection{Magnetic resonance imaging and post processing}

The data set used was the same as chapter 3.3. Let us recall the main parameters of sequence used: TR $4.4369 \mathrm{~ms}$, TE $2.6335 \mathrm{~ms}$, flip angle $15^{\circ}$, matrix 256256 , pixel dimension $1.36 \times 1.36 \times 3 \mathrm{~mm}^{3}$, number of slices 17 , number of phases 20 . The velocity encoding was made in three orthogonal directions: head-foot, right-left and anterior-posterior with $v_{E N C}$ of $130 \mathrm{~cm} \mathrm{~s}^{-1}$. The image sets were acquired at 20 equally-spaced intervals over the cardiac cycle, collecting 120 volumetric image sets (20 time points x 3 encoding directions x pairs of magnitude and phase images). 
The anatomy of the aorta was segmented from magnitude images, averaging the three encoding directions, using Simpleware ScanIP (version O-2018.12-SP2, Synopsys, CA, USA) and meshed using ICEM CFD (ANSYS Inc, Cannonsburg, PA, USA). The geometry (Figure 5.2) was meshed using tetrahedral elements and prism elements for the boundary layer (10 layers) near the wall. The mesh, that was chosen after a sensitivity test (see A.2), has 433765 nodes and 1396493 elements.

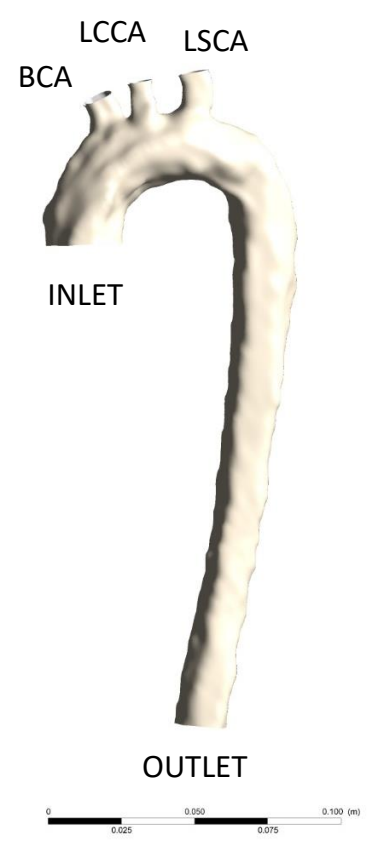

Figure 5.2: 3D geometry of the aorta.

4D Flow CMR data were first processed using an in-house code written in Matlab environment (Matlab, MathWorks, Natick, MA), that extracts the velocity components at the inlet of the aortic root, at the brachio-cephalic artery (BCA), at the left common carotid artery (LCCA) and at the left sub-clavian artery (LSCA) and also the velocity field in the entire aortic domain. The average velocity at the inlet, at the BCA, LCCA and LSCA outlets during the heart cycle are presented in Figure 5.3.

The flow regime is characterize by a Womersley number $(\alpha)$ of 18.87 , a peak Reynolds number $R e_{\text {peak }}$ of 2905.6 and an average Reynolds number $R e_{\text {mean }}$ of 


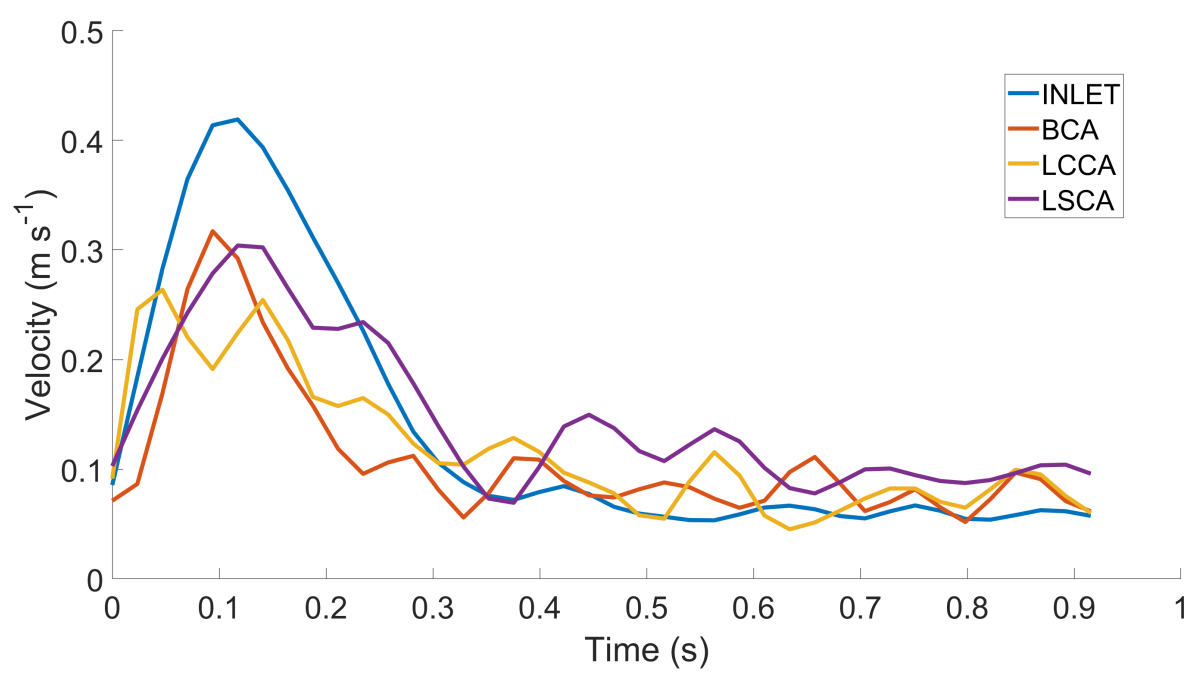

Figure 5.3: Average velocity at the inlet of the aorta, at BCA, LCCA and LSCA during the cardiac cycle.

976.8. To assess the onset of turbulence, the critical Reynolds number $R e_{c r i t}$ was computed with (Equation 4.9):

$$
\operatorname{Re}_{c r i t}=169 \alpha^{0.83} S t^{-0.27}
$$

where $S t$ is Strouhal number, calculated as (Equation 4.10):

$$
S t=\frac{D f}{2\left(v_{\text {peak }}-v_{\text {mean }}\right)}
$$

where $D$ is the aortic diameter $(0.02726 \mathrm{~m}), f$ is the frequency of the cardiac cycle, $v_{\text {peak }}$ and $v_{\text {mean }}$ are respectively the peak and the mean velocity over the cardiac cycle. In the patient specific aorta studied the $R e_{c r i t}=4160$, therefore the flow regime could be considered laminar [116].

The accuracy of the velocity measured with MRI was assessed by computing the standard deviation as in section 4.1.3, using equation 5.3 [101]:

$$
\sigma_{v}=\frac{\sqrt{2} \cdot V_{E N C}}{\pi S N R}
$$

where SNR is the signal-to-noise ratio of the magnitude images, calculated as the ratio of the average pixel intensity in two equally sized regions of interest, one 
in the aortic lumen and the other in stationary tissue. The standard deviation was assessed throughout the cardiac cycle, and it is shown in Figure 5.4 together with the inlet velocity waveform.

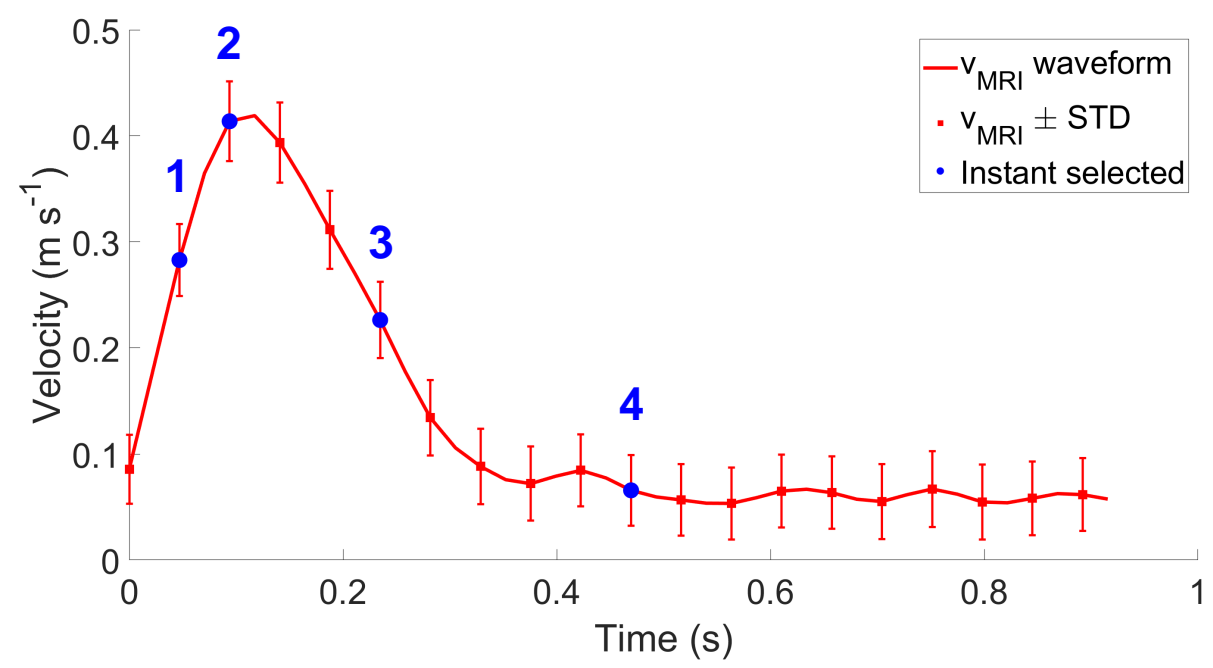

Figure 5.4: Average velocity at the inlet of the aorta with standard deviation and the instants selected for the comparison of 4D Flow CMR, CFD and EMRI (blue dots).

Another important parameter to assess the quality of the velocity mapping is the $V N R$ that is defined as $4.6[101]$ :

$$
V N R=\frac{v}{\sigma_{v}}
$$

where $v$ is the measured velocity. As pointed out previously, the $v_{E N C}$ is set to be higher than the peak systole velocity, which impacts negatively the VNR during diastole. This is evident by the values in Table 5.1, where the SNR, the VNR and the $\sigma_{v}$ of the 4D Flow CMR of the 4 instants of the cardiac cycle considered for the comparison between 4D Flow CMR, CFD and EMRI, are presented.

In order to estimate the WSS from the 4D Flow MRI data, the retrieved flow field was interpolated on the computational mesh, requiring additionally the velocity on the wall to be zero $[63,9]$. In order to do so $v_{M R I}$ was defined as follows (Equation 5.5): 
Table 5.1: SNR,VNR and $\sigma_{v}$ of the 4D Flow CMR data at the 4 instants considered of the cardiac cycle.

\begin{tabular}{|c|c|c|c|}
\hline \hline Instant & SNR & VNR & $\sigma_{v}\left(m s^{-1}\right)$ \\
\hline 1 & 14.11 & 8.36 & 0.034 \\
2 & 12.73 & 11.02 & 0.037 \\
3 & 13.28 & 6.29 & 0.036 \\
4 & 14.30 & 1.97 & 0.033 \\
\hline \hline
\end{tabular}

$$
v_{M R I}=\left\{\begin{array}{lll}
v_{M R I} \cdot \frac{1}{1.5 m m}\left(d_{W A L L}\right) & \forall & d_{W A L L}<1.5 \mathrm{~mm} \\
v_{M R I} & \forall & d_{W A L L}>1.5 \mathrm{~mm}
\end{array}\right.
$$

so that $v_{M R I}$ within half voxel distance $(1.5 \mathrm{~mm})$ from the aortic wall along the normal of the aortic surface would smoothly approach zero on the wall [12]. This velocity field was used for the WSS computation only, whereas the raw 4D Flow CMR measured velocity field was used in the EMRI approach.

In Figure 5.5 the raw 4D Flow CMR velocity profile and the processed one for computing the WSS are shown in a line crossing the descending aorta.
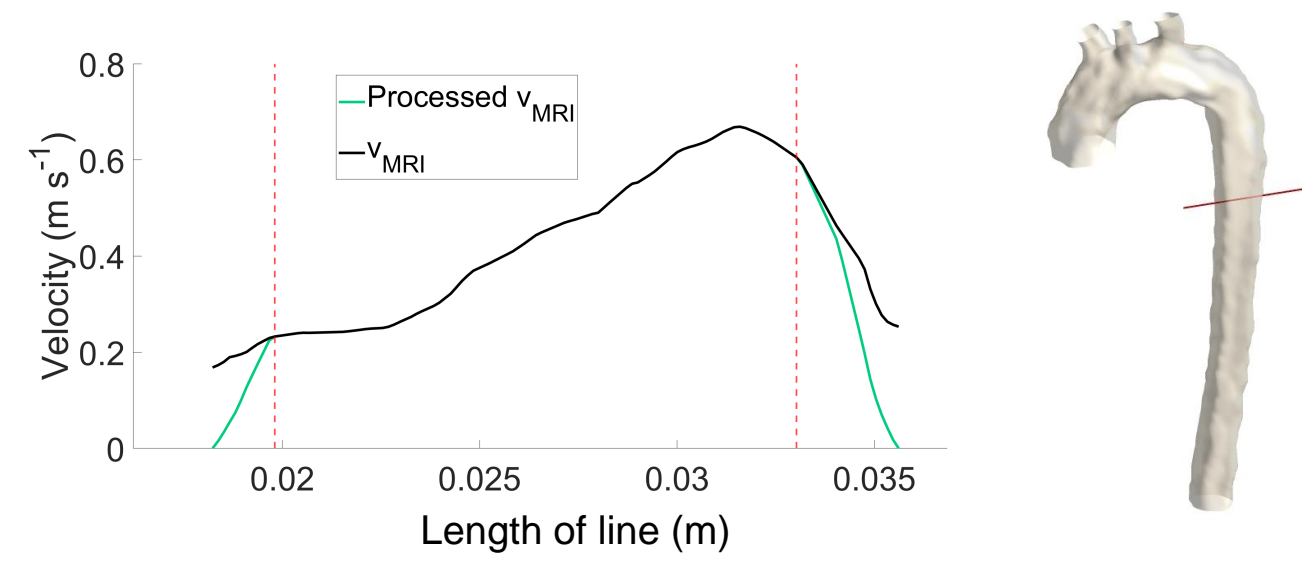

Figure 5.5: Raw 4D Flow CMR velocity profile and processed one at $t=0.2348 \mathrm{~s}$ from the beginning of the systole, computed on the line crossing the descending aorta (on the right of the picture).

In Figure 5.6 the velocity surfaces of the raw 4D Flow CMR velocity field and the processed one in a transverse plane of the descending aorta (Figure $5.10 \mathrm{~b}$ ) are 
showed.

\section{D Flow CMR}

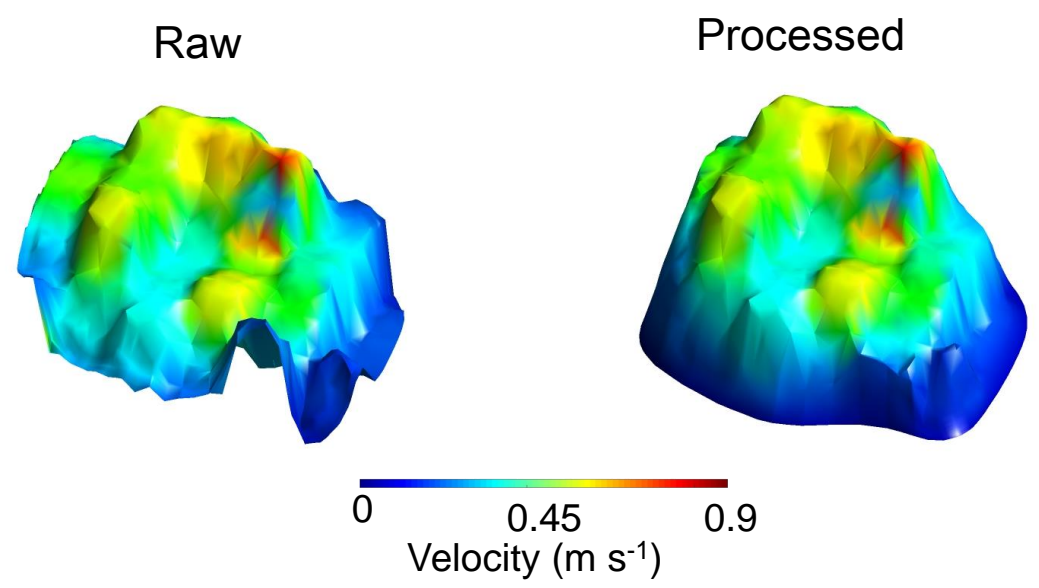

Figure 5.6: Velocity surfaces computed from raw 4D Flow CMR velocity profile and processed one at $\mathrm{t}=0.2348 \mathrm{~s}$ from the beginning of the systole, in a transverse plane of the descending aorta (shown Figure $5.10 \mathrm{~b}$ ).

\subsubsection{Enhanced Magnetic Resonance Imaging}

\subsubsection{EMRI Wall function}

As it was pointed out previously, WSS is one of the main cardiovascular biomarker. It is related to the gradient of the velocity field near the wall. When considering 4D Flow CMR data, it is needed to take into consideration that the spatial resolution is defined by the size of the voxels of the image (usually $1-2 \mathrm{~mm}^{3}$ ). This is true also near the wall.

Figure 5.7 shows the raw velocities measured with 4D Flow CMR in a transversal cross section of a patient specific aorta, with the segmentation profile of the vessel overlaid. It is straightforward to notice how the segmentation line intersects the voxels on the boundary layer, hence in this region the velocities are usually represented by only one voxel.

If these facts are taken into consideration, the introduction in the EMRI framework, of a function dependent on the distance from the vessel wall $d_{W A L L}$, could be vital to alleviate the 4D Flow CMR spatial resolution limitations, especially in real 

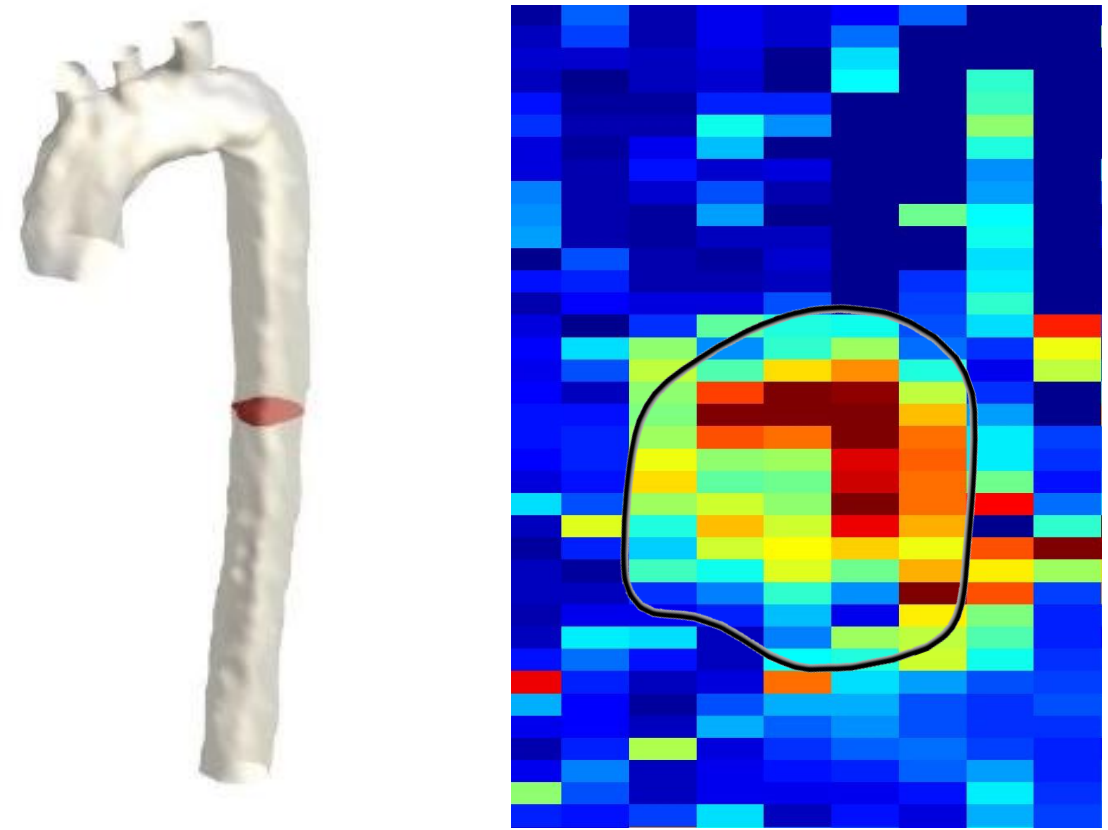

Figure 5.7: Diagram showing the raw velocity measured with 4D Flow CMR (right end side) in a transversal cross section of a patient specific aorta (left hand side), with the segmentation profile of the vessel overlapped.

case scenarios in which an estimation of parameters dependent on the velocity field in proximity of the walls, such as WSS, is needed.

In particular the function $\phi\left(d_{W A L L}\right)$ was introduce to ensure a smooth transition of the $v_{M R I}$ from 0 when moving radially from the wall towards the vessel centreline, so that:

- if $d_{W A L L}$ is the distance from the aortic wall, $\phi\left(d_{W A L L}=0\right) \approx 0$

- if $\Delta$ Pix is the chosen offset from the wall, $\phi(\Delta P i x) \approx 1$

The resulting function for $\phi\left(d_{W A L L}\right)$ could be written in a linear form in the following way (Equation 5.6):

$$
\phi\left(d_{W A L L}\right)=\left\{\begin{array}{lll}
\frac{1}{\Delta P i x}\left(d_{W A L L}\right) & \forall & d_{W A L L}<\Delta P i x \\
1 & \forall & d_{W A L L}>\Delta P i x
\end{array}\right.
$$

The EMRI force is therefore expressed as (5.7):

$$
\vec{f}=K \cdot\left(\phi\left(d_{W A L L}\right) \cdot \vec{v}_{\exp }-\vec{v}\right)
$$


In order to find the optimal $\Delta$ Pix a sensitivity test was performed, computing the following parameters: kinetic energy (Equation 3.7), $\varepsilon v_{T O T}$ and $\varepsilon v_{B}$ which represent the difference between the computational velocity $v$ and the $v_{M R I}$ averaged over the computational domain and averaged on the volume formed between the wall and a surface translated normally inward with an offset of $1.5 \mathrm{~mm}$ respectively. This approach will be referred as EMRI Wall function and it will be compared with the standard EMRI.

\subsubsection{EMRI computational models}

In the EMRI approach the flow was assumed to be laminar and incompressible. The blood was assumed Newtonian, with its density and viscosity set to $1060 \mathrm{kgm}^{-3}$ and $0.004 \mathrm{~Pa} \cdot s$ [12]. Walls were assumed to be rigid and a no-slip boundary condition was applied. The EMRI algorithm was run in steady state using the Cartesian velocity profile at the inlet, BCA, LCCA, LSCA . A $0 P a$ boundary condition was applied at the outlet of the descending aorta (Figure 5.2).

In order to have a force coefficient $k$ ranging from 0 to 1 in the domain, as in all the other cases studied, a scaling factor $s$ had to be applied (Equation 3.10), taking into consideration the characteristic length (the aortic diameter $L=0.02726 \mathrm{~m}$ ), the characteristic velocity (refer to Figure 5.4) of the aorta, and the value of the fluid density. The values of $s$ are reported in Table 5.2.

Table 5.2: Gain scaling $s$ at the considered instants of the heart cycle.

\begin{tabular}{|c|c|}
\hline \hline Instant & $s$ \\
\hline 1 & 7.922 \\
2 & 5.497 \\
3 & 9.733 \\
4 & 29.404 \\
\hline \hline
\end{tabular}

\subsubsection{CFD}

A transient CFD simulation of the aorta was run as comparison, with the same assumptions as the EMRI model. A time dependent velocity profile was imposed at the inlet. In order to do so the inlet of the aorta was extended (for 2 times its diameter) to apply a flat velocity profile (Figure 5.8), consistent to the measured 


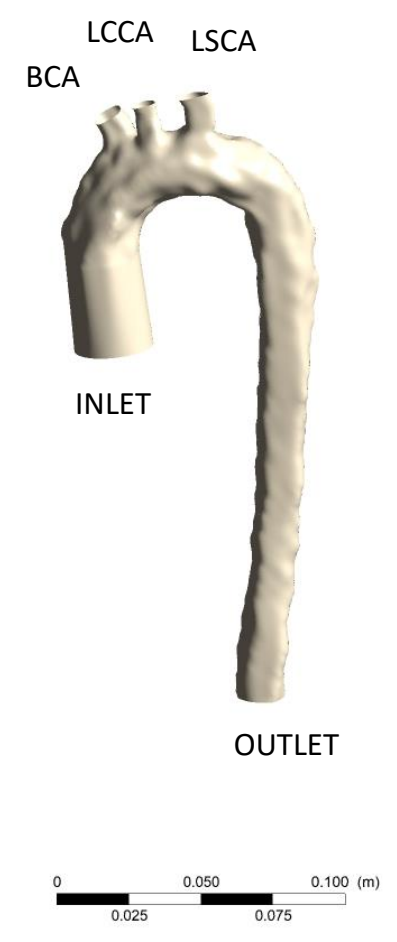

Figure 5.8: Geometry of the aorta used for the CFD simulation.

velocity waveform (shown in Figure 5.4), which was interpolated with a spline. Mass flow rate boundary condition was applied at the BCA, LCCA, LSCA based on the waveform in Figure 5.3. A $0 \mathrm{~Pa}$ boundary condition was applied at the outlet of the descending aorta (Figure 5.8).

\subsubsection{Sets of simulations conducted}

Three sets of simulations were conducted:

1. the first one analysed the whole aorta in late systole (at the time $t=0.2348$ $s$ from the beginning of systole) (instant 3 in Figure 5.4) comparing the outcome of 4D Flow CMR, steady state CFD (EMRI with k=0) and EMRI.

2. the second one aimed at comparing the outcome of EMRI and EMRI Wall function, and the impact of the introduction of a wall function on the energy and on the velocity difference between 4D Flow CMR and EMRI.

3. the third set of simulations aimed at comparing the aortic flow measured with 4D Flow CMR, computed with EMRI wall function and with time resolved 
CFD in 4 phases of the cardiac cycle (accelerating phase, peak systole, decelerating phase and mid diastole). The flow path are assessed in the sections showed in Figure 5.10.

4. the last one focused on a small portion of the artery, a $0.01 \mathrm{~m}$ section immediately downstream to the aortic arch (Figure 5.9), in order to verify that EMRI leads to reliable results even when small domains unsuitable for CFD studies are considered. This application might be very useful for regions where the estimation of the boundary condition is limited.

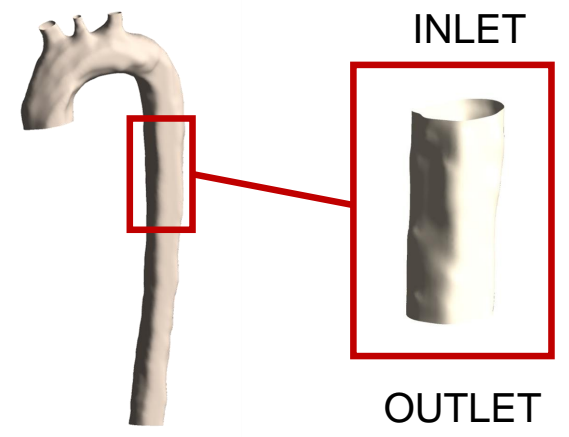

\begin{tabular}{|l|l|}
\hline Boundary & Condition applied \\
\hline Inlet & $\begin{array}{l}\text { Cartesian velocities (CFD) } \\
\text { Flat velocity (EMRI) }\end{array}$ \\
\hline Outlet & $0 \mathrm{~Pa}$ \\
\hline Walls & Rigid, Non-slip \\
\hline
\end{tabular}

Figure 5.9: Portion of the aorta geometry considered and boundary conditions applied.

\subsection{Results and discussion}

\subsubsection{Aortic flow at late systole: comparison between CFD steady state, EMRI and 4D Flow CMR flow fields.}

\subsubsection{Blood flow velocities}

The velocity profiles in the aorta, during the decelerating phase of the cardiac cycle, are shown in Figure 5.11. The profiles are shown in the mid-plane and in the descending aorta, for the original 4D Flow CMR, standard CFD and EMRI. 
a)

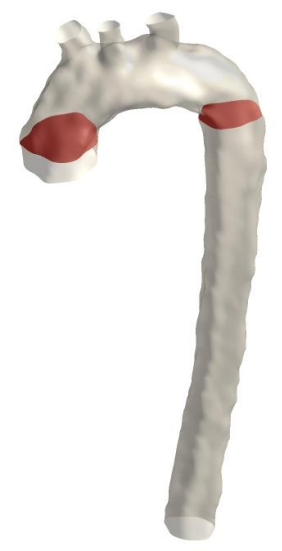

b)

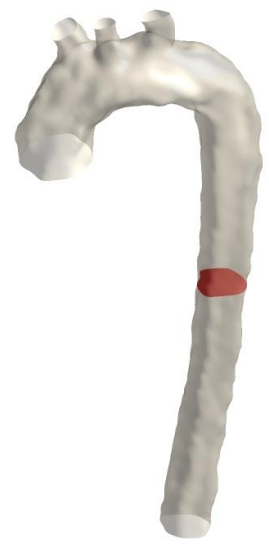

c)

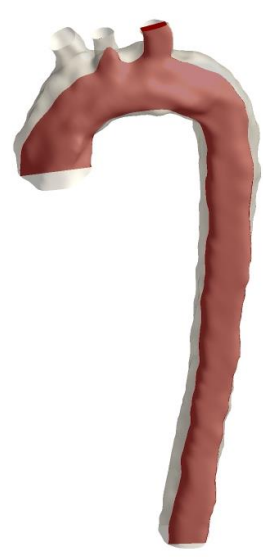

Figure 5.10: Sections considered to compare the flow path in the aorta: a) transverse sections at the inlet and outlet of the aortic arch, b) transverse section in the descending aorta and c) sagittal section of the whole aorta.

The 4D Flow CMR velocity field shows a less uniform distribution in comparison to the CFD and the EMRI. The velocities obtained in 4D Flow CMR, CFD and EMRI are generally in the same range $\left(0-0.9 \mathrm{~ms}^{-1}\right)$ which is the normal range of velocity in the aorta [117]. In the 4D Flow CMR distribution, local peaks of velocity can be observed, and the velocity pattern appears to be relatively discontinuous. Conversely, in the standard CFD, the velocity is smoothly varying.

The EMRI result reflects the spatially heterogeneous pattern of the velocity observed in 4D Flow CMR while maintaining smooth variation across the aorta. The behaviour in proximity of the wall in the EMRI is determined predominantly by the no-slip boundary condition, allowing for the correction of the non-zero velocities of the 4D Flow CMR (Figure 5.12).

A closer look at the velocity profiles, shown in Figure 5.12, indicates that the velocity near the wall in $4 \mathrm{D}$ Flow $\mathrm{CMR}$ can be as high as the velocity peak in the middle of the aorta $\left(\approx 0.9 \mathrm{~ms}^{-1}\right)$. This is likely to be noise, as even if aortic wall motion had been taken into consideration, the aortic wall would have been in the phase of gradual deflation at this time point of the cardiac cycle, i.e. end of systole. This flaw is 'corrected' in EMRI model, that shows zero velocity near the 


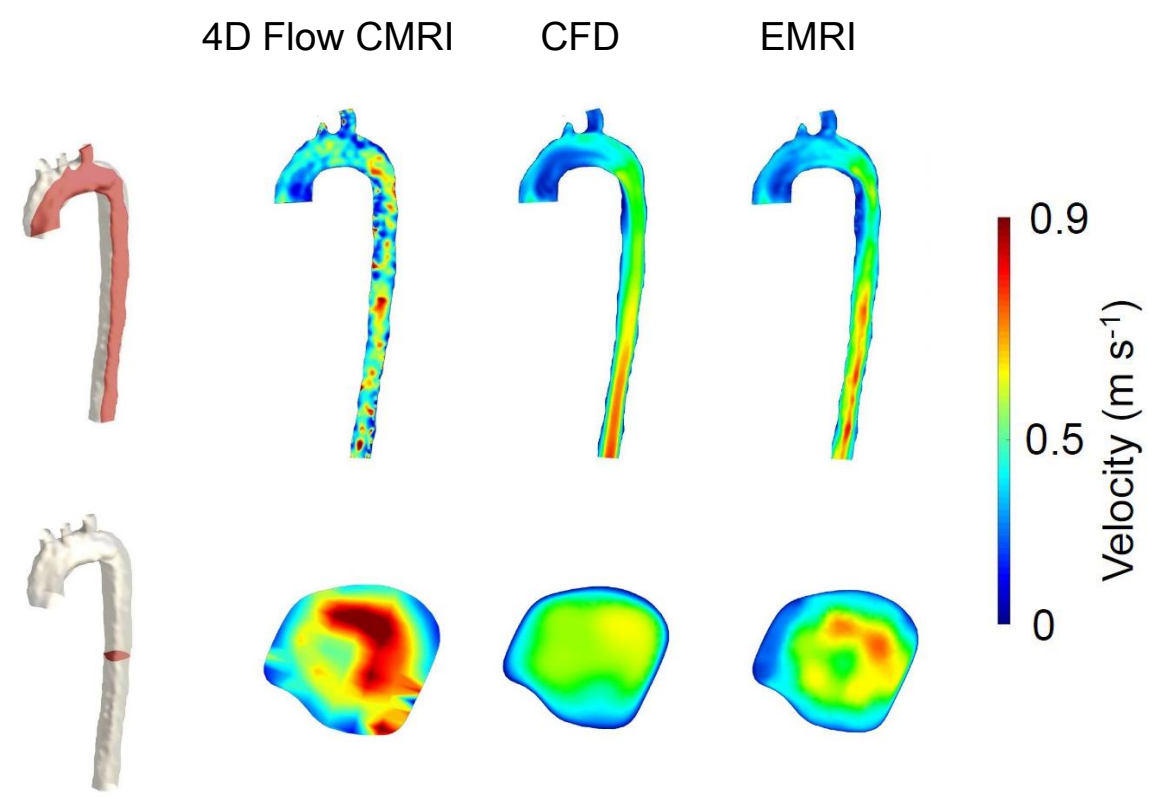

Figure 5.11: Velocity maps of the aorta $(t=0.2348 s)$ computed with 4D Flow CMR, CFD and EMRI. Sagittal cutting plane (top left) and transversal cross section (bottom left). The locations of cutting planes are shown on the left panel.

wall, because of the no-slip boundary condition imposed. This is clearly showed by the velocity difference maps between EMRI and 4D Flow CMR (Figures 5.13 and 5.14).

In the cross-sectional velocity distribution, the flow obtained by standard CFD is skewed more towards the outside of the aortic curvature (Figure 5.11), although the peak velocity is lower than the one measured by 4D Flow CMR and computed by EMRI (approximately $0.6 \mathrm{~ms}^{-1}$ in CFD vs. 0.75 and $0.9 \mathrm{~ms}^{-1}$ in EMRI and 4D Flow CMR, respectively). Here, EMRI more accurately reflects the velocity profiles measured in-vivo, including the crescent-looking pattern of high velocity.

\subsubsection{Effect of external force on kinetic energy, velocity differ- ence, pressure and WSS between 4D Flow CMR and EMRI.} In order to have a parameter to estimate how the EMRI force is changing the fluid dynamics of the system, the kinetic energy of the system, the pressure and the velocity difference between EMRI and 4D Flow CMR were computed (Table 5.3).

In particular the energy of the system changes from $1705.19 \mathrm{~J}$ of the stan- 


\section{D MRI}

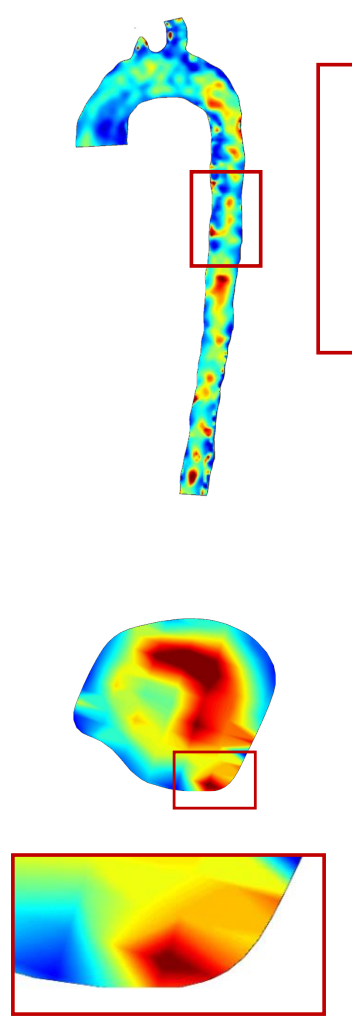

EMRI

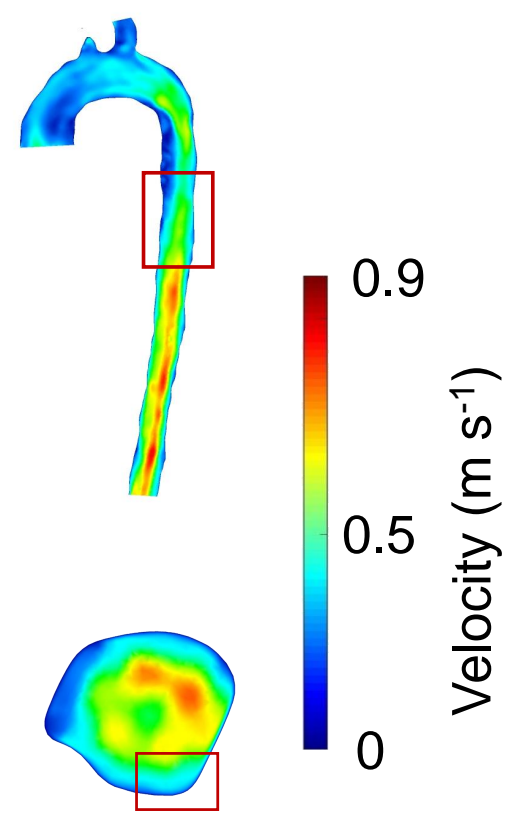

Figure 5.12: Velocity maps of the aorta $(t=0.2348 s)$ of the Sagittal cutting plane (top row) and transversal cross section (bottom row) computed with 4D Flow CMR and EMRI, with a magnification of a portion of the descending aorta and of a portion near the wall.

Table 5.3: Quantities used to assess the impact of the introduction of $\phi\left(d_{W A L L}\right)$ for different $\Delta P i x$ values.

\begin{tabular}{|c|c|c|c|c|c|}
\hline \hline$\Delta$ Pix $(\mathrm{mm})$ & $\begin{array}{c}\text { Kinetic } \\
\text { Energy }(J)\end{array}$ & $\begin{array}{c}\text { Pressure } \\
(\mathrm{Pa})\end{array}$ & $\begin{array}{c}\varepsilon v_{T O T} \\
\left(m s^{-1}\right)\end{array}$ & $\begin{array}{c}\varepsilon v_{B} \\
\left(m s^{-1}\right)\end{array}$ & Iterations \\
\hline CFD & 1702.32 & 320.912 & 0.197843 & 0.0691595 & 100 \\
EMRI & 1931.76 & 2024.99 & 0.133665 & 0.0497925 & 42 \\
\hline \hline
\end{tabular}

dard CFD simulation to $1929.41 J$ of the EMRI, leading therefore to an increase of $13.1 \%$. This energy increase is justified by the velocity correction introduced by the EMRI external force, which is quantified by the $\varepsilon v_{T O T}$ and $\varepsilon v_{B}$, decreased by $32.4 \%$ and $28.0 \%$ respectively with the introduction of the EMRI external force. However the fact that the 4D Flow CMR velocity values near the wall were er- 

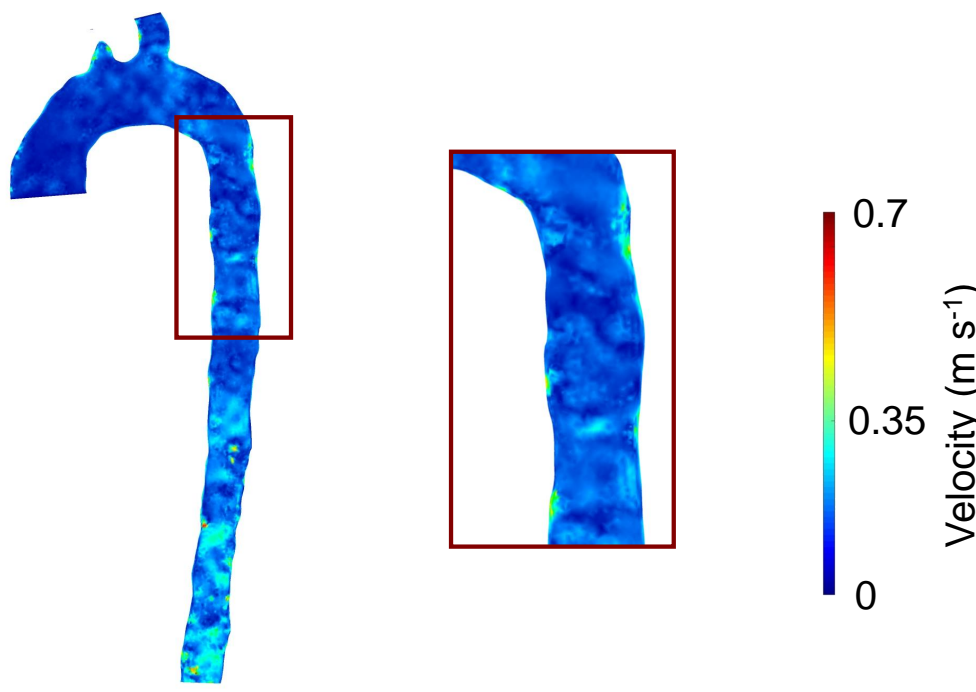

Figure 5.13: Velocity difference between EMRI and 4D Flow CMR in the sagittal section of the aorta $(\mathrm{t}=0.2348 \mathrm{~s})$ with an area magnified.

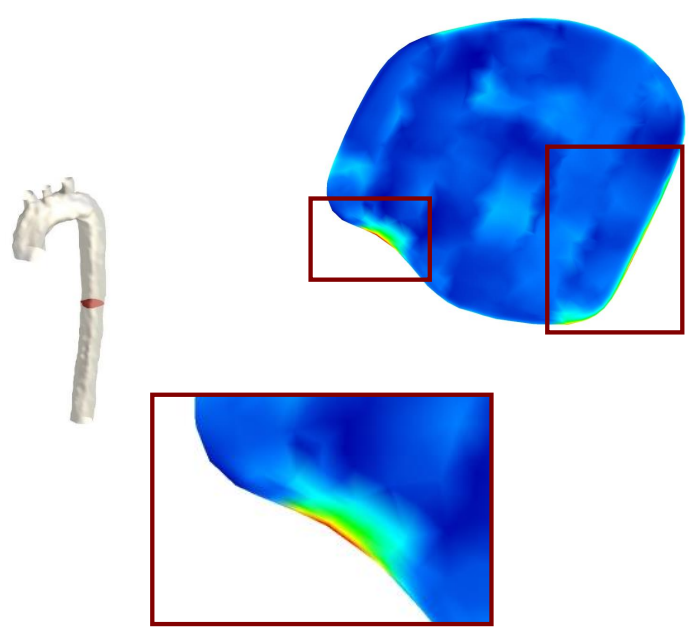

a)

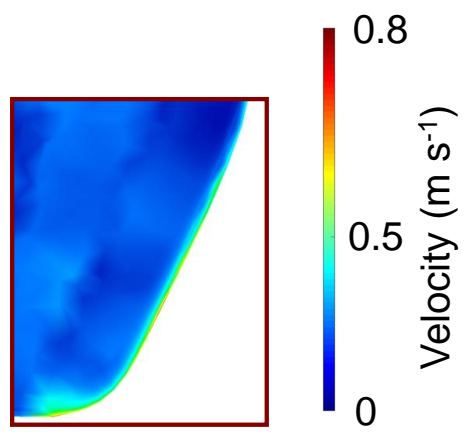

b)

Figure 5.14: Velocity difference between EMRI and 4D Flow CMR in a transverse section of the aorta $(\mathrm{t}=0.2348 \mathrm{~s})$ with two area magnified $(\mathrm{a}, \mathrm{b})$.

roneous is certainly leading to a large force term to ensure the non-slip boundary condition. This effect is reflected in the WSS distribution (Figure 5.16). The WSS computed from the processed 4D Flow MRI is in the range $0-3.5 P a$, consistent with 4D Flow CMR based studies [12], while the WSS computed with CFD and EMRI shows higher values, a trend in agreement with the literature when compar- 


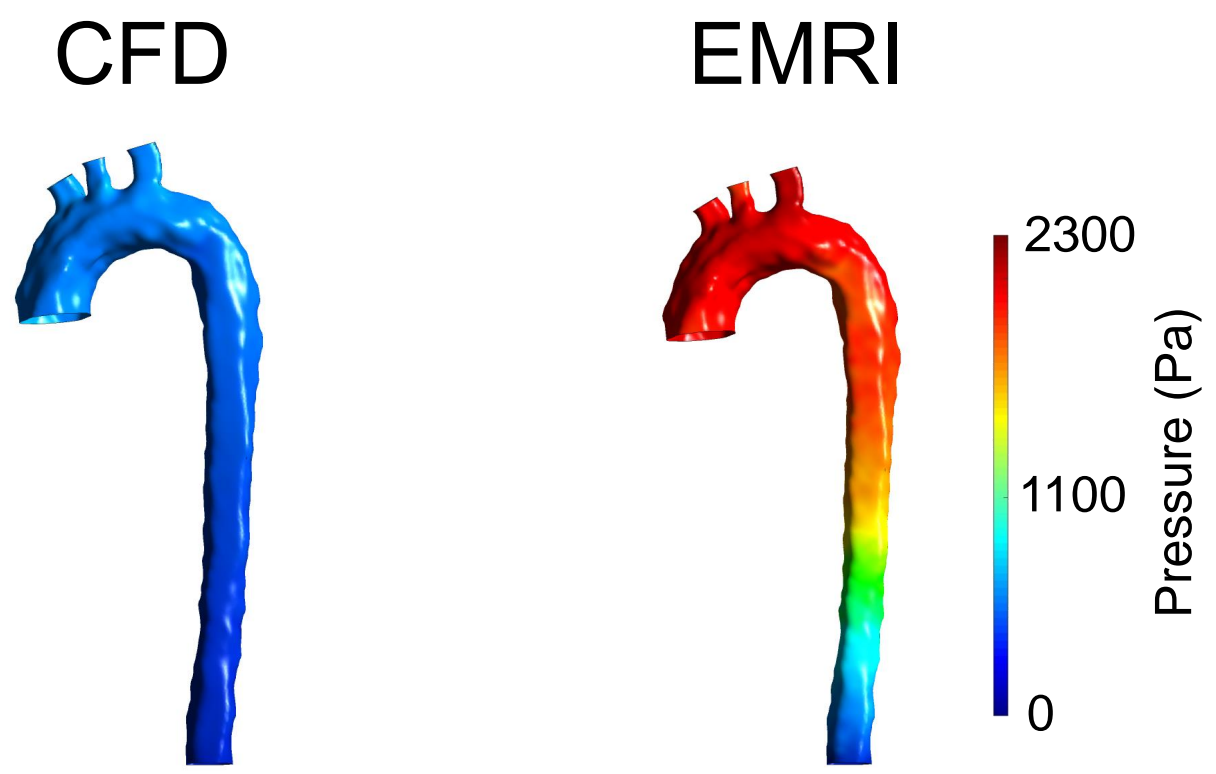

Figure 5.15: Pressure maps of the aorta $(t=0.2348 \mathrm{~s})$ on the wall with CFD and EMRI

ing MRI-derived and CFD computed WSS [12, 118].

However, there is a marked difference in the WSS distribution between CFD and EMRI. EMRI results when compared to the CFD ones, show overall higher values of WSS which exceeds the values reported in literature which in a healthy aorta are should be in the range $0-7 \mathrm{~Pa}$ [59]. EMRI presents also larger areas of localised high WSS and a less uniform distribution, especially on one side of the descending aorta.

The difference in WSS maps of CFD and EMRI is due to the discrepancy in the velocity distributions discussed earlier, since EMRI provided a higher velocity gradient near the wall, reflecting the 4D Flow CMR observations. As previously pointed out, the velocity information of the layer near the wall is coming from one voxel only, constituting a very poor representation of the highly varying velocity field characterizing this area of the vessel, and therefore could lead to an erroneous velocity enforcement.

In order to take this into account a wall function in the expression of the EMRI 
4D Flow CMR
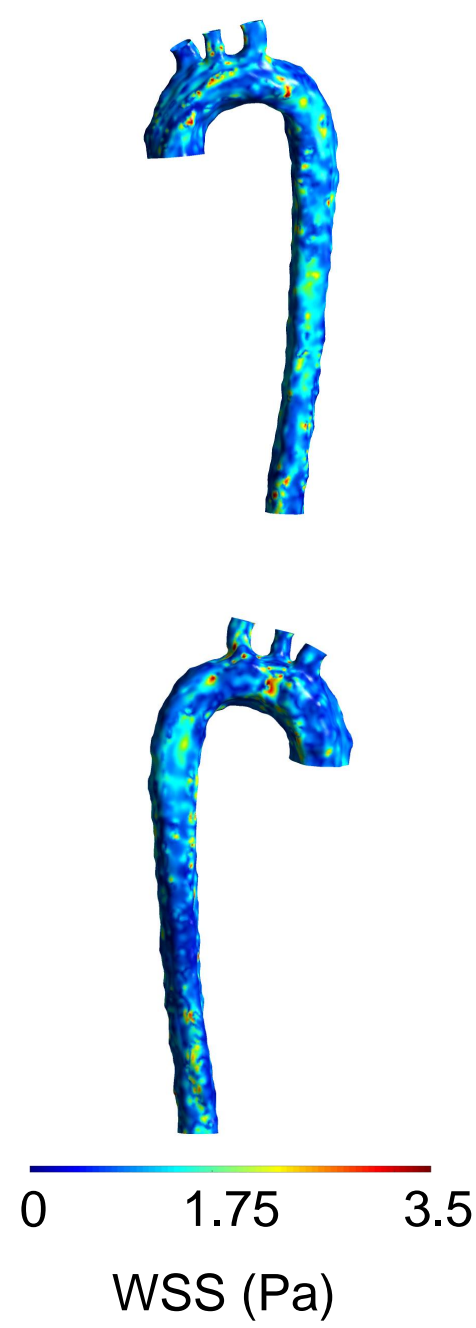

CFD EMRI
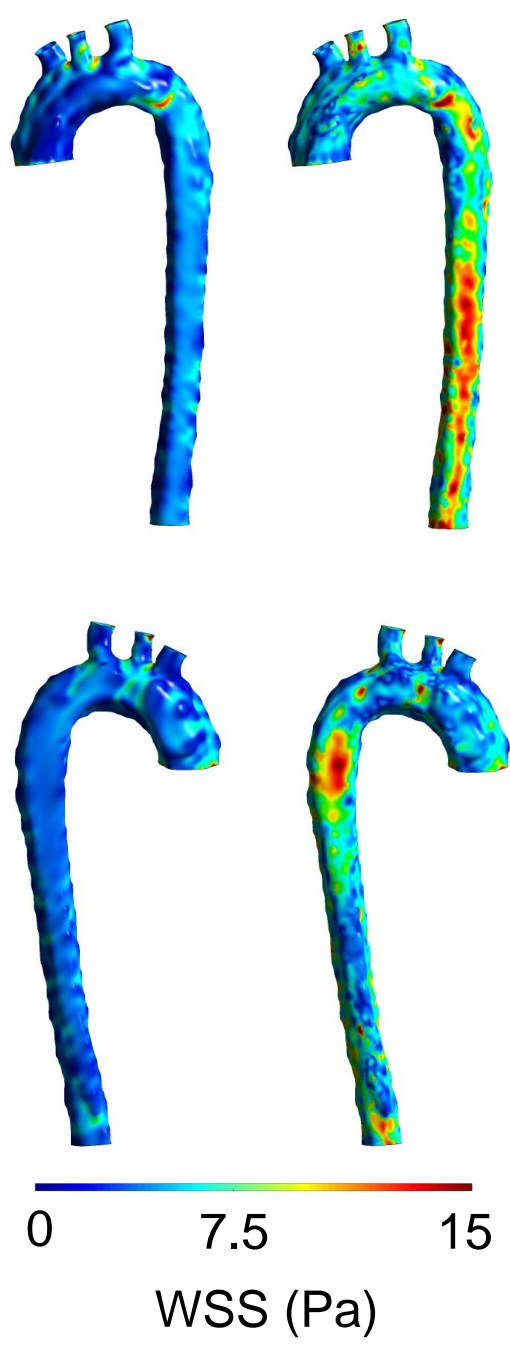

Figure 5.16: Wall shear stress maps of two sides of the aorta $(t=0.2348 \mathrm{~s})$ computed with 4D Flow CMR, CFD and EMRI.

force term was introduced (Equation 5.6) and a comparison with the standard EMRI will be carried out in the next section.

An increase in the pressure is also observed when comparing CFD and EMRI (Figure 5.15 and Table 5.3). It could be expected that the pressure obtained with EMRI would be the one associated with the 'corrected version' of the measured velocity field, however this reproducibility has been found infringed [95]. In order to understand this, the NS equations could be taken in consideration, and rewritten in a compact notation for the EMRI model (equation 5.8,5.9): 


$$
\rho\left(\frac{\partial \vec{v}_{E M R I}}{\partial t}+\left(\vec{v}_{E M R I} \cdot \nabla\right) \vec{v}_{E M R I}\right)=\mu \Delta \vec{v}_{E M R I}-\nabla p_{E M R I}+\vec{f}
$$

$$
\nabla \cdot \vec{v}_{E M R I}=0
$$

where $v_{E M R I}=\left(u_{E M R I}, v_{E M R I}, w_{E M R I}\right)$ is the velocity vector field, $p_{E M R I}$ is the pressure, $\vec{f}=\left(f_{x}, f_{y}, f_{z}\right)$ is the external force field. After computing the divergence of the Equation 5.8, and substituting Equation 5.9 in it, the following expression for the pressure is obtained (equation 5.2.1.2):

$$
\Delta p_{E M R I}=-\nabla \cdot \rho\left(\vec{v}_{E M R I} \cdot \nabla\right) \vec{v}_{E M R I}+\nabla \cdot \vec{f}
$$

If $\vec{v}_{\text {real }}$ is the velocity field satisfying the NS equations of the system studied, then equation would become:

$$
\Delta p_{\text {real }}=-\nabla \cdot \rho\left(\vec{v}_{\text {real }} \cdot \nabla\right) \vec{v}_{\text {real }}
$$

If we assume that the $v_{E M} \vec{M} \approx v_{\text {real }}$, then equation 5.2.1.2, could be rewritten as :

$$
\Delta p_{E M R I}=\Delta p_{\text {real }}+\nabla \cdot \vec{f}
$$

This pressure difference is the result of two terms: one associated with the rotational component of the force term and the other one associated with the irrotational part. The first one is the result of the velocity field imposed through the force, and the other one is the results of the non divergence-free part of the force, that deteriorates the pressure field. In order to give a correct estimate of the pressure field, a correction term needs to be introduced. This will be part of a future work. 


\subsubsection{Aortic flow at late systole: comparing standard EMRI and EMRI Wall function}

\subsubsection{Sensitivity test}

A sensitivity test to determine the appropriate value of $\triangle P i x(\mathrm{~mm})$ in the $\phi\left(d_{W A L L}\right)$ expression was carried out. The values of kinetic energy, $\varepsilon v_{T O T}$ and $\varepsilon v_{B}$ for the different values of $\Delta$ Pix $(\mathrm{mm})$ are reported in Table 5.4.

Table 5.4: Quantities used to assess the impact of the introduction of $\phi\left(d_{W A L L}\right)$ for different $\Delta$ Pix values.

\begin{tabular}{|c|c|c|c|c|}
\hline \hline$\Delta$ Pix $(\mathrm{mm})$ & $\begin{array}{c}\text { Kinetic } \\
\text { Energy }(J)\end{array}$ & $\begin{array}{c}\varepsilon v_{T O T} \\
\left(m s^{-1}\right)\end{array}$ & $\begin{array}{c}\varepsilon v_{B} \\
\left(m s^{-1}\right)\end{array}$ & Iterations \\
\hline No Force & 1702.32 & 0.197843 & 0.0691595 & 100 \\
0 & 1931.76 & 0.133665 & 0.049792 & 42 \\
0.25 & 1884.4 & 0.134628 & 0.044753 & 42 \\
0.5 & 1796.42 & 0.136494 & 0.042138 & 42 \\
0.75 & 1715.78 & 0.138614 & 0.040351 & 43 \\
1 & 1658.38 & 0.140705 & 0.038843 & 43 \\
1.25 & 1625.54 & 0.142592 & 0.038503 & 43 \\
1.5 & 1604.23 & 0.144321 & 0.038360 & 43 \\
\hline \hline
\end{tabular}

A $\triangle$ Pix of $0.75 \mathrm{~mm}$ was chosen because it minimizes $\varepsilon v_{T O T}$ as well as $\varepsilon v_{B}$. Moreover using this parameter the energy of the system is only $0.79 \%$ higher with respect to the case without external force. Higher values of $\Delta$ Pix lead to lower errors in the area near the wall, but lead overall to a larger error if the whole computational volume is considered. In Table 5.4 also the iterations number for the different cases are shown; there is little impact played by the introduction of the $\phi\left(d_{W A L L}\right)$ in the EMRI framework, while the number of iterations are reduce by $64 \%$ with respect to the case without external force.

The maps in Figure 5.17 show the spatial distribution of $\phi\left(d_{W A L L}\right)$ for $\Delta$ Pix $(\mathrm{mm})$ of $0.75 \mathrm{~mm}$ when evaluated on the sagittal cutting plane and transversal cross section of the aorta.

In Figure 5.18 the velocity profile extracted from raw 4D Flow CMR $\left(v_{M R I}\right)$, computed with the $\left(\phi\left(d_{W A L L}\right) \cdot v_{M R I}\right)$ and EMRI are shown in a line crossing the 


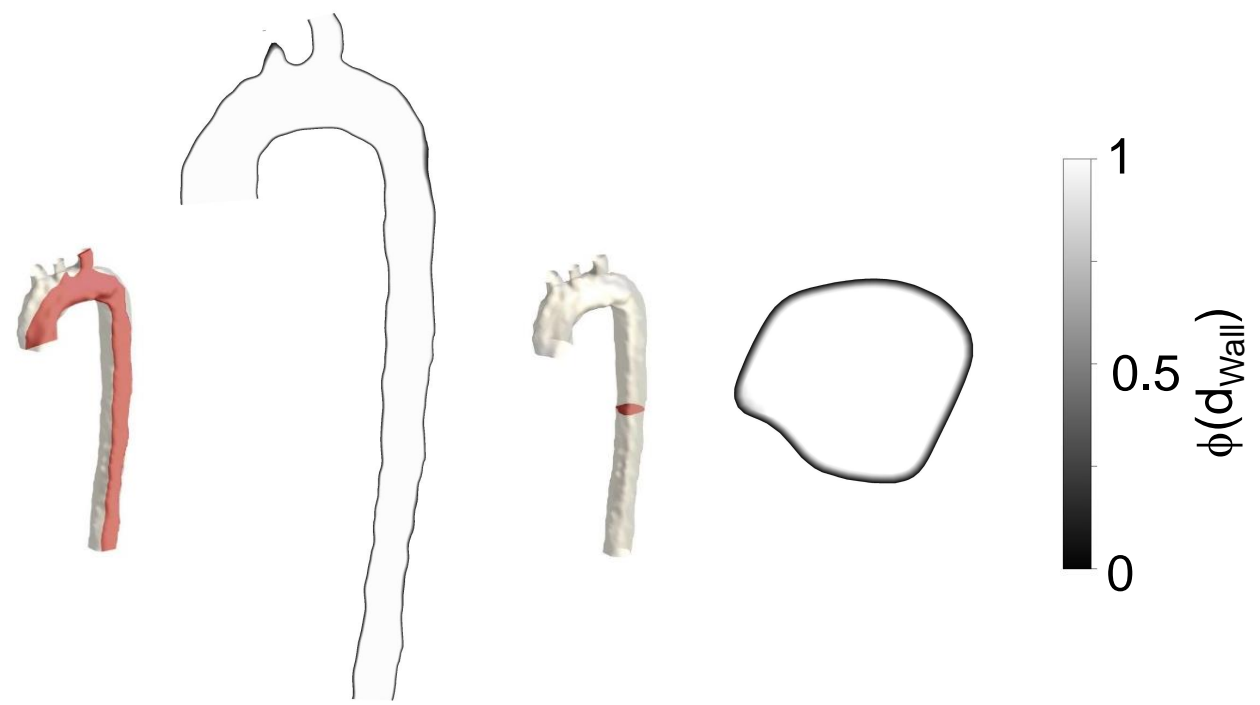

Figure 5.17: Diagram showing the trend of $\phi\left(d_{W A L L}\right)$ with $\Delta$ Pix $(\mathrm{mm})$ of $0.75 \mathrm{~mm}$ in the sagittal cutting plane (on the left) and transversal cross section (on the right) of a patient specific aorta .

descending aorta (shown also in the left side of the figure).

In Figure 5.19 the velocity surfaces computed from raw 4D Flow CMR data $\left(v_{M R I}\right)$, from the $\left(\phi\left(d_{W A L L}\right) \cdot v_{M R I}\right)$ and from EMRI data are shown for a transverse plane of the descending aorta (Figure $5.10 \mathrm{~b}$ ) at late systole.

These figures illustrate the huge impact that the EMRI has on the velocity field, and the substantial correction of the velocity field, which is not limited to the boundary region, but also the bulk flow.

\subsubsection{Blood flow velocities}

The velocity maps in the sagittal cutting plane and transversal cross section of the aorta, computed with EMRI and EMRI Wall function are shown in Figure 5.20. Overall the velocities obtained with EMRI Wall function are similar to the one obtained with EMRI. However, considering the saggital velocity maps (top row of Figure 5.20), it is noticeable how the area in proximity of the wall tends to have more smoothly-varying velocity values. This behaviour is evident by looking at the 

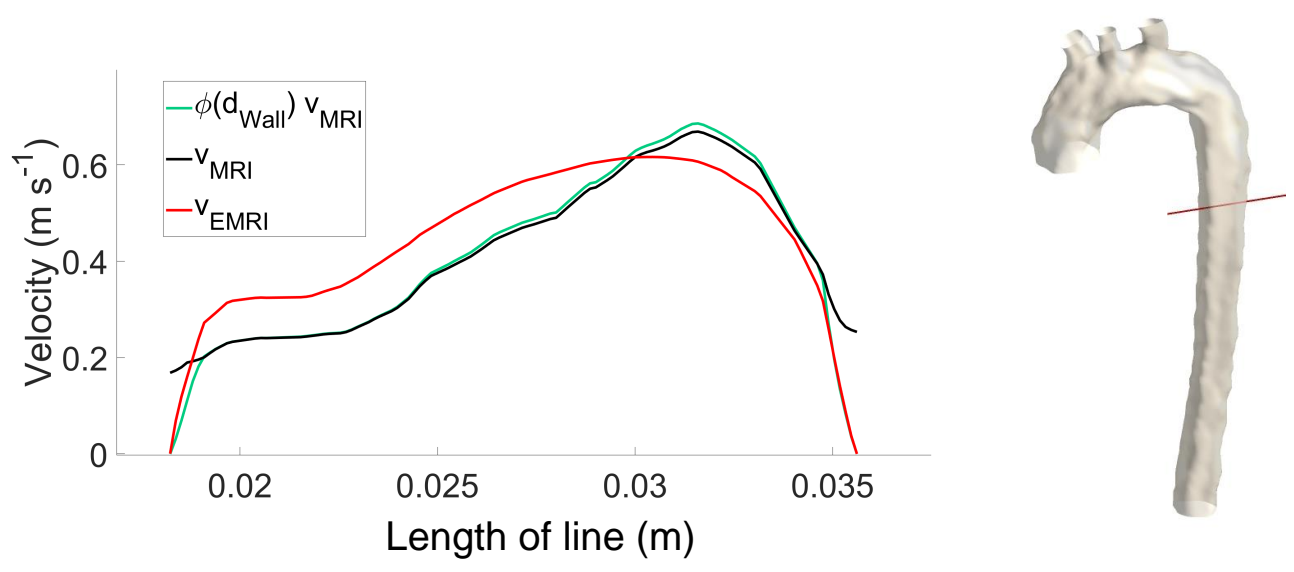

Figure 5.18: Raw 4D Flow CMR velocity profile and processed one at $t=0.2348 \mathrm{~s}$ from the beginning of the systole, computed on the line crossing the descending aorta (on the right of the picture).

$\mathrm{V}_{\mathrm{MRI}}$

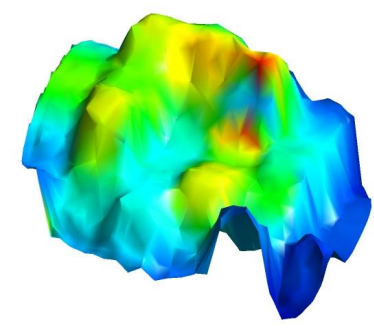

$\phi\left(\mathrm{d}_{\text {Wall }}\right) \mathrm{v}_{\mathrm{MRI}}$

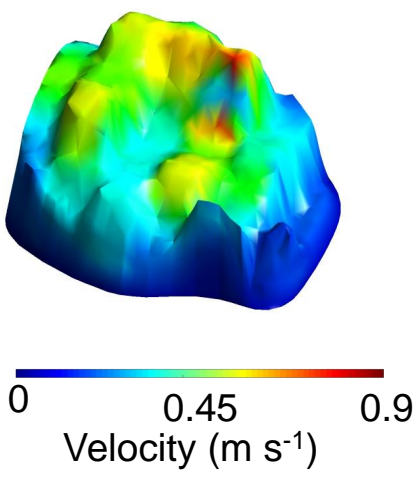

$\mathrm{V}_{\mathrm{EMRI}}$

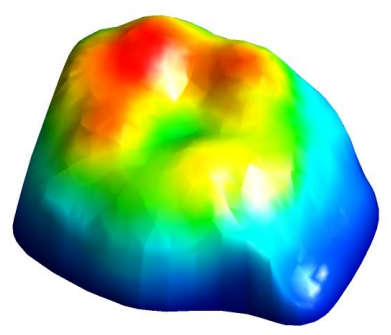

Figure 5.19: Velocity surfaces computed from raw 4D Flow CMR data $\left(v_{M R I}\right)$, from the $\left(\phi\left(d_{W A L L}\right) \cdot v_{M R I}\right)$ and from EMRI data at $\mathrm{t}=0.2348 \mathrm{~s}$ from the beginning of the systole, in a transverse plane of the descending aorta (shown Figure 5.10 b).

transversal cross sections (bottom row of Figure 5.20). To this purpose a portion of the latter was magnified (Figure 5.21).

It is clear that the function introduced in the EMRI Wall function allows to smooth the $v_{M R I}$ in the wall boundary region, even more than EMRI alone, in par- 
ticular when the $v_{M R I} \neq 0$, reducing the impact on the energy of the system as discussed previously. This is illustrated by Figure 5.22 where the velocity difference $\left(\mathrm{v}-\mathrm{v}_{M R I}\right)$ maps of the aorta computed with EMRI and EMRI Wall function in the sagittal cutting plane (first row) and transversal cross section (second row) are shown. The residual error on the area in proximity of the wall dramatically decreases with the introduction of the wall function in the EMRI model.

The effect of the function $\phi\left(d_{W A L L}\right)$ is reflected also in the WSS maps; in Figure 5.23 the WSS maps of two sides of the aorta computed with EMRI and EMRI Wall function are compared. This analysis shows how EMRI Wall function leads to lower values of WSS, smoothing the high WSS peaks obtained by the standard EMRI described previously.

\subsubsection{Aortic flow studied with EMRI wall function and time re- solved CFD}

Blood flow was assessed using 4D Flow CMR, EMRI (wall function) and time resolved CFD in four phases of the aortic cycle (blue dots in Figure 5.4): accelerating phase (instant $1, t=0.0470 \mathrm{~s}$ from the start of the systole), peak systole (instant 2, $t=0.0939 \mathrm{~s}$ from the start of the systole), decelerating phase (instant $3, t=0.2348 \mathrm{~s}$ from the start of the systole) and mid diastole (instant $4, t=0.4695 \mathrm{~s}$ from the start of the systole). Overall the velocity magnitude across the cycle is consistent with the velocity values of an healthy aorta, which are in the range $0-1.4 \mathrm{~ms}^{-1}$. The CFD and the EMRI show smoother velocity maps (e.g. Figure 5.36) with respect to the 4D Flow CMR ones, which shows a noisy behaviour, highlighted also by the vector plots (e.g. Figure 5.38). The four phases are discussed individually in the next subsections.

\subsubsection{Acceleration phase}

During early systole, the flow field in the aorta starts developing, with an increase of the axial velocity, with a profile skewed towards the inner wall, which is characterized by higher velocities [50]. This trend characterizes the CFD and more markedly EMRI velocity maps (Figure 5.24). It is however not clearly visible in the 4D Flow 
CMR velocity map.

The velocity vectors maps (Figures 5.25 and 5.26) show more clearly the flow paths. In the ascending aorta the CFD is characterized by a more symmetric velocity profile across the vessel width, with respect to the EMRI, that shows the higher velocities in the inner part of the aorta.

In the aortic arch the CFD computed flow presents the same trend of the ascending aorta, showing a symmetric velocity distribution, while the EMRI velocity field is skewed towards the outer wall of the arch, presenting lower velocities in the inner part.

The descending aorta is the segment characterized by the higher velocities, with a peak of $0.8-0.9 \mathrm{~ms}^{-1}$ in the EMRI, while the CFD shows slightly lower velocities. On the contrary the 4D Flow CMR velocity map shows very noisy patterns, which could be justified by the temporal averaging and the low temporal resolution, which might deteriorate the flow field in this cardiac phase of highly accelerated flow. This observation is also confirmed by the velocity vectors map. On the other hand, in this segment of the aorta the CFD and the EMRI vector plots of the in-plane velocity are characterized by a similar trend, with cross sectional velocities directed towards the inner part of the vessel (Figures 5.28 and 5.29). On the opposite the velocity magnitudes show again the same trend with higher values in the EMRI model (Figures 5.27).

\subsubsection{Systolic peak}

During the systole, the region of higher velocity is migrated away from the inner wall towards the axis of the aorta (Figure 5.30). This is more visible in the EMRI and in the 4D Flow CMR (even though the velocity map appears noisy). The sagittal section vector plots (Figures 5.31 and 5.32) show that the velocity is still symmetric across the vessel width. However the cross sectional vector plots (Figure 5.35), particularly for CFD and EMRI, show the presence of right handed helical flow (clockwise when viewed in the direction of forward movement of the flow) at the left and the right hand side (with respect to the symmetry plane of the arch) of the descendent aorta, right after the distal segment of the aortic arch [27, 12]. 
The descending aorta is characterized by the higher velocity of the cardiac cycle (Figure 5.30). In particular the EMRI velocity map shows the higher velocity magnitude, peaking to $1.4 \mathrm{~ms}^{-1}$, contrarily to the CFD and 4D Flow CMR that show much smaller velocity values. The velocity distribution in the considered cross section of descending aorta (Figure 5.33) shows also the same trend, with pronounced differences in the magnitude; the EMRI shows the same trend with respect to 4D Flow CMR, while CFD has a more homogeneous distribution of the velocities. In the vector plots of the same section (Figure 5.34) the EMRI and the CFD exhibit a similar secondary flow, while the MRI is characterized by more complex flow structure.

\subsubsection{Late systole}

In late systole more accentuated flow separation occurs and the highest velocity stream migrates towards the outer wall of the distal aortic arch. This behaviour is correctly depicted by the EMRI, and partially by the 4D Flow CMR, that show distinctly higher velocities in the outer wall in the distal aortic arch and the outer wall of the first portion of the descending aorta (Figure 5.36). The CFD shows also in this case a more symmetric distribution of the velocities across the vessel.

In this cardiac phase secondary helical flows develop; in particular flow separates from the inner wall of the distal arch, and oblique streamlines appear, causing right handed helical pattern of flow that curl towards the anterior part of the descending aorta [27]. These flow features are visible in Figures 5.37 and 5.38 by the vector distributions of EMRI and 4D Flow CMR in the descending aorta.

A more complete view on the secondary flow development is given by the cross sectional flow (Figures 5.40 and 5.41). As the sections in the first row of Figure 5.41 indicates, all the three modalities show the presence of secondary flow, located however in a different position in the CFD with respect to the EMRI and 4D Flow CMR.

The same observation applies to the lower section of the descending aorta (Figure 5.40), which is characterized also by a different distribution of the velocity magnitude (Figure 5.39). In particular the EMRI model reproduces the experimen- 
tal velocity, showing a less symmetric velocity distribution with respect to the CFD model.

\subsubsection{Mid diastole}

Diastole is characterized by a stronger reduction of velocity, as showed in the velocity magnitude maps (Figure 5.42), and in particular of the axial flow component (Figures 5.43 and 5.44). In this phase of the cardiac cycle, the 4D Flow CMR suffers of errors due to low sensitivity of the velocity encoding gradients to small velocities, as the velocity range is much lower than the $v_{E N C}$. As a consequence the EMRI velocity distribution results less homogeneous than the CFD one (Figures 5.42 and 5.45).

In this phase secondary helical and retrograde flows persists (Figures 5.46 and 5.47). Helical flow patterns are very subject specific and they are dependent on the geometry of the aorta [27], therefore assessing the performance of the suggested methodology with respect to CFD or 4D Flow CMR is challenging.

The ability of EMRI to estimate more complex velocity patterns than CFD offers the potential advantage of reproducing flow/vortex structures closer to those observed in vivo in the aorta [33]. Overall the vortex structure computed with EMRI is more heterogeneous compared to the standard CFD and, although CFD and EMRI share the main flow structures, the helical structures and the swirling paths show a very different behaviour [95].

\subsubsection{WSS}

In the Figures 5.48 - 5.51 the WSS computed with CFD, EMRI (Wall function) and computed with 4D Flow CMR are shown for the 4 selected instants of the cardiac cycle. The MRI computed WSS shows results in agreement with MRI studies with values in the range $0-3 P a[12,119,120,29,9]$. The estimation of WSS is still a challenging task and there is no standardized methodology [121]. Usually more complex fitting of 4D Flow CMR measurements are performed such as spline fitting [121] or data denoising to improve the velocity estimation and therefore have a more accurate estimation of the WSS [115]. 
In general, WSS calculated using computational models shows higher values of WSS with respect to the MRI-derived one, which is mainly due to the limited spatial resolution of MRI measurements $[63,12]$. This is the case in the presented study, where both the computational models show high WSS values which range between $0-15 \mathrm{~Pa}$. This behaviour could be consequence of a segmentation error, originating from the low spatial resolution of the data $1.36 \times 1.36 \times 3 \mathrm{~mm}^{3}$, as it is not characterizing the EMRI model only, but also the CFD one. Moreover the error in the segmentation would particularly affect EMRI as by reducing the vessel lumen, the velocity field informing the simulation would be characterized by higher values of velocity in the regions in proximity of the wall and therefore cause higher velocity gradients, and thus high values of WSS.

Overall the spatial distribution of the WSS shows consistency with the literature $[115,12]$. In particular the WSS maps show an increasing trend when moving from the anterior wall of the aortic arch and moving along the descending aorta. Moreover the high WSS region increase in size when moving from the arch down to the descending aorta $[115,9]$. This is clear in the computational models maps.

Table 5.5 shows the WSS computed from processed 4D Flow CMR data, time resolved CFD and EMRI, averaged over the aortic wall for the considered instants of the cardiac cycle.

Table 5.5: WSS computed from processed 4D Flow CMR data, time resolved CFD and EMRI, averaged over the aortic wall for the considered instants of the cardiac cycle.

\begin{tabular}{|c|c|c|c|}
\hline \hline $\begin{array}{c}\text { Instants } \\
(\mathrm{mm})\end{array}$ & $\begin{array}{c}W S S_{M R I} \\
(\mathrm{~Pa})\end{array}$ & $\begin{array}{c}W S S_{C F D} \\
(P a)\end{array}$ & $\begin{array}{c}W S S_{E M R I} \\
(P a)\end{array}$ \\
\hline 1 & 0.69 & 4.25 & 7.63 \\
2 & 0.84 & 4.74 & 9.27 \\
3 & 0.79 & 1.67 & 2.98 \\
4 & 0.51 & 0.37 & 1.17 \\
\hline \hline
\end{tabular}

Moreover to better summarize the WSS temporal evolution, the WSS data of each modality was normalized with respect to the time average WSS (Equation 5.13): 


$$
W S S_{t}=\frac{W S S_{t}}{\frac{1}{N} \sum_{t}^{N} W S S_{t}}
$$

where $t$ is the time instant and $N$ is the number of samples considered (in this case 4).

The temporal evolution of the WSS shows a qualitative agreement with published studies $[115,9]$, with higher values in systole with respect to the diastole.

\subsubsection{Short segment of the descending aorta}

As demonstrated in the 2D channel results in the previous chapter, EMRI has the ability to 'accelerate' the flow development along an enclosed conduit. This suggests that this method could allow an accurate computation of the flow even for a short region of interest.

In order to verify this potential advantage, a series of computations for a short segment of the descending aorta were carried out using EMRI and CFD. Resulting velocity maps are shown in Figure 5.53.

The comparison of the velocity maps in the longitudinal plane shows that the velocity profile derived from standard CFD is more skewed towards the wall on the right side. Here, the velocity distribution at the inlet (top end of the domain), which is already skewed to the right, was prescribed as the inflow boundary condition, and the velocity patterns in the downstream in CFD appears to be the continuation of the inflow profile.

On the other hand, the EMRI velocity is more centred, with a peak velocity closer the axis of the aorta. This velocity profile closely matches that obtained with the EMRI analysis of the entire aorta, confirming also this expected advantage of the proposed approach. The EMRI approach enables to compensate for errors in the boundary conditions and to alleviate the dependency of the CFD model from the modelling assumptions by embedding the in-vivo flow complexity via the experimental velocity enforcement.

A similar trend can be seen in the cross-sectional plane; the velocity is more skewed in CFD towards the left side of the aorta, whereas the EMRI-derived veloc- 
ity profile is more evenly distributed, closely correlated to the velocity pattern in the entire aorta EMRI.

\subsection{Final remarks and limitations}

This feasibility study showed how EMRI was able to correctly reproduce the complex flow patterns observed in vivo, while correcting for the experimental noise and inaccuracies, which might be detrimental especially when the resolution is limited and accelerated sequences (SENSE) are used, as in this case. The velocity magnitude and the flow paths showed a good agreement with the published works about aortic haemodynamics.

Nevertheless, the aortic flow in the in-silico models was computed under some approximations. Blood was modelled as Newtonian and the flow was assumed to be laminar. Although there is no gold standard, Newtonian as well as laminar flow approximations are extensively used in the literature $[41,53,10,62,11]$. Laminar assumption is supported by the fact that this case study is characterized by a $R e_{\text {peak }}$ lower than the $R e_{\text {crit }}$. Stalder et al. [122] used flow sensitive MRI to assess blood flow velocities and the onset of turbulence on 30 healthy subjects. In particular their findings suggest that at rest there is presence of flow instabilities but the flow is not fully turbulent. However if in healthy subject the haemodynamics is close or at the borderline of turbulence, turbulence effect might be expected for higher cardiac outputs or pulsatility, or in presence of disease [123].

The modelling of blood rheology is another complex subject, as human blood is a non-Newtonian fluid in which its viscosity depends on the shear rate. However in CFD models of large arteries it is considered a Newtonian fluid, as the shear rate is predominantly high. In fact differences between Newtonian and Non-Newtonian models characterize low flow cardiac phases. For example Caballero et al. [124] analysed three different non-Newtonian models assessing the WSS and showing that the non-Newtonian fluid features are only significant during periods where the blood flow is quite slow, and that the Newtonian model is considered as a good approximation at mid and high-flow velocities. 
A similar outcome was documented by Liu et al. [125] where Newtonian and non-Newtonian blood model was assessed in terms of distributions of luminal surface LDL concentration and oxygen flux along the wall of the human aorta. It was shown that the non-Newtonian nature of blood has little effect on the mass transport in most regions of the aorta except for the areas with flow disturbance.

As the velocity input from 4D Flow CMR to the EMRI framework is the actual in-vivo velocity field that reflects real blood rheology and flow regime, the assumptions could partially be supplemented through the EMRI analysis framework, even though an actual reference flow was not provided.

The aortic wall was assumed to be rigid. This is another approximation which is widely adopted in the literature [35, 33, 41, 11]. Lantz et al. [126] compared fluid structure interaction (FSI) with rigid walls CFD models of the aorta, showing that there is overall a good qualitative agreement on the flow patterns. Differences arise in the velocity magnitude, that is overestimated by rigid model with respect to the FSI, especially during systole due to the different vessel cross section, and area of recirculation.

In this study also WSS differences between the models were investigated. In particular instantaneous WSS pattern on the whole aorta showed big differences. In particular the larger differences are reported during systole as a consequence of the infinite wave speed of the rigid model compared to the FSI one and the diameter expansion. The same trend was reported by Reymond et al. [119] that investigated the difference between compliant and rigid walls model in a patient-specific aorta with variable thickness arterial wall.

However, even though FSI models when compared to rigid walls ones, provide a more accurate and physiologic description of the hemodynamics, this comes at the cost of highly complex numerical algorithms, increased computational costs, and the challenge of modelling patient-specific wall properties, difficult to obtain experimentally and in most of the cases unknown [127].

The presented study constitutes the first patient specific application of the EMRI, therefore the rigid wall assumption was adopted. However, as the 4D Flow 
CMR data are acquired for vessels with cyclic movement supported by the surrounding organs, more work will need to be done in the future to account for the aortic wall compliance/motion.

Additionally, clinically-used 4D Flow CMR in general has relatively low resolution that affects the accuracy of anatomical model reconstruction, which has an impact on the flow near the wall and WSS computations. However, this is inevitable in MRI-based anatomical model reconstruction and is not specific to the EMRI algorithm.

The same limitations apply for the CFD model. Moreover in this case the inlet boundary condition imposed was a flat velocity profile with the MRI derived pulsatile waveform. This assumption is based on the fact that many in-vivo studies based on hot-film anemometry on various animal models demonstrated that the velocity profile distal to the aortic valve are flat with a weak helical component $[128,129]$. However studies carried on healthy subjects using 4D Flow CMR demonstrated the presence of helical flow patterns in the aorta, resulting from the curvature of the aortic arch, the pulsatility of the flow and the torsion of the left ventricle during the heart cycle $[27,130]$. Therefore the flat velocity profile constitutes a simplification. 


\section{EMRI}

\section{EMRI \\ Wall function}
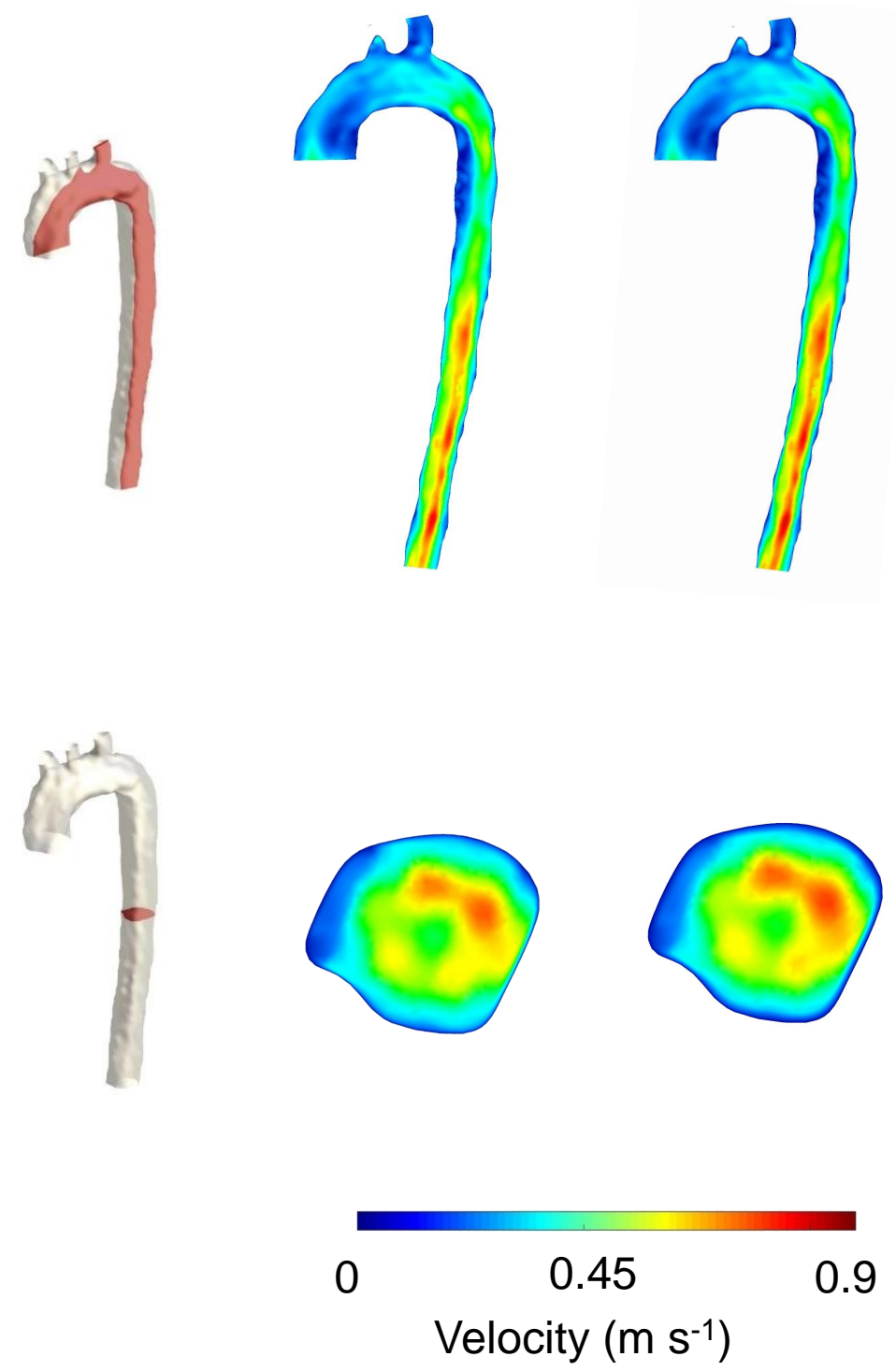

Figure 5.20: Velocity maps of the aorta $(t=0.2348 \mathrm{~s})$ computed with EMRI and EMRI with Wall function. Sagittal cutting plane (top row) and transversal cross section (bottom row). The locations of cutting planes are shown on the left panel. 
EMRI
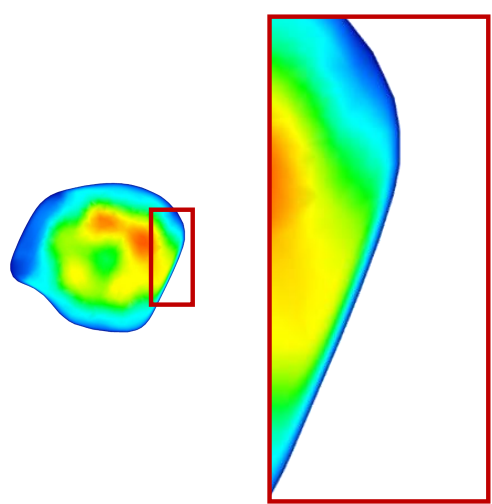

\section{EMRI} Wall function

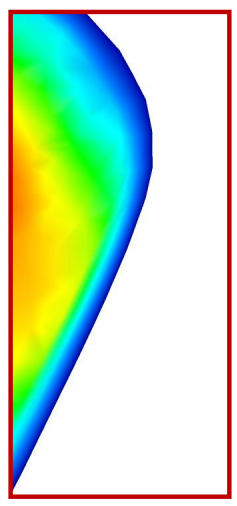

0.9

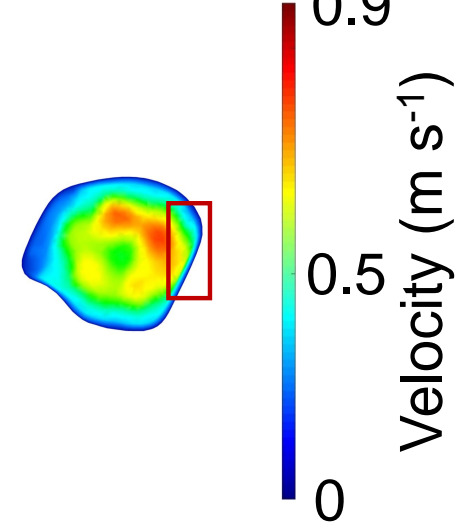

Figure 5.21: Velocity maps of the aorta $(t=0.2348 \mathrm{~s})$ computed with EMRI and EMRI Wall function in the transversal cross section (see Figure 5.20) with a magnification of an area near the wall. 


\section{EMRI}

\section{EMRI \\ Wall function}
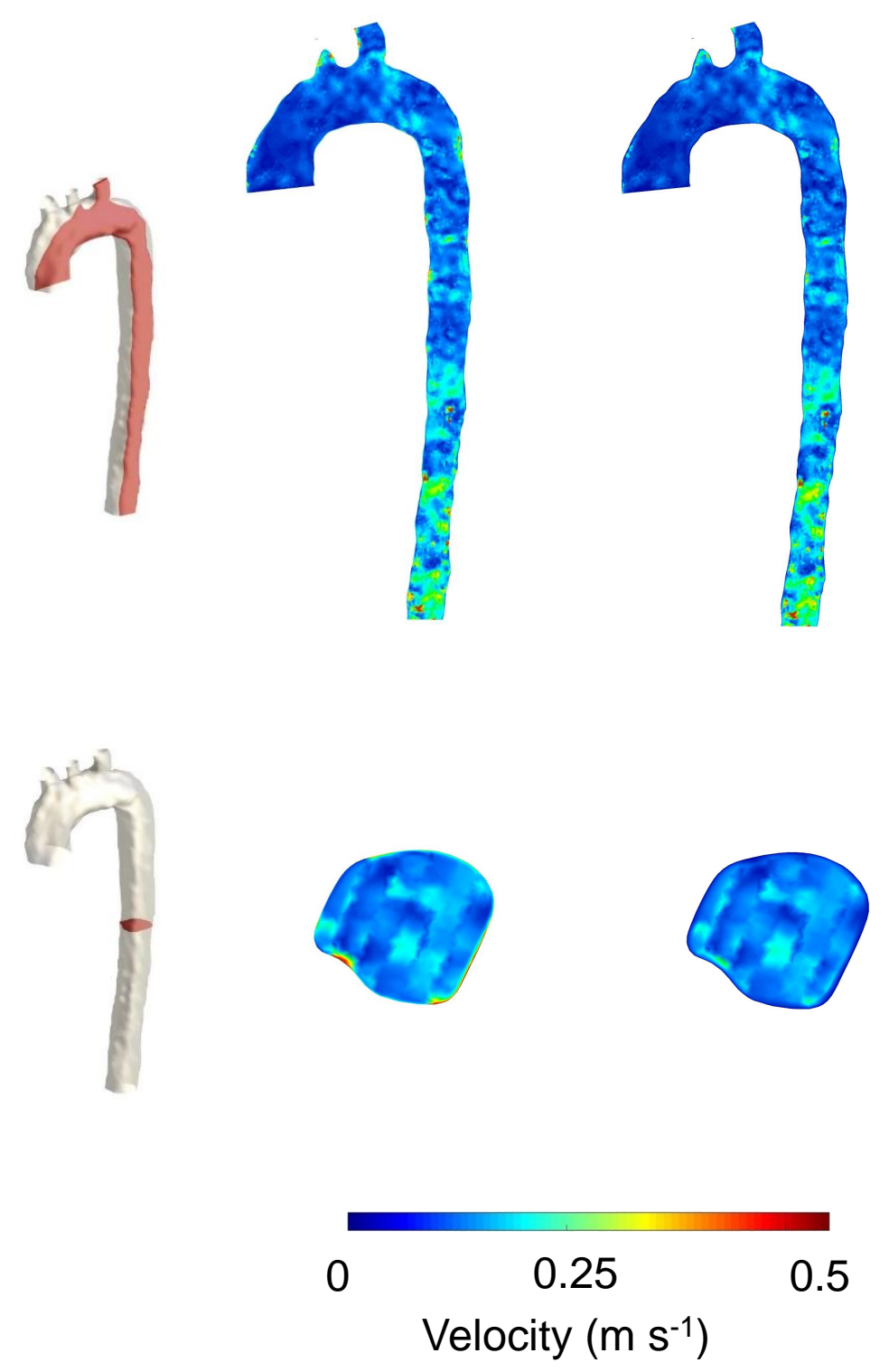

Figure 5.22: Velocity difference $\left(\mathrm{v}-\mathrm{v}_{M R I}\right)$ maps of the aorta $(\mathrm{t}=0.2348 \mathrm{~s})$ computed with EMRI and EMRI Wall function in the sagittal cutting plane (first row) and transversal cross section (second row) (the sections are showed on the left side of the picture). 
EMRI

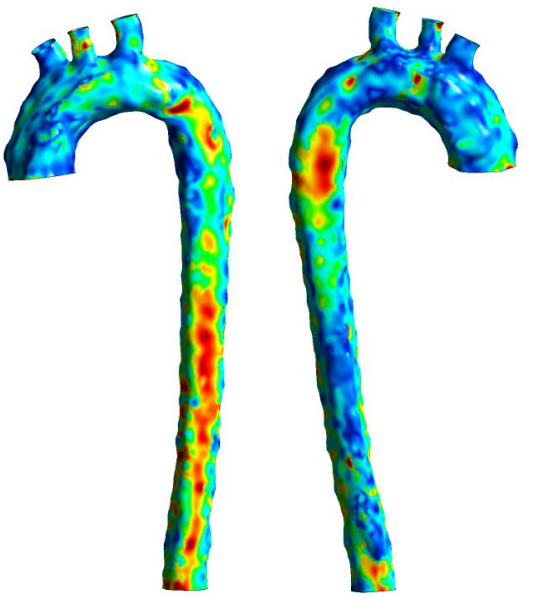

EMRI

Wall function

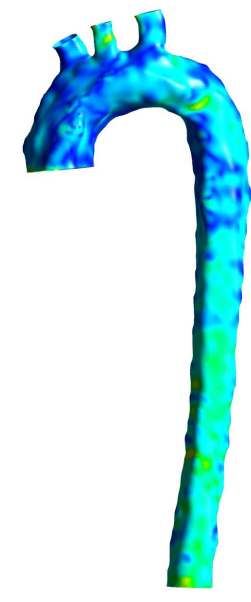

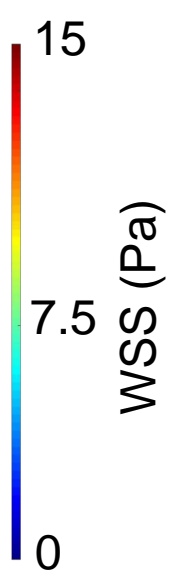

Figure 5.23: Wall shear stress maps of two sides of the aorta $(t=0.2348 \mathrm{~s})$ computed with EMRI and EMRI Wall function. 

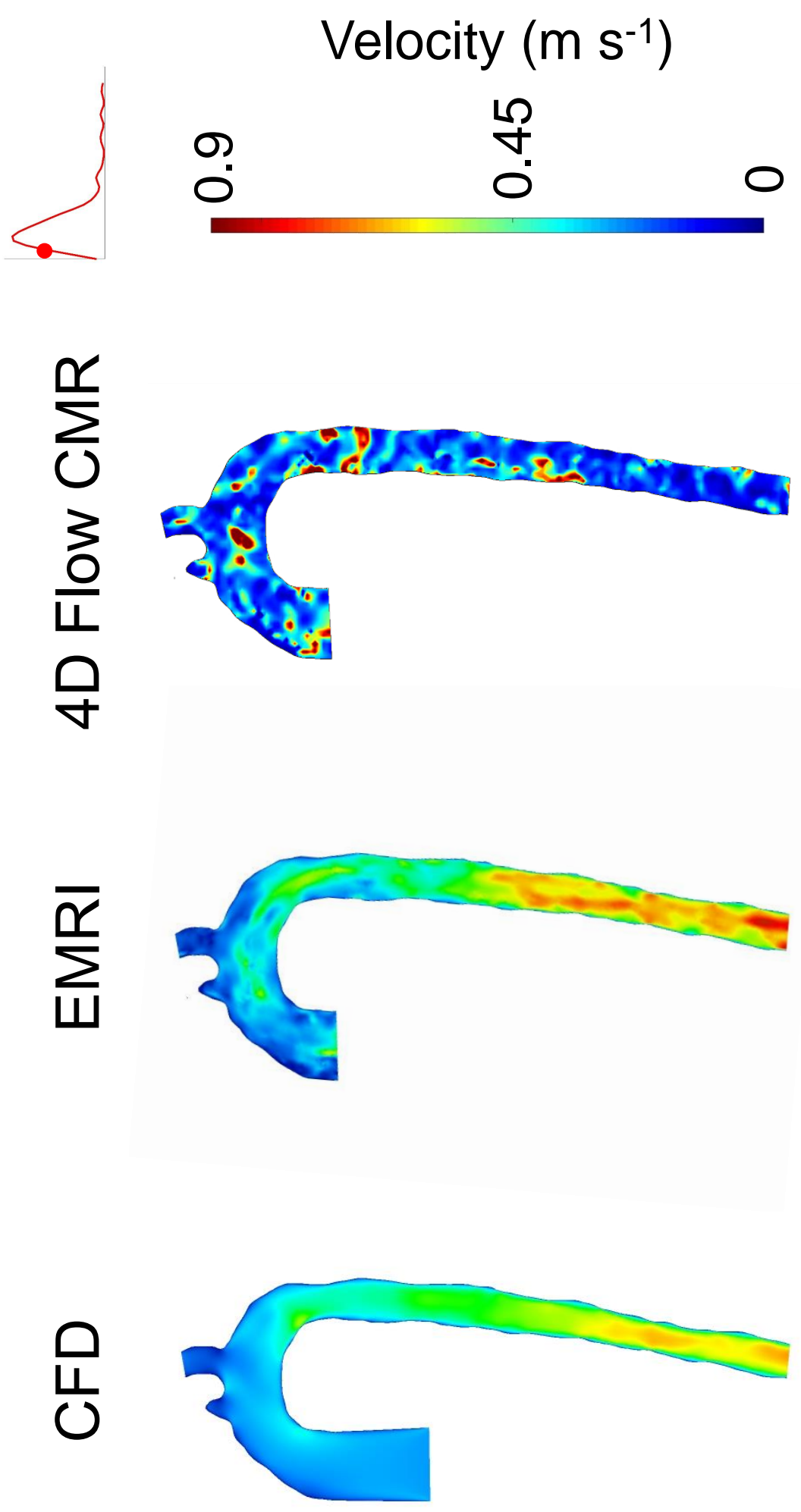

Figure 5.24: Velocity maps of the aorta at early systole (point 1) computed with CFD, EMRI (Wall function) and measured with 4D Flow CMR in the sagittal cutting plane of the aorta (Figure $5.10 \mathrm{c}$ ). 

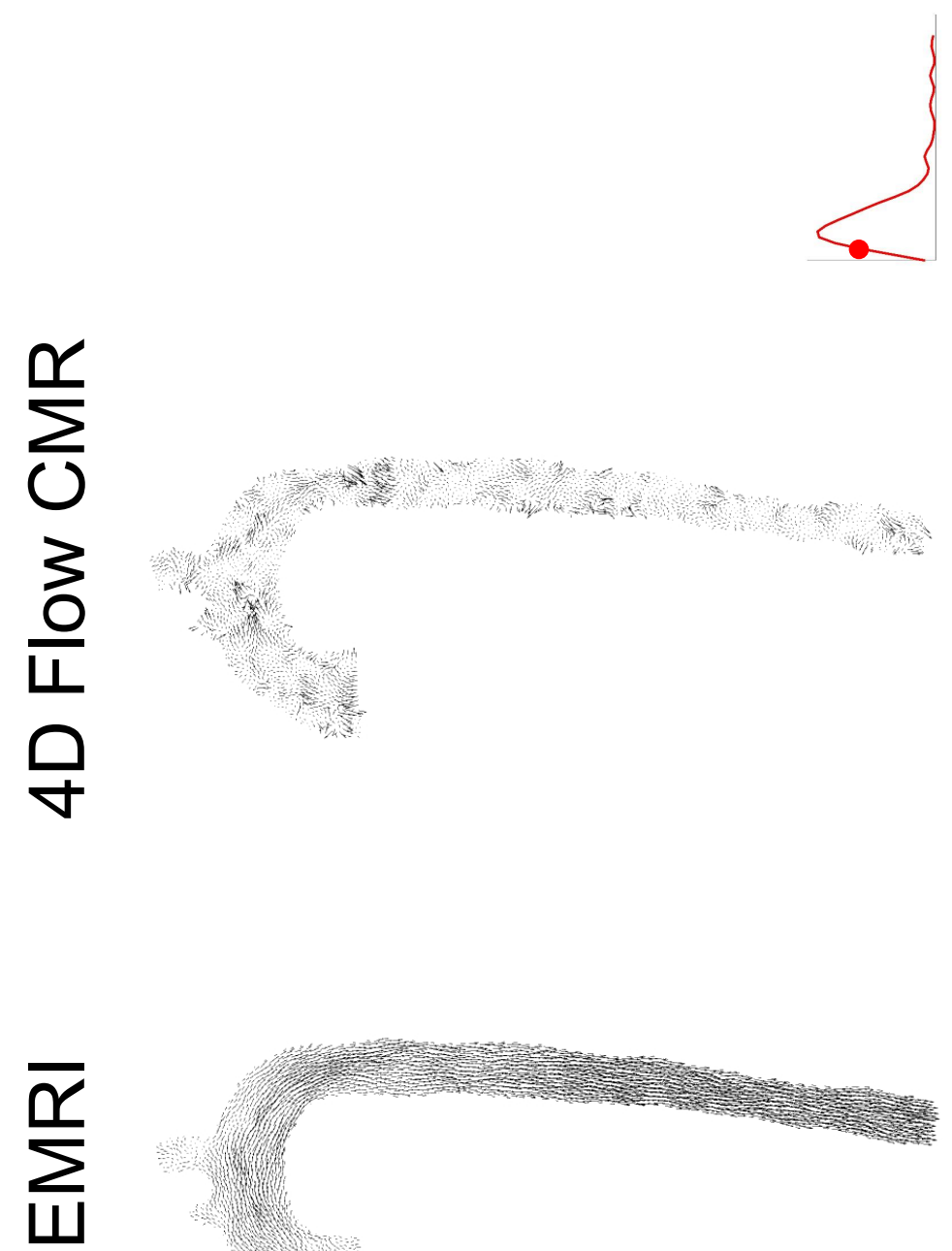

ш

닌

Figure 5.25: In plane velocity vector maps of the aorta at early systole (point 1) computed with CFD, EMRI (Wall function) and measured with 4D Flow CMR in the sagittal cutting plane of the aorta (Figure $5.10 \mathrm{c}$ ). 

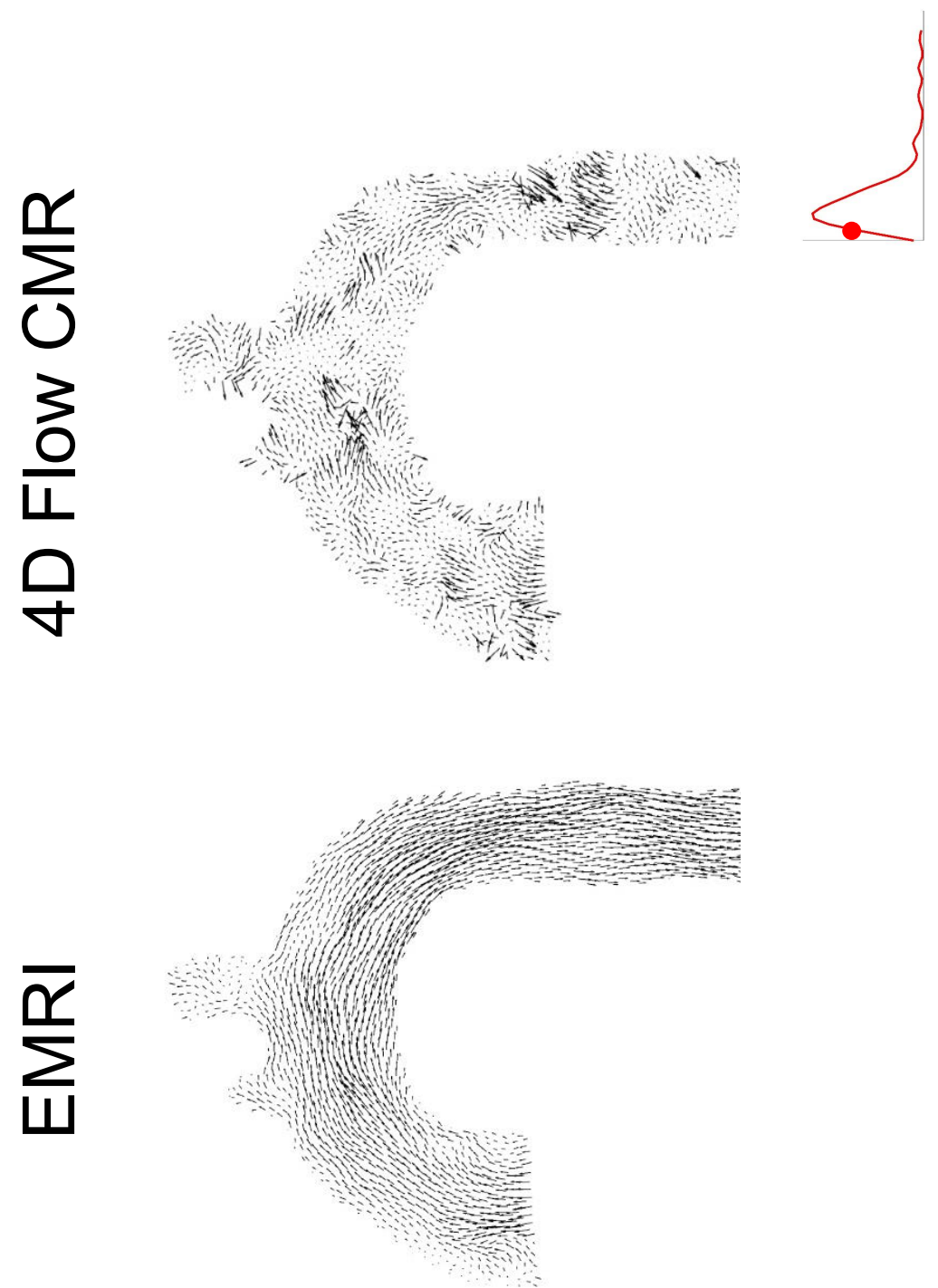

$\frac{0}{0}$

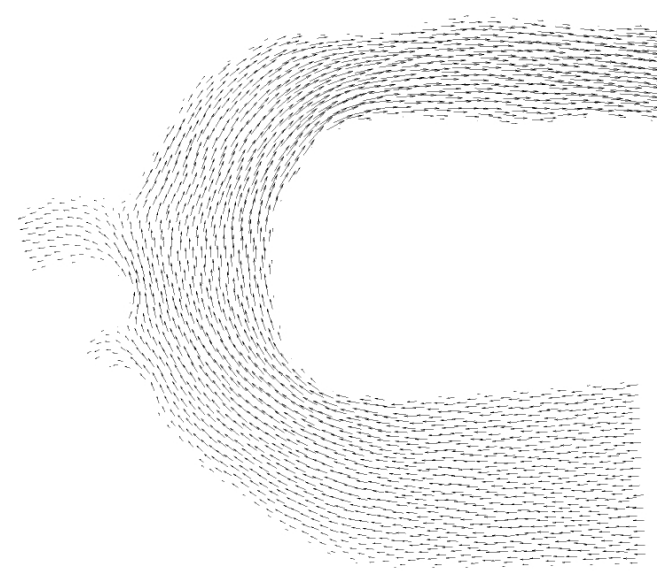

Figure 5.26: In plane velocity vector maps of the aortic arch at early systole (point 1) computed with CFD, EMRI (Wall function) and measured with 4D Flow CMR in the sagittal cutting plane of the aorta (Figure $5.10 \mathrm{c}$ ). 
CFD

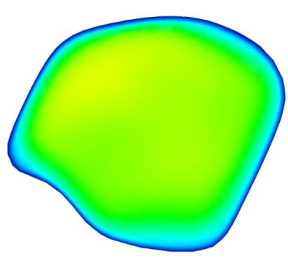

EMRI

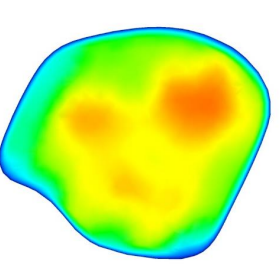

4D Flow CMR

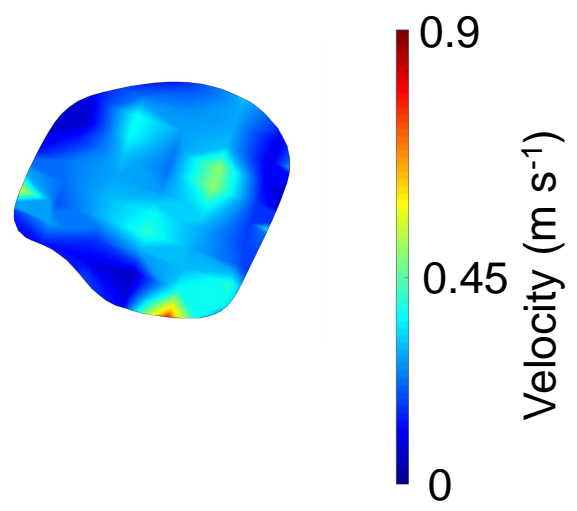

Figure 5.27: Velocity maps of the aorta at early systole (point 1) computed with CFD, EMRI (Wall function) and measured with 4D Flow CMR in the transversal cross section of the aorta (Figure $5.10 \mathrm{~b}$ ).

CFD

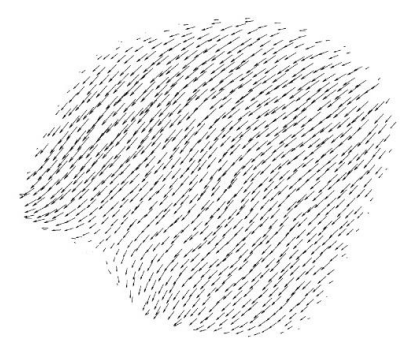

EMRI

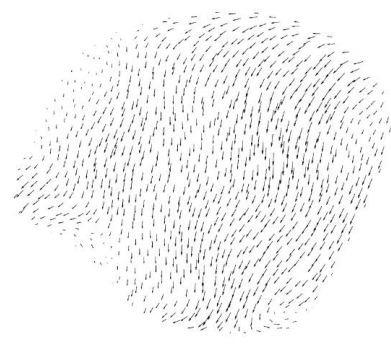

4D Flow CMR

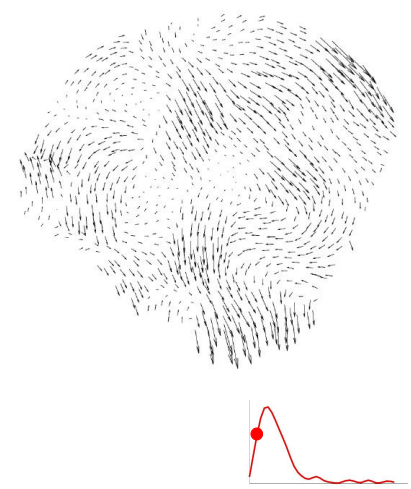

Figure 5.28: In plane velocity vector maps of the aorta at early systole (point 1) computed with CFD, EMRI (Wall function) and measured with 4D Flow CMR in the transversal cross section of the aorta (Figure $5.10 \mathrm{~b}$ ). 

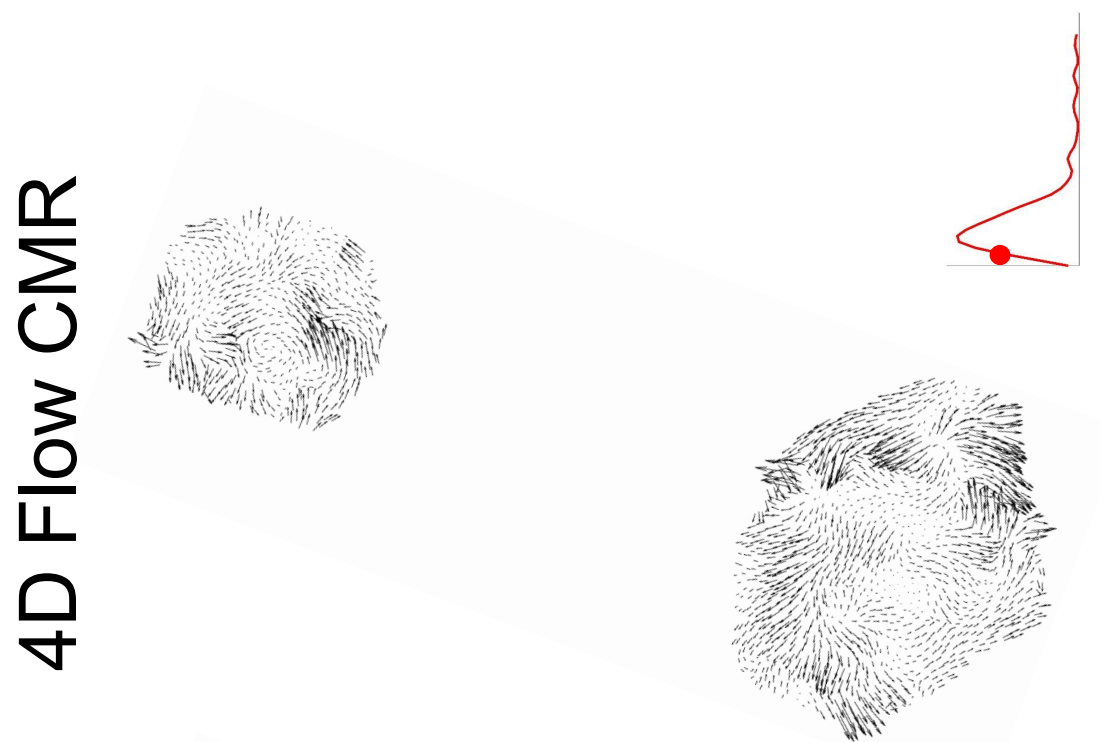

$\sum_{\amalg}^{\bar{\Upsilon}}$
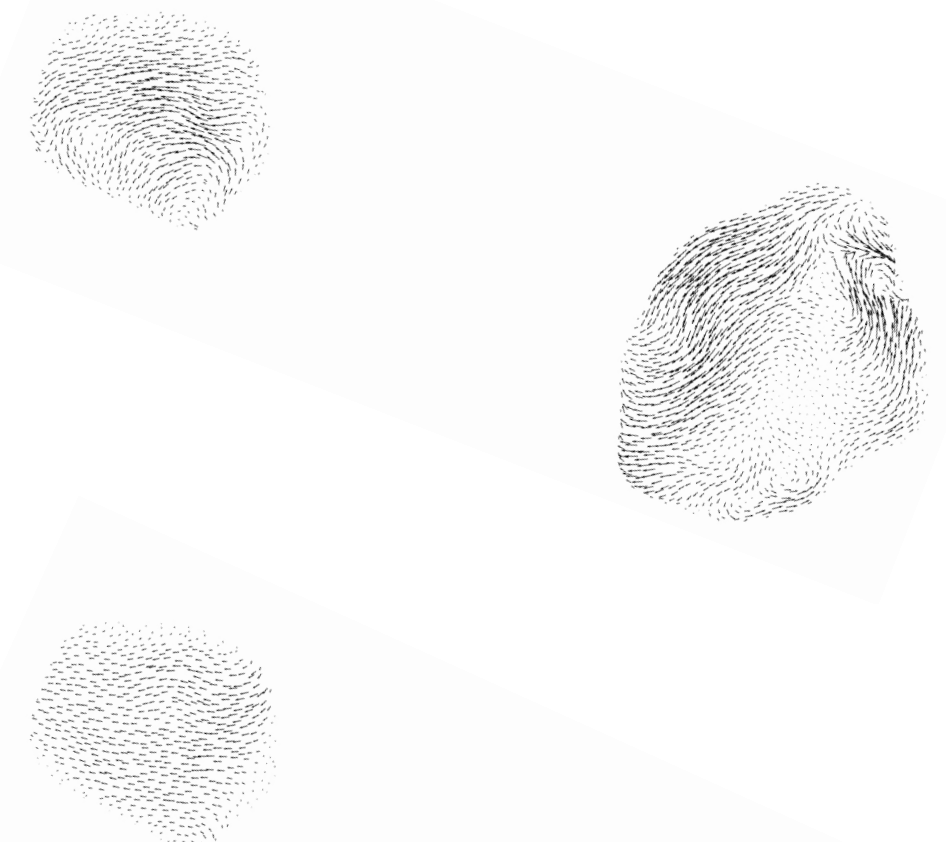

$\frac{0}{0}$

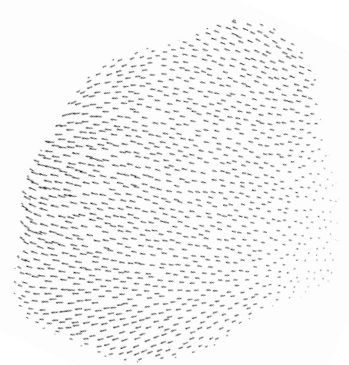

Figure 5.29: In plane velocity vector maps of the aorta at early systole (point 1) computed with CFD, EMRI (Wall function) and measured with 4D Flow CMR in the transversal cross sections of the aortic arch (Figure 5.10 a). 

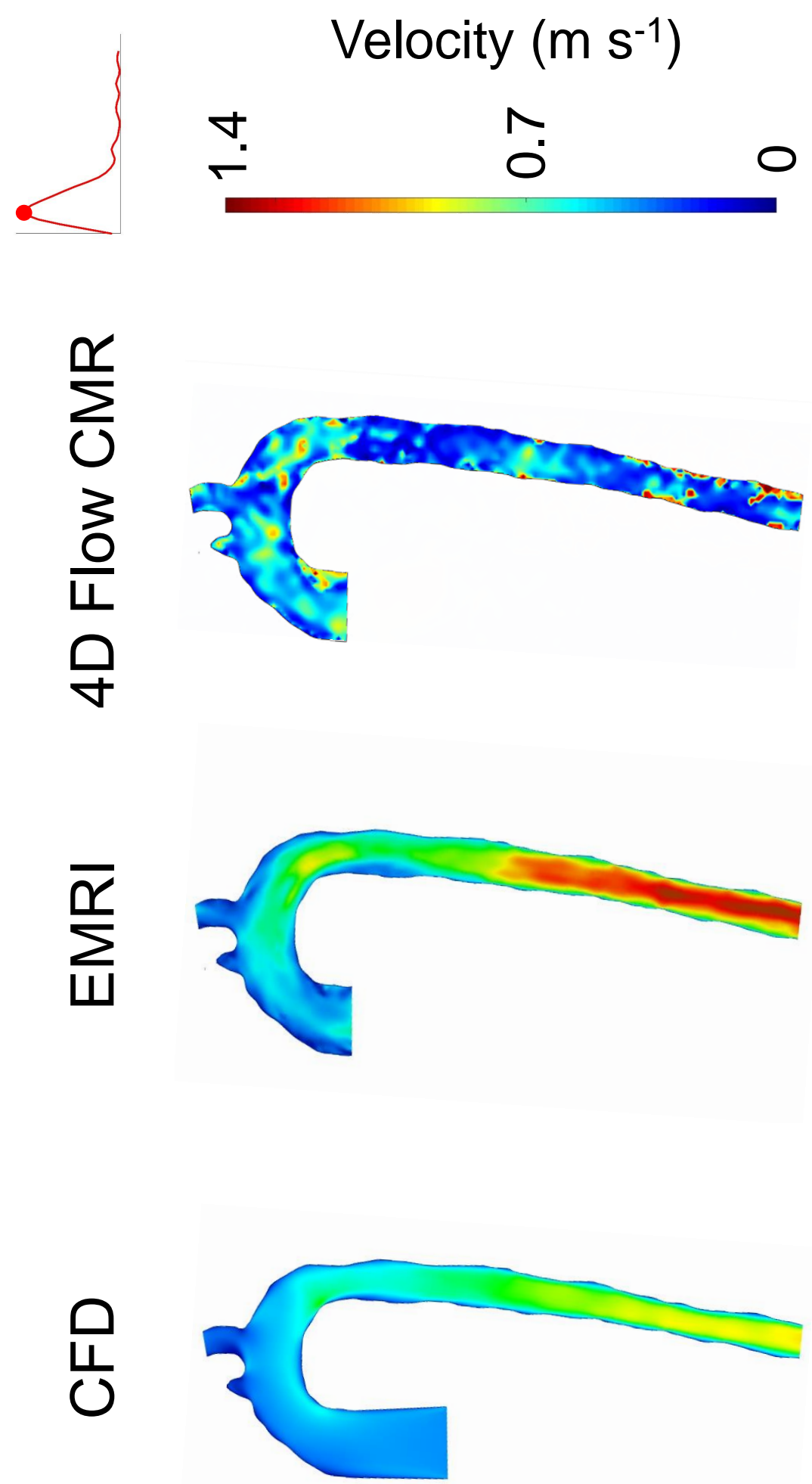

Figure 5.30: Velocity maps of the aorta at peak systole (point 2) computed with CFD, EMRI (Wall function) and measured with 4D Flow CMR in the sagittal cutting plane of the aorta (Figure $5.10 \mathrm{c}$ ). 

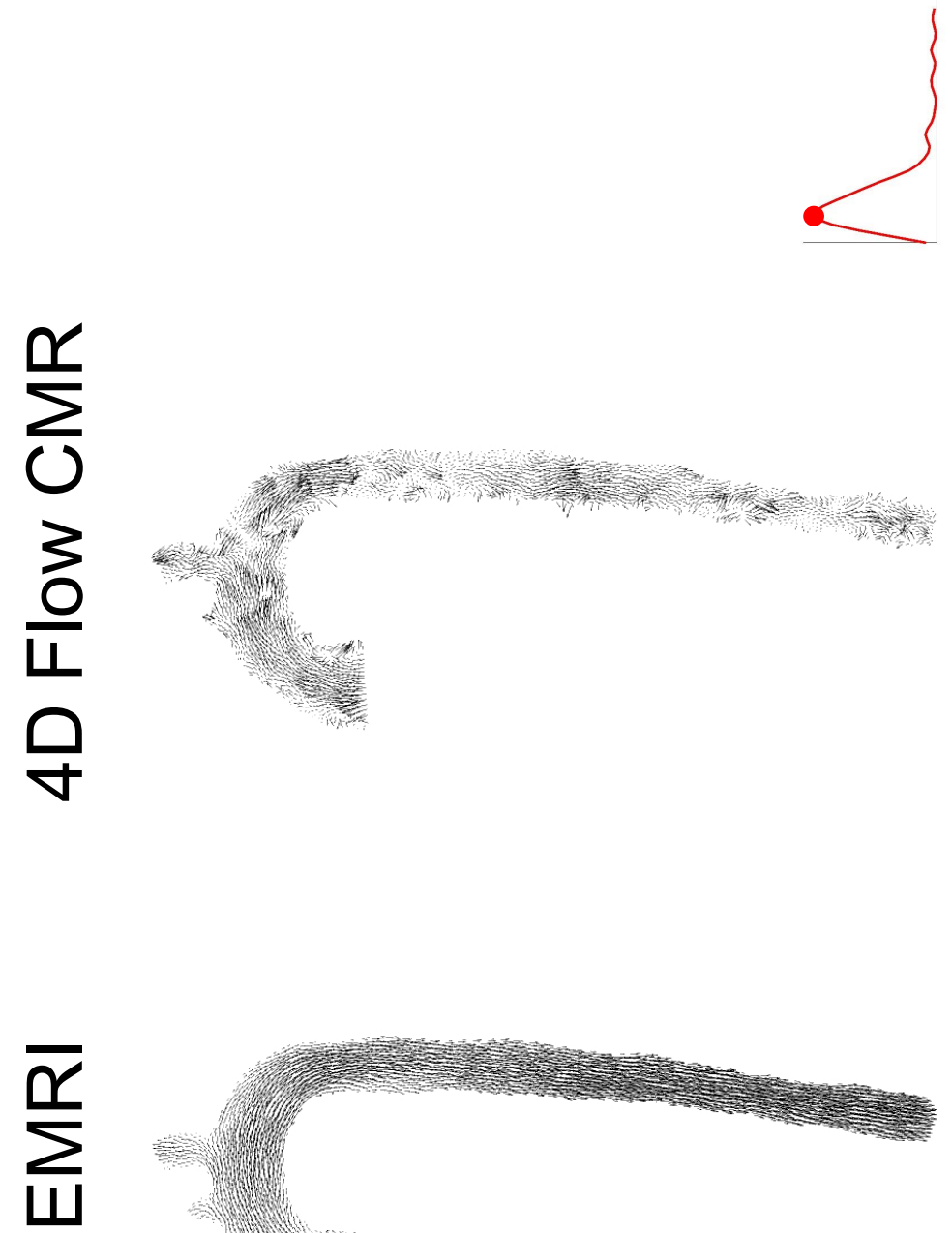

ш

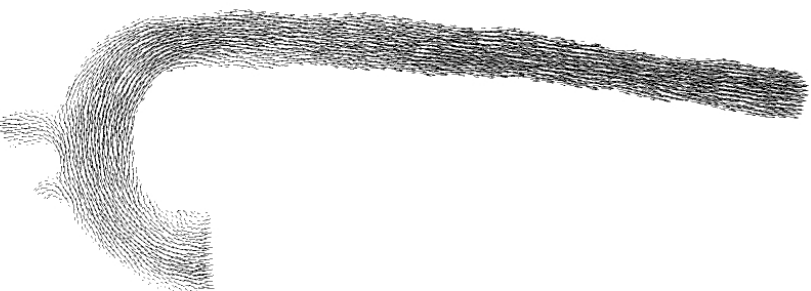

문

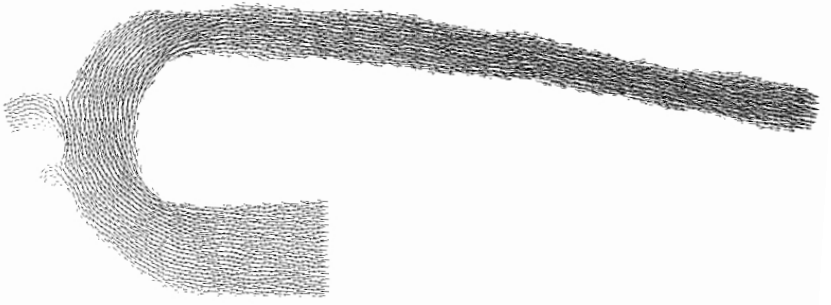

Figure 5.31: In plane velocity vector maps of the aorta at peak systole (point 2) computed with CFD, EMRI (Wall function) and measured with 4D Flow CMR in the sagittal cutting plane of the aorta (Figure $5.10 \mathrm{c}$ ). 

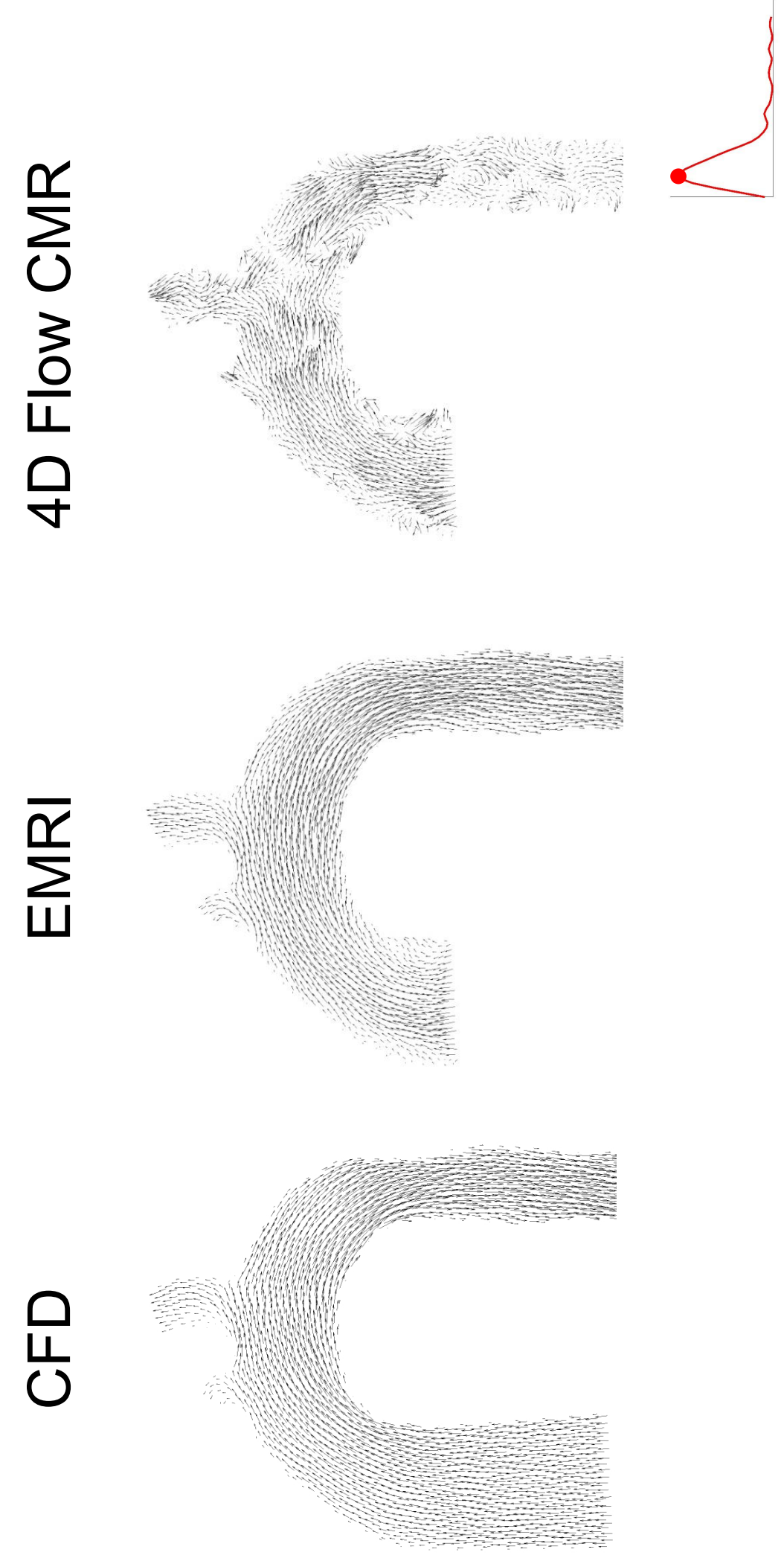

Figure 5.32: In plane velocity vector maps of the aortic arch at peak systole (point 2) computed with CFD, EMRI (Wall function) and measured with 4D Flow CMR in the sagittal cutting plane of the aorta (Figure $5.10 \mathrm{c}$ ). 
CFD

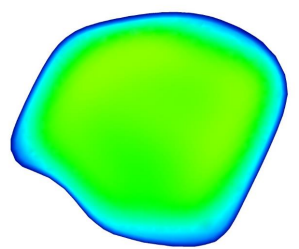

EMRI

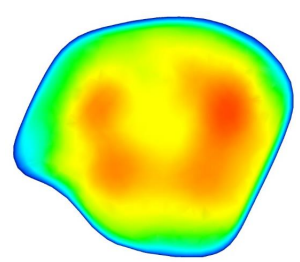

4D Flow CMR

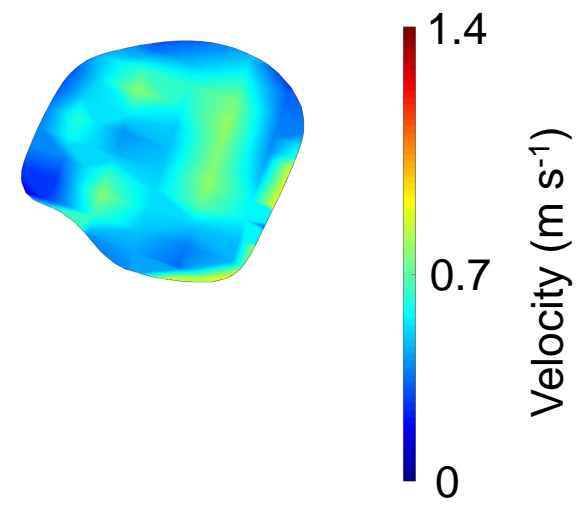

Figure 5.33: Velocity maps of the aorta at peak systole (point 2) computed with CFD, EMRI (Wall function) and measured with 4D Flow CMR in the transversal cross section of the aorta (Figure $5.10 \mathrm{~b}$ ).

CFD

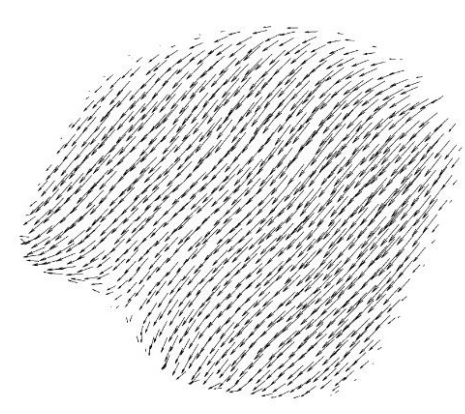

EMRI

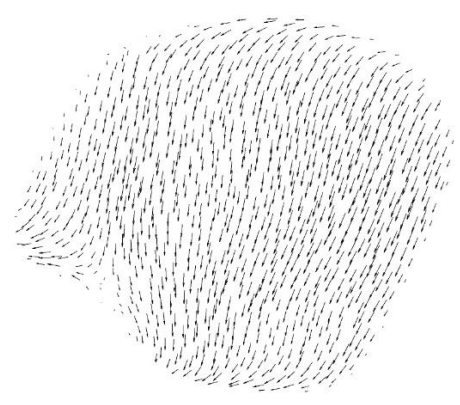

4D Flow CMR

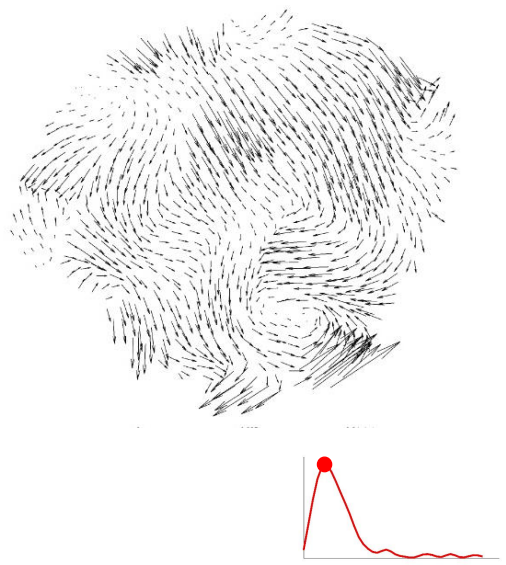

Figure 5.34: In plane velocity vector maps of the aorta at peak systole (point 2) computed with CFD, EMRI (Wall function) and measured with 4D Flow CMR in the transversal cross section of the aorta (Figure $5.10 \mathrm{~b}$ ). 

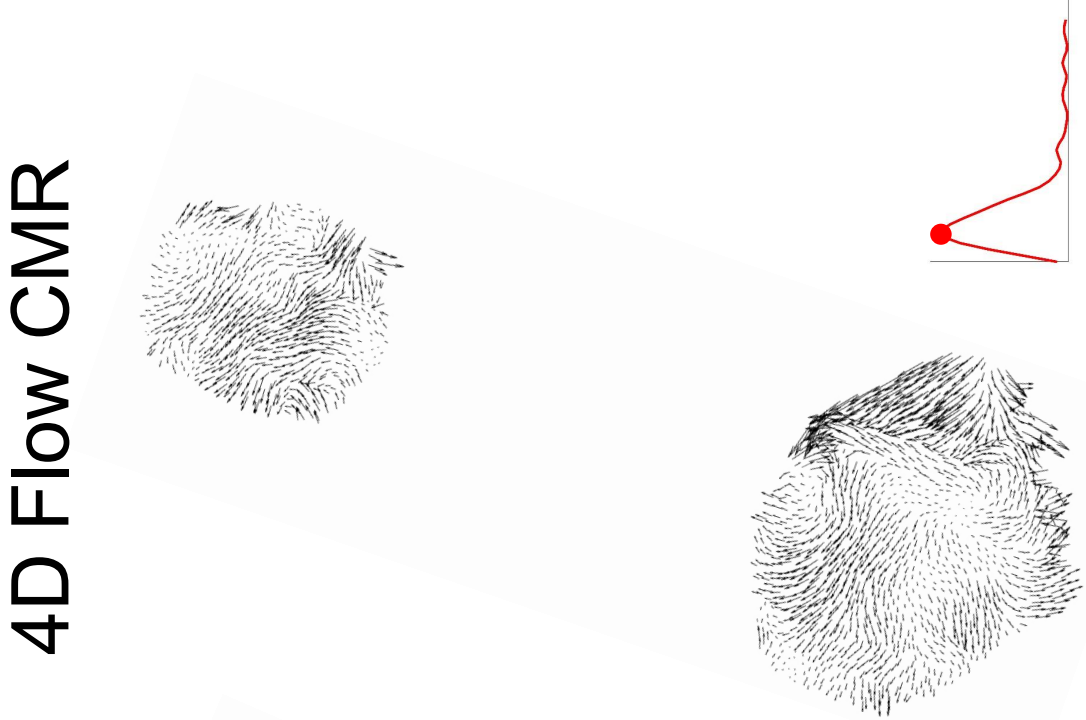

$\sum_{\dot{w}}^{\bar{\alpha}}$
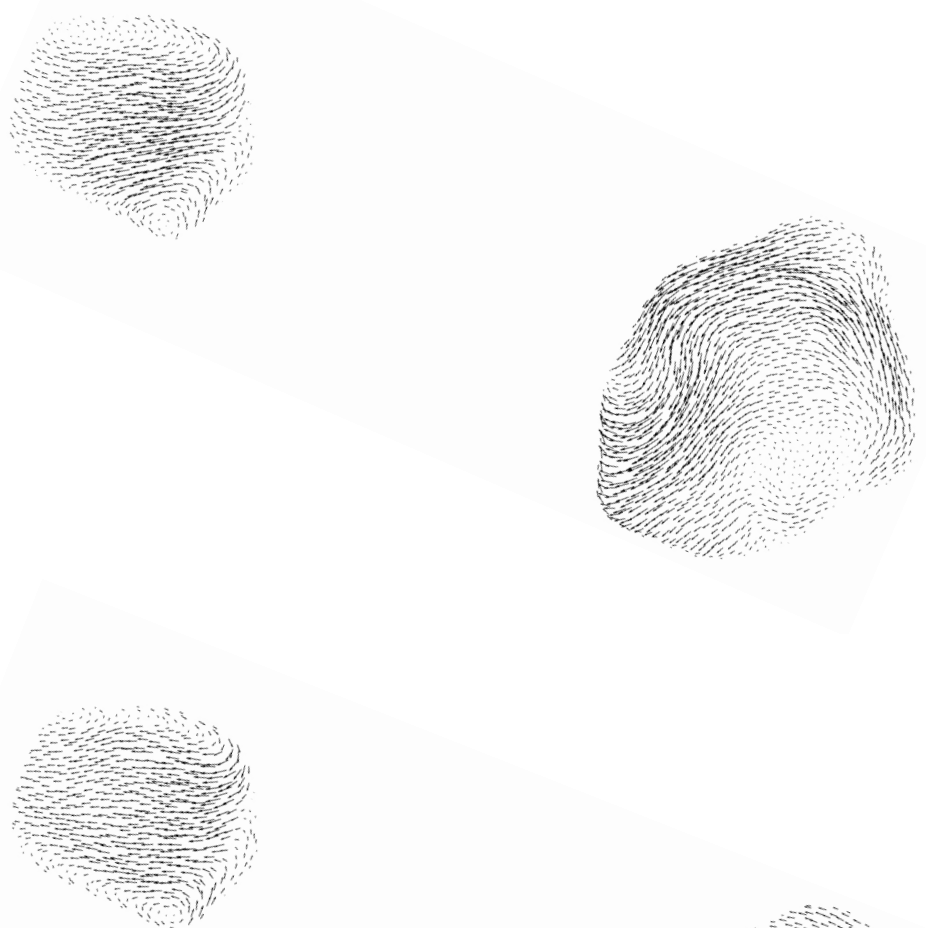

㞻

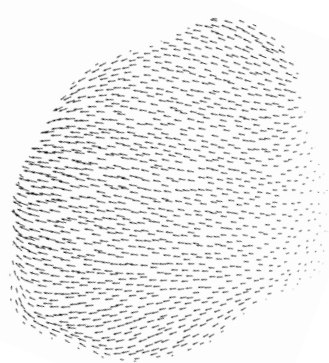

Figure 5.35: In plane velocity vector maps of the aorta at peak systole (point 2) computed with CFD, EMRI (Wall function) and measured with 4D Flow CMR in the transversal cross sections of the aortic arch (Figure 5.10 a). 

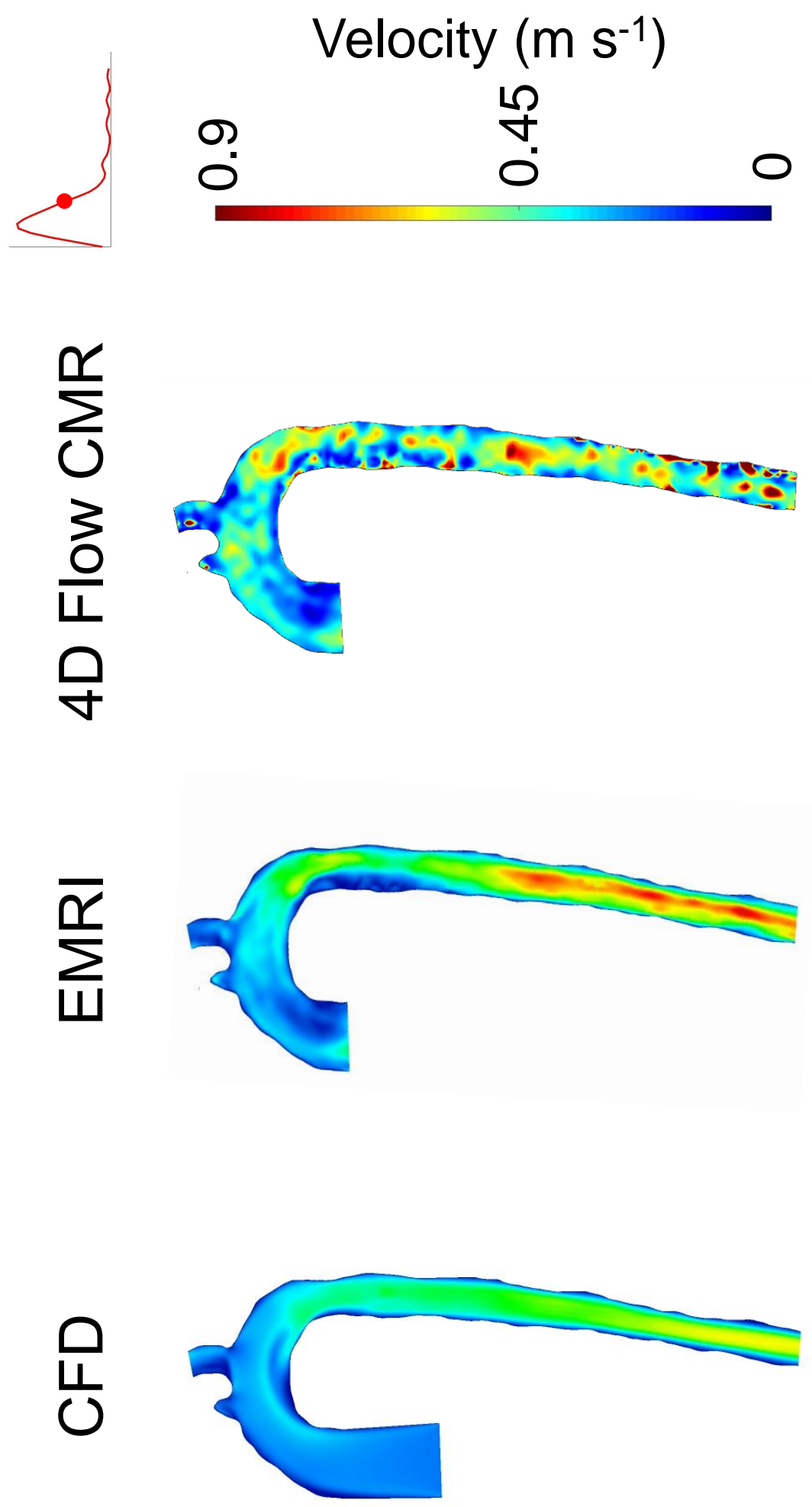

Figure 5.36: Velocity maps of the aorta at late systole (point 3 ) computed with CFD, EMRI (Wall function) and measured with 4D Flow CMR in the sagittal cutting plane of the aorta (Figure $5.10 \mathrm{c}$ ). 

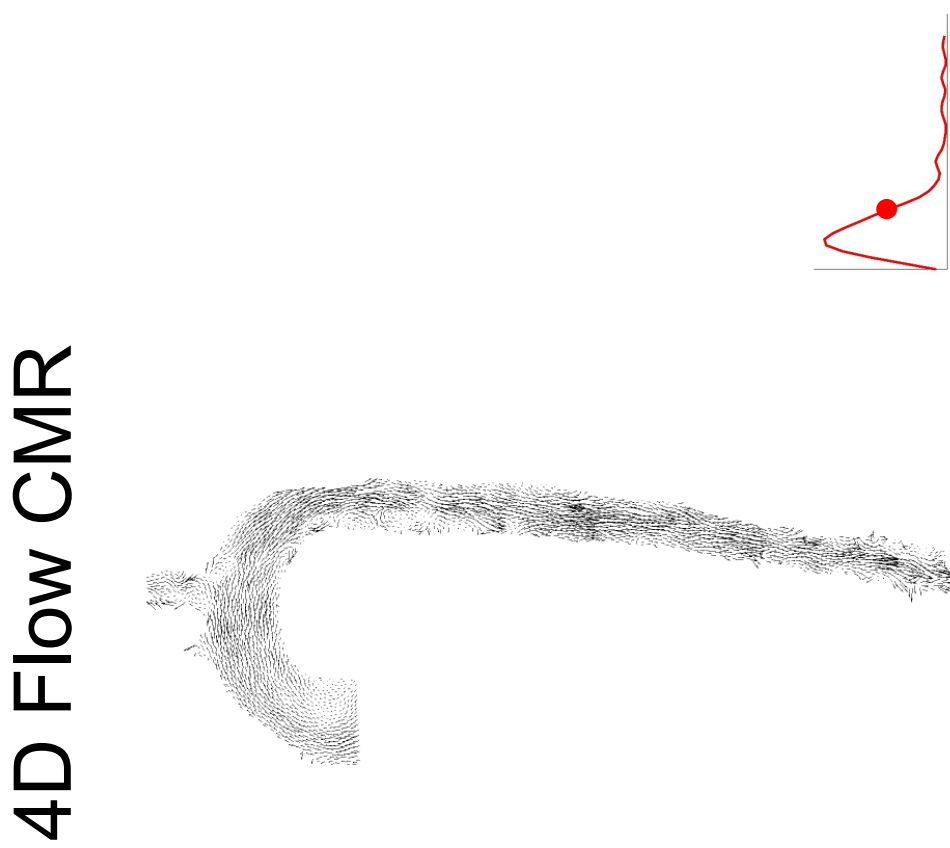

$\sum_{\dot{W}}^{\bar{\alpha}}$

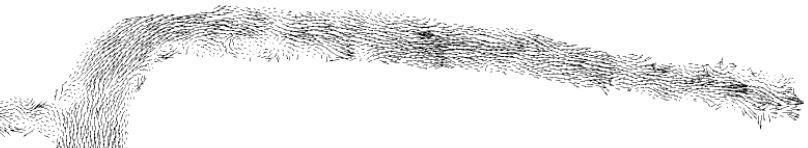

丩

$\nabla$

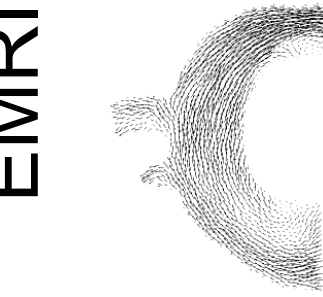

눈

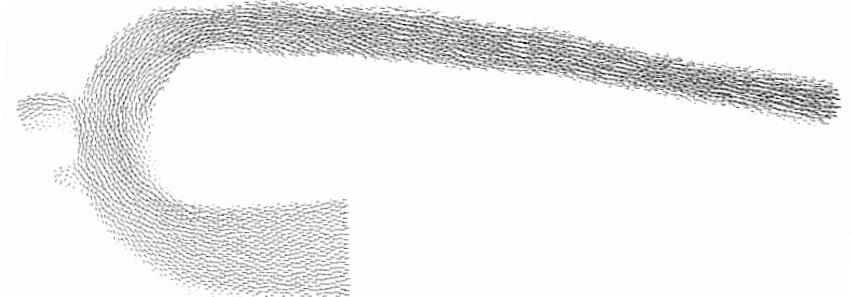

Figure 5.37: In plane velocity vector maps of the aorta at late systole (point 3) computed with CFD, EMRI (Wall function) and measured with 4D Flow CMR in the sagittal cutting plane of the aorta (Figure $5.10 \mathrm{c}$ ). 

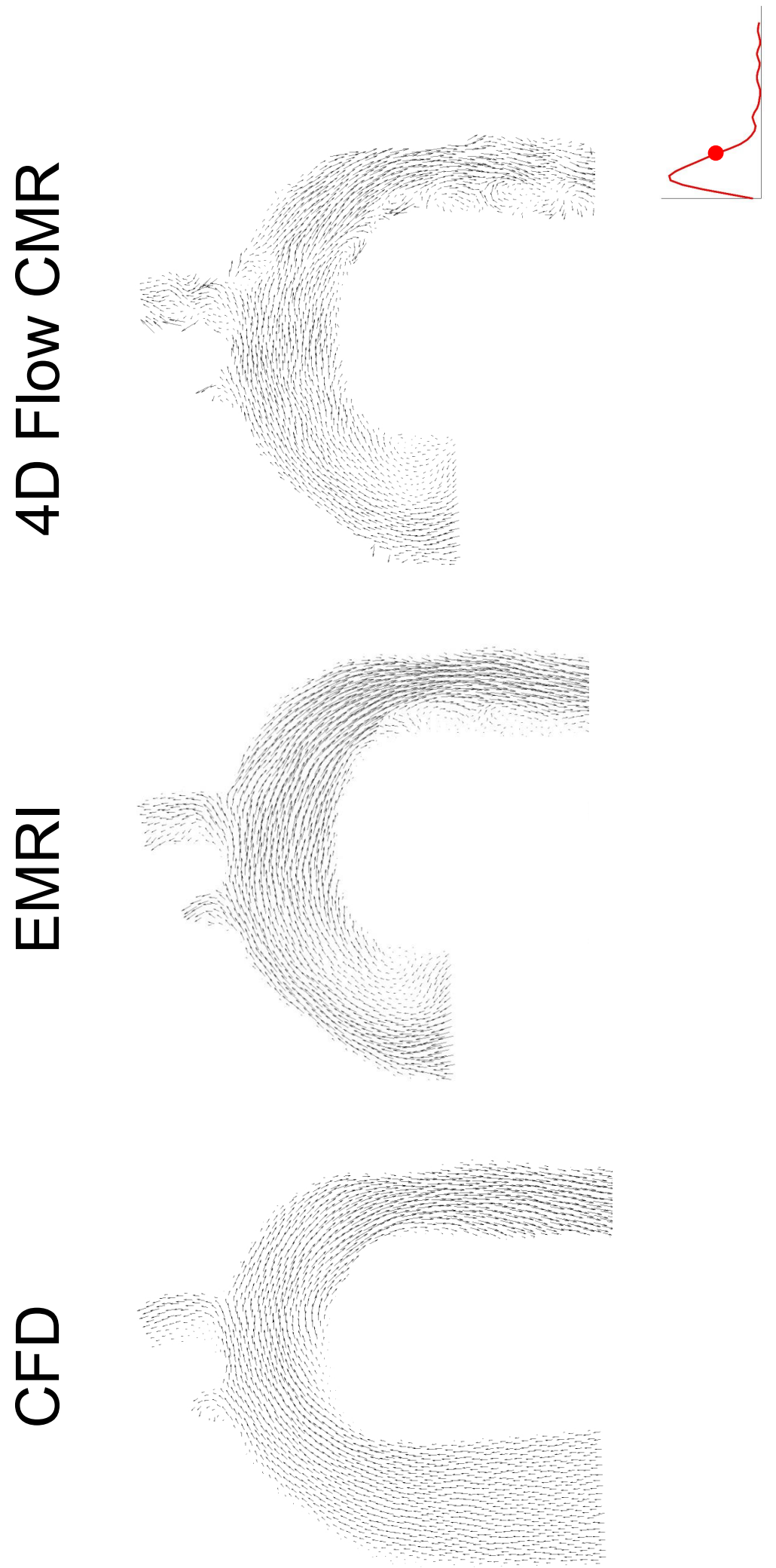

Figure 5.38: In plane velocity vector maps of the aortic arch at late systole (point 3) computed with CFD, EMRI (Wall function) and measured with 4D Flow CMR in the sagittal cutting plane of the aorta (Figure $5.10 \mathrm{c}$ ). 
CFD

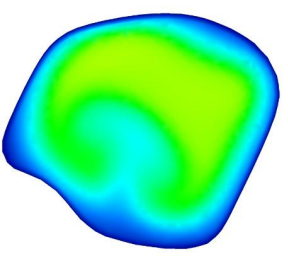

EMRI

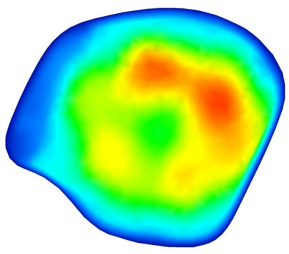

4D Flow CMR

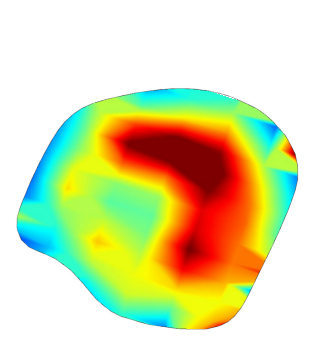

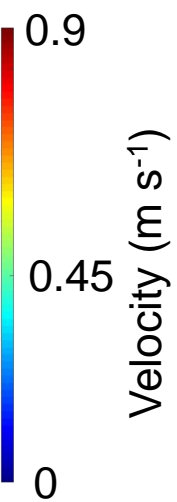

Figure 5.39: Velocity maps of the aorta at late systole (point 3 ) computed with CFD, EMRI (Wall function) and measured with 4D Flow CMR in the transversal cross section of the aorta (Figure $5.10 \mathrm{~b}$ ).

CFD

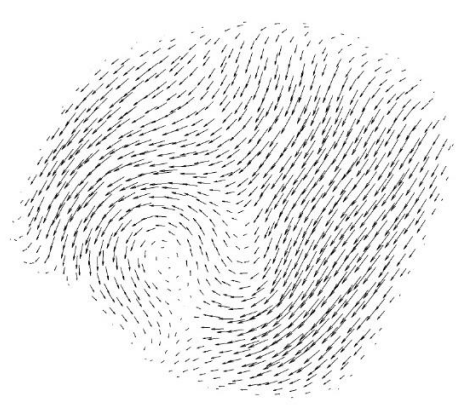

\section{EMRI}

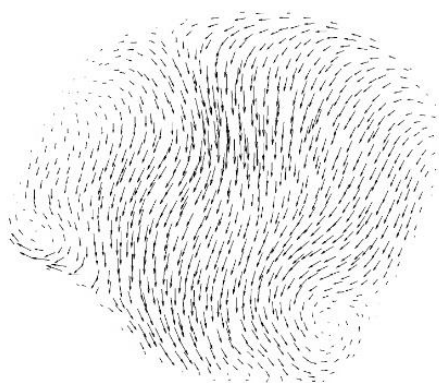

4D Flow CMR

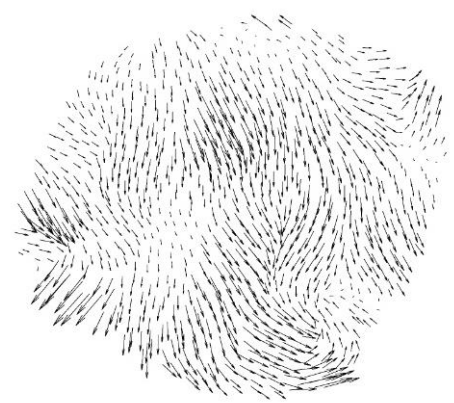

Figure 5.40: In plane velocity vector maps of the aorta at late systole (point 3) computed with CFD, EMRI (Wall function) and measured with 4D Flow CMR in the transversal cross section of the aorta (Figure $5.10 \mathrm{~b}$ ). 

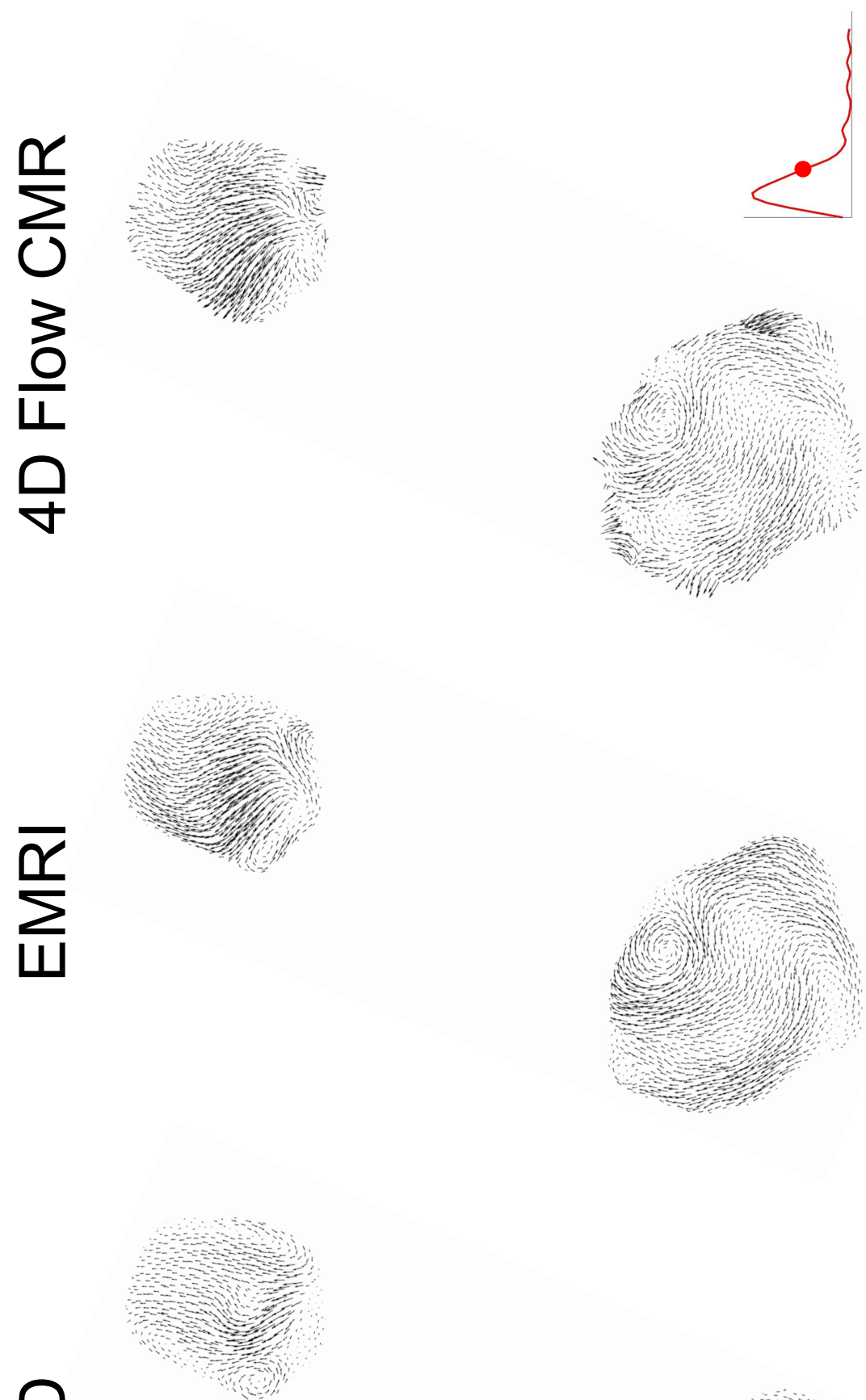

过

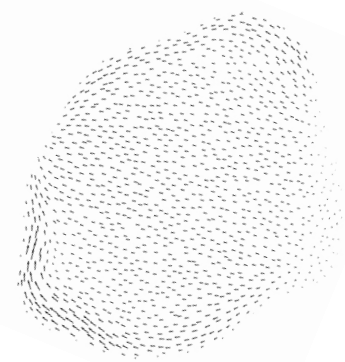

Figure 5.41: In plane velocity vector maps of the aorta at late systole (point 3 ) computed with CFD, EMRI (Wall function) and measured with 4D Flow CMR in the transversal cross sections of the aortic arch (Figure 5.10 a). 
Velocity $\left(\mathrm{m} \mathrm{s}^{-1}\right)$
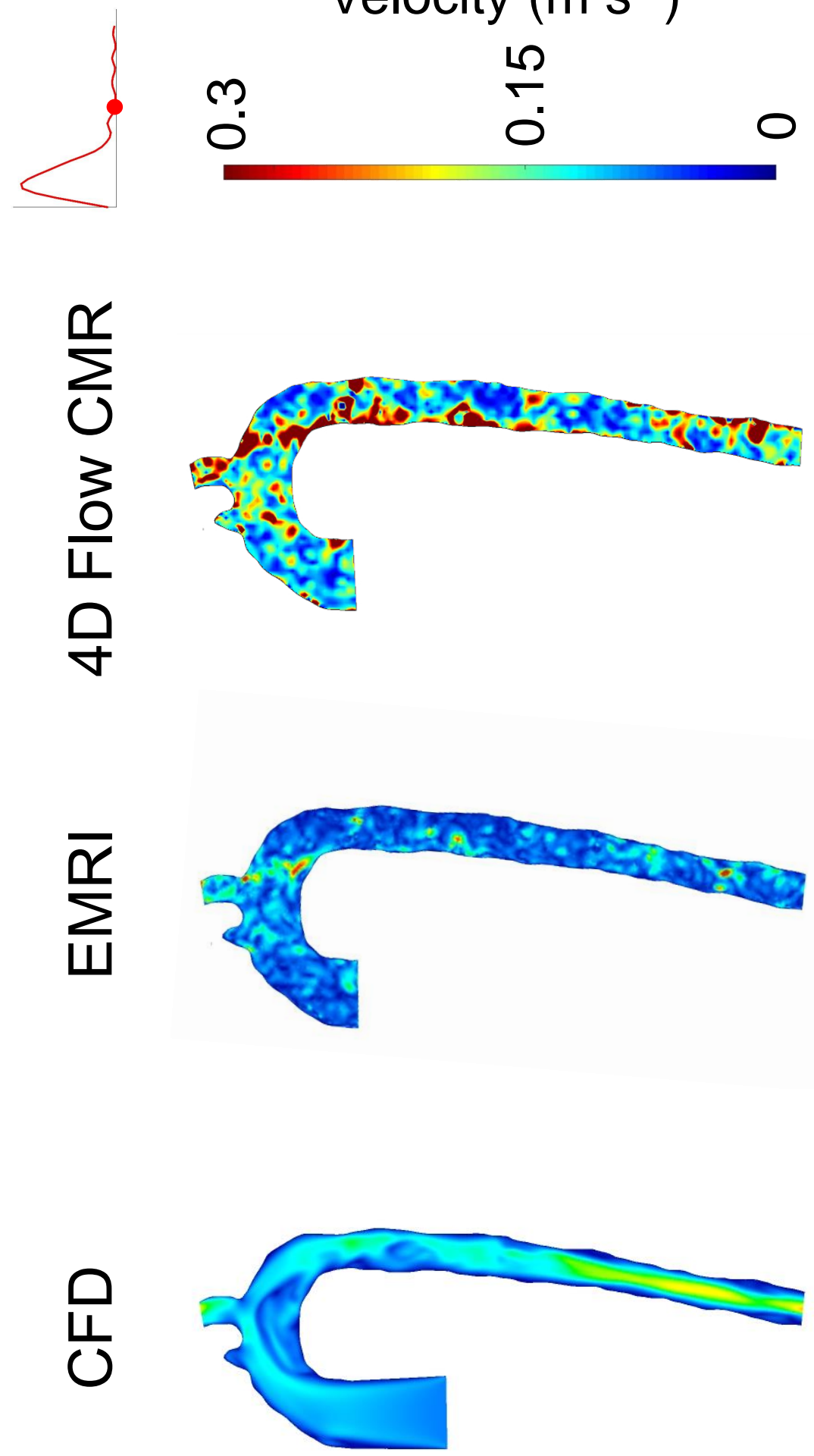

Figure 5.42: Velocity maps of the aorta at mid diastole (point 4) computed with CFD, EMRI (Wall function) and measured with 4D Flow CMR in the sagittal cutting plane of the aorta (Figure $5.10 \mathrm{c}$ ). 

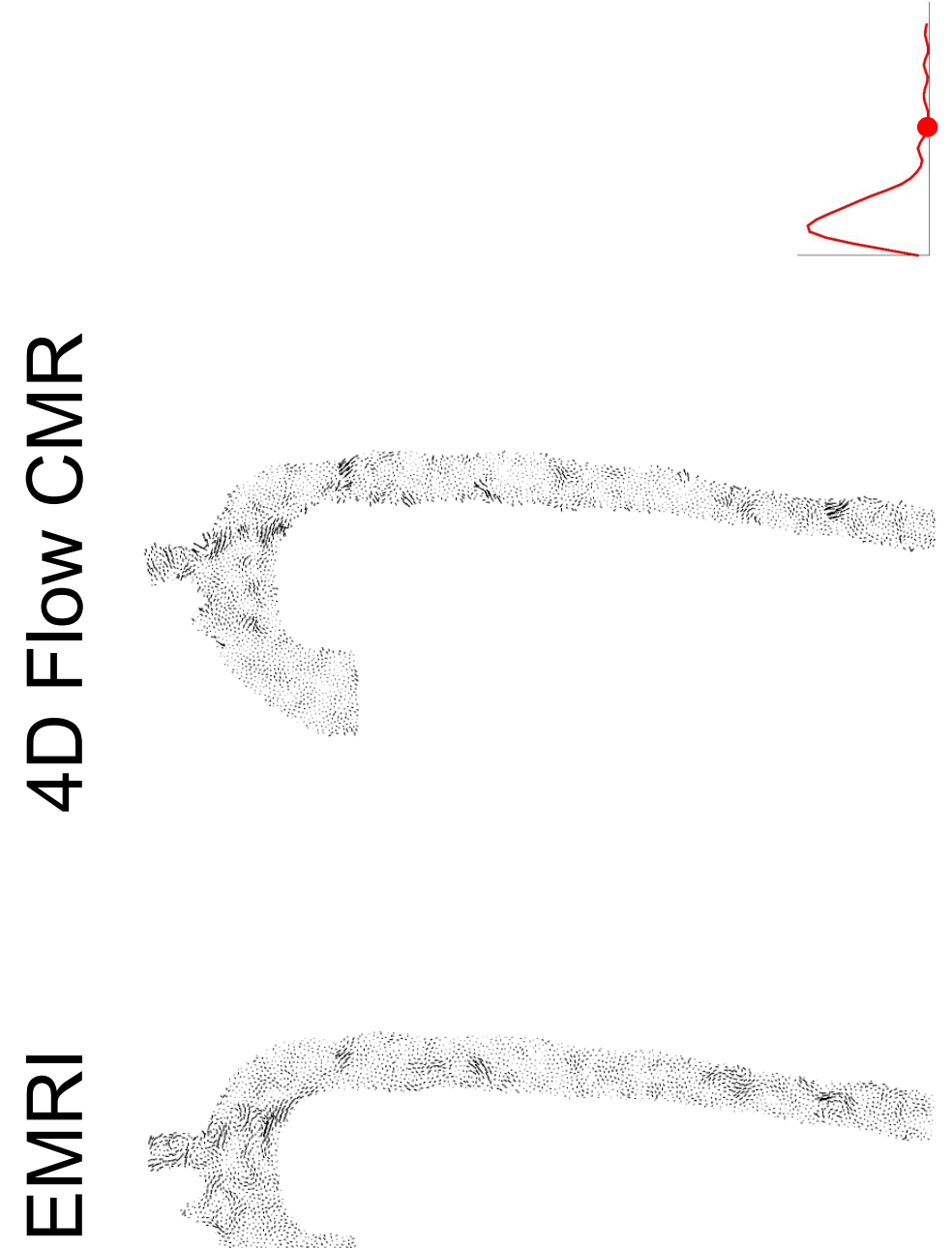

눈

Figure 5.43: In plane velocity vector maps of the aorta at mid diastole (point 4) computed with CFD, EMRI (Wall function) and measured with 4D Flow CMR in the sagittal cutting plane of the aorta (Figure $5.10 \mathrm{c}$ ). 

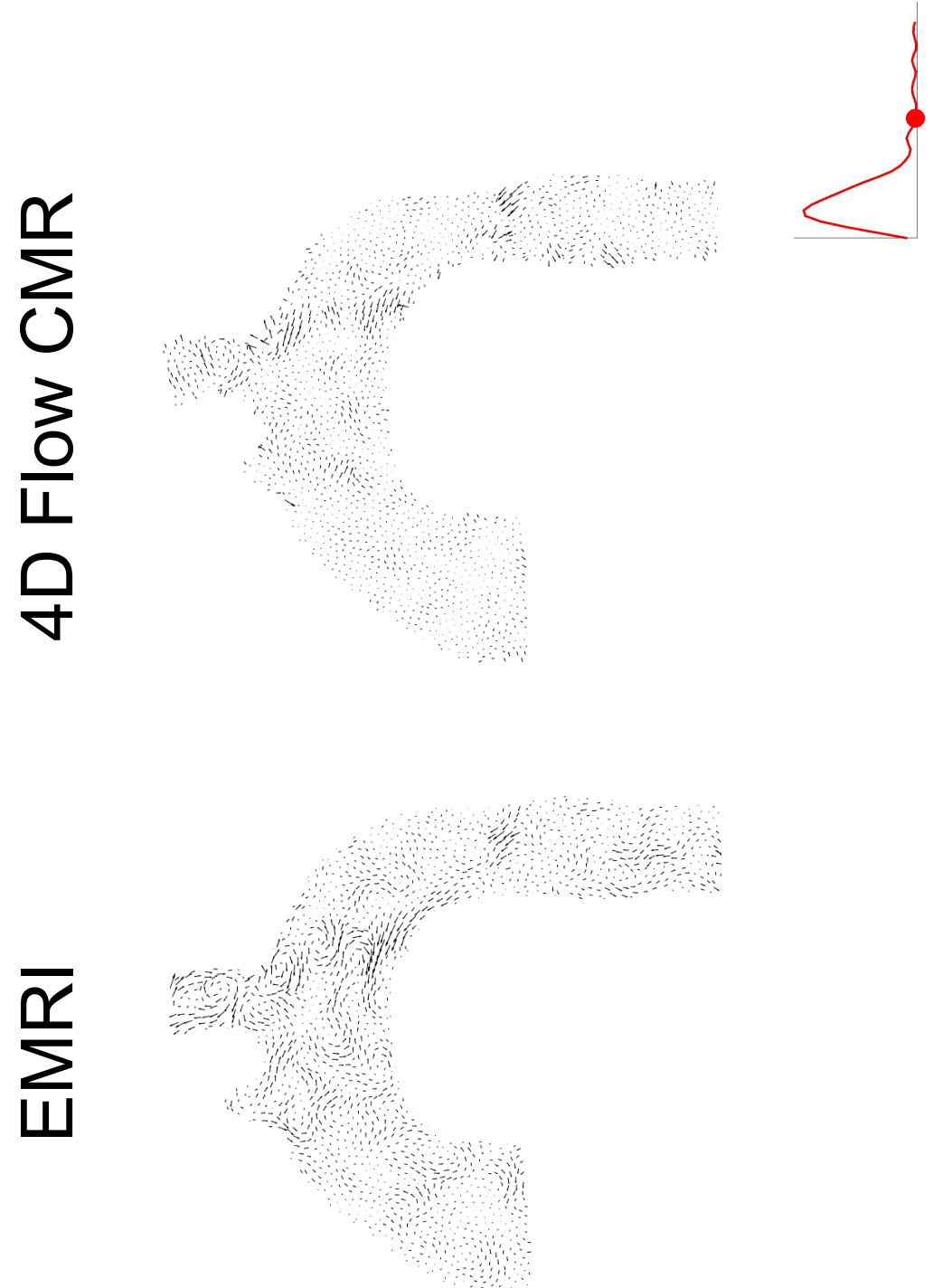

0

ว

ㄴ.

$\nabla$ 


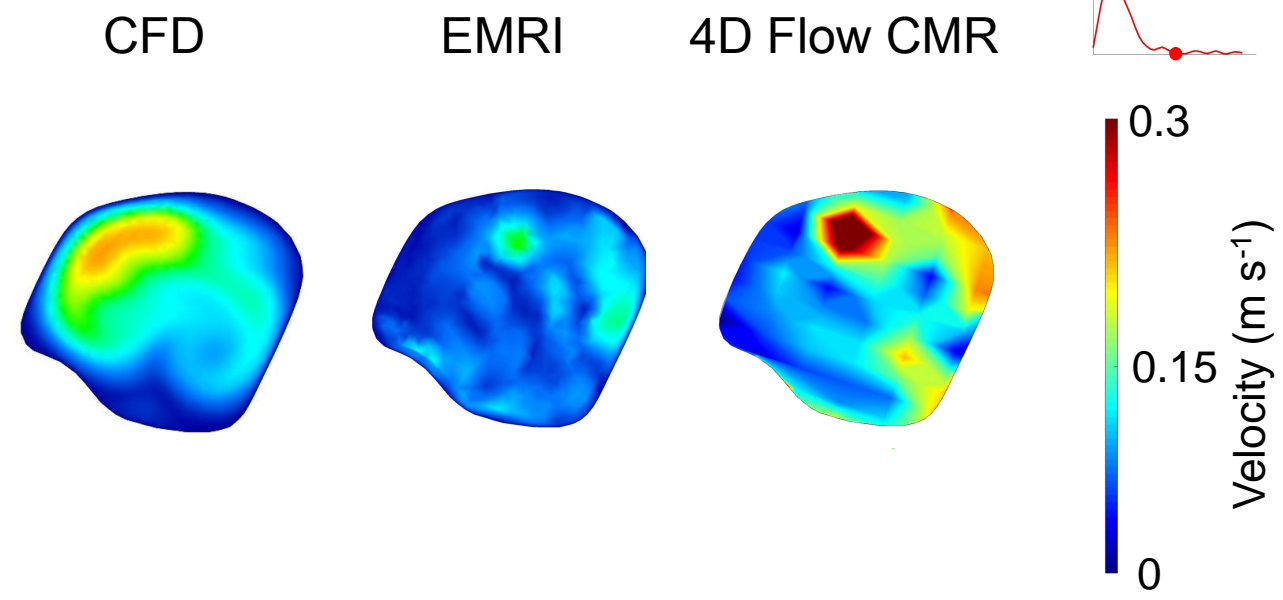

Figure 5.45: Velocity maps of the aorta at mid diastole (point 4) computed with CFD, EMRI (Wall function) and measured with 4D Flow CMR in the transversal cross section of the aorta (Figure $5.10 \mathrm{~b}$ ).

CFD

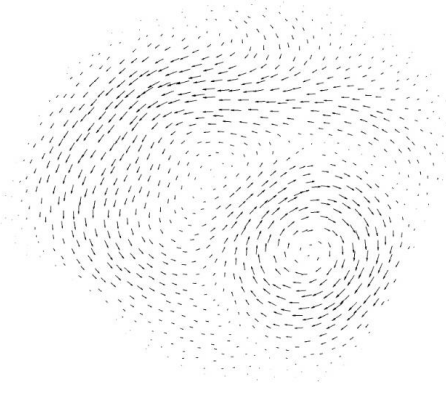

EMRI

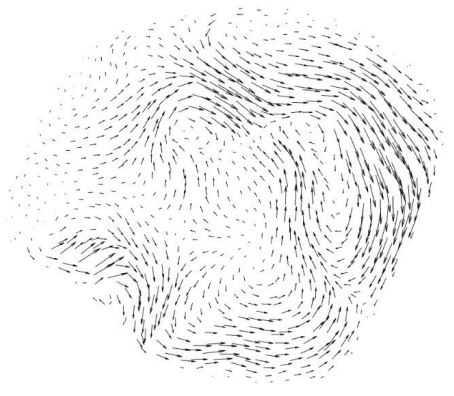

4D Flow CMR

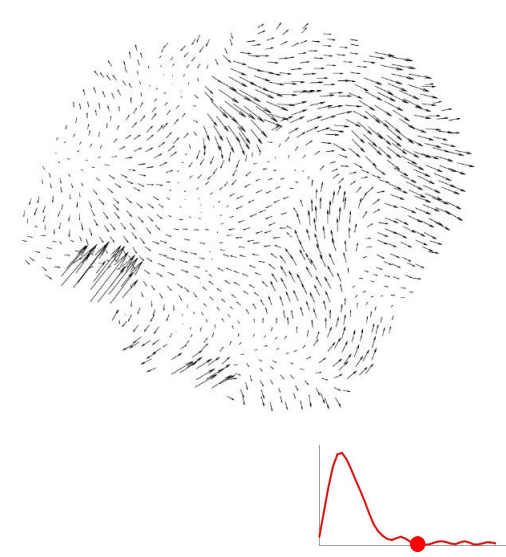

Figure 5.46: In plane velocity vector maps of the aorta at mid diastole (point 4) computed with CFD, EMRI (Wall function) and measured with 4D Flow CMR in the transversal cross section of the aorta (Figure $5.10 \mathrm{~b}$ ). 

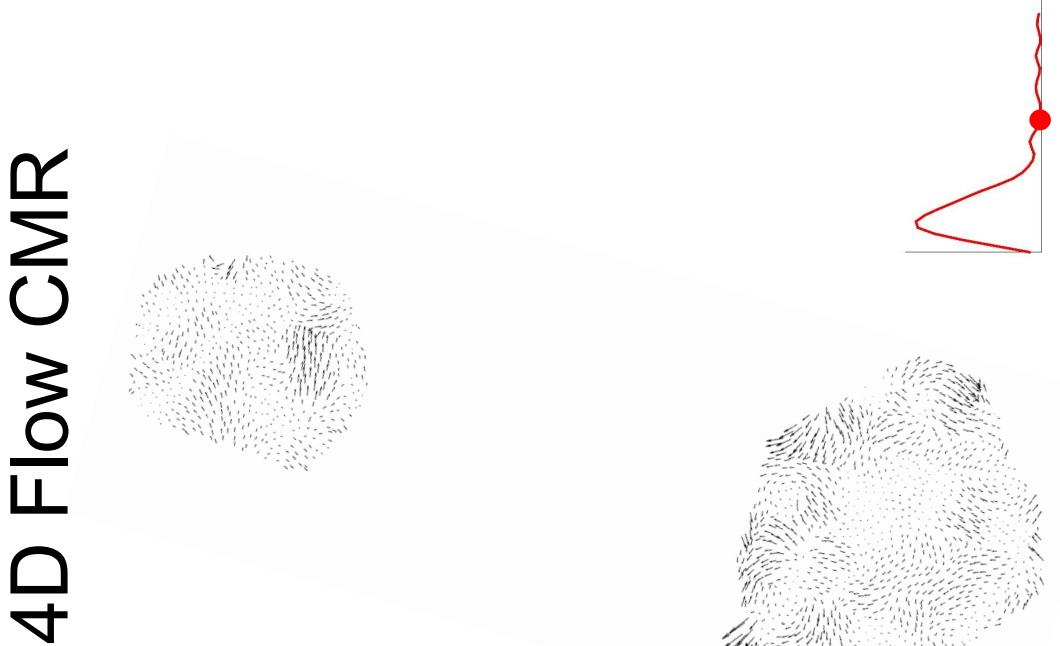

0

은

$\checkmark$
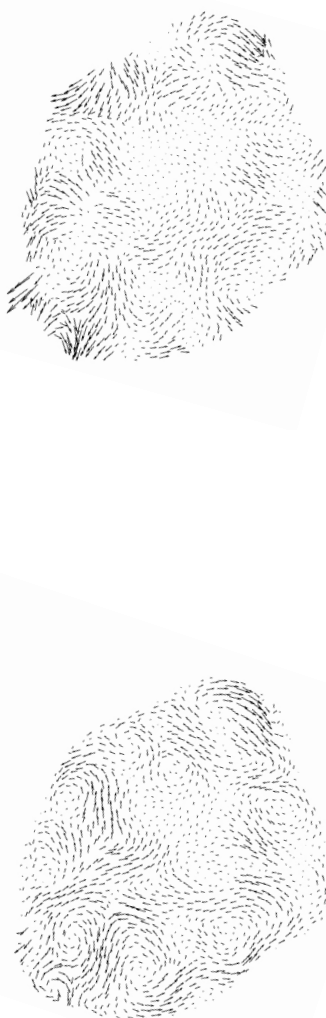

닌

Figure 5.47: In plane velocity vector maps of the aorta at mid diastole (point 4) computed with CFD, EMRI (Wall function) and measured with 4D Flow CMR in the transversal cross sections of the aortic arch (Figure 5.10 a). 

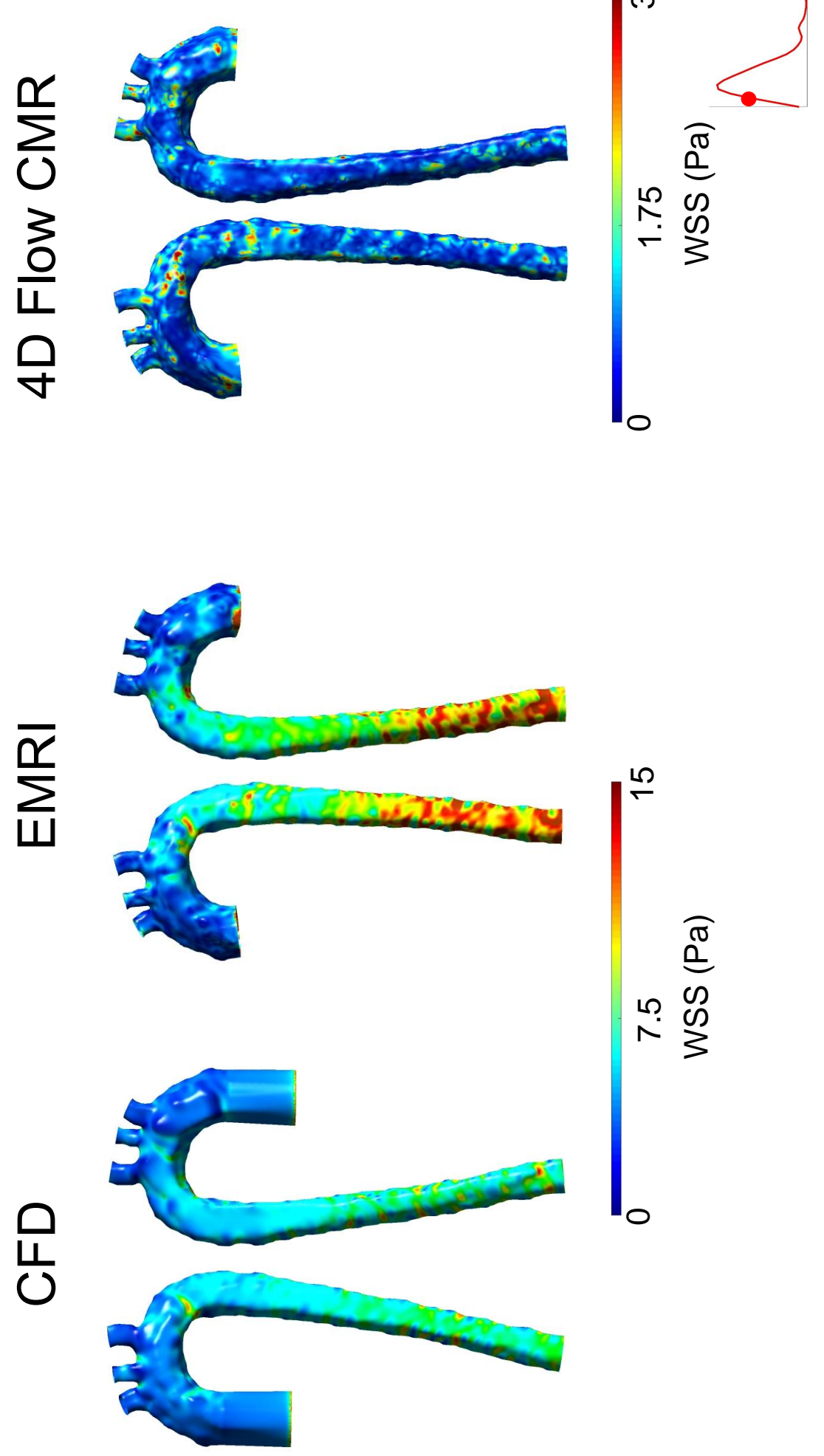

Figure 5.48: WSS maps of the two sides of the aorta at early systole (point 1) computed with CFD, EMRI (Wall function) and computed with 4D Flow CMR. 

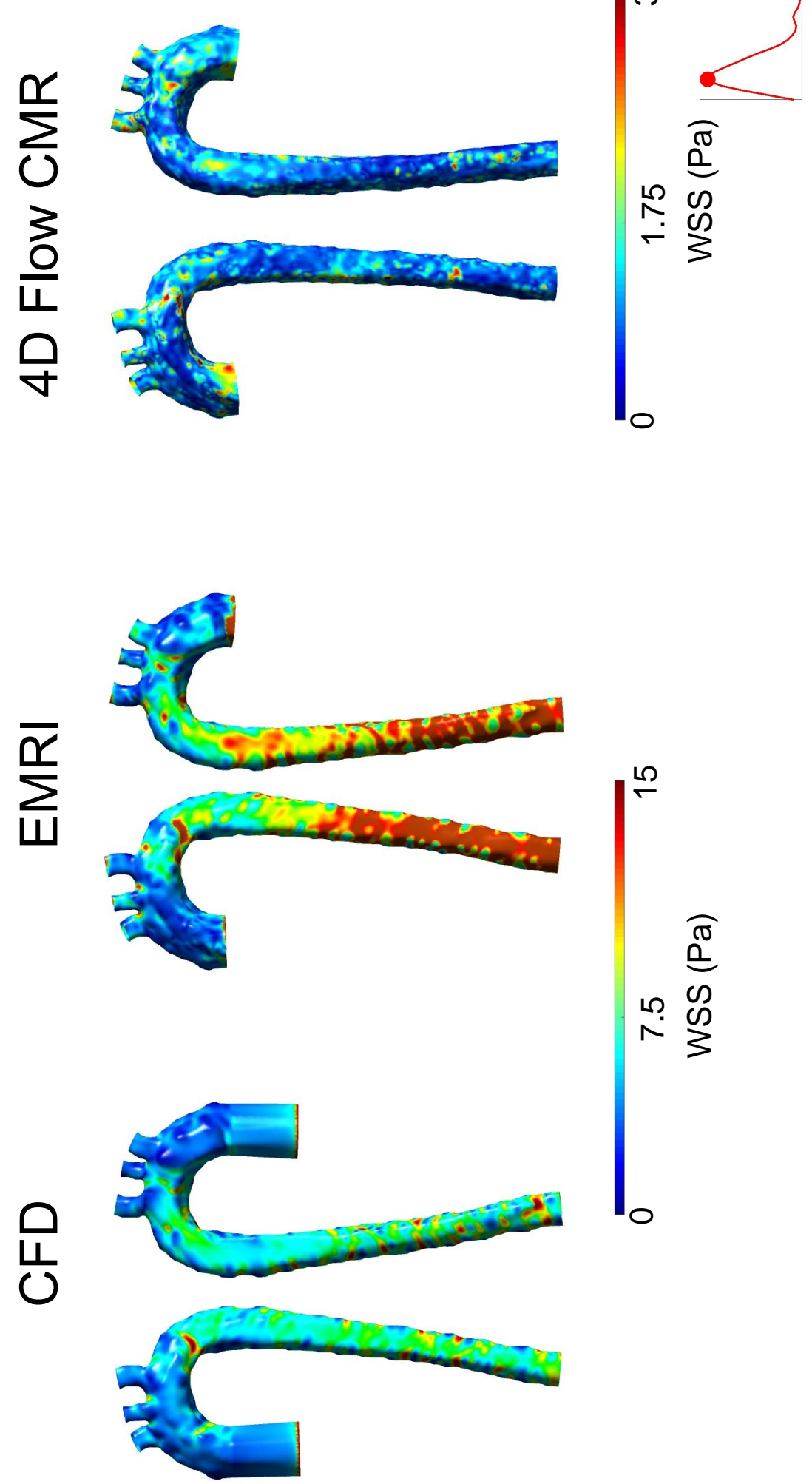

Figure 5.49: WSS maps of the two sides of the aorta at peak systole (point 2) computed with CFD, EMRI (Wall function) and computed with 4D Flow CMR. 

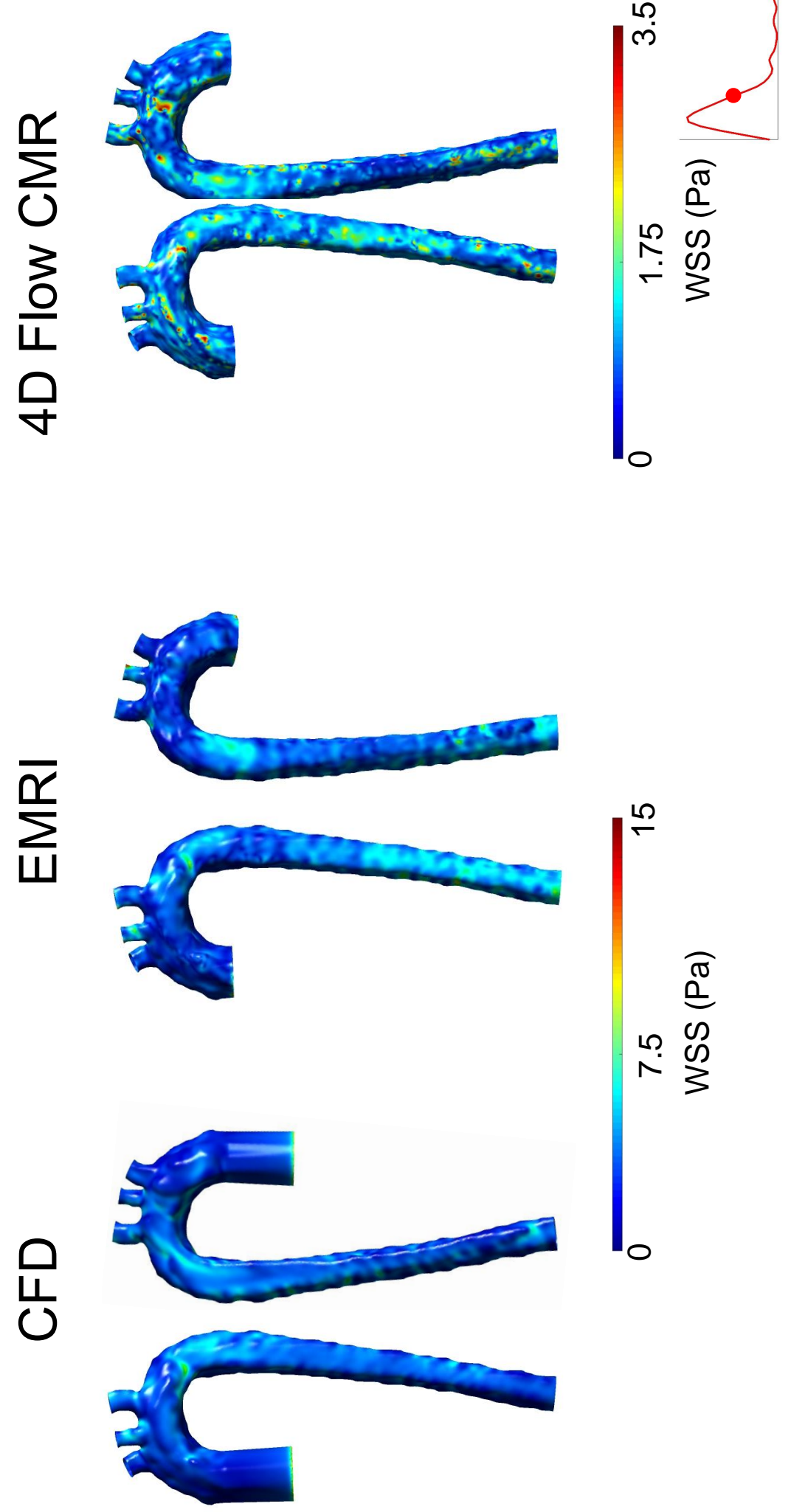

Figure 5.50: WSS maps of the two sides of the aorta at late systole (point 3) computed with CFD, EMRI (Wall function) and computed with 4D Flow CMR. 

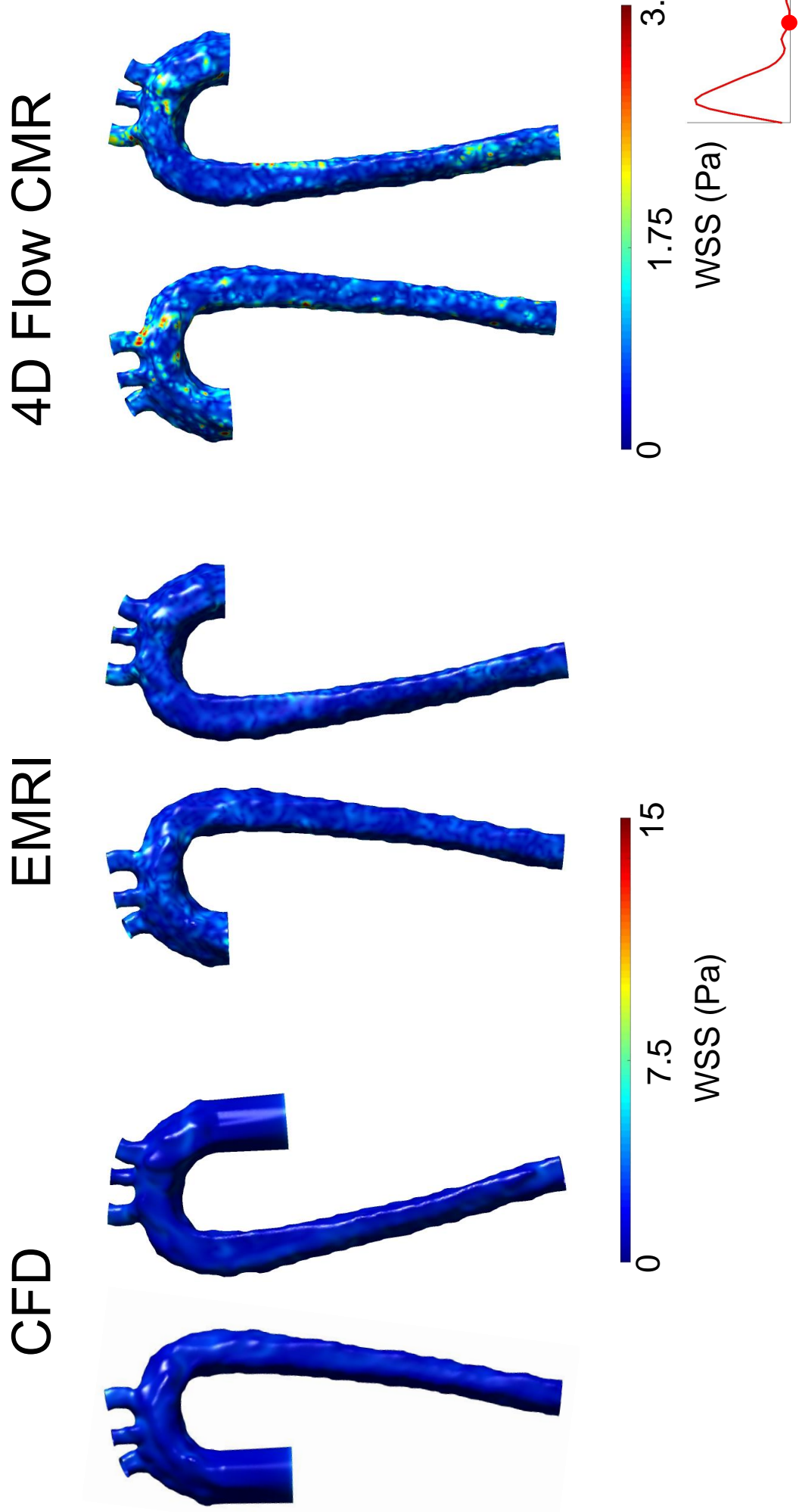

Figure 5.51: WSS maps of the two sides of the aorta at mid diastole (point 4) computed with CFD, EMRI (Wall function) and computed with 4D Flow CMR. 


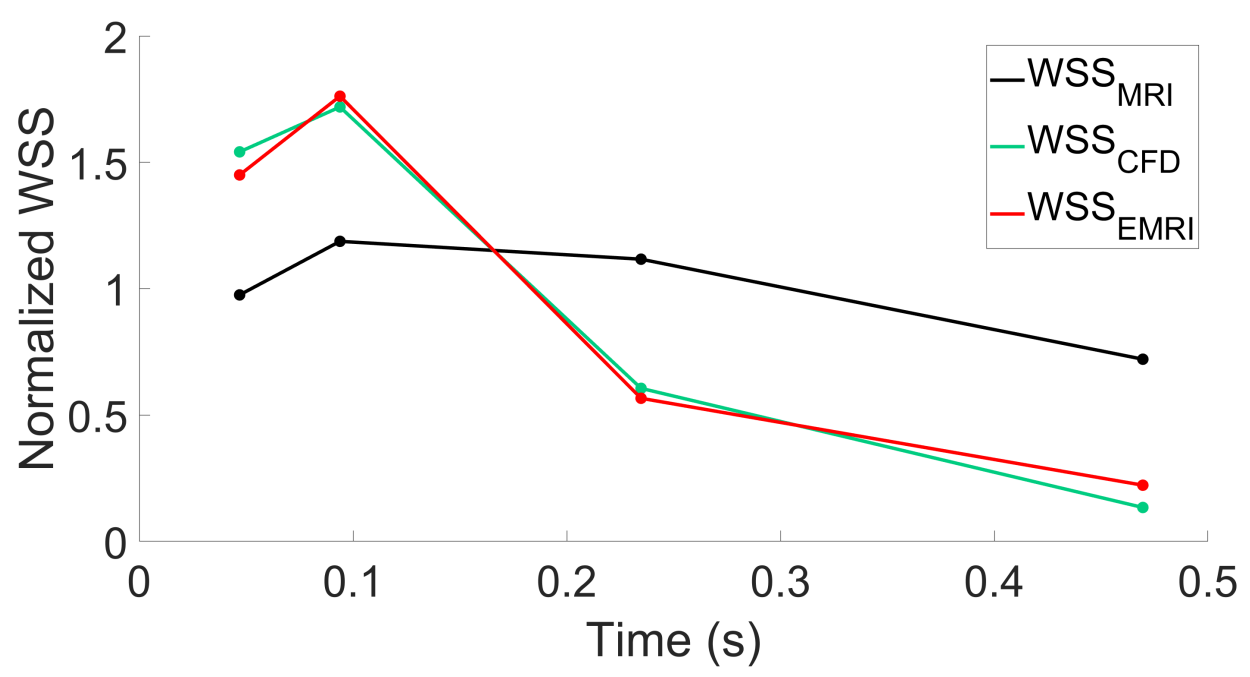

Figure 5.52: Normalized WSS retrieved from 4D Flow MRI, CFD and EMRI velocity data in the selected points of the cardiac cycle.
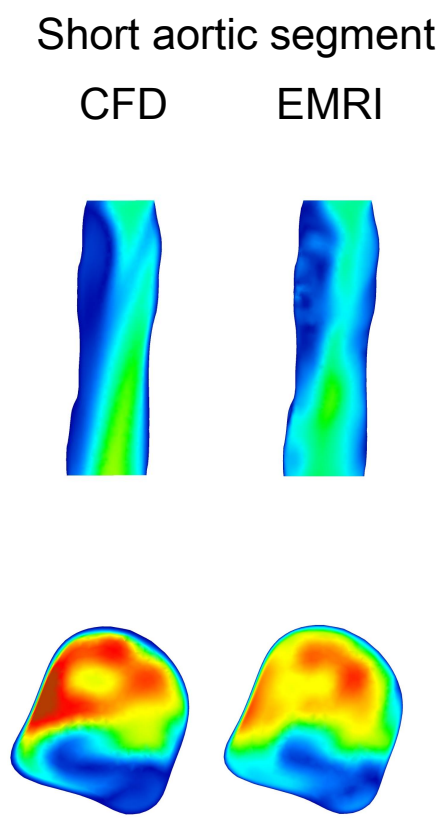
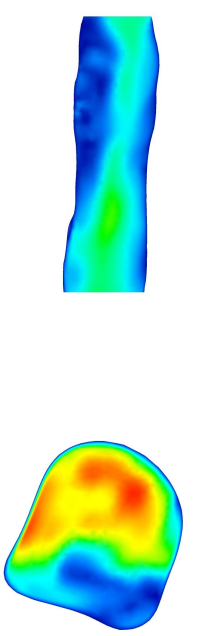
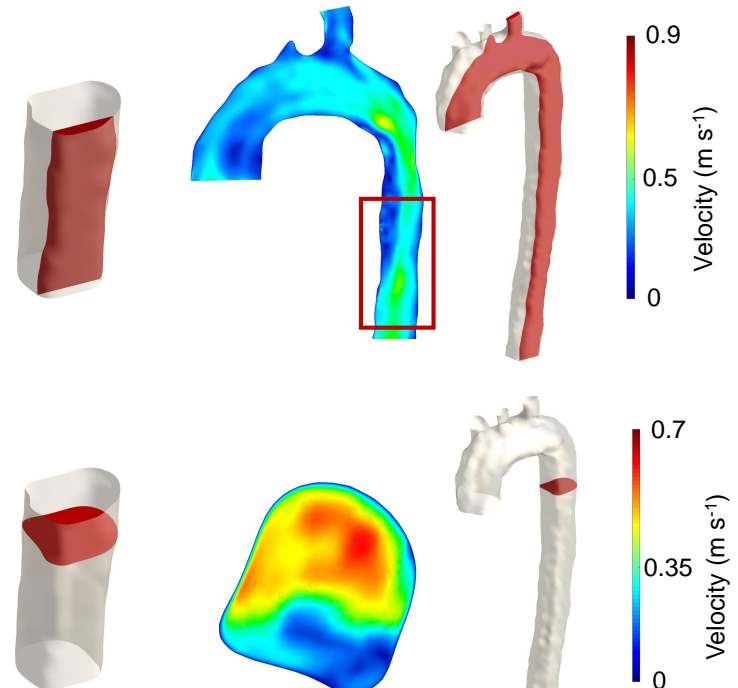

Entire aorta

EMRI

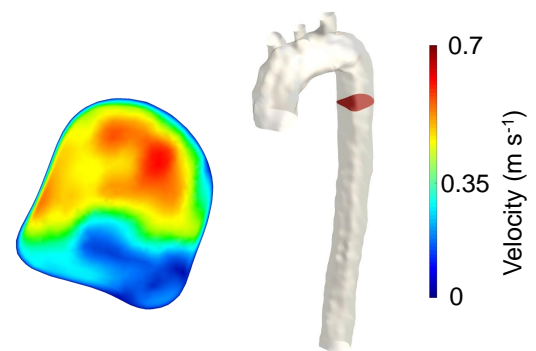

Figure 5.53: Velocity map of the longitudinal and cross-sectional planes of the aorta $(\mathrm{t}=0.2348 \mathrm{~s})$ computed for a short segment using standard CFD and EMRI, and entire aorta with EMRI 


\section{Chapter 6}

\section{Application of EMRI in pathophysiological research: atherogenesis}

\subsection{Background}

It has been more than a century that haemodynamic forces have been thought as the driving factors of vascular remodelling (the first study by Thoma dates 1893 [131]) and addressed as a trigger of vascular pathologies, particularly atherosclerosis [132] and is suggested as a biomarker to assess risk factor of cardiovascular events (e.g. stroke).

In the atherosclerosis field, WSS, the frictional force exerted per unit area by the flow of blood on the vessel wall, has been one of the main marker. Two contradicting theories had been proposed to explain the distribution of the lesions [59].

Fry et al. [133] suggested high WSS is responsible for endothelial damage and the development of pathologies, while Caro et al.[132] proposed the low WSS to be responsible. The studies carried out in the subsequent three decades have seen the low WSS theory prevailing as the main responsible factor of plaque development [134].

Together with WSS, different biomarkers have been proposed, e.g. oscillatory shear index (OSI) [135], time averaged wall shear stress (TAWSS) [136]. 
The theory of low WSS has become widely accepted as 'primum movens' of atherosclerosis $[59,137]$, which can explain the focal nature of this disease.

In particular atherosclerosis involves the outer walls of vessel bifurcations and areas of flow recirculation or stagnation. In these regions WSS is drastically lower than in other areas which are not atheroprore. Computational studies found that atherosclerosis is likely to develop where WSS is $\pm 4 d y n e / \mathrm{cm}^{2}$ while, where WSS greater than 12 dyne $/ \mathrm{cm}^{2}$ the flow exerts an athero-protective stimulus [59]. Studies carried out on carotid bifurcations [138], coronary arteries [139] found a quantitative correlation between plaque localization, WSS and disturbed flow.

However as some studies highlighted, this theory is far from being perfect, especially when considering point-to-point correlation between WSS and atherosclerotic lesions (e.g. intimal, intima-medial, or wall thickening, lipid accumulation) $[134,140]$.

Moreover the WSS and transport phenomena of oxygen and macromolecules are related, but there is only qualitative evidence, and other factor such as vessel three-dimensionality also influence the concentration of macromolecules at the blood-wall interface and hence potentially macro molecule concentration within the wall [141].

WSS can be estimated from the velocity field measured in vivo using 4D Flow CMR [142, 39] or Doppler-ultrasound [143]. As discussed previously in this dissertation, the limited spatial and temporal resolutions could lead to errors in the estimated flow field, which in turn affects the accuracy of the MRI-retrieved parameters such as WSS [33].

As also pointed out, in the last two decades, CFD has been extensively used as a tool to simulate the flow patterns in different regions of the cardiovascular system, and used to asses WSS distributions. CFD demonstrated a good degree of accuracy when compared to in-vivo imaging modalities such as MRI [10]. SarramiForoushani et al. [69] studied the carotid bifurcation with 4D Flow CMR and CFD comparing the velocity vector field obtained by each technique and reporting a difference of $14.27 \%$ in peak systole and $12.91 \%$ in diastole relative to maximum 
velocity measured at each cardiac phase.

However, also numerical simulations have their own limitations which resides in model assumptions and simplifications (e.g. as rigid vessel wall) that could alter the flow structures and as a consequence affect the calculated parameter such as WSS [13].

\subsection{Hypothesized role of temperature in atherogene- sis}

A parameter that plays an important role on the biology of vascular cells, is temperature. It is a primary physical variable that regulates the functioning of the living cells. It influences the biology of endothelial cells, in particular:

1. it is associated with an increase of the membrane permeability, which regulates blood plasma transport across the endothelium [144] and the permeation of LDL [145]

2. it affects angiogenesis [146]

3. it regulates the release of Nitric Oxide (NO) by endothelium, an antithrombotic molecule [147].

All these aspects play an important role in the process of atherogenesis.

The group lead by G.Caligiuri, A. Nicoletti and G. Franck (Laboratory for vascular Translational Science (LVTS), INSERM) started investigating the potential link between temperature and vascular disease, a subject never explored before. They postulated that temperature could exert a significant biological effect on endothelial cells with a pro-atherogenic phenotype.

Local temperature on the vessel wall is the consequence of heat exchange between blood flow and the vessel itself, therefore a thermal heterogeneity is expected in areas where the velocity distribution is spatially heterogeneous.

This hypothesis has been confirmed by the heterogeneous temperature distributions obtained in various model of bifurcations (Figure 6.1): 


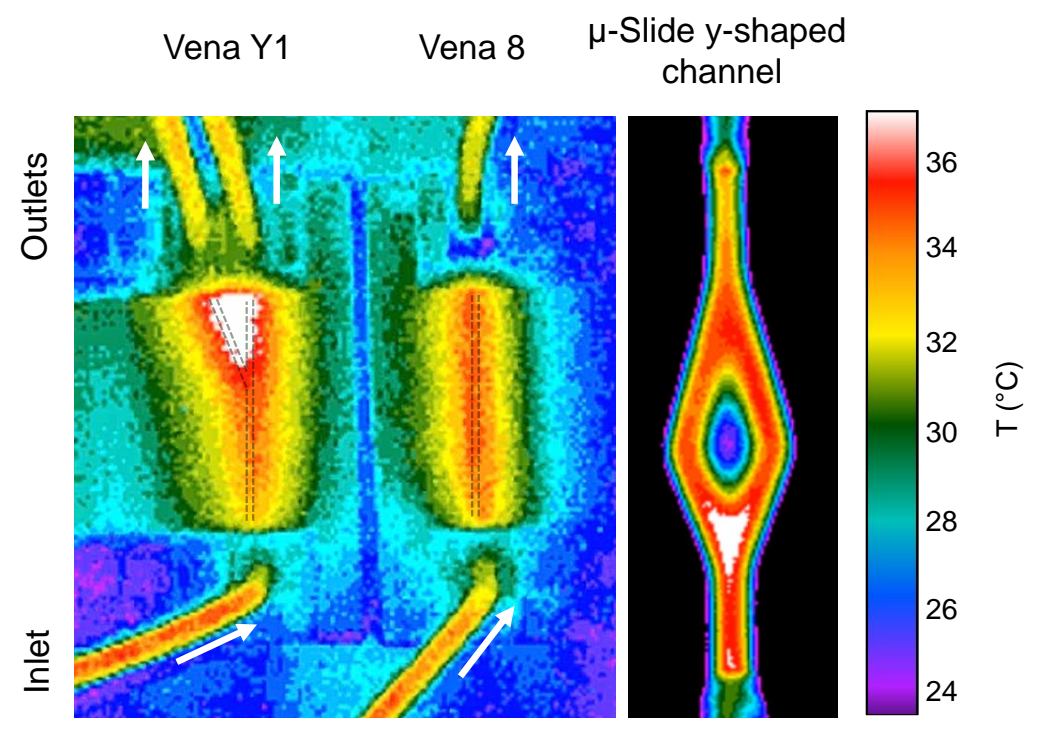

Figure 6.1: Temperature maps at steady state on the surface of a Vena 8 Microfluidic Biochip, a Vena Delta Y1 Microfluidic Biochip and $\mu$-Slide y-shaped channel (Unpublished data, courtesy of LVTS, INSERM).

1. a $\mu$-Slide $y$-shaped channel of a height of $0.4 \mathrm{~mm}$ and width of $3 \mathrm{~mm}$ (Ibidi ${ }^{\circledR}$ Inc., Martinsried, Germany),

2. a Vena Delta Y1 Microfluidic Biochip of a width of $0.08 \mathrm{~mm}$ and a height of $0.12 \mathrm{~mm}$ (Cellix Ltd, Dublin, Irland)

3. a Vena 8 Microfluidic Biochip of a width of $0.8 \mathrm{~mm}$ and a height of $0.08 \mathrm{~mm}$ (Cellix Ltd, Dublin, Ireland)

In particular a $37^{\circ} C$ solution was flowed through them and the system temperature distribution was measured throughout the process using an infra red camera (IRC, Flir Systems, Wilsonville, Oregon, USA). It is clear that in the area close to the bifurcations the temperature is $2^{\circ} C$ higher with respect to the surrounding region, and that the same heterogeneity is not observed in the straight vessel model (Vena 8 in the Figure 6.1).

The main limitation of IRC temperature maps is that the only information that could be retrieved is the surface temperature. Therefore this approach is limited to simple phantoms without a surrounding environment, and it is not applicable in vivo. A great alternative is offered by Magnetic Resonance Thermometry (MRT). 
Magnetic Resonance Thermometry (MRT) is a technique exploiting the temperature dependence of the proton resonance frequency (PRF) [148]. This method is the gold standard to monitor the process of an interventional procedure that relies on a thermal effect. Unfortunately the main limitation of PRF is its inability to provide absolute temperature measurements but only relative ones.

Therefore MRT relies on two acquisitions whose phases are then subtracted to calculate the temperature change due to the intervention. This approach was used in this study, to measure the temperature difference between two states, as it will be shown.

In this chapter a model of the carotid bifurcation, one of the most atheroprone vessels, is used to investigate the role of temperature in the mechanism of atherogenesis by studying the WSS and the temperature distributions. In particular in order to cope with the resolution limitations of flow sensitive MRI and MRT and the modelling assumptions of CFD, EMRI will be used to estimate temperature distribution and WSS.

\subsection{The carotid bifurcation}

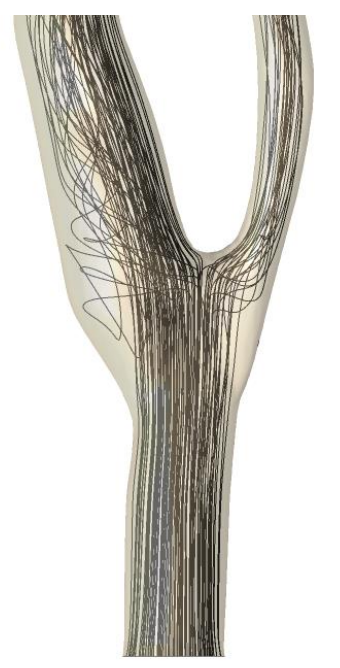

Figure 6.2: Streamlines in a carotid bifurcation.

The carotid bifurcation, one of the most atheroprone vessels in the human vas- 
cular system, is the anatomical region where the common carotid artery (CCA) branches into the internal and the external carotid arteries (ICA and ECA respectively), which supply blood to the intra- and extracranial vessel networks.

This particular geometry causes complex flow structures (Figure 6.2), such as flow separation and recirculation, which lead to a peculiar distribution of shear stresses. In particular the areas located along the outer wall, opposite of the flow divider of the bifurcation, are the ones which are atheroprone [149]. These areas are characterized by low and oscillatory WSS.

In vitro studies in scale models of the human carotid bifurcation comparing flow velocity and wall shear stress with intimal plaque thickness at corresponding locations in carotid bifurcations obtained from cadavers, have highlighted that intimal thickening and atherosclerotic plaques locations, positively correlate with regions of low and oscillatory shear stress, while areas of high shear stress are atheroprotected $[138,150]$.

Preliminary temperature measurements were conducted by the LVTS group with an IRC on a 3D printed scaled model of a rabbit carotid bifurcation in Polylactic Acid (PLA) (Figure 6.3 a) with inner diameter of $7 \mathrm{~mm}$, wall thickness of $1 \mathrm{~mm}$ (printed with a MAKERGEAR ID-M3 (MakerGear LLC, Beachwood, OH, USA)). The ICA and the ECA were then branched into the same outlet, and the resulting model length was $58.6 \mathrm{~mm}$.

In particular water at $37^{\circ} \mathrm{C}$ was flowed through the phantom at room temperature $\left(23^{\circ} \mathrm{C}\right)$ using a continuous pump (mass flow rate $=(2.70 \pm 0.02) \cdot 10^{-3} \mathrm{~kg} \mathrm{~s}^{-1}$ ) and the temperature was measured until the steady state was reached (Figure 6.3 $b$ and $c)$.

The map and the graph show a heterogeneous temperature distribution at the steady state, with a peak of temperature in the sinus of the bifurcation. These observations are again limited to the surface of the model. In order to investigate further these results and assess their reproducibility, a phantom suitable for MRI experiment was realized. The objectives of this study were:

1. Use the MRI to assess the temperature distribution with MRT and the flow 
field with 4D Flow CMR.

2. Use EMRI to improve the resolution of the velocity field obtained with $4 \mathrm{D}$ Flow CMR as well as to retrieve the resulting temperature distribution.

a

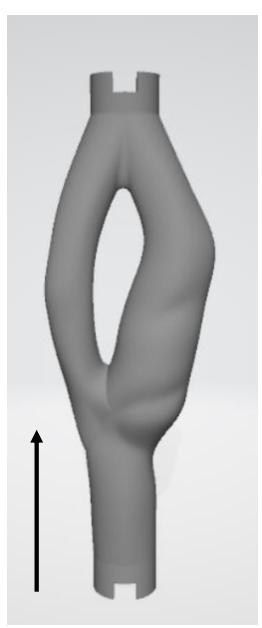

b

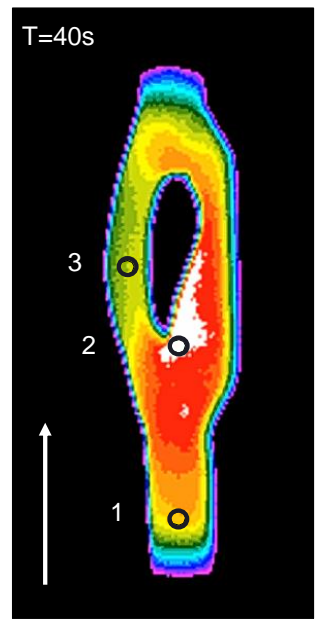

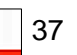
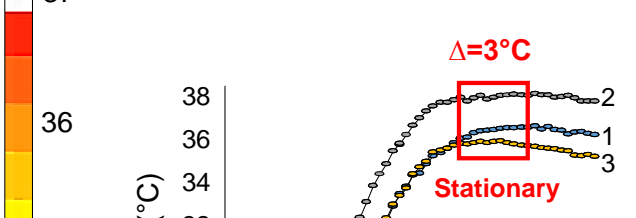

35

34

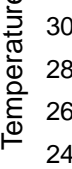

28
26
24
22

22
20

Figure 6.3: a) PLA model of the carotid bifurcation; b)temperature map at the steady state in the carotid bifurcation phantom, measured with IRC; c) Graph showing the temperature change in time of three areas marked in b), in the transitional phase and in the steady state (Unpublished data, courtesy of LVTS, INSERM).

\subsubsection{MRI experimental setup}

In order to perform experiments in MRI a new carotid phantom had to be built. In particular the MRI acquisition had to be performed using a pre-clinical MRI scanner (Bruker 7T Biospec system, Bruker, Ettlingen, Germany) using the Bruker transmit-receive volume coil, which had a $40 \mathrm{~mm}$ inner diameter and $75 \mathrm{~mm}$ outer diameter (Figure 6.4). Therefore the model was realized to be compatible with the coil dimensions.

Firstly a scaled mouse carotid bifurcation phantom (CCA inner diameter 3.32 $m m$, wall thickness $1 \mathrm{~mm}$ ) was designed with a surrounding cylindrical structure (Figure $6.5 \mathrm{a}, \mathrm{b}$ ) with an outer diameter $18.75 \mathrm{~mm}$ and length $30.31 \mathrm{~mm}$ that would host glycerine (Figure $6.5 \mathrm{c}$ ). The phantom was 3D printed with a Formlab Form2 (Formlab, Somerville, Massachusetts, United States). 


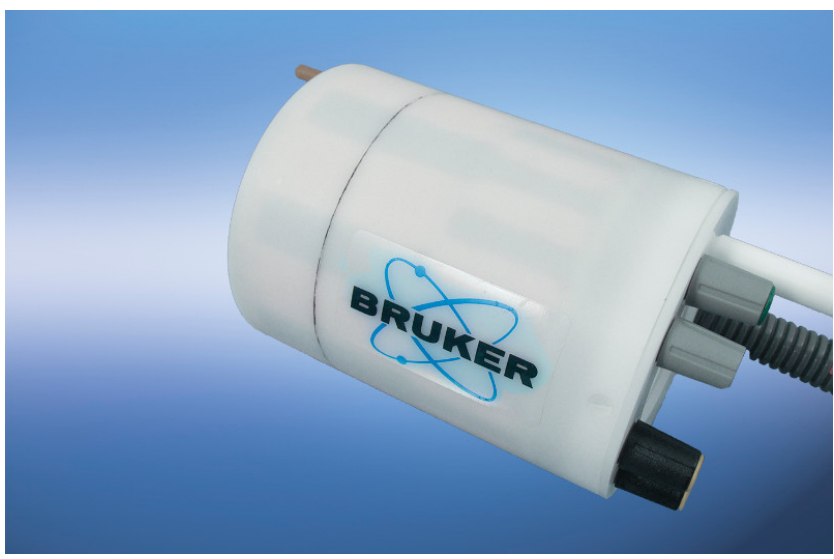

Figure 6.4: Bruker transmit-receive volume coil

The glycerine surrounding the bifurcation lumen enhanced the MRI signal and minimised noise around the wall, the area of main interest. In order to monitor the temperature a probe was fixed in the cylindrical chamber (Figure $6.5 \mathrm{c}$ ).

a

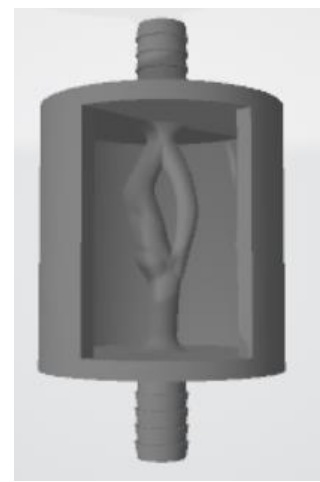

b

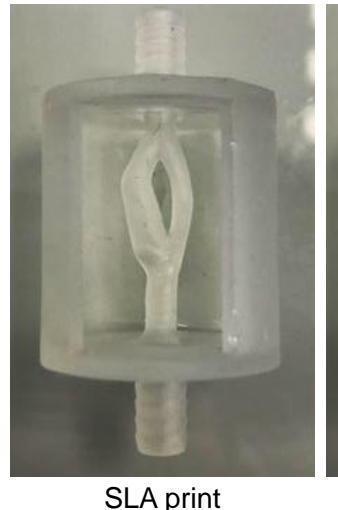

SLA print

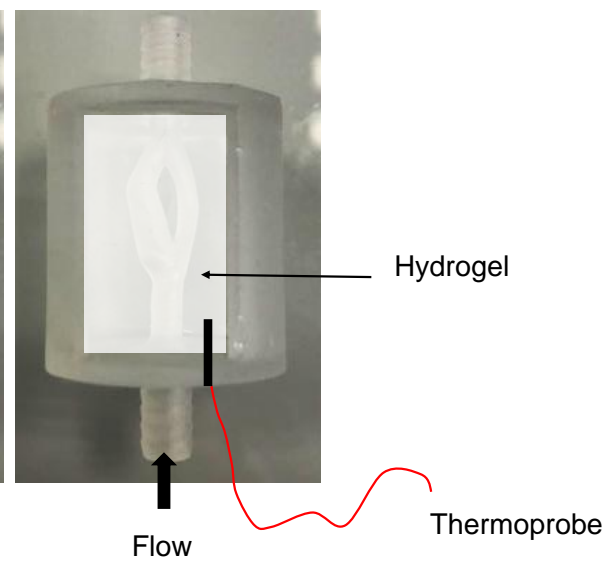

Figure 6.5: a) 3D geometry of the bifurcation phantom b) 3D printed phantom c) experimental setup for MRT: the 3D printed phantom was filled up with an hydrogel to enhance MRI signal and avoid noise on the wall of the bifurcation; moreover in order to monitor the temperature, a temperature probe was inserted.

The phantom was placed in a room at $20^{\circ} \mathrm{C}$ and water at $30^{\circ} \mathrm{C}$ was run through it using a continuous pump (same as in the previous experiment described in section 6.3), until steady state was reached (this was assessed using the temperature probe 
MouseOx® Plus Pulse Oximeter for Rodents, Harvard Apparatus). The phantom was afterwards imaged using an in-house sequence MRT with the following parameters: TR $12 \mathrm{~ms}$, TE $1.91 \mathrm{~ms}$, flip angle $9^{\circ}$, matrix $128 \times 128$, pixel dimension 0.25 $x 0.25 \times 0.533 \mathrm{~mm}^{3}$, number of slices 60 , number of averages 3 .

This approach however showed some flaws. In Figure 6.6 it is clearly visible that the area around the wall of the resin phantom is noisy, due to the fact that the resin does not produce MRI signal due to the very low content of hydrogen. This constitutes a limitation since the region around the vessel wall is the one of interest, therefore a novel approach had to be adopted.

a

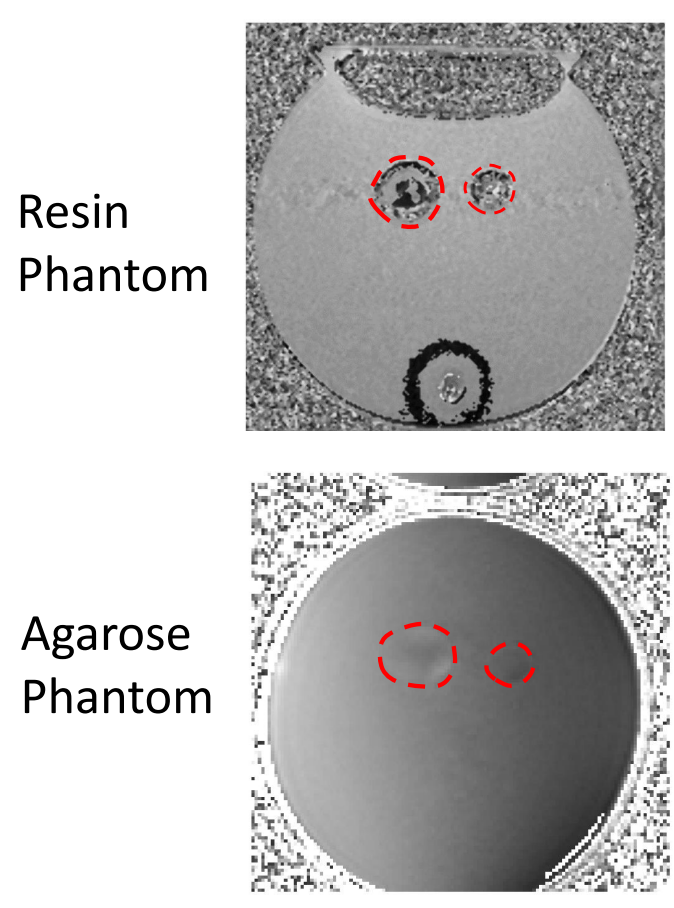

b
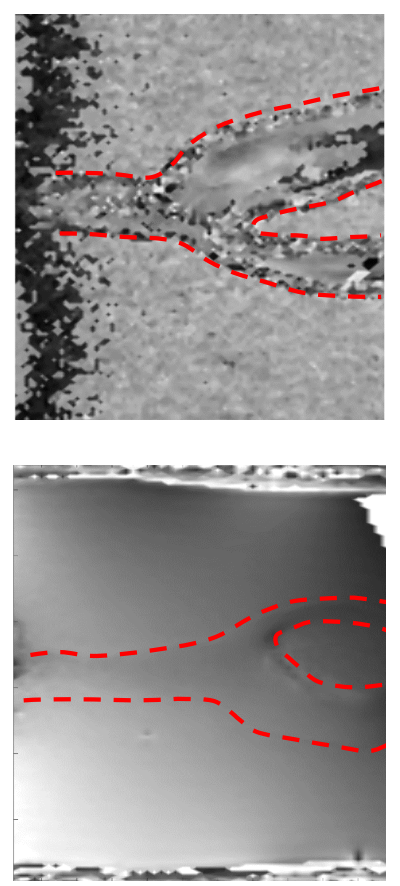

Figure 6.6: Resin phantom and Agarose phantom phase images: transverse (a) and coronal view (b). The red dotted line is showing the vessel wall.

The ideal phantom for the MRT experiment should have the outer box made in a proton rich material, so that the phase signal is good enough to monitor the temperature in the surrounding area. Most importantly, also the wall of the phantom should be made of a similar material, so that information on the wall could be gathered to explore the study hypothesis. 
The PVA lost core approach [1] (the one adopted for the silicon phantom in the validation experiment) was used using agarose gel (4 $g$ of agarose, $100 g$ of water) to manufacture the phantom. The process is shown in Figure 6.7. The bifurcation lumen was 3D printed in PVA (using a MAKERGEAR ID-M3, MakerGear LLC, Beachwood, OH, USA) and covered with acrylic spray paint (PlastiKote, UK), and the outer mould for the cylindrical box was manufactured in resin (using a Peopoly Moai, Hong Kong).

\section{Development of a vascular phantom for MRI thermography}

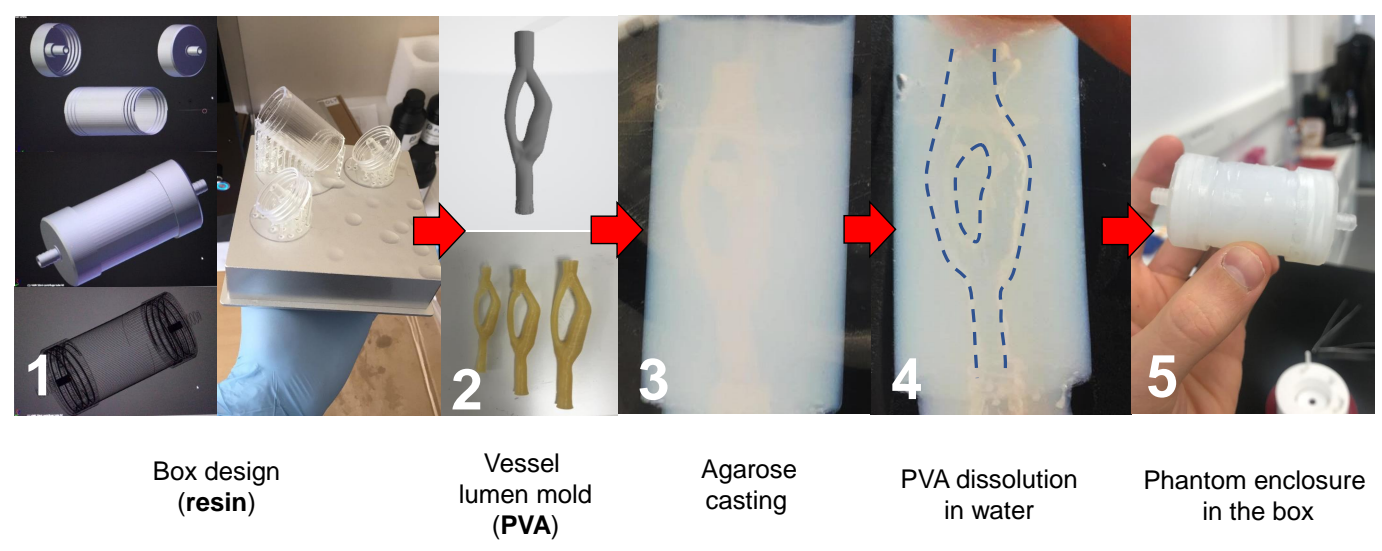

Figure 6.7: Development of vascular phantom for MRT: 1) outer box design and printing in resin; 2) lumen mold printing in PVA; 3) phantom resulting after the casting with agarose gel; 4) phantom obtained after the dissolution of the lumen mould in water (the blue dotted line is showing the vessel wall); 5) phantom enclosed in the resin box.

Afterwards the PVA bifurcation was fixed inside the resin box and the agarose solution was poured in. Once the agarose was solidified the phantom was placed in a water bath to melt the PVA core. In this way a single material phantom was obtained, with the possibility of gathering signal in proximity of the bifurcation wall.

The experiment was repeated using the same protocol as for the PLA phantom. The Figure 6.6 shows the transverse and the coronal sections of the phase of 
the MRT signal. It is clear that the agarose phantom overcomes the problems characterizing the resin phantom as in this case the wall of the vessel is the outer box itself, avoiding noise in proximity of the bifurcation wall.

This manufacturing approach allowed me to perform temperature measurements near the wall of the bifurcation, and to perform 4D Flow CMR, that was used to set up the EMRI model.

\subsubsection{4D Flow CMR}

The phantom was imaged using an in-house 4D Flow CMR sequence: TR 15.49 $m s$, TE $5.1265 \mathrm{~ms}$, flip angle $20^{\circ}$, matrix $128 \times 128$, pixel dimension $0.25 \times 0.25$ $x 1.6 \mathrm{~mm}^{3}$, number of slices 20 , number of phases 1 , number of averages 3 . The velocity encoding was made in three orthogonal directions: head-foot, right-left and anterior-posterior with a $v_{E N C}$ of $0.20 \mathrm{~ms}^{-1}$. The SNR, computed adopting the same methodology illustrated in the previous chapter, was of $30.5 \mathrm{~dB}$.

\subsubsection{EMRI}

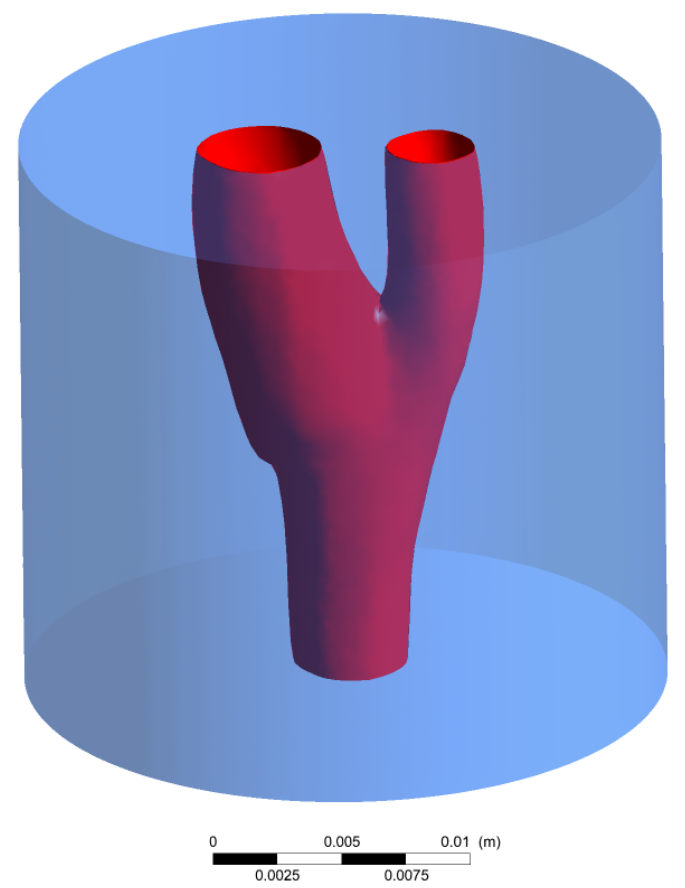

R18.0

Figure 6.8: Geometry of the carotid artery and on the surrounding box. 
The anatomy of the bifurcation was segmented from magnitude images, averaging the three encoding directions, using Simpleware ScanIP (version O-2018.12SP2, Synopsys, CA, USA) and meshed using ICEM CFD (ANSYS Inc, Cannonsburg, PA, USA).

The geometry (Figure 6.8) was meshed using tetrahedral elements and prism elements for the boundary layer (8 layers) near the wall. The mesh (chosen after a sensitivity test, see A.4) had 1015999 nodes and 4139774 elements.

Let us briefly introduce how the heat transfer was modelled. Together with the conservation of momentum and mass, explained in chapter 1.3.1, in the case of heat exchange we need to consider the energy conservation equation in the case of incompressible fluid (equation 6.1):

$$
\rho c_{p}\left[\frac{\partial T}{\partial t}+(v \cdot \nabla) T\right]=k \nabla^{2} T+\phi
$$

where $\mathrm{T}$ is the temperature, $c_{p}$ is the specific heat at constant pressure, $k$ is the thermal conductivity and $\phi$ is a dissipation function that represents the work done against viscous forces, expressed as (Equation 6.2):

$$
\phi=(\tau \cdot \nabla) v=\tau_{i j} \frac{\partial v_{i}}{\partial x_{j}}
$$

where $\tau_{i j}$ is the deviatoric stress tensor.

In the case of an incompressible fluid a state equation does not exist, therefore the energy equation is decoupled from the other two. This means that continuity and NS equations can be solved to find velocity and pressure fields. Afterwards this solution is used to compute the temperature distribution.

The boundary conditions of the bifurcation lumen were the following:

1. inlet: mass flow rate of the pump $\left(2.70 \cdot 10^{-3} \mathrm{~kg} \mathrm{~s}^{-1}\right)$, and water temperature of $30^{\circ} \mathrm{C}$.

2. outlet: opening 
3. wall: no-slip boundary condition;

the boundary conditions of the cylindrical box are:

1. outer wall: fixed temperature of $20^{\circ} \mathrm{C}$.

2. inner wall: conservative interface flux.

The flow was modelled as laminar, with Newtonian and incompressible fluid (water, density and viscosity). The walls were modelled as rigid (with this flow regime the agarose has a rigid behaviour). 4D Flow CMR data were processed using an inhouse code written in Matlab (Matlab, MathWorks, Natick, MA), to retrieve the velocity field in the entire bifurcation domain.

The EMRI model used the additional external force term that made use of the 4D Flow CMR measured velocity field. In this case a scaling of 0.18 was used and the wall function was applied using a $\Delta$ Pix of $0.137 \mathrm{~mm}$ (for simplicity the same ratio pixel to $\Delta$ Pix found in the aorta application was used). The velocity and temperature maps estimated with EMRI were compared with those derived with MRI.

\subsubsection{Results and discussion}

\subsubsection{Velocity and temperature maps}

The velocity maps of a longitudinal and a cross-sectional plane of the carotid phantom, measured with 4D Flow CMR and computed with EMRI are shown in Figure 6.9. The velocities obtained are in the same range $\left(0-0.3 \mathrm{~ms}^{-1}\right)$. EMRI velocity maps are smoother than the 4D Flow CMR maps. Moreover the non-slip boundary condition allowed to correct the velocity values in proximity of the wall.

The temperature maps of a longitudinal and a cross-sectional plane of the agarose box surrounding the lumen of the carotid phantom, measured with MRT and computed with EMRI are shown in Figure 6.10. In both the planes the overall distribution is consistent, even though MRT maps shows a less smooth distribution; a consequence of the lower spatial resolution. 
4D Flow CMRI

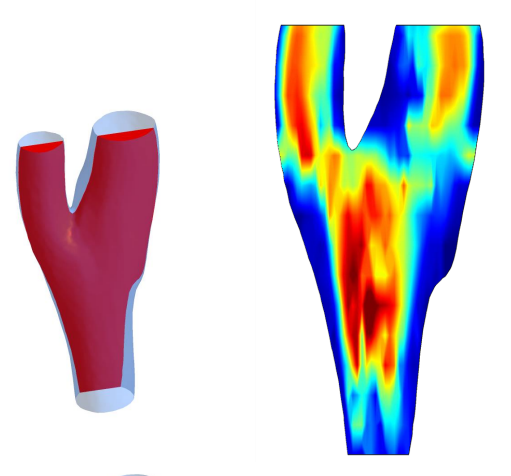

Ho
EMRI

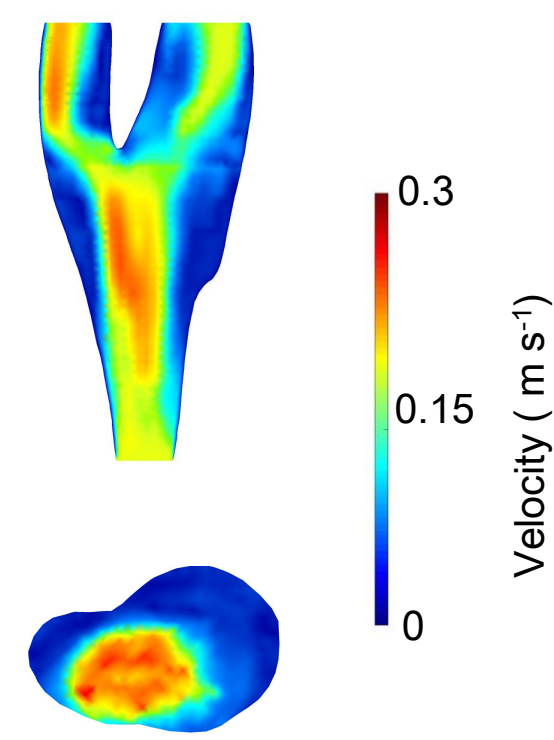

Figure 6.9: Velocity maps of the carotid bifurcation computed with 4D Flow CMR and EMRI. Sagittal cutting plane (top row) and transversal cross section (bottom row). The locations of cutting planes are shown on the left panel.

\subsubsection{WSS distribution and temperature heterogeneity}

The velocity data were used to compute the WSS. In particular the same approach used for the patient specific aorta data was adopted for the 4D Flow CMR data acquired on the carotid phantom, using a $d_{W A L L}$ threshold of half a pixel $(0.125$ $\mathrm{mm}$ ). The WSS maps of the carotid phantom, computed with 4D Flow CMR and EMRI are shown in Figure 6.11.

In order to estimate the temperature on the 'carotid wall', an offset surface was created by moving the lumen points normally by $1 \mathrm{~mm}$ from the initial surface in the outward direction in the outer box. In Figure 6.12, the temperature maps computed by EMRI on this surface are presented.

Both sides of the carotid phantom show a temperature increase at the bifurcation and in particular where the CCA is branching. These results are qualitatively consistent with the IRC observations. In order to understand the origin of the temperature increase observed, the Nusselt number, expressing the ratio of convective 
MRT
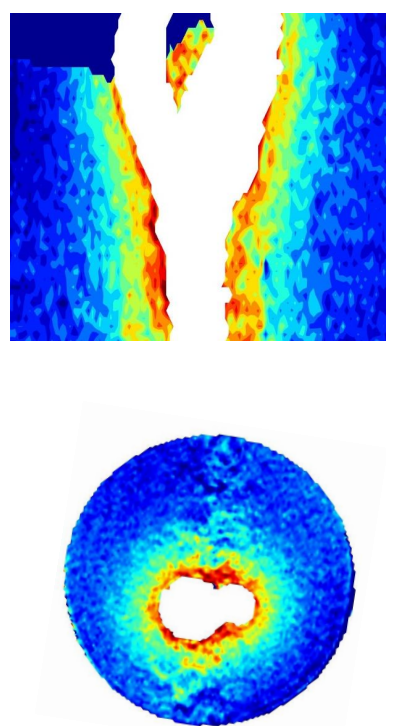

EMRI
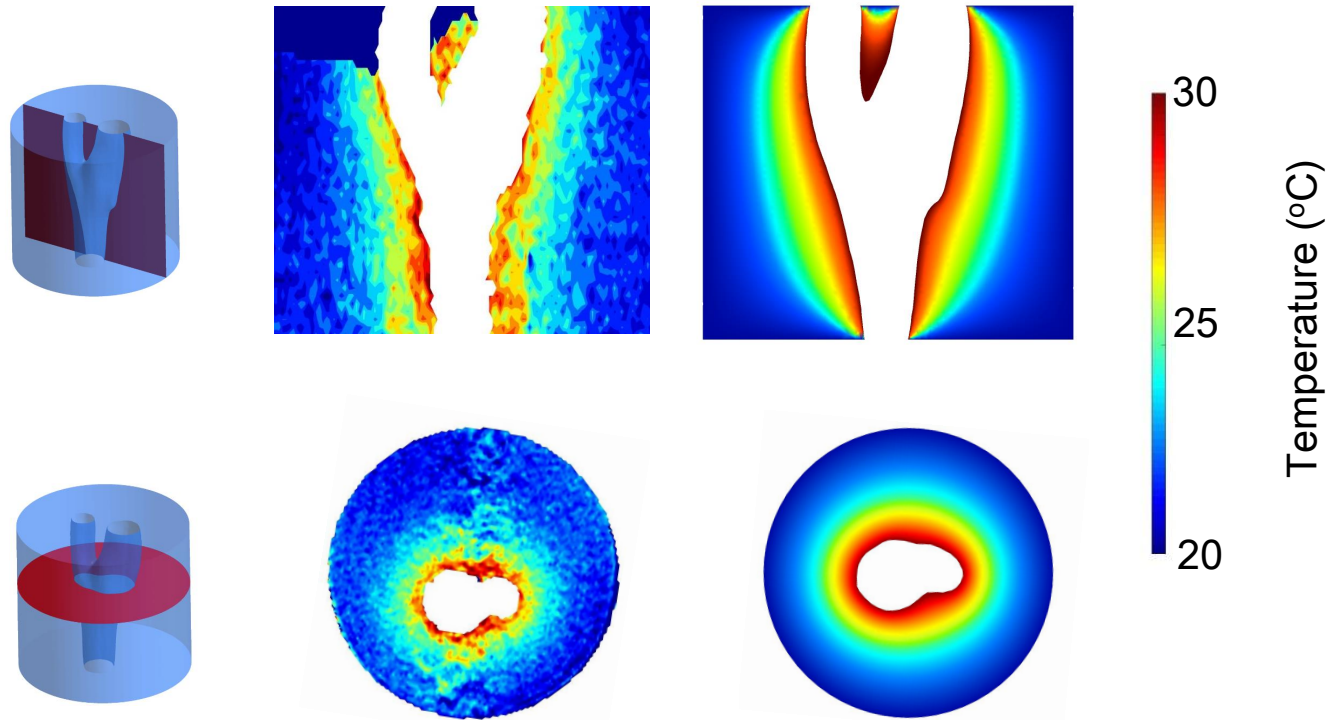

Figure 6.10: Temperature maps of the box surrounding the carotid bifurcation computed with MRT and EMRI. Sagittal cutting plane (top row) and transversal cross section (bottom row). The locations of the planes are shown on the left panel.

to conductive heat transfer, was computed as follow (Equation 6.3):

$$
N u=\frac{h x}{k}
$$

where $\mathrm{h}$ is the convective heat transfer coefficient of the flow, $\mathrm{x}$ is the distance from the boundary (in this case $1 \mathrm{~mm}$ ), and $\mathrm{k}$ is the thermal conductivity of the fluid ( $0.61450 \mathrm{~W} \mathrm{~K}^{-1}$ for water at $303.15 \mathrm{~K}$ ). The contour plot of the Nusselt number for the carotid bifurcation are shown in figure 6.13:

The average Nusselt number is 42.85 , which is an indicator of active convection. The Nusselt number distribution shows values in the same range in the CCA while in the sinus the distribution is more heterogeneous. This discrepancy is due to the difference in the velocity patterns and in particular to the fact that disturbances occur at the bifurcation joint. The flow paths are shown in Figure 6.14 overlaid with the temperature distribution on the vessel wall.

This analysis shows that the temperature distribution correlates in a satisfactory 
4D Flow CMRI
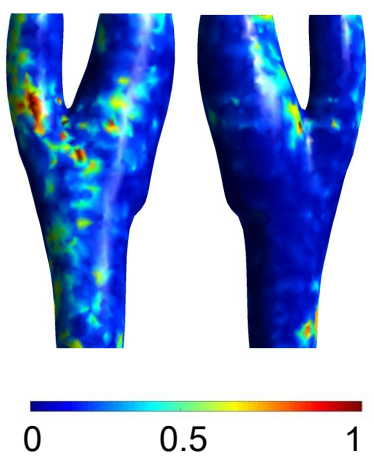

WSS $(\mathrm{Pa})$
EMRI

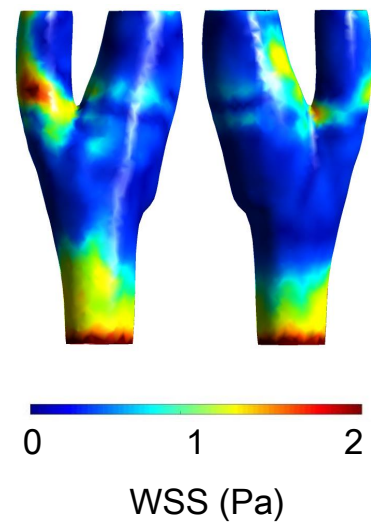

Figure 6.11: WSS maps of the carotid bifurcation phantom, computed with 4D Flow CMR (on the left )and EMRI (on the right). The two sides of the vessel are shown.
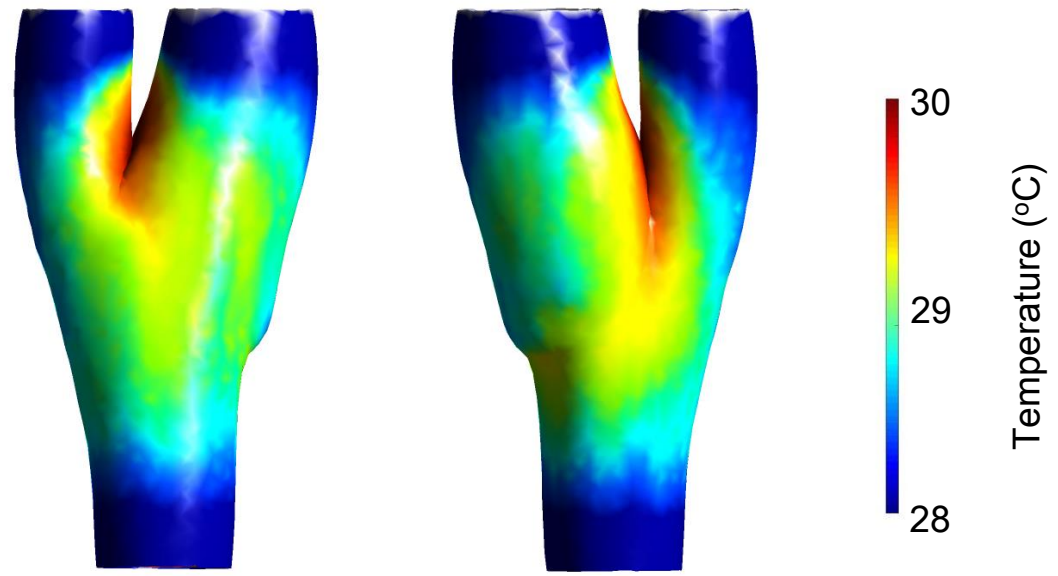

Figure 6.12: EMRI temperature maps computed on an surface at $1 \mathrm{~mm}$ offset from the lumen of the bifurcation model.

way with the flow patterns, which in this case are retrieved with the EMRI approach. In the next section, preliminary in-vivo results are shown. 

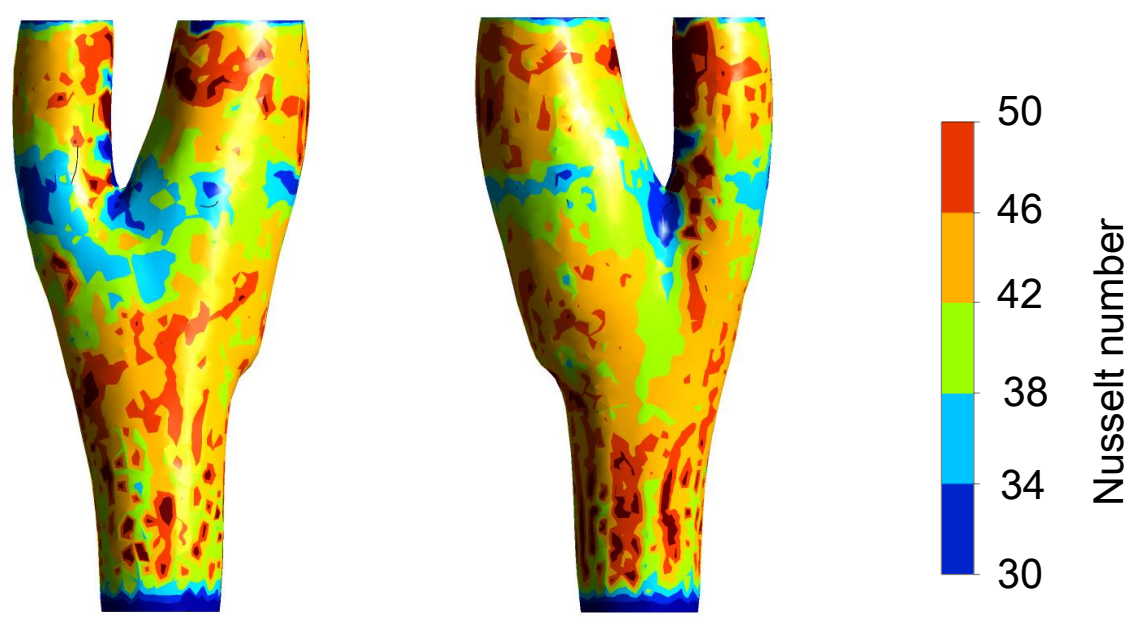

Figure 6.13: Contour plot of the Nusselt number on the two sides of the carotid bifurcation wall.

\subsection{Translation to animal study: preliminary results}

An ApoE - / - mouse was anaesthetized with $2 \%$ isoflurane inhaled anesthetic. The mouse was placed on an heated bed and the ECG and the respiration were monitored during the MRI acquisition (Figure 6.15). The same MRT sequence used for the phantom was performed on the mouse with ECG and respiration gating.

Figure 6.16 shows the magnitude (3D volume visualization) on the left side of the picture and the MRT phase maps on the right hand side. Shimming was performed in the area around the left subclavian artery bifurcation (shown by the black dotted line in Figure 6.16), in order to ensure magnetic field homogeneity in this area. The choice of this bifurcation came from the fact that the size of the vessel allowed an higher SNR.

An increase in the phase was observed in the shimmed area at the bifurcation location, as the Figure 6.16 indicates. However as previously mentioned, the MRT sequence could provide only relative phase change, therefore absolute temperature estimation was not possible. 

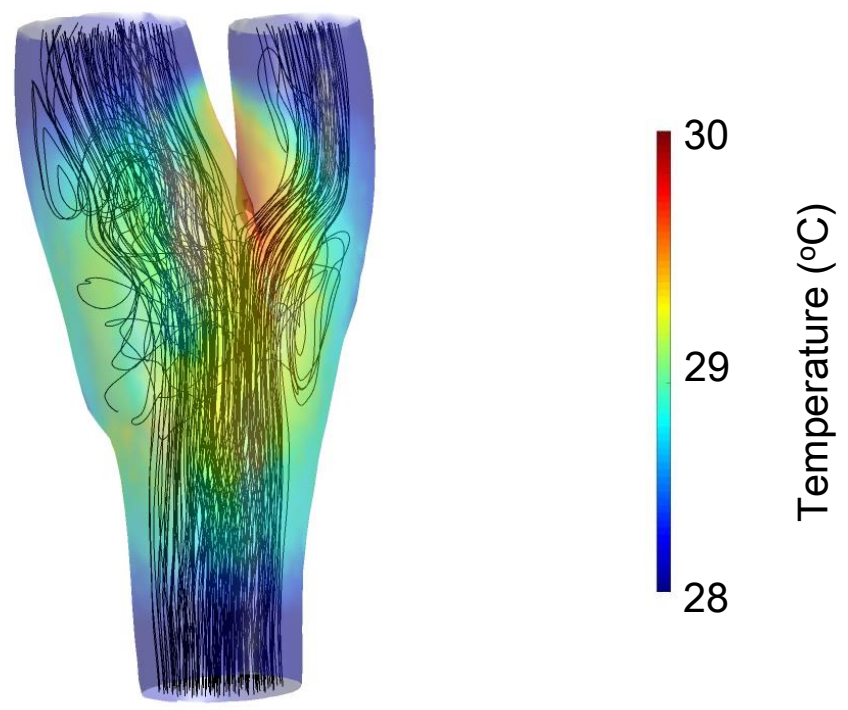

Figure 6.14: Streamlines in the carotid bifurcation with velocity map overlaid.

a)

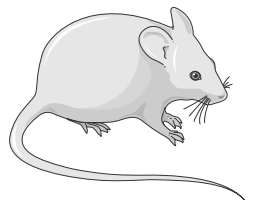

b)

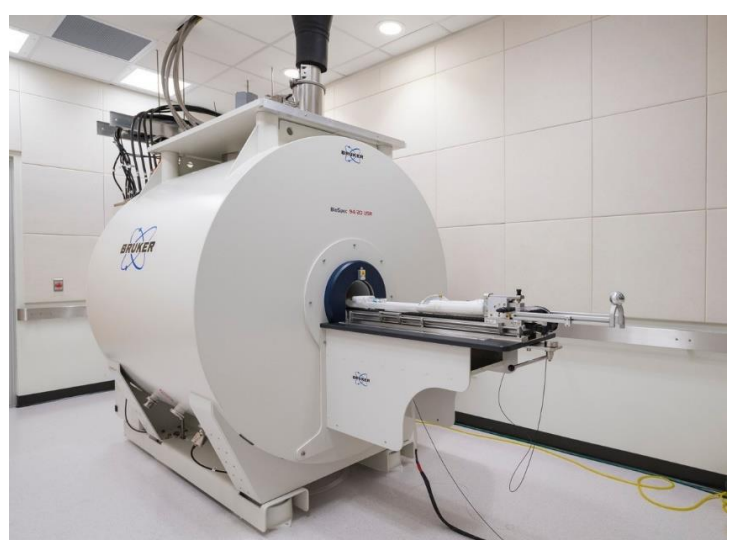

Figure 6.15: a) ApoE $-/-$ mouse prepared for the scanning. b) Bruker MRI scanner used for the acquisition.

\subsection{Limitations}

The limitation of this study are related to the uncertain role of temperature in atherogenesis rather than to the EMRI methodology. 
Magnitude

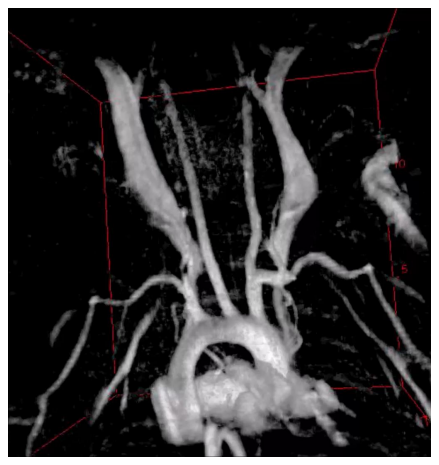

MRT Phase

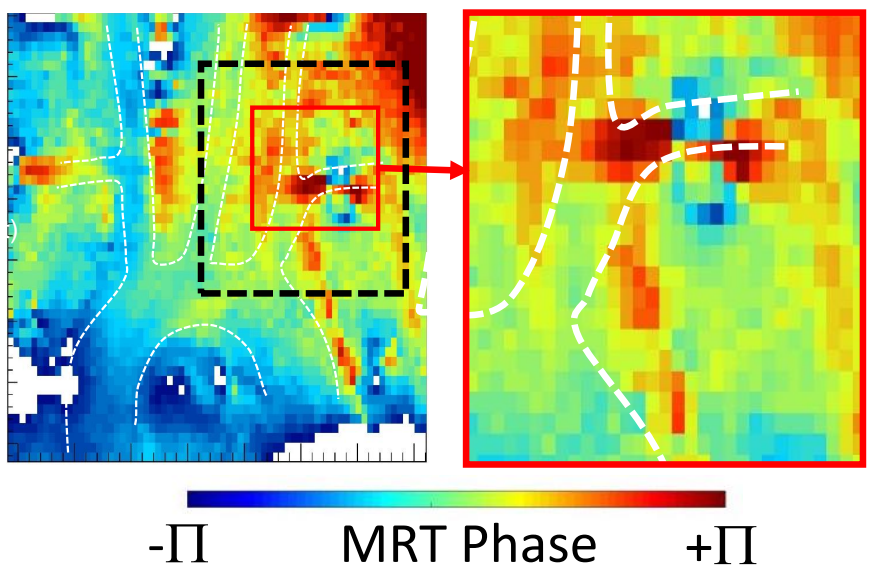

Figure 6.16: Magnitude (3D volume visualization) and the MRT phase maps. The black dotted line in the MRT maps shows the shimmed area. The left subclavian artery bifurcation MRT map is magnified on the right side of the picture.

The main limitation of this study is constituted by the fact that only one carotid model was studied, subject to a non physiological flow and temperature. Therefore the next step would be to perform the same experiment with pulsatile flow and with physiological temperature boundary conditions, whose assessment still constitutes a big challenge.

An alternative strategy to MRT is constituted by spectral magnetic resonance imaging (SMRI) making use of the methylene group resonance frequency in combination with a hydrogen based chemical shift to measure absolute temperature. The methylene group, an ubiquitous and a highly represented molecular group in living tissues, can be used as temperature independent reference, and carries a resonance frequency shift which is easily recordable and almost twice as big as the one of the hydrogen. This would allow an absolute temperature estimation and validate the hypothesis of a role of temperature on the atherogenesis.

In this unexplored field, EMRI could constitute a novel tool to better understand fundamental mechanisms behind atherogenesis, providing access to high resolution data informed by the measured one and replicating more accurately the experimental conditions. 


\section{Chapter 7}

\section{Conclusions and future work}

Blood flow in the main part of cardiovascular system is a fundamental parameter to perform non-invasive diagnosis of cardiovascular diseases. However estimates of velocity by means of 4D Flow CMR are highly affected by noise, poor spatial and temporal resolution, which are inevitable due to limited scan time.

In order to cope with these problems, an approach called Enhanced Magnetic Resonance Imaging (EMRI) has been proposed in which 4D Flow CMR and CFD are synergistically coupled.

This approach has proven to give access to high spatial resolution and fluid dynamically sensible results, which could lead to a significant improvement in the accuracy of cardiovascular biomarkers estimates. This method was implemented in a commercial and widely adopted CFD software, in order to demonstrate ease of implementation. EMRI was tested firstly on a numerical experiment of known analytical solution, to examine its feasibility and analyse the sensitivity of the input parameters on the results. Results demonstrated that even in presence of noise in the experimental data EMRI reproduces velocity profiles within $2.5 \%$ error.

In order to ensure the feasibility of EMRI with 4D Flow CMR data, a 2D portion of the descending aorta was analysed, comparing the velocity distribution obtained.

To validate the suggested method, a $\mathrm{U}$ bend test rig was manufactured and connected to a blood pump. To our knowledge this study constitutes the first attempt to comprehensively compare 4D Flow CMR and CFD flow field, against PIV. In 
particular the system was imaged with a 4D Flow CMR sequence and the measured flow patterns were compared with the ones of standard CFD and EMRI, using high resolution in-plane PIV as the reference velocity field. EMRI flow patterns showed a better qualitative and quantitative agreement with the ones measured with PIV, than flow patterns obtained by means of CFD alone. Moreover EMRI enable estimation of fluid-dynamically sensible flow quantities, thanks to the constraints required to fulfill Navier-Stokes equations, and achieve high resolution in the boundary layer region, correcting the errors of MRI in the region near the walls.

Afterwards the validated method was then tested on a healthy patient specific aorta. A more complex aortic flow pattern was reproduced using EMRI, compared to standard CFD. At the same time, EMRI gave smooth and fluid-mechanicallysound patterns of blood flow velocity (e.g. zero velocity on the wall, conservation of mass and momentum, etc.). Thus, the limit in the spatial resolution of the 4D Flow CMR could be compensated for by EMRI, while the experimental error was corrected by the fluid mechanical 'filter' (by enforcing the obedience to NavierStokes equations), with the possibility of deriving additional variables such as WSS.

Stresses on the vessel walls have been proven to be directly linked with cardiovascular diseases such as atherosclerosis [59] and aneurysms [151], thus EMRI could allow better understanding of these conditions from a fluid dynamic perspective.

Furthermore, this application demonstrated the ability of EMRI to give reliable results even when considering a small portion of the domain. This last feature could be a good asset for clinical translation, since it would allow EMRI to be run in the area of interest only, thus saving lots of computational time and resources.

The enhanced velocity field accessible via EMRI triggered the idea that the suggested methodology could play a major role in estimating flow-dependent parameters, and in particular temperature distribution in vessels.

A carotid phantom was realized and then studied using 4D Flow CMR, CFD and EMRI. Moreover temperature distribution was imaged using MRT. This application of EMRI aimed at investigating the existence of a relationship between 
temperature heterogeneity and WSS, using EMRI to compensate for the resolution limitation and noise of MRI. Also in this context EMRI allowed a more realistic description of flow and flow-dependent quantities, leading to a more accurate WSS and temperature distribution. These findings confirmed the potential of this methodology also in fundamental research, in particular in the context of atherogenesis.

Three major items in the next phase of EMRI development would be:

- to extend the method for transient analysis;

- include the pressure correction term in the EMRI framework in order to apply this methodology for non-invasive dynamic pressure assessment;

- an application to a pathological scenario, e.g aortic coarctation.

The transient implementation of EMRI requires the careful selection of adequate timing and latency of the external force application, as demonstrated in the numerical example. 4D Flow CMR velocity maps are the result of an average over the acquisition time step rather than being instantaneous. Moreover, if the temporal resolution of the EMRI is higher than the 4D Flow CMR, an effective approach to implement the force in the time step where the 4D Flow CMR data are not available will need to be identified and developed.

The application of EMRI to a pathological condition could be vital to obtain better insights into the fluid dynamics characteristics of the disease studied and understand its evolution and consider novel approach to the treatments.

The non invasive assessment of pressure would constitute a main advantage. Therefore the first step would be to implement the pressure correction term in the EMRI framework and afterwards to perform a validation against gold standard methods for pressure assessment, such as catheter measurements. As mentioned aortic coarctation could be a good candidate as the pressure gradient across the aortic stenosis, that characterizes this disease, is the main parameter used to assess the disease severity and the outcome of the intervention.

In clinical practice X-rays/echocardiography are used to assess the morphology of the stenosis, and a measure of the peak systolic pressure gradient is performed 
invasively with cardiac catheterisation. An assessment of the stenosis is performed after the treatment (usually by balloon angioplasty with or without stent implantation) and transthoracic doppler ultrasound and chest X-rays/echocardiography are performed to assess the residual gradient and the aortic morphology, respectively. Despite the disadvantages of pressure assessment by the catheterisation technique (such as invasiveness of the procedure, the use of ionizing radiation, and costs), it is still considered the standard decision-making tool for diagnosis of aortic coarctation, and treatment evaluation.

Non invasive tools for assessing the pressure gradient are still susceptible to errors. Transthoracic Doppler ultrasound overestimates pressure gradients, while 2D PC MRI tends to underestimate pressure gradients [152].

A non invasive alternative to the aforementioned approaches could be EMRI, with the advantage of giving detailed informations about flow field. This application would require first a validation of the pressure map estimated by EMRI in order to then translate the approach to the study of patient specific scenarios.

Finally the relationship between flow pattern and temperature distribution in atheroprone vessels requires further investigation. In particular it could be extended to the physiological scenario, evaluating the magnitude and impact of temperature on atheroprone areas in vivo in a pre clinical setting as a first step. In these cases a careful consideration of the boundary conditions is needed especially for the temperature.

It was shown that EMRI features overcome at the same time the experimental limits of MRI and the modelling simplifications of CFD with the possibility of deriving complex patterns observed experimentally while increasing the resolution and correcting for imaging errors.

This methods has the potential to become a valid tool to be used to understand the onset of cardiovascular diseases in research and to be used in clinics as a powerful diagnostic tool. A wide field of investigation is the role of the flow patterns in cardiovascular diseases, already elucidated by several studies [153, 154], but that still needs to be better comprehended. Therefore, this method shows the potential to 
evolve into an important diagnostic tool on one side, allowing the definition of noninvasive biomarkers to follow-up disease progression or treatments outcome, and on the other side to assist scientists in the understanding of fundamental research problems related to flow. 


\section{Appendix A}

\section{Grid independence tests}

\section{A.1 2D pipe}

The 2D pipe was meshed using 4 different tetrahedral unstructured meshes whose features are reported in Table A.1

Table A.1: Characteristics of the different meshes of the 2D pipe

\begin{tabular}{c|c|c|c|c}
\hline \hline Mesh $n^{o}$ & $\mathbf{1}$ & $\mathbf{2}$ & $\mathbf{3}$ & $\mathbf{4}$ \\
\hline$n^{o}$ of elements & 1125 & 4500 & 18000 & 71281 \\
$n^{o}$ of nodes & 2432 & 9362 & 36722 & 144000 \\
$n^{o}$ of boundary layers & 3 & 5 & 10 & 20 \\
\hline \hline
\end{tabular}

The velocity profile along a section of the $2 \mathrm{D}$ pipe $(\mathrm{y}=0.025 \mathrm{~m}$ from the inlet, FigureA.1 a) ) was computed to assess the convergence and choose the mesh (FigureA.1 b)).

a)

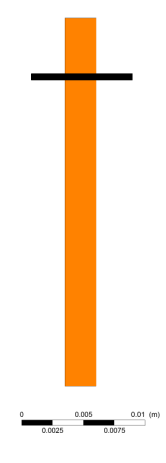

b)

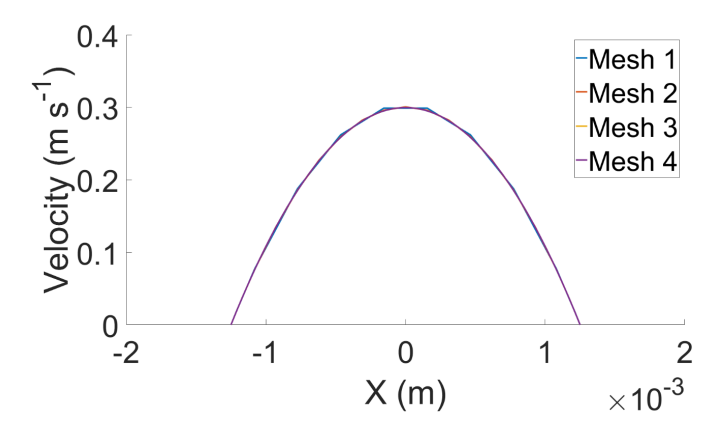

Figure A.1: (a) section where the velocity profiles where computed; (b) velocity profiles computed with the different meshes. 
The velocity field reaches convergence with the mesh $n^{o} 2$, and can be easily stated by looking at the trend of the plot of the number of element vs the peak velocity on the chosen profile (FigureA.2). The discrepancy of the peak velocity computed with mesh $n^{o} 2$ and $n^{o} 1$ is $5.4 \cdot 10^{-5} \%$. However, because of the objective of the study conducted with the $2 \mathrm{D}$ pipe, the most refined mesh was chosen.

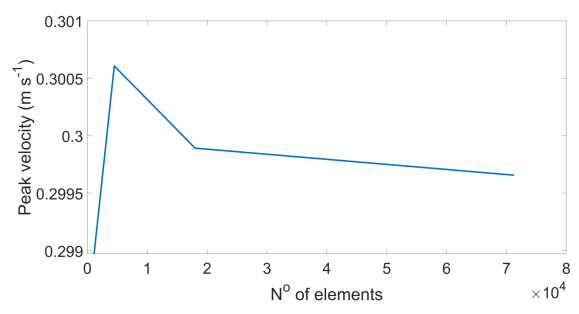

Figure A.2: Plot of the peak velocity computed on the selected line and the number of elements of the meshes.

\section{A.2 Aorta}

The aorta was meshed using 4 different tetrahedral unstructured meshes with the characteristics reported in Table A.2. The velocity profile was computed along a section of the aorta (after the aortic arc, Figure A.3 a) ) to assess the convergence and choose the mesh (FigureA.3 b)).

Table A.2: Characteristics of the different meshes of the aorta.

\begin{tabular}{c|c|c|c|c}
\hline \hline Mesh $n^{o}$ & $\mathbf{1}$ & $\mathbf{2}$ & $\mathbf{3}$ & $\mathbf{4}$ \\
\hline$n^{o}$ of elements & 238498 & 460783 & 1332929 & 3384085 \\
$n^{o}$ of nodes & 82642 & 152961 & 433765 & 953431 \\
$n^{o}$ of boundary layers & 3 & 5 & 10 & 20 \\
\hline \hline
\end{tabular}

Taking into consideration the trend of the plot of the number of element vs the peak velocity on the chosen profile (FigureA.4), it is observable that the velocity field reaches convergence with the mesh $n^{\circ} 3$. The discrepancy of the peak velocity computed with mesh $n^{o} 3$ and $n^{o} 4$ is $1.04 \%$.

\section{A.3 U bend}

4 different meshes of the $\mathrm{U}$ bend were adopted to perform the grid indepence test. The meshes characteristics are shown in Table A.3 
a)

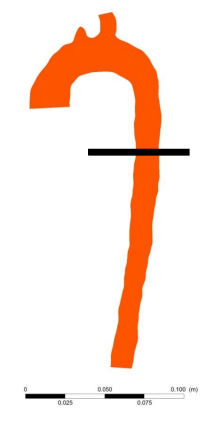

b)

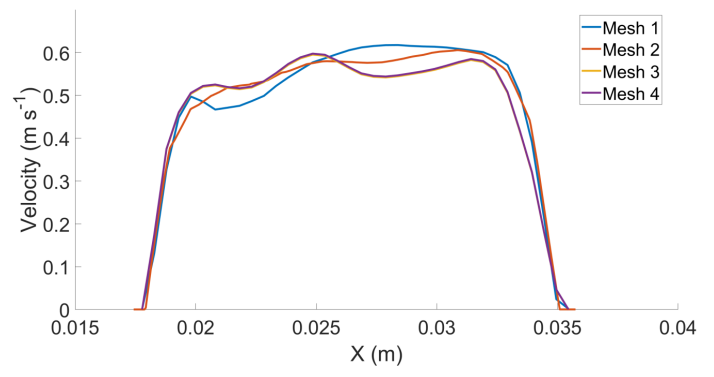

Figure A.3: (a) section where the velocity profiles where computed; (b) velocity profiles computed with the different meshes.

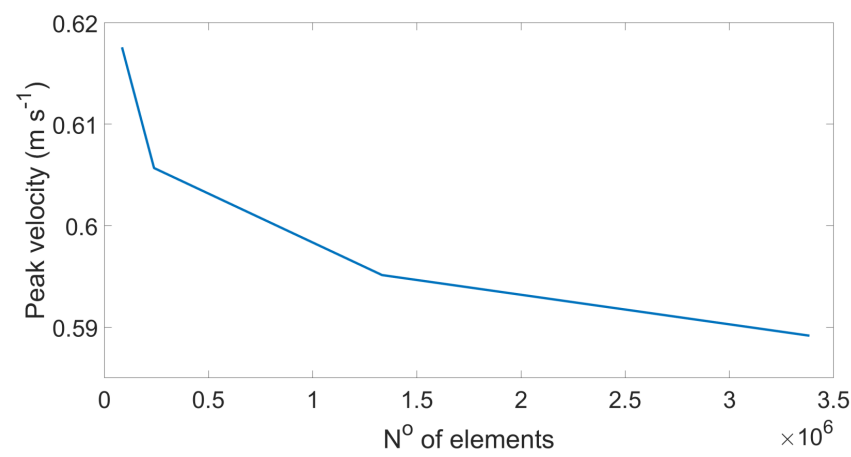

Figure A.4: Plot of the peak velocity computed on the selected line and the number of elements of the meshes.

Table A.3: Characteristics of the different meshes of the U bend.

\begin{tabular}{c|c|c|c|c}
\hline \hline Mesh $n^{o}$ & $\mathbf{1}$ & $\mathbf{2}$ & $\mathbf{3}$ & $\mathbf{4}$ \\
\hline$n^{o}$ of elements & 697399 & 1400330 & 1697646 & 3053481 \\
$n^{o}$ of nodes & 280248 & 518617 & 727498 & 1303490 \\
$n^{o}$ of boundary layers & 8 & 10 & 10 & 10 \\
\hline \hline
\end{tabular}

The velocity profile of the section shown in Figure A.5 a, was computed with all the different meshes to assess the convergence (FigureA.5 b).

The convergence of the velocity field solutions is easy to visualize by plotting the number of element vs the peak velocity on the selected line (FigureA.6). The discrepancy of the peak velocity computed with mesh $n^{\circ} 3$ and $n^{\circ} 4$ is $0.8 \%$. Therefore the mesh $n^{\circ} 3$ was selected. 
a)

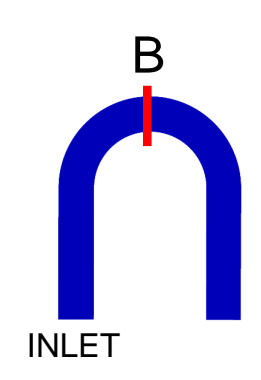

b)

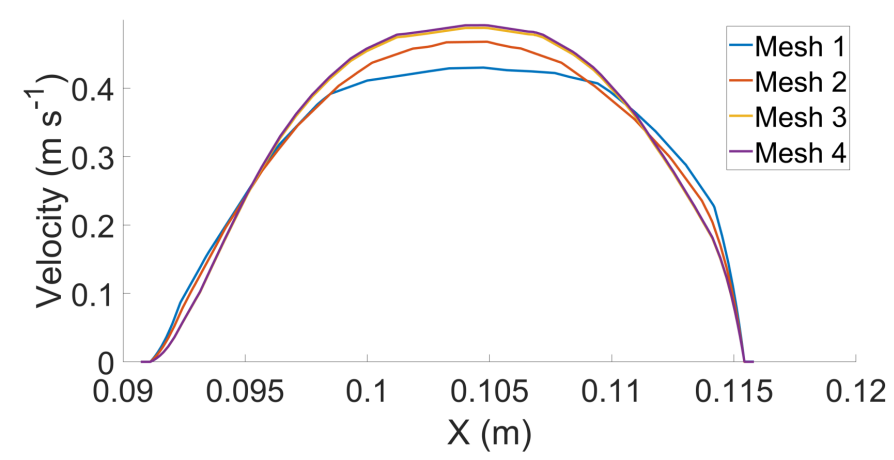

Figure A.5: (a) section where the velocity profile was computed; (b) velocity profiles computed with the different meshes

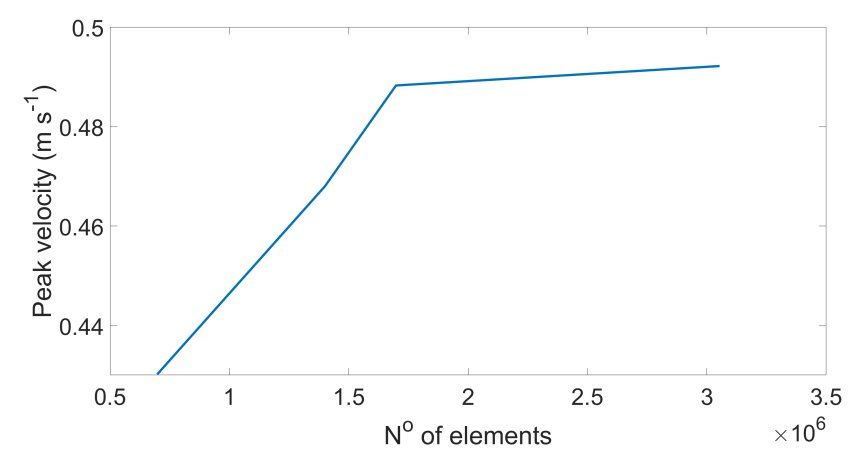

Figure A.6: Plot of the average velocity computed on the selected line and the number of elements of the meshes.

\section{A.4 EMRI model of carotid bifurcation}

The MRI retrieved bifurcation geometry was meshed using 4 different tetrahedral unstructured meshes with boundary layers mad of prisms. The characteristics of the meshes are reported in Table A.4

The velocity and temperature profile (Figure A.7 b) and c) respectively) was computed along a line crossing the geometry (Figure A.7 a) ) to assess the convergence and choose the mesh.

It is easy to see that the temperature and velocity solutions reach convergence 
Table A.4: Characteristics of the different meshes of the agarose model of the carotid bifurcation.

\begin{tabular}{c|c|c|c|c}
\hline \hline Mesh $n^{o}$ & $\mathbf{1}$ & $\mathbf{2}$ & $\mathbf{3}$ & $\mathbf{4}$ \\
\hline$n^{o}$ of elements & 1263453 & 2910800 & 4139774 & 5813096 \\
$n^{o}$ of nodes & 235057 & 541416 & 1015999 & 1092167 \\
$n^{o}$ of boundary layers & 3 & 5 & 8 & 10 \\
\hline \hline
\end{tabular}

a)
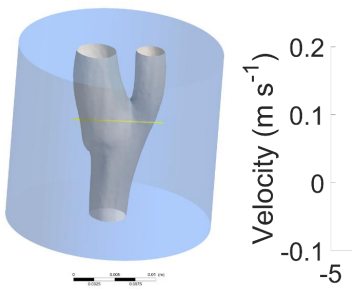

b)

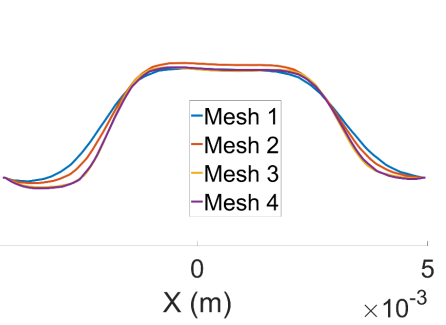

c)

Figure A.7: (a) section where the temperature and velocity profiles where computed; (b) velocity and (c) temperature profiles computed with the different meshes

a)

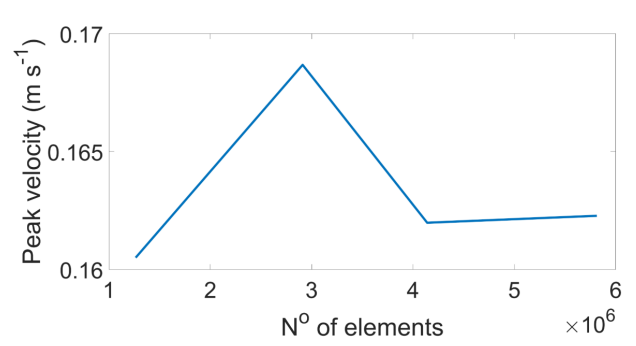

b)

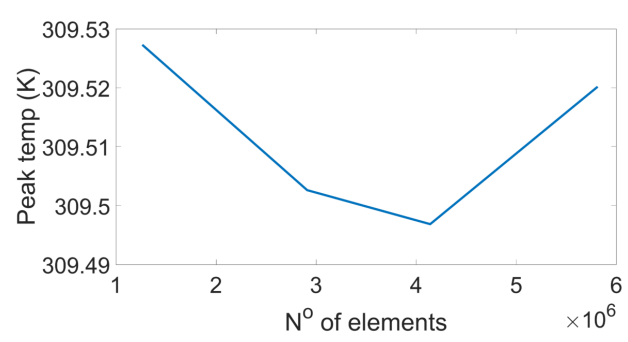

Figure A.8: Plot of the peak velocity (a) and peak temperature (b) computed on the selected line and the number of elements of the meshes.

with the mesh $n^{o} 3$. This is easier to visualize by plotting the number of element vs the peak velocity (Figure A.8 a)) and temperature (Figure A.8 b) on the selected line. The discrepancy of the peak velocity and peak temperature computed with mesh $n^{\circ} 3$ and $n^{\circ} 4$ is respectively $0.1815 \%$ and $0.0075 \%$. 


\section{Bibliography}

[1] Giacomo Annio, Gaia Franzetti, Mirko Bonfanti, Antonio Gallarello, Andrea Palombi, Elena De Momi, Shervanthi Homer-Vanniasinkam, Helge Wurdemann, Victor Tsang, Vanessa Diaz-Zuccarini, Ryo Torii, Stavroula Balabani, and Gaetano Burriesci. Low Cost Fabrication of PVA Based Personalized Vascular Phantoms for in Vitro Haemodynamic Studies: Three Applications. Journal of Engineering and Science in Medical Diagnostics and Therapy, 3(3):1-8, 2019.

[2] Antonio Gallarello, Andrea Palombi, Giacomo Annio, Shervanthi HomerVanniasinkam, Elena De Momi, Gabriele Maritati, Ryo Torii, Gaetano Burriesci, and Helge A. Wurdemann. Patient-Specific Aortic Phantom With Tunable Compliance. Journal of Engineering and Science in Medical Diagnostics and Therapy, 2(4):1-12, 2019.

[3] Giacomo Annio, Ryo Torii, Ben Ariff, Declan P. O’Regan, Vivek Muthurangu, Andrea Ducci, Victor Tsang, and Gaetano Burriesci. Enhancing Magnetic Resonance Imaging With Computational Fluid Dynamics. Journal of Engineering and Science in Medical Diagnostics and Therapy, 2(4):1-11, 2019.

[4] Thekla H. Oechtering, Alex Frydrychowicz, and Hans Hinrich Sievers. Malrotated sinus vortices in straight graft valve-sparing aortic root treatment: A matter of concern? Journal of Thoracic and Cardiovascular Surgery, 154(3):794-797, 2017. 
[5] S. Mendis, P. Puska, and B. Norrving. Global atlas on cardiovascular disease prevention and control. World Health Organization, pages 2-14, 2011.

[6] Zoran Stankovic, Bradley D Allen, Julio Garcia, Kelly B Jarvis, and Michael Markl. 4D flow imaging with MRI. Cardiovascular diagnosis and therapy, 4(2):173-192, 2014.

[7] Sharon and others Netter, Frank Henry and Colacino. Atlas of Human Anatomy, 1989.

[8] Timothy J Carr, James C and Carroll. Magnetic resonance angiography: principles and applications. Springer Science \\& Business Media, 2011.

[9] Aurélien F. Stalder, M. F. Russe, A. Frydrychowicz, J. Bock, J. Hennig, and M. Markl. Quantitative 2D and 3D phase contrast MRI: Optimized analysis of blood flow and vessel wall parameters. Magnetic Resonance in Medicine, 60(5):1218-1231, 2008.

[10] Juan R Cebral, Christopher M Putman, Marcus T Alley, Thomas Hope, Fernando Calamante, Inova Fairfax Hospital, and Falls Church. Hemodynamics in Normal Cerebral Arteries: Qualitative Comparison of 4D PhaseContrast Magnetic Resonance and Image-Based Computational Fluid Dynamics. Journal of engineering mathematics, 64(4):367-378, 2009.

[11] Loic Boussel, Vitaliy Rayz, Alastair Martin, Gabriel Acevedo-Bolton, Michael T. Lawton, Randall Higashida, Wade S. Smith, William L. Young, and David Saloner. Phase-contrast magnetic resonance imaging measurements in intracranial aneurysms in vivo of flow patterns, velocity fields, and wall shear stress: Comparison with computational fluid dynamics. Magnetic Resonance in Medicine, 61(2):409-417, 2009.

[12] Shohei Miyazaki, Keiichi Itatani, Toyoki Furusawa, Teruyasu Nishino, Masataka Sugiyama, Yasuo Takehara, and Satoshi Yasukochi. Validation of numerical simulation methods in aortic arch using 4D Flow MRI. Heart and Vessels, 32(8):1032-1044, 2017. 
[13] Umberto Morbiducci, Raffaele Ponzini, Diego Gallo, Cristina Bignardi, and Giovanna Rizzo. Inflow boundary conditions for image-based computational hemodynamics: Impact of idealized versus measured velocity profiles in the human aorta. Journal of Biomechanics, 46(1):102-109, 2013.

[14] Kerem Pekkan, Diane De Zélicourt, Liang Ge, Fotis Sotiropoulos, David Frakes, Mark A. Fogel, and Ajit P. Yoganathan. Physics-driven CFD modeling of complex anatomical cardiovascular flows - A TCPC case study. Annals of Biomedical Engineering, 33(3):284-300, 2005.

[15] P. van Ooij, A. Guédon, C. Poelma, J. Schneiders, M. C.M. Rutten, H. A. Marquering, C. B. Majoie, E. van Bavel, and A. J. Nederveen. Complex flow patterns in a real-size intracranial aneurysm phantom: Phase contrast MRI compared with particle image velocimetry and computational fluid dynamics. NMR in Biomedicine, 25(1):14-26, 2012.

[16] Lewington S; Clarke R; Qizilbash N; Peto R; Collins R; Prospective Studies. Age-specific relevance of blood pressure to cause-specific mortality is best assessed by collaborative meta-analysis of individual participant data from the separate prospective studies. Lancet, 360:1903-1913, 2002.

[17] N. Sarwar, P. Gao, S. R. Kondapally Seshasai, and Et Al. Diabetes mellitus, fasting blood glucose concentration, and risk of vascular disease: A collaborative meta-analysis of 102 prospective studies. The Lancet, 375(9733):2215-2222, 2010.

[18] H. B. Hubert, M. Feinleib, P. M. McNamara, and W. P. Castelli. Obesity as an independent risk factor for cardiovascular disease: A 26-year follow-up of participants in the Framingham Heart Study. Circulation, 67(5):968-977, 1983.

[19] Caroline Cheng, Dennie Tempel, Rien Van Haperen, Arjen Van Der Baan, Frank Grosveld, Mat J.A.P. Daemen, Rob Krams, and Rini De Crom. 
Atherosclerotic lesion size and vulnerability are determined by patterns of fluid shear stress. Circulation, 113(23):2744-2753, 2006.

[20] Juan R. Cebral, Mariano Vazquez, Daniel M. Sforza, Guillaume Houzeaux, Satoshi Tateshima, Esteban Scrivano, Carlos Bleise, Pedro Lylyk, and Christopher M. Putman. Analysis of hemodynamics and wall mechanics at sites of cerebral aneurysm rupture. Journal of NeuroInterventional Surgery, 7(7):530-536, 2015.

[21] Pouya Youssefi, Rajan Sharma, C. Alberto Figueroa, and Marjan Jahangiri. Functional assessment of thoracic aortic aneurysms - The future of risk prediction? British Medical Bulletin, 121(1):61-71, 2017.

[22] Ryo Torii, Maria Kalantzi, Stergios Theodoropoulos, Padmini Sarathchandra, Xiao Yun $\mathrm{Xu}$, and Magdi H. Yacoub. Predicting impending rupture of the ascending aorta with bicuspid aortic valve: Spatiotemporal flow and wall shear stress. JACC: Cardiovascular Imaging, 6(9):1017-1019, 2013.

[23] Friso M. Rijnberg, Mark G. Hazekamp, Jolanda J. Wentzel, Patrick J.H. De Koning, Jos J.M. Westenberg, Monique R.M. Jongbloed, Nico A. Blom, and Arno A.W. Roest. Energetics of blood flow in cardiovascular disease: Concept and clinical implications of adverse energetics in patients with a fontan circulation. Circulation, 137(22):2393-2407, 2018.

[24] Riccardo Toninato, Jacob Salmon, Francesca Maria Susin, Andrea Ducci, and Gaetano Burriesci. Physiological vortices in the sinuses of Valsalva: An in vitro approach for bio-prosthetic valves. Journal of Biomechanics, 49(13):2635-2643, 2016.

[25] Yoram Richter and Elazer R. Edelman. Cardiology is flow. Circulation, 113(23):2679-2682, 2006.

[26] Petter Dyverfeldt, Malenka Bissell, Alex J. Barker, Ann F. Bolger, Carl Johan Carlhäll, Tino Ebbers, Christopher J. Francios, Alex Frydrychowicz, Ju- 
lia Geiger, Daniel Giese, Michael D. Hope, Philip J. Kilner, Sebastian Kozerke, Saul Myerson, Stefan Neubauer, Oliver Wieben, and Michael Markl. 4D flow cardiovascular magnetic resonance consensus statement. Journal of Cardiovascular Magnetic Resonance, 17(1):1-19, 2015.

[27] P. J. Kilner, G. Z. Yang, R. H. Mohiaddin, D. N. Firmin, and D. B. Longmore. Helical and retrograde secondary flow patterns in the aortic arch studied by three-directional magnetic resonance velocity mapping. Circulation, 88(5 I):2235-2247, 1993.

[28] Michael D Hope, Alison K Meadows, Thomas A Hope, Karen G Ordovas, David Saloner, Gautham P Reddy, Marcus T Alley, and Charles B Higgins. Clinical evaluation of aortic coarctation with $4 \mathrm{~d}$ flow $\mathrm{mr}$ imaging. Journal of Magnetic Resonance Imaging: An Official Journal of the International Society for Magnetic Resonance in Medicine, 31(3):711-718, 2010.

[29] Pim van Ooij, Wouter V. Potters, Jeremy Collins, Maria Carr, James Carr, S. Chris Malaisrie, Paul W.M. Fedak, Patrick M. McCarthy, Michael Markl, and Alex J. Barker. Characterization of Abnormal Wall Shear Stress Using 4D Flow MRI in Human Bicuspid Aortopathy. Annals of Biomedical Engineering, 43(6):1385-1397, 2015.

[30] Jelena Bock, Alex Frydrychowicz, Ramona Lorenz, Daniel Hirtler, Alex J. Barker, Kevin M. Johnson, Raoul Arnold, Hans Burkhardt, Juergen Hennig, and Michael Markl. In vivo noninvasive 4D pressure difference mapping in the human aorta: Phantom comparison and application in healthy volunteers and patients. Magnetic Resonance in Medicine, 66(4):1079-1088, 2011.

[31] T. Ebbers, L. Wigström, A. F. Bolger, B. Wranne, and M. Karlsson. Noninvasive measurement of time-varying three-dimensional relative pressure fields within the human heart. Journal of Biomechanical Engineering, 124(3):288293, 2002. 
[32] Joachim Lotz, Christian Meier, Andreas Leppert, and Michael Galanski. Cardiovascular flow measurement with phase-contrast MR imaging: Basic facts and implementation. Radiographics, 22(3):651-671, 2002.

[33] Andreas Harloff, Andrea Nußbaumer, Simon Bauer, Aurélien F. Stalder, Alex Frydrychowicz, Cornelius Weiller, Jürgen Hennig, and Michael Markl. In vivo assessment of wall shear stress in the atherosclerotic aorta using flowsensitive 4D MRI. Magnetic Resonance in Medicine, 63(6):1529-1536, 2010.

[34] Jan Bogaert, Steven Dymarkowski, Andrew M Taylor, and Vivek Muthurangu. Clinical cardiac MRI. Springer Science \& Business Media, 2012.

[35] Michael Markl, Andreas Harloff, Thorsten A. Bley, Maxim Zaitsev, Bernd Jung, Ernst Weigang, Mathias Langer, Jürgen Hennig, and Alex Frydrychowicz. Time-resolved 3D MR velocity mapping at 3T: Improved navigatorgated assessment of vascular anatomy and blood flow. Journal of Magnetic Resonance Imaging, 25(4):824-831, 2007.

[36] Penelope J Allisy-Roberts and Jerry Williams. Farr's physics for medical imaging. Elsevier Health Sciences, 2007.

[37] Ramkumar Krishnamurthy, Benjamin Cheong, and Raja Muthupillai. Tools for cardiovascular magnetic resonance imaging. Cardiovascular diagnosis and therapy, 4(2):104, 2014.

[38] Peter D Gatehouse and Lindsey A Crowe. Applications of phase-contrast flow and velocity imaging in cardiovascular MRI. European radiology, 15(10):2172-2184, 2005.

[39] Michael Markl, Alex Frydrychowicz, Sebastian Kozerke, Mike Hope, and Oliver Wieben. 4D flow MRI. Journal of Magnetic Resonance Imaging, 36(5):1015-1036, 2012. 
[40] Andreas Stadlbauer, Wilma van der Riet, Gerard Crelier, and Erich Salomonowitz. Accelerated time-resolved three-dimensional MR velocity mapping of blood flow patterns in the aorta using SENSE and k-t BLAST. European Journal of Radiology, 75(1):e15-e21, 2010.

[41] J. S. Milner, J. A. Moore, B. K. Rutt, and D. A. Steinman. Hemodynamics of human carotid artery bifurcations: Computational studies with models reconstructed from magnetic resonance imaging of normal subjects. Journal of Vascular Surgery, 28(1):143-156, 1998.

[42] Gary H Bernstein, Mat A and Zhou, Xiaohong Joe and Polzin, Jason A and King, Kevin F and Ganin, Alexander and Pelc, Norbert $\mathrm{J}$ and Glover. Concomitant gradient terms in phase contrast MR: analysis and correction. Magnetic resonance in medicine, 39(2):300-308, 1998.

[43] Johannes M. Peeters, Clemens Bos, and Chris J.G. Bakker. Analysis and correction of gradient nonlinearity and B0 inhomogeneity related scaling errors in two-dimensional phase contrast flow measurements. Magnetic Resonance in Medicine, 53(1):126-133, 2005.

[44] Mikhail V. Milchenko, Oleg S. Pianykh, and John M. Tyler. The fast automatic algorithm for correction of MR bias field. Journal of Magnetic Resonance Imaging, 24(4):891-900, 2006.

[45] Thomas R. McCauley, Constantino S. Pena, Christy K. Holland, Thomas B. Price, and John C. Gore. Validation of volume flow measurements with cine phase-contrast MR imaging for peripheral arterial waveforms. Journal of Magnetic Resonance Imaging, 5(6):663-668, 1995.

[46] Sergio Uribe, Philipp Beerbaum, Thomas Sangild Sørensen, Allan Rasmusson, Reza Razavi, and Tobias Schaeffter. Four-dimensional (4D) flow of the whole heart and great vessels using real-time respiratory self-gating. Magnetic Resonance in Medicine, 62(4):984-992, 2009. 
[47] Laura A. Freeman, Phillip M. Young, Thomas A. Foley, Eric E. Williamson, Charles J. Bruce, and Kevin L. Greason. CT and MRI assessment of the aortic root and ascending aorta. American Journal of Roentgenology, 200(6), 2013.

[48] A. Harloff, F. Albrecht, J. Spreer, A. F. Stalder, J. Bock, A. Frydrychowicz, J. Schollhorn, A. Hetzel, M. Schumacher, J. Hennig, and M. Markl. 3D blood flow characteristics in the carotid artery bifurcation assessed by flowsensitive 4D MRI at 3T. Magnetic Resonance in Medicine, 61(1):65-74, 2009.

[49] Susanne Schnell, Can Wu, and Sameer A Ansari. 4D MRI flow examinations in cerebral and extracerebral vessels. Ready for clinical routine? HHS Public Access. Curr Opin Neurol, 29(4):419-428, 2016.

[50] U Mberto M Morbiducci, R Affaele P Onzini, G Iovanna R Izzo, M Arcello C Adioli, A Ntonio E Sposito, F Rancesco D E C Obelli, A Lessandro D E L M Aschio, and F Ranco M Aria M Ontevecchi. In Vivo Quantification of Helical Blood Flow in Human Aorta by Time-Resolved Three-Dimensional Cine Phase Contrast Magnetic Resonance Imaging. Annals of biomedical engineering, 37(3):516-531, 2009.

[51] Michael Markl, Wolf Wallis, and Andreas Harloff. Reproducibility of flow and wall shear stress analysis using flow-sensitive four-dimensional MRI. Journal of Magnetic Resonance Imaging, 33(4):988-994, 2011.

[52] Thomas A. Hope, Michael Markl, Lars Wigström, Marcus T. Alley, D. Craig Miller, and Robert J. Herfkens. Comparison of flow patterns in ascending aortic aneurysms and volunteers using four-dimensional magnetic resonance velocity mapping. Journal of Magnetic Resonance Imaging, 26(6):14711479, 2007.

[53] Julio Garcia, Alex J. Barker, and Michael Markl. The Role of Imaging of 
Flow Patterns by 4D Flow MRI in Aortic Stenosis. JACC: Cardiovascular Imaging, 12(2):252-266, 2019.

[54] J.B. Landau, L.D, Sykes. Course of Theoretical Physics: Fluid Mechanics, 2nd edition (1987). Butterworth-Heinemann Ltd (1987), 1987.

[55] M. H. Zawawi, A. Saleha, A. Salwa, N. H. Hassan, N. M. Zahari, M. Z. Ramli, and Z. C. Muda. A review: Fundamentals of computational fluid dynamics (CFD). AIP Conference Proceedings, 2030(November), 2018.

[56] F. P. Glor, J. J M Westenberg, J. Vierendeels, M. Danilouchkine, and P. Verdonck. Validation of the coupling of magnetic resonance imaging velocity measurements with computational fluid dynamics in a U bend. Artificial Organs, 26(7):622-635, 2002.

[57] Jiyuan Tu, Kiao Inthavong, and Kelvin Kian Loong Wong. Computational Hemodynamics-Theory, Modelling and Applications. Springer, 2015.

[58] Charles G. Speziale. Turbulence modeling for time-dependent RANS and VLES: A review. AIAA Journal, 36(2):173-184, 1998.

[59] Adel M. Malek, Seth L. Alper, and Seigo Izumo. Hemodynamic shear stress and its role in atherosclerosis. Journal of the American Medical Association, 282(21):2035-2042, 1999.

[60] M. Shojima. Magnitude and Role of Wall Shear Stress on Cerebral Aneurysm. Computational Fluid Dynamic Study of 20 Middle Cerebral Artery Aneurysms. Stroke, 2004.

[61] C. Canstein, P. Cachot, A. Faust, A. F. Stalder, J. Bock, A. Frydrychowicz, J. Küffer, J. Hennig, and Michael Markl. 3D MR flow analysis in realistic rapid-prototyping model systems of the thoracic aorta: Comparison with in vivo data and computational fluid dynamics in identical vessel geometries. Magnetic Resonance in Medicine, 59(3):535-546, 2008. 
[62] Armin Leuprecht, Sebastian Kozerke, Peter Boesiger, and Karl Perktold. Blood flow in the human ascending aorta: A combined MRI and CFD study. Journal of Engineering Mathematics, 47(3-4):387-404, 2003.

[63] Merih Cibis, Wouter V Potters, Frank J H Gijsen, Henk Marquering, Antonius F W Van Der Steen, Aart J Nederveen, and Jolanda J Wentzel. Wall shear stress calculations based on 3D cine phase contrast MRI and computational fluid dynamics : a comparison study in healthy carotid arteries. NMR in Biomedicine, 27(7):826-834, 2014.

[64] Q Long, X Y Xu, B Ariff, S A Thom, A D Hughes, and A V Stanton. Reconstruction of blood flow patterns in a human carotid bifurcation: a combined CFD and MRI study. J Magn Reson Imaging, 11(3):299-311., 2000.

[65] John F LaDisa, Lars E Olson, Ismail Guler, Douglas a Hettrick, Said H Audi, Judy R Kersten, David C Warltier, and Paul S Pagel. Stent design properties and deployment ratio influence indexes of wall shear stress: a three-dimensional computational fluid dynamics investigation within a normal artery. Journal of applied physiology (Bethesda, Md. : 1985), 97(1):424430; discussion 416, 2004.

[66] Ajit P. Yoganathan, K. B. Chandran, and Fotis Sotiropoulos. Flow in prosthetic heart valves: State-of-the-art and future directions. Annals of Biomedical Engineering, 33(12 SPEC. ISS.):1689-1694, 2005.

[67] Laura Dempere-Marco, Estanislao Oubel, Marcelo Castro, Christopher Putman, Alejandro Frangi, and Juan Cebral. CFD analysis incorporating the influence of wall motion: Application to intracranial aneurysms. Lecture Notes in Computer Science (including subseries Lecture Notes in Artificial Intelligence and Lecture Notes in Bioinformatics), 4191 LNCS:438-445, 2006.

[68] B. Berthier, R. Bouzerar, and C. Legallais. Blood flow patterns in an anatomically realistic coronary vessel: Influence of three different reconstruction methods. Journal of Biomechanics, 35(10):1347-1356, 2002. 
[69] Ali Sarrami-Foroushani, Mohsen Nasr Esfahany, Abbas Nasiraei Moghaddam, Hamidreza Saligheh Rad, Kavous Firouznia, Madjid Shakiba, Hossein Ghanaati, Iain David Wilkinson, and Alejandro Federico Frangi. Velocity measurement in carotid artery: Quantitative comparison of time-resolved 3D phase-contrast MRI and image-based computational fluid dynamics. Iranian Journal of Radiology, 12(4):1-7, 2015.

[70] Jonathan B. Thomas, Jaques S. Milner, Brian K. Rutt, and David A. Steinman. Reproducibility of image-based computational fluid dynamics models of the human carotid bifurcation. Annals of Biomedical Engineering, 31(2):132-141, 2003.

[71] DN Weston, SJ and Wood, NB and Tabor, G and Gosman, AD and Firmin. Combined MRI and CFD Analysis of Fully Developed Steady and Pulsatile Laminar Flow through a Bend. Journal of Magnetic Resonance Imaging, 8(5):1158-1171, 1998.

[72] Nigel B. Wood, E. Mimech, Simon J. Weston, Philip J. Kilner, A. David Gosman, and David N. Firmin. Combined MR imaging and CFD simulation of flow in the human descending aorta. Journal of Magnetic Resonance Imaging, 13(5):699-713, 2001.

[73] Ji Young Moon, Dae Chul Suh, Yong Sang Lee, Young Woo Kim, and Joon Sang Lee. Considerations of blood properties, outlet boundary conditions and energy loss approaches in computational fluid dynamics modeling. Neurointervention, 9(1):1-8, 2014.

[74] Paul D. Morris, Andrew Narracott, Hendrik Von Tengg-Kobligk, Daniel Alejandro Silva Soto, Sarah Hsiao, Angela Lungu, Paul Evans, Neil W. Bressloff, Patricia V. Lawford, D. Rodney Hose, and Julian P. Gunn. Computational fluid dynamics modelling in cardiovascular medicine. Heart, 102(1):18-28, 2016. 
[75] G. E. Elsinga, F. Scarano, B. Wieneke, and B. W. Van Oudheusden. Tomographic particle image velocimetry. Experiments in Fluids, 41(6):933-947, 2006.

[76] F. Scarano. Tomographic PIV: Principles and practice. Measurement Science and Technology, 24(1), 2013.

[77] Markus Raffel, Christian E Willert, Fulvio Scarano, Christian J Kähler, Steve T Wereley, and Jürgen Kompenhans. Particle image velocimetry: a practical guide. Springer, 2018.

[78] Nicolas A. BUCHMANN, Miharu YAMAMOTO, Mark JERMY, and Tim DAVID. Particle Image Velocimetry (PIV) and Computational Fluid Dynamics (CFD) Modelling of Carotid Artery Haemodynamics under Steady Flow: A Validation Study. Journal of Biomechanical Science and Engineering, 5(4):421-436, 2010.

[79] Matthew D. Ford, Sang Wook Lee, Stephen P. Lownie, David W. Holdsworth, and David A. Steinman. On the effect of parent-aneurysm angle on flow patterns in basilar tip aneurysms: Towards a surrogate geometric marker of intra-aneurismal hemodynamics. Journal of Biomechanics, 41(2):241-248, 2008.

[80] Marcelo Raschi, Fernando Mut, Greg Byrne, Christopher M. Putman, Satoshi Tateshima, Fernando Viñuela, Tetsuya Tanoue, Kazuo Tanishita, and Juan R. Cebral. CFD and PIV analysis of hemodynamics in a growing intracranial aneurysm. International Journal for Numerical Methods in Biomedical Engineering, 28(2):214-228Raschi, M., Mut, F., Byrne, G., Putman, C., 2012.

[81] Carine Guivier-Curien, Valérie Deplano, and Eric Bertrand. Validation of a numerical 3-D fluid-structure interaction model for a prosthetic valve based on experimental PIV measurements. Medical Engineering and Physics, 31(8):986-993, 2009. 
[82] Anna Maria Tango, Jacob Salmonsmith, Andrea Ducci, and Gaetano Burriesci. Validation and Extension of a Fluid-Structure Interaction Model of the Healthy Aortic Valve. Cardiovascular Engineering and Technology, 9(4):739-751, 2018.

[83] Pierre Bouillot, Olivier Brina, Rafik Ouared, Hasan Yilmaz, Karl Olof Lovblad, Mohamed Farhat, and Vitor Mendes Pereira. Computational fluid dynamics with stents: Quantitative comparison with particle image velocimetry for three commercial off the shelf intracranial stents. Journal of NeuroInterventional Surgery, 8(3):309-315, 2016.

[84] Rafael Medero, Carson Hoffman, and Alejandro Roldán-Alzate. Comparison of 4D Flow MRI and Particle Image Velocimetry Using an In Vitro Carotid Bifurcation Model. Annals of Biomedical Engineering, 46(12):2112-2122, 2018.

[85] Helmut Baumgartner, Volkmar Falk, Jeroen J. Bax, Michele De Bonis, Christian Hamm, Per Johan Holm, Bernard Iung, Patrizio Lancellotti, Emmanuel Lansac, Daniel Rodriguez Muñoz, Raphael Rosenhek, Johan Sjögren, Pilar Tornos Mas, Alec Vahanian, Thomas Walther, Olaf Wendler, Stephan Windecker, Jose Luis Zamorano, Marco Roffi, Ottavio Alfieri, Stefan Agewall, Anders Ahlsson, Emanuele Barbato, Hector Bueno, Jean Philippe Collet, Ioan Mircea Coman, Martin Czerny, Victoria Delgado, Donna Fitzsimons, Thierry Folliguet, Oliver Gaemperli, Gilbert Habib, Wolfgang Harringer, Michael Haude, Gerhard Hindricks, Hugo A. Katus, Juhani Knuuti, Philippe Kolh, Christophe Leclercq, Theresa A. McDonagh, Massimo Francesco Piepoli, Luc A. Pierard, Piotr Ponikowski, Giuseppe M.C. Rosano, Frank Ruschitzka, Evgeny Shlyakhto, Iain A. Simpson, Miguel Sousa-Uva, Janina Stepinska, Giuseppe Tarantini, Didier Tche, Victor Aboyans, Hovhannes K. Kzhdryan, Julia Mascherbauer, Fuad Samadov, Vadim Shumavets, Guy Van Camp, Daniela Loncar, Daniel Lovric, Georgios M. Georgiou, Katerina Linhartova, Nikolaj Ihlemann, Magdy Abdelhamid, 
Teele Pern, Anu Turpeinen, Elizabeta Srbinovska-Kostovska, Ariel Cohen, Zviad Bakhutashvili, Huseyin Ince, Manolis Vavuranakis, Andras Temesvari, Thorarinn Gudnason, Darren Mylotte, Rafael Kuperstein, Ciro Indolfi, Yury Pya, Gani Bajraktari, Alina Kerimkulova, Ainars Rudzitis, Vaida Mizariene, Frederic Lebrun, Daniela Cassar Demarco, Latifa Oukerraj, Berto J. Bouma, Terje Kristian Steigen, Monika Komar, Luisa Maria De Moura Branco, Bogdan A. Popescu, Vladimir Uspenskiy, Marina Foscoli, Ljiljana Jovovic, Iveta Simkova, Matjaz Bunc, Jose Antonio Vazquez de Prada, Martin Stagmo, Beat Andreas Kaufmann, Abdallah Mahdhaoui, Engin Bozkurt, Elena Nesukay, and Stephen J.D. Brecker. 2017 ESC/EACTS Guidelines for the management of valvular heart disease. European Heart Journal, 38(36):2739_ 2786, 2017.

[86] Carole A. Warnes, Roberta G. Williams, Thomas M. Bashore, John S. Child, Heidi M. Connolly, Joseph A. Dearani, Pedro del Nido, James W. Fasules, Thomas P. Graham, Ziyad M. Hijazi, Sharon A. Hunt, Mary Etta King, Michael J. Landzberg, Pamela D. Miner, Martha J. Radford, Edward P. Walsh, and Gary D. Webb. ACC/AHA 2008 Guidelines for the Management of Adults with Congenital Heart Disease: a report of the American College of Cardiology/American Heart Association Task Force on Practice Guidelines (writing committee to develop guidelines on the management of a. Circulation, 118(23):714-833, 2008.

[87] Tino Ebbers and Gunnar Farnebäck. Improving computation of cardiovascular relative pressure fields from velocity MRI. Journal of Magnetic Resonance Imaging, 30(1):54-61, 2009.

[88] Sebastian B.S. Krittian, Pablo Lamata, Christian Michler, David A. Nordsletten, Jelena Bock, Chris P. Bradley, Alex Pitcher, Philip J. Kilner, Michael Markl, and Nic P. Smith. A finite-element approach to the direct computation of relative cardiovascular pressure from time-resolved MR velocity data. Medical Image Analysis, 16(5):1029-1037, 2012. 
[89] Cristóbal Bertoglio, Rodolfo Nuñez, Felipe Galarce, David Nordsletten, and Axel Osses. Relative pressure estimation from velocity measurements in blood flows: State-of-the-art and new approaches. International Journal for Numerical Methods in Biomedical Engineering, 34(2):1-16, 2018.

[90] David Marlevi, Bram Ruijsink, Maximilian Balmus, Desmond DillonMurphy, Daniel Fovargue, Kuberan Pushparajah, Cristóbal Bertoglio, Massimiliano Colarieti-Tosti, Matilda Larsson, Pablo Lamata, C. Alberto Figueroa, Reza Razavi, and David A. Nordsletten. Estimation of Cardiovascular Relative Pressure Using Virtual Work-Energy. Scientific Reports, 9(1):1-16, 2019.

[91] Joseph AC SZMYD, Janusz S and SUZUKI, Kenjiro and KOLENDA, Zygmunt Sz and HUMPHREY. A study of thermo-fluid phenomena with uncertainties by making use of interactive computational-experimental methodology. JSME international journal. Ser. 2, Fluids engineering, heat transfer, power, combustion, thermophysical properties, 35(4):599-607, 1992.

[92] Dale Barker, Xiang Yu Huang, Zhiquan Liu, Tom Auligné, Xin Zhang, Steven Rugg, Raji Ajjaji, Al Bourgeois, John Bray, Yongsh Eng Chen, Meral Demirtas, Yong Run Guo, Tom Henderson, Wei Huang, Hui Chuan Lin, John Michalakes, Syed Rizvi, and Xiaoyan Zhang. The weather research and forecasting model's community variational/ensemble data assimilation system: WRFDA. Bulletin of the American Meteorological Society, 93(6):831-843, 2012.

[93] T Hayase and S Hayashi. State Estimator of Flow as an Integrated Computational Method With Feedback of Online Experimental Measurement. Journal of Fluids Engineering, 119(December 1997):814-822, 1997.

[94] Kenichi Funamoto, Toshiyuki Hayase, Yoshifumi Saijo, and Tomoyuki Yambe. Numerical experiment for ultrasonic-measurement-integrated sim- 
ulation of three-dimensional unsteady blood flow. Annals of Biomedical Engineering, 36(8):1383-1397, 2008.

[95] Kenichi Funamoto, Yoshitsugu Suzuki, Toshiyuki Hayase, Takashi Kosugi, and Haruo Isoda. Numerical validation of MR-measurement-integrated simulation of blood flow in a cerebral aneurysm. Annals of Biomedical Engineering, 37(6):1105-1116, 2009.

[96] Vinicius C. Rispoli, Jon F. Nielsen, Krishna S. Nayak, and Joao L. A. Carvalho. Computational fluid dynamics simulations of blood flow regularized by 3D phase contrast MRI. BioMedical Engineering OnLine, 14(1):110, 2015.

[97] Jelena Bock, Alex Frydrychowicz, Aurélien F. Stalder, Thorsten A. Bley, Hans Burkhardt, Jürgen Hennig, and Michael Markl. 4D phase contrast MRI at 3 T: Effect of standard and blood-pool contrast agents on SNR, PC-MRA, and blood flow visualization. Magnetic Resonance in Medicine, 63(2):330 $338,2010$.

[98] Glycerine Producers' Association et al. Physical properties of glycerine and its solutions. Glycerine Producers’ Association, 1963.

[99] A. Sciacchitano. Uncertainty quantification in particle image velocimetry. Measurement Science and Technology, 30(9), 2019.

[100] Joseph M. Sherwood, David Holmes, Efstathios Kaliviotis, and Stavroula Balabani. Spatial distributions of red blood cells significantly alter local haemodynamics. PLoS ONE, 9(6), 2014.

[101] Anders Nilsson, Karin Markenroth Bloch, Marcus Carlsson, Einar Heiberg, and Freddy Ståhlberg. Variable velocity encoding in a three-dimensional, three-directional phase contrast sequence: Evaluation in phantom and volunteers. Journal of Magnetic Resonance Imaging, 36(6):1450-1459, 2012. 
[102] K. Sudo, M. Sumida, and H. Hibara. Experimental investigation on turbulent flow in a circular-sectioned 180-degree bend. Experiments in Fluids, 28(1):51-57, 2000.

[103] R. Trip, D. J. Kuik, J. Westerweel, and C. Poelma. An experimental study of transitional pulsatile pipe flow. Physics of Fluids, 24(1), 2012.

[104] J. Peacock, T. Jones, C. Tock, and R. Lutz. The onset of turbulence in physiological pulsatile flow in a straight tube. Experiments in Fluids, 24(1):1-9, 1998.

[105] Athanasia Kalpakli Vester, Ramis Örlü, and P. Henrik Alfredsson. Turbulent Flows in Curved Pipes: Recent Advances in Experiments and Simulations. Applied Mechanics Reviews, 68(5):050802, 2016.

[106] Lun Shin Yao and Stanley A. Berger. Flow in heated curved pipes. Journal of Fluid Mechanics, 88(2):339-354, 1978.

[107] Christian J. Kähler, Sven Scharnowski, and Christian Cierpka. On the uncertainty of digital PIV and PTV near walls. Experiments in Fluids, 52(6):16411656, 2012.

[108] Liam Morris, Patrick Delassus, Pierce Grace, Fintin Wallis, Michael Walsh, and Tim McGloughlin. Effects of flat, parabolic and realistic steady flow inlet profiles on idealised and realistic stent graft fits through Abdominal Aortic Aneurysms (AAA). Medical Engineering and Physics, 28(1 SPEC. ISS.):19-26, 2006.

[109] Senol Piskin and M. Serdar Celebi. Analysis of the effects of different pulsatile inlet profiles on the hemodynamical properties of blood flow in patient specific carotid artery with stenosis. Computers in Biology and Medicine, 43(6):717-728, 2013.

[110] Alifer D. Bordones, Matthew Leroux, Vitaly O. Kheyfets, Yu An Wu, Chia Yuan Chen, and Ender A. Finol. Computational Fluid Dynamics Model- 
ing of the Human Pulmonary Arteries with Experimental Validation. Annals of Biomedical Engineering, 46(9):1309-1324, 2018.

[111] Raimund Erbel, Victor Aboyans, Catherine Boileau, Eduardo Bossone, Roberto Di Bartolomeo, Holger Eggebrecht, Arturo Evangelista, Volkmar Falk, Herbert Frank, Oliver Gaemperli, Martin Grabenwöger, Axel Haverich, Bernard Iung, Athanasios John Manolis, Folkert Meijboom, Christoph A. Nienaber, Marco Roffi, Hervé Rousseau, Udo Sechtem, Per Anton Sirnes, Regula S. Von Allmen, Christiaan J.M. Vrints, Jose Luis Zamorano, Stephan Achenbach, Helmut Baumgartner, Jeroen J. Bax, Héctor Bueno, Veronica Dean, Christi Deaton, Çetin Erol, Robert Fagard, Roberto Ferrari, David Hasdai, Arno Hoes, Paulus Kirchhof, Juhani Knuuti, Philippe Kolh, Patrizio Lancellotti, Ales Linhart, Petros Nihoyannopoulos, Massimo F. Piepoli, Piotr Ponikowski, Juan Luis Tamargo, Michal Tendera, Adam Torbicki, William Wijns, Stephan Windecker, Martin Czerny, John Deanfield, Carlo Di Mario, Mauro Pepi, Maria Jesus Salvador Taboada, Marc R. Van Sambeek, and Charalambos Vlachopoulos. 2014 ESC guidelines on the diagnosis and treatment of aortic diseases. European Heart Journal, 35(41):2873-2926, 2014.

[112] Kristopher S. Cunningham and Avrum I. Gotlieb. The role of shear stress in the pathogenesis of atherosclerosis. Laboratory Investigation, 85(1):9-23, 2005.

[113] Armin Leuprecht, Karl Perktold, Sebastian Kozerke, and Peter Boesiger. Combined CFD and MRI study of blood flow in a human ascending aorta model. Biorheology, 39(3-4):425-429, 2002.

[114] Alex Frydrychowicz, Aurélien F. Stalder, Maximilian F. Russe, Jelena Bock, Simon Bauer, Andreas Harloff, Alexander Berger, Mathias Langer, Jürgen Hennig, and Michael Markl. Three-dimensional analysis of segmental wall shear stress in the aorta by flow-sensitive four-dimensional-MRI. Journal of Magnetic Resonance Imaging, 30(1):77-84, 2009. 
[115] Fraser M. Callaghan and Stuart M. Grieve. Spatial resolution and velocity field improvement of 4D-flow MRI. Magnetic Resonance in Medicine, 78(5):1959-1968, 2017.

[116] C. A. Kousera, N. B. Wood, W. A. Seed, R. Torii, D. O’Regan, and X. Y. Xu. A numerical study of aortic flow stability and comparison with in vivo flow measurements. Journal of Biomechanical Engineering, 135(1), 2013.

[117] Julius M. Gardin, Cora S. Burn, William J. Childs, and Walter L. Henry. Evaluation of blood flow velocity in the ascending aorta and main pulmonary artery of normal subjects by Doppler echocardiography. American Heart Journal, 107(2):310-319, 1984.

[118] Alex Frydrychowicz, Aurélien F. Stalder, Maximilian F. Russe, Jelena Bock, Simon Bauer, Andreas Harloff, Alexander Berger, Mathias Langer, Jürgen Hennig, and Michael Markl. Three-dimensional analysis of segmental wall shear stress in the aorta by flow-sensitive four-dimensional-MRI. Journal of Magnetic Resonance Imaging, 30(1):77-84, 2009.

[119] Philippe Reymond, Paolo Crosetto, Simone Deparis, Alfio Quarteroni, and Nikos Stergiopulos. Physiological simulation of blood flow in the aorta: Comparison of hemodynamic indices as predicted by 3-D FSI, 3-D rigid wall and 1-D models. Medical Engineering and Physics, 35(6):784-791, 2013.

[120] Fraser M. Callaghan and Stuart M. Grieve. Translational Physiology: Normal patterns of thoracic aortic wall shear stress measured using four-dimensional flow MRI in a large population. American Journal of Physiology - Heart and Circulatory Physiology, 315(5):H1174-H1181, 2018.

[121] Pim Van Ooij, Wouter V. Potters, Aart J. Nederveen, Bradley D. Allen, Jeremy Collins, James Carr, S. Chris Malaisrie, Michael Markl, and Alex J. Barker. A methodology to detect abnormal relative wall shear stress on the full surface of the thoracic aorta using four-dimensional flow MRI. Magnetic Resonance in Medicine, 73(3):1216-1227, 2015. 
[122] F Stalder, Alex Frydrychowicz, Max F Russe, Kuncheng Li, Jan G Korvink, and Michael Markl. Assessment of Flow Instabilities in the Healthy Aorta Using Flow-Sensitive MRI. \{Journal of Magnetic Resonance Imaging, 33(4):839-846, 2011.

[123] Jonas Lantz, Tino Ebbers, Jan Engvall, and Matts Karlsson. Numerical and experimental assessment of turbulent kinetic energy in an aortic coarctation. Journal of Biomechanics, 46(11):1851-1858, 2013.

[124] A. D. Caballero and S. Laín. Numerical simulation of non-Newtonian blood flow dynamics in human thoracic aorta. Computer Methods in Biomechanics and Biomedical Engineering, 18(11):1200-1216, 2015.

[125] Xiao Liu, Yubo Fan, Xiaoyan Deng, and Fan Zhan. Effect of non-Newtonian and pulsatile blood flow on mass transport in the human aorta. Journal of Biomechanics, 44(6):1123-1131, 2011.

[126] Jonas Lantz, Johan Renner, and Matts Karlsson. Wall shear stress in a subject specific human aorta - Influence of fluid-structure interaction. International Journal of Applied Mechanics, 3(4):759-778, 2011.

[127] A. D. Caballero and S. Laín. A Review on Computational Fluid Dynamics Modelling in Human Thoracic Aorta. Cardiovascular Engineering and Technology, 4(2):103-130, 2013.

[128] W. A. Seed and N. B. Wood. Velocity patterns in the aorta. Cardiovascular Research, 5(3):319-330, 1971.

[129] R. M. Nerem, J. A. Rumberger, D. R. Gross, R. L. Hamlin, and G. L. Geiger. Hot film anemometer velocity measurements of arterial blood flow in horses. Circulation Research, 34(2):193-203, 1974.

[130] Umberto Morbiducci, Raffaele Ponzini, Giovanna Rizzo, Marcello Cadioli, Antonio Esposito, Franco Maria Montevecchi, and Alberto Redaelli. Mechanistic insight into the physiological relevance of helical blood flow in the 
human aorta: An in vivo study. Biomechanics and Modeling in Mechanobiology, 10(3):339-355, 2011.

[131] A. Kamiya and T. Togawa. Adaptive regulation of wall shear stress to flow change in the canine carotid artery. American Journal of Physiology - Heart and Circulatory Physiology, 8(1):14-21, 1980.

[132] C. G. Caro, J. M. Fitz-Gerald, and R. C. Schroter. Atheroma and arterial wall shear. Observation, correlation and proposal of a shear dependent mass transfer mechanism for atherogenesis. Proceedings of the Royal Society of London. Series B. Biological sciences, 177(46):109-159, 1971.

[133] Donald L. Fry. Certain chemorheologic considerations regarding the blood vascular interface with particular reference to coronary artery disease. Circulation, 40(5s4):IV-38, 1969.

[134] Veronique Peiffer, Spencer J. Sherwin, and Peter D. Weinberg. Does low and oscillatory wall shear stress correlate spatially with early atherosclerosis? A systematic review. Cardiovascular Research, 99(2):242-250, 2013.

[135] David N Ku. Blood flow in arteries. Annual review of fluid mechanics, 29(1):399-434, 1997.

[136] Jin Suo, John N. Oshinski, and Don P. Giddens. Blood flow patterns in the proximal human coronary arteries: Relationship to atherosclerotic plaque occurrence. MCB Molecular and Cellular Biomechanics, 5(1):9-18, 2008.

[137] Emanuele Cecchi, Cristina Giglioli, Serafina Valente, Chiara Lazzeri, Gian Franco Gensini, Rosanna Abbate, and Lucia Mannini. Role of hemodynamic shear stress in cardiovascular disease. Atherosclerosis, 214(2):249256, 2011.

[138] Christopher K Zarins, Don P Giddens, B K Bharadvaj, Vikrom S Sottiurai, Robert F Mabon, and Seymour Glagov. Carotid bifurcation atherosclerosis. 
Quantitative correlation of plaque localization with flow velocity profiles and wall shear stress. Circulation Research, 53(4):502-514, 1983.

[139] T. Asakura and T. Karino. Flow patterns and spatial distributions of atherosclerotic lesions in human coronary arteries. Circulation Research, 66(4):1045-1066, 1990.

[140] David De Wilde, Bram Trachet, Guido R.Y. De Meyer, and Patrick Segers. Shear Stress Metrics and Their Relation to Atherosclerosis: An In Vivo Follow-up Study in Atherosclerotic Mice. Annals of Biomedical Engineering, 44(8):2327-2338, 2016.

[141] G. Coppola and C. Caro. Oxygen mass transfer in a model three-dimensional artery. Journal of the Royal Society Interface, 5(26):1067-1075, 2008.

[142] Sven Petersson, Petter Dyverfeldt, and Tino Ebbers. Assessment of the accuracy of MRI wall shear stress estimation using numerical simulations. Journal of Magnetic Resonance Imaging, 36(1):128-138, 2012.

[143] Phillip E. Gates, Arati Gurung, Luciano Mazzaro, Kuni Aizawa, Salim Elyas, William D. Strain, Angela C. Shore, and Robin Shandas. Measurement of Wall Shear Stress Exerted by Flowing Blood in the Human Carotid Artery: Ultrasound Doppler Velocimetry and Echo Particle Image Velocimetry. Ultrasound in Medicine and Biology, 44(7):1392-1401, 2018.

[144] R. A. Garrick, U. S. Ryan, and F. P. Chinard. Water permeability of isolated endothelial cells at different temperatures. American Journal of Physiology Cell Physiology, 255(3), 1988.

[145] Mohamad G. Ghosn, Michael Leba, Astha Vijayananda, Panteha Rezaee, Joel D. Morrisett, and Kirill V. Larin. Effect of temperature on permeation of low-density lipoprotein particles through human carotid artery tissues. Journal of Biophotonics, 2(10):573-580, 2009. 
[146] L. F. Fajardo and S. D. Prionas. Endothelial cells and hyperthermia. International journal of hyperthermia, 10(3):347-353, 1994.

[147] Fatemeh Kabirian, Ghassem Amoabediny, Nooshin Haghighipour, Nasim Salehi-Nik, and Behrouz Zandieh-Doulabi. Nitric oxide secretion by endothelial cells in response to fluid shear stress, aspirin, and temperature. Journal of Biomedical Materials Research - Part A, 103(3):1231-1237, 2015.

[148] Yasutoshi Ishihara, Arturo Calderon, Hidehiro Watanabe, Kazuya Okamoto, Yoshinori Suzuki, Kagayaki Kuroda, and Yutaka Suzuki. A precise and fast temperature mapping using water proton chemical shift. Magnetic Resonance in Medicine, 34(6):814-823, 1995.

[149] Umberto Morbiducci, Annette M. Kok, Brenda R. Kwak, Peter H. Stone, David A. Steinman, and Jolanda J. Wentzel. Atherosclerosis at arterial bifurcations: Evidence for the role of haemodynamics and geometry. Thrombosis and Haemostasis, 115(3):484-492, 2016.

[150] D. N. Ku, D. P. Giddens, C. K. Zarins, and S. Glagov. Pulsatile flow and atherosclerosis in the human carotid bifurcation. Positive correlation between plaque location and low and oscillating shear stress. Arteriosclerosis, 5(3):293-302, 1985.

[151] Loic Boussel, Vitaliy Rayz, Charles McCulloch, Alastair Martin, Gabriel Acevedo-Bolton, Michael Lawton, Randall Higashida, Wade S. Smith, William L. Young, and David Saloner. Aneurysm growth occurs at region of low wall shear stress: Patient-specific correlation of hemodynamics and growth in a longitudinal study. Stroke, 39(11):2997-3002, 2008.

[152] Leonid Goubergrits, Eugenie Riesenkampff, Pavlo Yevtushenko, Jens Schaller, Ulrich Kertzscher, Anja Hennemuth, Felix Berger, Stephan Schubert, and Titus Kuehne. MRI-based computational fluid dynamics for diagnosis and treatment prediction: Clinical validation study in patients with 
coarctation of aorta. Journal of Magnetic Resonance Imaging, 41(4):909916, 2015.

[153] P. A. Stonebridge and C.M. Brophy. Spiral laminar flow in arteries? The Lancet, 338(8779):1360-1361, 1991.

[154] A Moffatt, HK and Tsinober. Helicity in laminar and turbulent flow. Annual review of fluid mechanics, 24(11):281-312, 1992. 